Published in final edited form as:

Physiol Rev. 2007 January ; 87(1): 315-424.

\title{
Nitric Oxide and Peroxynitrite in Health and Disease
}

\author{
PÁL PACHER, JOSEPH S. BECKMAN, and LUCAS LIAUDET \\ Section on Oxidative Stress Tissue Injury, Laboratory of Physiologic Studies, National Institutes of \\ Health, National Institute of Alcohol Abuse and Alcoholism, Bethesda, Maryland; Linus Pauling \\ Institute, Department of Biochemistry and Biophysics, 2011 Agricultural and Life Sciences, Oregon \\ State University, Corvallis, Oregon; and Department of Intensive Care Medicine, University Hospital, \\ Lausanne, Switzerland
}

\section{Abstract}

The discovery that mammalian cells have the ability to synthesize the free radical nitric oxide (NO) has stimulated an extraordinary impetus for scientific research in all the fields of biology and medicine. Since its early description as an endothelial-derived relaxing factor, NO has emerged as a fundamental signaling device regulating virtually every critical cellular function, as well as a potent mediator of cellular damage in a wide range of conditions. Recent evidence indicates that most of the cytotoxicity attributed to NO is rather due to peroxynitrite, produced from the diffusion-controlled reaction between $\mathrm{NO}$ and another free radical, the superoxide anion. Peroxynitrite interacts with lipids, DNA, and proteins via direct oxidative reactions or via indirect, radical-mediated mechanisms. These reactions trigger cellular responses ranging from subtle modulations of cell signaling to overwhelming oxidative injury, committing cells to necrosis or apoptosis. In vivo, peroxynitrite generation represents a crucial pathogenic mechanism in conditions such as stroke, myocardial infarction, chronic heart failure, diabetes, circulatory shock, chronic inflammatory diseases, cancer, and neurodegenerative disorders. Hence, novel pharmacological strategies aimed at removing peroxynitrite might represent powerful therapeutic tools in the future. Evidence supporting these novel roles of $\mathrm{NO}$ and peroxynitrite is presented in detail in this review.

\section{INTRODUCTION}

Nitric oxide (NO) is an omnipresent intercellular messenger in all vertebrates, modulating blood flow, thrombosis, and neural activity. The biological production of $\mathrm{NO}$ is also important for nonspecific host defense, but NO itself is unlikely directly to kill intracellular pathogens and tumors. Although NO is often described as highly toxic and reactive, it is not. Inhaling low concentrations of gaseous NO is approved by the Food and Drug Administration for the treatment of persistent pulmonary hypertension of the newborn $(53,411,412,593,680,681$, 1143). In addition, $\mathrm{NO}$ can be produced for 80 years by neurons in human brain without overt toxicity. Paradoxically, the production of the same molecule can become highly damaging to the same neurons within a few minutes during pathological challenges as occur after cerebral ischemia. How is this possible? The reaction of $\mathrm{NO}$ with superoxide $\left(\mathrm{O}_{2}{ }^{--}\right)$to form the much more powerful oxidant peroxynitrite $\left(\mathrm{ONOO}^{-}\right)$is a key element in resolving the contrasting roles of NO in physiology and pathology.

Neither superoxide nor NO is particularly toxic in vivo because there are efficient means to minimize their accumulation $(72,74)$. Superoxide is rapidly removed by high concentrations of scavenging enzymes called superoxide dismutases (SOD) with distinct isoenzymes located in the mitochondria, cytoplasm, and extracellular compartments. NO is rapidly removed by its

Address for reprint requests and other correspondence: P. Pacher, National Institutes of Health, NIAAA, Laboratory of Physiological Studies, 5625 Fishers Lane MSC 9413, Room 2N-17, Bethesda, MD 20892-9413 (e-mail: pacher@ mail.nih.gov). 
rapid diffusion through tissues into red blood cells $(161,639)$, where it is rapidly converted to nitrate by reaction with oxyhemoglobin (Fig. 1). This limits the biological half-life of NO in vivo to less than a second, whereas the concentrations of NO relevant for cellular signaling can persist in phosphate-buffered saline for an hour (79). However, when both superoxide and NO are synthesized within a few cell diameters of each other, they will combine spontaneously to form peroxynitrite by a diffusion-limited reaction (583). In essence, every time NO and superoxide collide, they form peroxynitrite. No enzyme is required to form peroxynitrite because no enzyme can possibly catalyze any reaction as fast. NO is the only known biological molecule that reacts faster with superoxide and is produced in high enough concentrations to outcompete endogenous levels of superoxide dismutase. Consequently, the kinetics and thermodynamics of the reaction of superoxide with $\mathrm{NO}$ make the formation of peroxynitrite inevitable in vivo.

Generally, the physiological literature is content with discussing NO as a physiological signaling agent without considering its complex and controversial chemistry. A lot of biology is masked in the fog of the nebulous description reactive nitrogen species. Many of the biological effects attributed to $\mathrm{NO}$ are in fact mediated by peroxynitrite. While requiring some knowledge of the underlying convoluted chemistry of free radicals and nitrogen oxides, recognition of these reactions in vivo will produce a deeper understanding of the underlying biology and help resolve many puzzling controversies. In this review, we describe the substantial progress made in understanding the biological chemistry of peroxynitrite and its many roles in virtually all disease processes affecting humans. New tools enabling the imaging of superoxide formation in vivo $(1446,1455)$ combined with the careful interpretation of nitration and the use of scavengers for peroxynitrite can allow these processes to be experimentally dissected $(370,371)$.

The formation of reactive nitrogen species is not an inescapable consequence of synthesizing NO. NO is efficiently removed by reacting with oxyhemoglobin to form nitrate, which prevents even the highest rates of NO synthesis from directly reacting with oxygen to form significant amounts of nitrogen dioxide. However, the simultaneous activation of superoxide synthesis along with NO will completely transform the biological actions of NO by forming peroxynitrite. Several enzyme complexes, such as NADPH oxidases (NADPHox) and xanthine oxidase, can be activated in many cellular systems to actively produce large amounts of superoxide. What happens when superoxide and NO are produced simultaneously in close proximity? Modestly increasing superoxide and NO each at a 10 -fold greater rate will increase peroxynitrite formation by 100 -fold. Under proinflammatory conditions, simultaneous production of superoxide and NO can be strongly activated to increase production 1,000-fold, which will increase the formation of peroxynitrite by a 1,000,000-fold (Fig. 2). Without superoxide, the formation of nitrogen dioxide by the reaction of $\mathrm{NO}$ with oxygen is miniscule by comparison. NO and superoxide do not even have to be produced within the same cell to form peroxynitrite, because NO can so readily move through membranes and between cells (Fig. 1).

Although peroxynitrite is a strong oxidant, it reacts at a relatively slow rate with most biological molecules. With its reaction with carbon dioxide and other major reactions in cells taken into account, peroxynitrite can still diffuse quite far on a cellular scale (Fig. 1). Peroxynitrite is able to traverse cell membranes in part through anion channels $(312,824)$. The contrast with hydroxyl radical is dramatic $(73,78)$. Hydroxyl radical is formed by a rather slow reaction via the reaction of ferrous iron with hydrogen peroxide, but is so reactive that it can only diffuse about the diameter of a typical protein (586). In contrast, peroxynitrite is formed each time superoxide and $\mathrm{NO}$ collide, but reacts slowly enough to react more selectively throughout the cell. That makes the biological and pathological implications of peroxynitrite far more interesting because it can have more subtle and specific actions on cells. The implications of 
biological oxidants having more specific actions in vivo have been described by Nathan in his essay on biological specificity of a third kind (933).

\section{NITRIC OXIDE}

\section{A. Discovery of Nitric Oxide as a Biological Molecule}

The regulation and synthesis of NO by mammalian cells has been the focus of many excellent reviews (935), as have many of its physiological and pathological actions $(595,890,893,895$, 1201). With around 80,000 references invoking NO listed in PubMed, it may be difficult to remember how controversial was the initial proposal that $\mathrm{NO}$ was a biological molecule. There were early indications that were ignored for many decades. Haldane and co-workers (56) reported in the 1920s of a man found dead of apparent carbon monoxide poisoning. However, analysis of the blood showed that the stable red color persisting in hemoglobin at autopsy was due to nitroso-hemoglobin rather than carboxy-hemoglobin (56). There was no evidence for the consumption of nitrite that might account for the nitroso-hemoglobin. Commoner and coworkers (1387) in the 1960s demonstrated the nitroso-heme signals could be detected by EPR spectroscopy. Tannenbaum's group (475), while investigating the role of nitrite and nitrosamines in food in the induction of carcinogenesis, characterized nitrite and nitrate metabolism in healthy student volunteers. Curiously, more nitrate was being secreted than could be accounted for by ingestion. When one of the students became ill with the flu, nitrate levels in urine increased enormously.

For a century, nitrovasodilators had been used clinically without understanding their mechanism of action. Alfred Nobel lamented the irony that he was taking nitroglycerin to treat angina after making his fortune developing dynamite. Increased levels of cGMP produced by guanylate cyclase within vascular smooth muscle were discovered to allow blood vessels to relax and thus increase blood flow (596). The endogenous agents responsible for activating guanylate cyclase remained mysterious, although nitrovasodilators were able to activate the enzyme. The enzyme contained a heme group that was essential for this activation. Murad's group (130) found that NO itself, as well as a variety of oxidants, was able to activate guanylate cyclase. However, the possibility that NO might be synthesized in mammals was considered to be too far fetched for another decade. The more likely candidates for activators of guanylate cyclase were considered to be nitroso thiols and possibility that NO, a major air pollutant, could be the endogenous regulator was generally dismissed.

Major progress came from the curiosity of Furchgott and Zawadzki (422) in understanding how a technician in his laboratory was able to isolate aortas that relaxed in vitro when exposed to acetylcholine. Acetylcholine was a well-known vasodilating agent when injected in vivo, but generally caused isolated blood vessels to constrict in vitro. Furchgott and Zawadzki (422) recognized that the difference was caused by mechanical damage to the vascular rings as they were being cut, which stripped the single layer of endothelial cells off the blood vessels. With the preparation of a spiral cut of blood vessels, the endothelium was preserved and responded by relaxing when acetylcholine was added. Furthermore, Furchgott and Zawadzki (422) established that acetylcholine-treated endothelium was releasing a diffusible factor that would relax endothelium-denuded blood vessels by activating guanylate cyclase. The diffusible factor, termed the endothelium-derived relaxing factor (EDRF), was quickly inactivated by oxyhemoglobin and was inherently unstable in the perfusion cascades used to study the vasorelaxation. EDRF had a half-life of only 6-8 s in Krebs-Henseleit buffer saturated with $100 \%$ oxygen and $30-60 \mathrm{~s}$ in the same buffer saturated with room air (21\% oxygen). Addition of low concentrations of SOD to the perfusion cascade doubled the half-life of EDRF in Krebs-Henseleit buffer, while agents known to generate superoxide diminished its activity $(484,894,1089)$. Consequently, the sensitivity to superoxide and oxyhemoglobin became standard criteria to verify the production of EDRF in isolated cells. Moreover, EDRF was 
proposed to be a protective factor by scavenging superoxide. In 1986, Furchgott proposed that NO was the elusive EDRF produced by endothelium, with Ignarro providing additional evidence supporting the identification $(594,596,597)$. Moncada's group (892), adapting a gasphase chemiluminescent detector used for monitoring NO pollution in the atmosphere, was able to directly measure NO produced in vivo.

The identification of NO as EDRF was greatly facilitated by the independent work of John Hibbs, who was investigating how macrophages kill cancer cells. Hibbs found that different batches of fetal calf serum had widely varying effects on the tumoricidal activity of macrophages in vivo. He observed in the local slaughterhouse that calf serum at the time was prepared from blood collected in buckets and deduced that endotoxin from contaminating bacteria was responsible for activating the macrophages. He also noticed that activated macrophages rapidly depleted the media of some nutrient necessary for their tumoricidal activity $(542,543)$. By supplementing the depleted media with each component, he discovered that arginine was the major compound that could restore the tumoricidal activity of macrophages. He also showed that the macrophages were producing nitrite and nitrate by oxidizing arginine to citrulline, but did not make the connection that macrophages may be producing NO and peroxynitrite. The production of NO by macrophages was established by Stuehr and Nathan (1208) and peroxynitrite by Ischiropoulos et al. (611). He deduced that arginine derivatives might be useful inhibitors and discovered that methylarginine blocked the tumoricidal activity of macrophages. Thus he provided the background for Moncada's group to show that EDRF was synthesized from arginine and that methylarginine blocked its biological activity.

A major difficulty in the identification of EDRF is the fact that endothelium produces relatively small amounts and is not an abundant cell in vivo. But the identification of EDRF as NO made sense of many puzzling results in the brain, where cGMP was known to be involved in many intracellular functions. Deguchi and Yoshioka (305) painstakingly purified a low-molecularweight compound from nervous tissue that was required to activate soluble guanylate cyclase and showed that it was unexpectedly arginine. For many years, it had been recognized that the brain had all of the enzymes necessary to convert citrulline to arginine, but curiously lacked arginase that is necessary for the complete urea cycle to be functional. Although the urea cycle was not functional in the brain, genetic deficiencies in these enzymes often resulted in phenotypes with neurological defects. Garthwaite and coworkers $(433,435)$ connected the dots and showed that under normal physiological conditions the brain produced $\sim 20$ times more NO than the entire vasculature. Since NO synthase produces citrulline from arginine, the presence of the remaining urea cycle enzymes was rationalized. Later, he identified ODQ, $1 \mathrm{H}-$ $(1,2,4)$ oxadiazolo(4,3-a)quinoxalin-1-one, as a potent inhibitor that prevented the activation of guanylate cyclase by NO (436), which has proven to be a crucial tool for unraveling the physiological action of $\mathrm{NO}$ in vitro.

The brain proved to be a rich source of NO synthesis and allowed the first NO synthase (NOS) to be cloned and purified (133-135). It is called nNOS (neuronal NOS) or NOS1 as it was the first synthase to be cloned. Eventually, it was realized that the histological stain for NADPH diaphorase in brain corresponded to the distribution of nNOS $(292,568)$. The NOS1 gene has the most complex genomic organization in humans with multiple splice variants being produced $(468,1164)$. The first knock-out mouse still retained substantial NOS1 activity in muscle and some neuronal populations because of alternative splicing that bypassed the second exon that had been targeted for deletion $(574,1394)$. Consequently, several studies finding no protection in the knockout of NOS1 do not necessarily prove the lack of involvement of NO (375). 
The second NOS to be cloned was isolated from macrophages and known as NOS2 or iNOS (inducible NOS) because it is readily induced in many tissues by proinflammatory cytokines. Unlike the neuronal or endothelial NOS isoenzymes, NOS2 is not regulated by changes in intracellular calcium. However, careful examination of purified preparations of iNOS revealed that calmodulin was so tightly bound that it copurified with iNOS $(215,1395)$. Consequently, the enzyme appeared to be unresponsive to physiologically relevant changes in calcium concentrations. Because NOS2 can be strongly induced by proinflammatory stimuli, it is often called a high-output source of NO. However, the enzyme does not produce NO at a substantially greater rate measured for neuronal or endothelial NOS, just more of the protein can transiently be induced and normal calcium levels are sufficient to fully activate NOS2 $(934,935)$.

The first source of NO identified, endothelial NO synthase was the last to be cloned and is known as eNOS or NOS3. NOS3 binds to plasma membranes and is typically associated with caveolin (387). It is strongly activated by the entry of calcium through membrane-bound receptors and is also regulated by phosphorylation (1122). NOS3 is also found in neurons and other tissues in addition to endothelium.

\section{B. The Selective Reactivity of Nitric Oxide}

To understand why NO itself is not highly reactive, it is instructive to remember that $\mathrm{NO}$ is an intermediate between molecular oxygen $\left(\mathrm{O}_{2}\right)$ and nitrogen $\left(\mathrm{N}_{2}\right)$ (Fig. 3). All three have low solubility and readily diffuse through membranes as easily as through cytoplasm. While molecular oxygen is thermodynamically a strong oxidant, it has a limited kinetic reactivity as evidenced by life being possible in an atmosphere of $21 \%$ oxygen without spontaneous combustion. Molecular oxygen has two unpaired electrons in separate orbitals, which prevents this biradical from directly reacting with most biological molecules. However, the unpaired electrons on molecular oxygen allow it to bind strongly with metals such as the iron in hemoglobin and in cytochrome-c oxidase. Molecular oxygen will also react quickly with the unpaired electron on other free radicals, which leaves the second unpaired electron available for further reactions: $\mathrm{R} \bullet+\bullet \mathrm{OO} \bullet \rightarrow \mathrm{R}-\mathrm{OO} \bullet \rightarrow$ further reactions.

Hence, molecular oxygen propagates free radical damage and has a central role in oxidative stress.

In contrast, nitrogen gas $\left(\mathrm{N}_{2}\right)$ is one of the most inert molecules known. Because $\mathrm{NO}$ is essentially a hybrid between molecular nitrogen and oxygen, $\mathrm{NO}$ is generally less reactive than molecular oxygen. In particular, NO has only one unpaired electron, which allows it to bind strongly to the iron in heme groups, which is crucial to its biological activity of activating guanylate cyclase and slowing mitochondrial respiration by binding to cytochrome- $c$ oxidase. Like molecular oxygen, $\mathrm{NO}$ also reacts with free radicals quickly, but $\mathrm{NO}$ is chain terminating. For example, NO can convert thiyl radicals into nitrosothiols by acting as a chain-terminating agent: RS• $+\bullet \mathrm{NO} \rightarrow$ RS-NO.

The chain-terminating reactions account for many of the antioxidant properties attributed to $\mathrm{NO}$, but this is an oversimplification. NO does not directly repair radical damage as does ascorbate, tocopherol, or glutathione, but rather forms transient intermediate products that have distinct biological activities. These intermediate products can often be repaired by antioxidants to regenerate the original compound. Depending on one's point of view, nitrosative stress can also be viewed as an antioxidant activity.

\section{Diffusion and Signaling Properties of Nitric Oxide}

NO is often considered to be just another signaling molecule. But it is important to consider how NO communicates information to understand why NO has so many physiological roles 
in vivo. The production of cGMP by guanylate cyclase is the major signal transduction mechanism of NO. Soluble guanylate cyclase contains the same heme protoporphyrin IX as hemoglobin with iron in the ferrous form that binds NO with great affinity. Deoxyhemoglobin binds NO with a 10,000-fold greater affinity than molecular oxygen $(1151,1286)$. Only $5-10$ $\mathrm{nM} N \mathrm{NO}$ is necessary to activate guanylate cyclase. NO can diffuse from where it is synthesized into surrounding cells where it will activate soluble guanylate cyclase in the target tissue to produce cGMP. In turn, cGMP activates cGMP-dependent kinases in the target tissue that modulates intracellular calcium levels to modulate many diverse activities in the target tissues.

However, the unique properties of NO confer an important distinction that allows NO to be used for local signaling in virtually every organ system. For many signaling molecules, receptors in target tissues can distinguish two closely related molecules like norepinephrine or epinephrine because the appropriate receptors can recognize subtle variations in shape. With only two atoms, NO cannot be readily distinguished by its shape. Thus information is reflected by changes in its local concentration. The longer NO is present, the greater the amount of cGMP that will be formed. The system depends on NO being constantly removed or else guanylate cyclase will remain fully activated.

The rapid infusion of NO has major implications for how information is communicated. NO is a small hydrophobic molecule that crosses cell membranes without channels or receptors as readily as molecular oxygen and carbon dioxide. The diffusion coefficient of NO in water at $37^{\circ} \mathrm{C}$ is slightly faster than oxygen and carbon dioxide (1377), which is ideal for quickly transmitting information over short distances. Because NO is freely permeable to membranes, NO will repeatedly diffuse in as well as out of a cell over the time span of a second. The average molecular velocity of a molecule with the mass of $\mathrm{NO}$ is $\sim 400 \mathrm{~m} / \mathrm{s}$ at room temperature. The trajectory of NO in solution is repeatedly changed by making $\sim 10$ billion collisions each second. Consequently, the path of a NO molecule will follow long but highly convoluted trails that can repeatedly cross cell membranes during its half-life of $\sim 1 \mathrm{~s}$. A single molecule of NO can readily move between cells many times within this time span.

The hydrophobicity of NO will allow slightly faster diffusion in a lipid membrane than in water so that membranes provide effectively no barrier to NO. Hence, diffusion away from a single cell producing NO contributes far more substantially to the loss of NO than do the reactions of NO within the cell (728). When a group of cells simultaneously produces NO, the concentration of $\mathrm{NO}$ within a single cell will be much greater than if that cell was producing $\mathrm{NO}$ alone. Most of the $\mathrm{NO}$ one breathes when stuck in a traffic jam results from the surrounding cars rather than the car itself. An important consequence is that $\mathrm{NO}$ can integrate and average the activity of the local group of cells (728).

Red blood cells provide a drain for NO that creates a sharp diffusion gradient leading to the vasculature $(161,1294,1385)$. The addition of a red blood cell outside of a cell will capture much of the NO produced inside of this cell, because the hemoglobin will greatly reduce the reentry of NO into the cell (728). For example, endothelium can make 10- to 40-fold more NO than needed to activate guanylate cyclase as measured by microelectrodes placed against individual isolated cells. However, red blood cells will be major sinks in vivo, so the majority of the NO will be quickly lost to the vascular compartment $(196,1293)$. In fact, the packaging of hemoglobin into red blood cells is important to limit the rate of scavenging of NO. Lysis of red blood cells causes vasoconstriction by more efficient scavenging of $\mathrm{NO}$ and is a major limitation of giving hemoglobin-based blood substitutes. Even with hemoglobin packaged into red blood cells, the production of $100 \mathrm{nM} \mathrm{NO}$ by endothelium would be necessary to achieve 5-10 nM concentrations in smooth muscle containing guanylate cyclase, because such a large fraction is consumed by red blood cells. NO that has diffused into the smooth muscle can also diffuse back down the diffusion gradient to the red blood cell (Fig. 1). In the perfusion cascades 
used to originally assay EDRF, the excess production of NO in the absence of hemoglobin allowed the dilation of endothelium-denuded artery rings far removed down the perfusion cascade.

Although the biological half-life of NO is only a scant few seconds in vivo, a second is long compared with a simple neural reflex or to the time needed to contract a muscle. A sprinter can run $\sim 10 \mathrm{~m}$ within the reported half-life of $\mathrm{NO}$ (far shorter than the total distance covered of a molecule of $\mathrm{NO}$ as it bounces around inside and between cells in the same time). The relatively short overall distance that $\mathrm{NO}$ can diffuse limits its action to only a few cells near the source of production. Thus NO produced in the gut, for example, will not influence its actions in the central nervous system (CNS). On the other hand, the intermediate lifetime of NO coupled with its rapid diffusion through most tissues allows NO to integrate and modulate complex physiological processes (1293).

In effect, $\mathrm{NO}$ is the equivalent of a shock absorber that dampens the oscillations of a car driving over a bumpy road. By affecting levels of calcium in target tissues through the actions of cGMP, NO can modulate the extent that target tissues respond to stress. When a distal vascular bed dilates to supply more blood flow to an actively working muscle, the upstream blood vessels must also dilate to support the increased blood flow. Exceeding the Reynolds number results in turbulent flow, increasing the local stress on blood vessels (479). The stress of turbulent flow is counterpoised by myogenic contraction that would cause a further increase in shear stress in the absence of endothelial production of NO. Left unopposed, further muscle contraction amplifies turbulent flow that in the extreme will cause a catastrophic collapse of blood flow to distal vascular beds. However, turbulence induces endothelium to synthesize NO via shear-induced stress. The resulting local relaxation of the underlying vascular smooth muscle increases the diameter of the blood vessel to restore laminar flow and thereby ensures a laminar distribution of blood between vessels (479). With prolonged turbulence, additional mechanisms are activated that can lead to the formation of superoxide and the pathogenic conversion of NO to peroxynitrite as described below.

NO can convey information by more subtle means than just simply its local concentration. The life span of NO is relatively long compared with the firing of nerve or even the activation of a neural network, and thus can help to integrate neuronal activity in small volumes of the brain (1385). Gally et al. (426) proposed that NO provides an important signal-averaging mechanism to control synaptic plasticity in the brain. The spatial organization of the nervous system is determined by the temporally correlated activation of neurons. A general principle controlling the organization of the developing brain is that groups of neurons activated in synchrony tend to project to similar regions, whereas neurons with uncorrelated activity will project into different regions (897). NO appears to be one determinant of this neuronal localization because it is ideally suited to carry information about neuronal activity in a retrograde manner, opposite to the normal mode of an activated neuron passing information down its axon and releasing a neurotransmitter across the synaptic junction. NO produced by dendrites will diffuse radially to surrounding synapses and will not distinguish between synapses in direct contact with that particular dendrite versus those that are localized in the same region $(434,1385,1391)$.

The nNOS (NOS1) is particularly well suited to produce NO in a manner that facilitates synaptic plasticity $(336,1188)$. The amino terminus of NOS1 contains an additional sequence lacking on NOS2 and NOS3 that anchors the enzyme to the cytoskeleton in postsynaptic boutons beneath the $N$-methyl-D-aspartate (NMDA) receptor (547). The NMDA receptor has been implicated in learning and development as well as in many forms of excitotoxic neurodegeneration (149) (see sect. VI, $F$ and $G$ ). The NMDA receptor only activates when a neuron has been partially depolarized, as occurs when a neuron has been firing frequently. In addition, the receptor must bind glutamate and glycine to the extracellular surface for the 
channel to open. A local group of neurons that are firing repeatedly for a few milliseconds is sufficient to cause local increase in NO. The synthesis of NO is initiated by extracellular calcium entering the neuron through the NMDA receptor. NO plays an important role in longterm potentiation, the most widely studied neuronal equivalent of learning in vitro (126, 1137).

Local neuronal activity can be temporally integrated because NO will only be synthesized by localized groups of neurons that had been depolarized through repeated activation that is necessary to open NMDA receptors (426). NO can help enhance the synaptic efficiency of surrounding axonal arbors of neurons that have been active, whereas the axonal arbors from neurons that have not been activated will be weakened. The ability to modulate local groups of neurons on a moderate time scale is one in the reasons why the CNS is a major source of NOS and why it undergoes radical changes in expression throughout development $(154,462$, 647,1188).

\section{Cytotoxic Effects of Nitric Oxide}

Although NO is reported to have many potentially toxic effects, many of them are more likely mediated by its oxidation products rather than NO itself. Thus NO does not directly attack DNA, as was initially believed, but this effect instead depends on its conversion into higher nitrogen oxides (1375). It also does not directly cause the ribosylation of glyceraldehyde-3phosphate dehydrogenase (323), but rather reacts with a sulfhydryl anion in the active site of the enzyme (888). Furthermore, the early consideration that NO produced by activated macrophages would inactivate the iron/sulfur centers in tumor cell mitochondria $(329,542)$ has been reevaluated by a series of experiments indicating that the NO-dependent inactivation of iron/sulfur centers was in fact mediated by peroxynitrite $(182,523)$. Indeed, activated macrophages produce both $\mathrm{NO}$ and superoxide, so the inactivation of mitochondria in tumor cells could well have been mediated by peroxynitrite (1062).

NO may reversibly inhibit enzymes with transition metals or with free radical intermediates in their catalytic cycle. NO in micromolar concentrations will reversibly inhibit catalase and cytochrome $P$-450 $(153,1376)$. It also can inhibit ribonucleotide reductase, the enzyme responsible for DNA synthesis that contains a tyrosine radical. Subsequent inhibition of DNA may inhibit viral replication. However, the inhibition of ribonucleotide reductase is rapidly reversible and lost when NO is less than a few micromolar in concentration. Large continuous fluxes of NO are necessary to keep ribonucleotide reductase inhibited, which would occur only under major inflammatory conditions or in the neighborhood of an activated macrophage. Enormous amounts of oxygen are required to maintain synthesis of $\mathrm{NO}$ in micromolar concentrations. Since it takes two oxygens per NO produced, and if the half-life of NO in vivo was as long as $7 \mathrm{~s}$, then $120 \mathrm{nmol} \mathrm{O}_{2}$ would be needed per gram tissue per minute to maintain $\mathrm{NO}$ at a steady-state concentration of $1 \mu \mathrm{M}$ ! NO in the submicromolar range can also reversibly inhibit cytochrome- $c$ oxidase $(141,220,1161)$, which may transiently increase the leakage of superoxide from the electron transport chain. The superoxide so formed could then react with NO to generate peroxynitrite, which would cause irreversible injury to the mitochondria.

\section{E. Superoxide and the Hydroxyl Radical Theory of Radical Damage}

The recognition that superoxide could be a biologically significant molecule was at one time viewed as unlikely as NO. Many thought superoxide was chemically far too reactive to be produced in vivo. Inorganic chemists produce superoxide by burning molten potassium metal with pure molecular oxygen. As a solid, potassium superoxide $\left(\mathrm{KO}_{2}{ }^{\bullet}\right)$ is a powerful oxidant that reacts vigorously when added to water. Originally, chemists wrote the structure as $\mathrm{K}_{2} \mathrm{O}_{4}$. In 1934, Pauling and Neuman (1013) deduced from quantum mechanics that $2 \mathrm{KO}_{2}$ • 
would be more stable than a molecule with four oxygen atoms and proposed the name superoxide for this one-electron reduced state of molecular oxygen.

In the mid 1960s, Fridovich (410) investigated the oxygen-dependent reduction of cytochrome $c$ by the flavin-containing xanthine oxidase. Oxygen would be expected to oxidize cytochrome $c$ rather than to facilitate reduction. A protein was found in blood cells that would inhibit the reduction of cytochrome $c$, which was purified by McCord and Fridovich in 1969 (859). This protein had previously been purified as a copper binding protein, but McCord and Fridovich (688) showed this protein was an efficient scavenger of superoxide. Fridovich's group soon isolated a manganese-containing version from bacteria and also present in mitochondria (1026). The ability of SOD to scavenge superoxide provided strong evidence that superoxide was a biologically relevant molecule. This provided strong impetus to the theory that the univalent reduction of molecular oxygen to produce the free radicals superoxide, hydrogen peroxide, and hydroxyl radical were responsible for the toxicity of oxygen in biological systems. Oxygen has been known since the 1950s to greatly amplify the toxicity resulting from radiation by amplifying radical damage.

While the name superoxide implies that superoxide should be a powerful oxidant, superoxide more generally behaves as a mild reductant under physiological conditions rather than a "super"-oxidizing agent. This is because superoxide exists naturally as a small anion $\left(\mathrm{O}_{2}{ }^{--}\right)$, which is more likely to surrender its electron than to accept a second electron from another biological molecule. Hence, the reduction potential for superoxide is about $-0.1 \mathrm{~V}$ at physiological oxygen concentrations. Superoxide is a strong oxidant when it is protein mated, but its $\mathrm{p} K_{\mathrm{a}}$ is $\sim 4.3$ so that it will directly oxidize positively charged chemical moieties such as iron/sulfur centers. The destruction of iron/sulfur centers in mitochondria has been well described in SOD knockout mice $(761,910)$. Aconitase is another iron/sulfur protein that is particularly susceptible to inactivation by superoxide (523).

The limited chemical reactivity of superoxide created considerable controversy about the role of superoxide in cellular toxicity (1125). To explain how a more potent oxidant might be generated by superoxide, the iron-catalyzed formation of hydroxyl radical from hydrogen peroxide was proposed. Because hydroxyl radical was well known to be a potent oxidant and important in radiation-induced damage to biological molecules, it became widely accepted as the major toxin produced in vivo. While widely described in many pathology textbooks, there are many limitations to the hydroxyl radical theory. In particular, the formation of hydroxyl radical requires the presence of an appropriately chelated iron atom reacting first with superoxide to become reduced and then with hydrogen peroxide to form hydroxyl radical. Scavengers of either superoxide or hydrogen peroxide such as SOD can effectively inhibit this reaction. The reaction of iron with hydrogen peroxide tends to have rather slow rate constants. Finally, hydroxyl radical is so reactive that it will react with virtually every biological molecule within a very short diffusion distance. Hydroxyl radical will diffuse on average less than the diameter of the typical protein. Thus the biological relevance of hydroxyl radical is limited because it is formed by a slow reaction while being so highly reactive that its toxicity becomes limited by reacting with too many irrelevant biological targets.

Still administration of SOD to experimental animals subjected to ischemia and other inflammatory stresses could provide substantial benefits. At the same time that McCord and Fridovich had purified SOD, another group purified an anti-inflammatory compound from cow blood that turned out to be identical to SOD (578). By the mid 1980s, 6,000,000 doses of SOD had been administered as an anti-inflammatory agent to patients in Europe. These studies clearly implicated superoxide as having a major role in promoting tissue pathology. However, the biological targets of superoxide remained obscure. The protection of EDRF by SOD suggested that it might be a major target of superoxide in vivo. When EDRF was identified as 
NO, it raised the possibility that its product, peroxynitrite, could be a significant and unrecognized oxidant in vivo.

\section{PEROXYNITRITE}

\section{A. Historical Perspectives}

At the time when NO was discovered, the controversy about the reactivity of superoxide and its significance to pathology was at its nadir. A few publications concerning peroxynitrite were scattered in the older chemical literature or found in studies of atmospheric pollution. The diffusion-limited reaction of $\mathrm{NO}$ with $\mathrm{HO}_{2} \bullet$ was recognized to be the major source of nitrogen dioxide and hydroxyl radical in the atmosphere and strongly implicated in the formation of smog.

The historical perspective of the discovery of peroxynitrite has been nicely reviewed by Koppenol (708). As early as 1901, the unusual oxidizing power of acidified nitrite mixed with hydrogen peroxide was noted. As an oxidant, peroxynitrite attracted little attention because it produced a bewildering array of products even with fairly simple starting substrates such as phenol. In 1970, several investigators more thoroughly characterized the chemistry of peroxynitrite showing that it decomposed to form hydroxyl radical and nitrogen dioxide (85, 401,580,581,832). Hughes and Nicklin (580) established the most commonly used extinction coefficient $\left(1.67 \mathrm{mM}^{-1} \cdot \mathrm{cm}^{-1}\right)$ through indirect measurements. Later, Bohle et al. (108) prepared pure peroxynitrite and determined the extinction coefficient as $1.70 \mathrm{mM}^{-1} \cdot \mathrm{cm}^{-1}$.

Peroxynitrite can also be formed through the ultraviolet radiation of solid crystals of nitrate, turning clear crystals yellow (678). This is potentially more than a laboratory curiosity. The attempts by the Viking missions to Mars in 1976 to detect life may have been confronted by ultraviolet-induced peroxynitrite formation in nitrate found in the Martian soil (1031).

Peroxynitrite may also prove useful to solubilize chromium III by oxidizing it to chromium VI from the radioactive sludge found in the Hanford nuclear storage facilities in Washington state (808). Solid chromium III causes glass to weaken more quickly and requires more glass to be used for vitrification of radioactive waste. Treatment with peroxynitrite can reduce the amount of glass needed to entrapp trans-uranium elements by a factor of two, which has huge economic benefits to reduce the cost of permanent storage of radioactive waste.

In studies of the fate of $\mathrm{NO}$ in the ocean, Zafirou and co-workers $(104,1428)$ showed that superoxide reacts with $\mathrm{NO}$ to form peroxynitrite. The most commonly cited rate constant for this reaction was measured as $6.7 \times 10^{9} \mathrm{M}^{-1} \cdot \mathrm{s}^{-1}$ (583). Koppenol et al. (937) found using three different flash photolysis methods that the rate was slightly faster $\left(16-20 \times 10^{9} \mathrm{M}^{-1}\right.$. $\mathrm{s}^{-1}$ ), while others report slightly slower rate constants as determined by pulse radiolysis (3-4 $\times 10^{9} \mathrm{M}^{-1} \cdot \mathrm{s}^{-1}$ ). The rate of superoxide reacting with SOD is $\sim 2 \times 10^{9} \mathrm{M}^{-1} \cdot \mathrm{s}^{-1}$. Hence, NO is the one molecule produced in high enough concentrations and reacts fast enough to outcompete endogenous SOD for superoxide. As described below, the interactions of superoxide, NO, and peroxynitrite with SOD are more complex than this simple analysis indicates.

\section{B. Biological Chemistry}

In 1990, the first papers suggesting that peroxynitrite could be a biological oxidant were published $(71,75)$. At the time, much of the literature suggested that $\mathrm{NO}$ was a scavenger of superoxide and thus acting as an antioxidant $(379,855)$. However, Beckman et al. (75) showed peroxynitrite was a far more effective means of producing hydroxyl radical than the widely accepted reaction of reduced iron with hydrogen peroxide (known as the Fenton reaction or the iron-catalyzed Haber-Weiss reaction). These results were confirmed by Hogg et al. using systems to cogenerate superoxide and NO $(287,559)$. In addition, peroxynitrite produced 
nitrogen dioxide, which could lead to novel oxidation products that were previously only suspected to occur after exposure to cigarette smoke or to air pollution.

Peroxynitrite itself is also a strong oxidant and can react directly with electron-rich groups, such as sulfhydryls (1056), iron-sulfur centers (182), zinc-thiolates (245), and the active site sulfhydryl in tyrosine phosphatases (1254). Curiously, the reaction rate constants are relatively slow for these second-order reactions, ranging from $10^{3}$ to $10^{6} \mathrm{M}^{-1} \cdot \mathrm{s}^{-1}$. Peroxynitrite is surprisingly stable in solution, considering its strong oxidizing potential and that it is $36 \mathrm{kcal} /$ mol higher in energy than its isomer nitrate. The unusual stability of peroxynitrite results in part because it folds into a stable cis-conformation where the negative charge is localized over the entire molecule (1291). The molecules are further stabilized by forming strong hydrogen bonds with two or three waters (1290). The limited reactivity of peroxynitrite with most molecules makes it unusually selective as an oxidant, which increases its influence over biological processes.

Although peroxynitrite is a strong oxidant, the anion $\left(\mathrm{ONOO}^{-}\right)$also reacts directly with nucleophiles, molecules with a partial positive charge. One example of major importance is carbon dioxide. The carbon is surrounded by two oxygens, which effectively pull electron density away. Hence, carbon dioxide reacts with hydroxyl anion to form bicarbonate. In the same way, carbon dioxide reacts with peroxynitrite to form a transient intermediate nitrosoperoxycarbonate that rapidly decomposes homolytically to nitrogen dioxide and carbonate radical. Because carbon dioxide is nearly $1 \mathrm{mM}$ in cells $(\sim 10,000$ times greater than hydrogen ions), the formation of carbonate radicals is more likely to occur in vivo than the formation of hydroxyl radical per se from HOONO (peroxynitrous acid, the conjugated acid of peroxynitrite). Carbonate radical is more selective than hydroxyl radical but will initiate many of the damaging reactions commonly attributed to hydroxyl radical in the biological literature and is perhaps more significant as a biological oxidant (873).

Multiple oxidative pathways can form both hydroxyl and carbonate radical independently of peroxynitrite or NO. However, peroxynitrite can also produce novel products such as nitrotyrosine, nitrotryptophan, and nitrated lipids that serve as important biological markers in vivo. Ohshima et al. (967) developed a mass spectrometric method to measure nitrotyrosine of smokers, but was surprised to find significant amounts in the urine of non-smoking humans as well. At the same time, Ischiropoulos and co-workers found that peroxynitrite caused bovine SOD to turn yellow, which was eventually traced to the nitration of the sole tyrosine at position $108(609,612,1174)$.

A major limitation to the acceptance of peroxynitrite or any reactive nitrogen species as a significant player in disease was whether enough could be produced to be damaging. Significant questions were raised as to whether human "macrophages" produced NO and whether its synthesis was mostly a rodent-specific phenomenon. However, in subsequent studies, it became clear that the regulation of inducible NOS was under tighter control in humans $(936,1196,1353)$.

The development of antibodies that recognize nitrotyrosine also provided a major impetus to the study of peroxynitrite (80). Nitrotyrosine can easily be detected in Formalin-fixed tissue, and the antibodies are not species specific. This allowed the antibodies to be broadly applicable $(1327,1414)$. Nitrotyrosine was first shown to be localized in human atherosclerotic lesions and vascular muscle (80). Later, it was found in a huge number of most disease-affected tissues (see Tables 4 and 5 and sect. VI) and its presence confirmed by mass spectrometric analyses $(244,1098,1397)$. One of the major values of nitrotyrosine is that it provided strong evidence that the production of $\mathrm{NO}$ was sufficient to produce observable products in virtually every human disease (606). 
In recent years, it has become common to use nitrotyrosine as a marker of nitrosative stress, although this is a serious misrepresentation of the underlying processes. Incubating large amounts of $\mathrm{NO}(1-2 \mathrm{mM})$ aerobically with proteins, tissue homogenates, or cell cultures does not yield significant amounts of nitrotyrosine (80). However, peroxynitrite readily yields nitrotyrosine in yields of 3-14\%. Although a high concentration of NO forms nitrogen dioxide, the concentration of nitrogen dioxide is thousands of times more dilute than NO. Consequently, the nitrogen dioxide reacts rapidly with the $\mathrm{NO}$ to form $\mathrm{N}_{2} \mathrm{O}_{3}$, which is a nitrosating agent that is far more likely to react with thiols as well as primary and secondary amines than with tyrosine. Nitrotyrosine readily decomposes to tyrosine and nitrite. Hence, nitrosative stress produces products such as nitrosothiols and nitrosamines, but nitrotyrosine and nitrotryptophan are more stable products and indicative of a more intense oxidative stress. This stress is better characterized as nitrative stress.

Nitrotyrosine is extremely useful for measuring the formation of peroxynitrite, but this requires additional experimental validation. The first evidence for peroxynitrite formation came from SOD as a catalyst of tyrosine nitration to detect peroxynitrite being formed from freshly isolated rat alveolar macrophages (611). As isolated, these macrophages produced NO, but not superoxide. No nitration of a tyrosine analog could be observed under these basal conditions. When superoxide synthesis by NADPH oxidase was activated with a phorbol ester, nitration could be visually observed by the tyrosine analog turning a faint yellow color. The amount of nitration could be increased paradoxically by adding SOD (Fig. 4). SOD catalyzes tyrosine nitration by peroxynitrite, but would generally be expected to reduce peroxynitrite formation by scavenging superoxide. To parse these conflicting actions, SOD was chemically treated by a combination of two well-established methods to reduce its superoxide scavenging activity by $>99 \%$. The copper remained bound to the SOD after these treatments, and the ability to catalyze tyrosine nitration was unaffected. Unexpectedly, unmodified SOD was equally effective at catalyzing nitration as the inactivated SOD, suggesting that nitration was enhanced by metal-dependent catalysis (copper) at the active site of the enzyme. This also argued that NO was reacting so quickly with superoxide produced on the cell surface by NADPH oxidase that SOD added to the extracellular media could not effectively remove superoxide under these identical conditions. What was particularly important about this study was that oxygen consumption, NO production, superoxide formation, and nitration were all quantified as well as the effects of NOS inhibition on these quantities. When superoxide formation was stimulated to a threefold greater rate than NO synthesis, all of these data showed that $\mathrm{NO}$ was being quantitatively converted to peroxynitrite.

The production of oxidants is a crucial mechanism for neutrophils and macrophages to damage or kill microorganisms (Fig. 5). One of the major limitations is the minuscule volume of fluid present in these phagocytotic vacuoles. Neutrophils release large quantities of myeloperoxidase into these vacuoles, while eosinophils release eosinophil peroxidases. Chlorine and bromine are well-established substrates for these enzymes, being oxidized to hypohalous acids. Even though chloride is $100 \mathrm{mM}$ in extracellular fluids, the minuscule volume absorbed into these vacuoles implies that chlorine can be a major limitation in the myeloperoxidase reaction. It has also long been puzzling why the NADPH oxidase of neutrophils produces superoxide rather than hydrogen peroxide directly. The ability of NO to rapidly diffuse across cellular membranes greatly expands the armamentarium of phagocytic inflammatory cells. The large localized production of superoxide will form a major sink to trap NO produced by any cell in the region and the peroxynitrite will react quickly in the immediate region because of the large local concentrations of carbon dioxide produced by activation of the hexose monophosphate shunt activated to supply NADPH needed for superoxide formation and NO synthesis.

Myeloperoxidase reacts rapidly and directly with peroxynitrite to produce nitrogen dioxide and efficiently catalyzes tyrosine nitration $(400,1112)$. 
When peroxynitrite acts as an oxidant, it produces nitrite and hydroxide ion rather than isomerizing to nitrate. Consequently, the major decomposition products of superoxide and peroxynitrite formation in the phagosome are ultimately hydrogen peroxide and nitrite. These are also substrates for myeloperoxidase and can be a significant source of tyrosine nitration $(158,668,1113)$. Using mass spectrometry to quantify nitrotyrosine formation in knockout mice for myeloperoxidase and eosinophil peroxidase, Brennan et al. (136) found that nitrotyrosine was reduced by a maximum of $50 \%$ in some models of severe acute inflammatory but nitration was hardly affected in other models. Nitration catalyzed by peroxidases from nitrite has been frequently interpreted as implying nitrotyrosine does not necessarily result from peroxynitrite. In many cases, peroxidases from inflammatory cells are not found in regions showing tyrosine nitration. Furthermore, a major issue is where does nitrite come from in vivo. NO is mostly removed by reacting with oxyhemoglobin to form nitrate, with only a small fraction being oxidized by heme proteins. Even the highest fluxes of NO proposed to be present in vivo will form minor amounts of nitrogen dioxide by the reaction with oxygen (Fig. 2). By cogenerating superoxide, $\mathrm{NO}$ is rapidly redirected to the initial formation of peroxynitrite. Within a neutrophil or macrophage phagolysosome, this serves as a recycling mechanism to reutilize nitrite and hydrogen peroxide to generate more reactive species. It will be the rare exception to find nitrotyrosine being formed without peroxynitrite being a major intermediate.

\section{The Reverse Reaction of Peroxynitrite to Form Superoxide and Nitric Oxide}

Although the back reaction is $\sim 100,000,000,000$ times slower than the formation of peroxynitrite, the reverse reaction of peroxynitrite forming superoxide and NO can be significant when working with bolus additions. When the production of superoxide and NO are both occurring at very low levels, the back reaction can be significant. However, the rapid reaction of peroxynitrite with carbon dioxide will pull the overall reaction to form carbonate radical and nitrogen dioxide. Thus carbon dioxide increases the amount of radical damage produced from NO plus superoxide cogeneration and ensures that peroxynitrite decomposes to form radicals even at very low fluxes of superoxide and NO.

The reverse reaction to form superoxide and $\mathrm{NO}$ also explains many of the puzzling aspects of peroxynitrite chemistry at alkaline $\mathrm{pH}$ (Fig. 6). The apparent radical generation from peroxynitrite decomposition decreases at alkaline $\mathrm{pH}(75)$. The details have been worked out by Goldstein, Meyreni, Lymar, Hurst, and co-workers $(224,452,812,868)$. In brief, the superoxide produced during the reverse reaction reacts with nitrogen dioxide to form peroxynitrate anion $\left(\mathrm{O}_{2} \mathrm{NOO}^{-}\right)$, which decomposes to give nitrite and oxygen. The NO produced reacts with additional nitrogen dioxide to form dinitrogen trioxide, which is a wellknown nitrosating agent. Peroxynitrite reacts quickly with dinitrogen trioxide to form two nitrogen dioxides plus nitrite. This catalytically increases the formation of nitrogen dioxide and accelerates the decomposition of peroxynitrite. This complex interplay of reactive intermediates illustrates how a slight excess of $\mathrm{NO}$ can interact with peroxynitrite to increase the formation of nitrosothiols and other unexpected products that greatly complicate the biological chemistry of peroxynitrite.

\section{PEROXYNITRITE-INDUCED CYTOTOXICITY}

Under physiological conditions, the production of peroxynitrite will be low and oxidative damage minimized by endogenous antioxidant defenses $(1058,1059)$. Even modest increases in the simultaneous production of superoxide and NO will greatly stimulate the formation of peroxynitrite; a 10-fold increase in superoxide and NO production will increase peroxynitrite formation 100-fold. Consequently, pathological conditions can greatly increase the production of peroxynitrite. Even the generation of a moderate flux of peroxynitrite over long periods of time will result in substantial oxidation and potential destruction of host cellular constituents, leading to the dysfunction of critical cellular processes, disruption of cell signaling pathways, 
and the induction of cell death through both apoptosis and necrosis (1334). Hence, the production of peroxynitrite can be instrumental in the development of many pathological processes in vivo.

\section{A. Mechanisms of Peroxynitrite-Mediated Oxidations}

Although not a free radical in nature, peroxynitrite is much more reactive than its parent molecules $\mathrm{NO}$ and $\mathrm{O}_{2}{ }^{--}(71,75,78)$. The half-life of peroxynitrite is short $(\sim 10-20 \mathrm{~ms})$, but sufficient to cross biological membranes, diffuse one to two cell diameters (312), and allow significant interactions with most critical biomolecules (1041). Kinetic studies have indicated that peroxynitrite oxidizes target molecules through two distinct mechanisms. First, peroxynitrite and its protonated form peroxynitrous acid $(\mathrm{ONOOH})$ can exert direct oxidative modifications through one- or two-electron oxidation processes (78). Only a few chemical groups directly react with peroxynitrite, which favors selective reactions with key moieties in proteins, such as thiols, iron/sulfur centers, and zinc fingers (78). The second mechanism involves peroxynitrite indirectly mediating oxidation by decomposing into highly reactive radicals (16). Peroxynitrite can yield hydroxyl radical and nitrogen dioxide $\left(\mathrm{NO}_{2} \bullet\right)$ during the homolytic decomposition of peroxynitrous acid (1054). However, the formation of hydroxyl radical by this mechanism probably plays only a minor role in vivo (1054) because of the particularly rapid reaction of peroxynitrite with carbon dioxide $\left(\mathrm{CO}_{2}\right)(310,809-811,1060)$. The direct reaction of peroxynitrite with $\mathrm{CO}_{2}\left(4.6 \times 10^{4} \mathrm{M}^{-1} \cdot \mathrm{s}^{-1}\right.$ at $\left.37^{\circ} \mathrm{C}\right)$ gives rise to an unstable product (nitrosoperoxycarbonate, $\mathrm{ONOOCO}_{2}^{-}$), which rapidly homolyzes into the $\mathrm{CO}_{3}^{-} \bullet$ (carbonate radical) and $\mathrm{NO}_{2} \bullet$ (34). Carbonate radical is likely to be more toxic than hydroxyl radical and yields many of the same types of oxidation commonly attributed to hydroxyl radical. Thus carbon dioxide redirects much of the peroxynitrite produced in vivo towards radical mechanisms (16).

\section{B. Biological Targets of Peroxynitrite}

\section{Proteins}

\section{A) REACTIONS OF PEROXYNITRITE WITH TRANSITION METAL CENTERS:}

The direct reaction of peroxynitrite with transition metal centers is among the fastest known for peroxynitrite (16). Peroxynitrite thus modifies proteins containing a heme prosthetic group, such as hemoglobin (106), myoglobin (540), or cytochrome $c$ (1275), oxidizing ferrous heme into the corresponding ferric forms. Similarly, peroxynitrite can inactivate inducible NOS by oxidative modification of its heme group (582), a reaction which might serve as a feedback negative regulation of peroxynitrite generation under inflammatory conditions. Peroxynitrite reacts particularly rapidly with iron-sulfur clusters, inactivating enzymes involved in critical metabolic processes, including mitochondrial aconitase (182) and phoshogluconate dehydratase (669), as well as with $\mathrm{Zn}^{2+}$ sulfur motifs, an effect resulting in the inactivation of eNOS (1469) and alcohol dehydrogenase (245).

B) REACTIONS OF PEROXYNITRITE WITH AMINO ACIDS: I) Cysteine oxidation: Peroxynitrite may alter protein structure and function by reacting with various amino acids in the peptide chain. The most prevalent reaction is that with cysteine, making thiol oxidation a major modification introduced by peroxynitrite $(1056,1057)$. The direct second-order reaction of peroxynitrite with thiols (particularly with the anion form, $\mathrm{RS}^{-}$) results in the formation of an intermediate sulfenic acid (RSOH), which then reacts with another thiol, forming a disulfide (RSSR) (16). Thiols may also be oxidized by the radicals formed from peroxynitrite, generating thiyl radicals $(\mathrm{RS} \bullet$ ). Thiyl radicals may react with oxygen and promote oxidative stress by propagating free radical reactions (334). They will also react with NO to form nitrosothiols. 
The oxidation of critical cysteine residues by peroxynitrite inactivates many enzymes involved in cellular energetic processes, including glyceraldehyde-3-phosphate deshydrogenase (157, 1192), creatine kinase (703), complex I (NADH dehydrogenase), complex II (succinate dehydrogenase), and complex III (cytochrome $c$ reductase) as well as complex V (ATP synthase) from the mitochondrial respiratory chain $(1058,1059,1062)$. These enzymes are also inactivated by nitration of tyrosine residues (see below), indicating that they may be particularly vulnerable targets of peroxynitrite. Cysteine oxidation also inactivates several protein tyrosine phosphatases, which may enhance phosphotyrosine-dependent signaling $(791,1254)$. In addition to protein-bound thiol, peroxynitrite can directly oxidize low-molecular-weight thiols, most notably reduced glutathione (GSH). GSH thereby serves as an efficient endogenous scavenger of peroxynitrite and plays a major role in the cellular defense against this species (31). Accordingly, the susceptibility of cells to peroxynitrite toxicity largely depends on the amount of intracellular GSH. GSH depletion enhances peroxynitrite toxicity and tissue injury during circulatory shock $(258,278)$, and a relationship between GSH depletion and enhanced peroxynitrite toxicity has also been proposed as contributing to the development of some neurodegenerative diseases such as Parkinson's disease and amyotrophic lateral sclerosis $(847,1321)$.

In some instances, cysteine oxidation by peroxynitrite may result in enzyme activation instead of inhibition, as demonstrated for matrix metalloproteinases (MMPs), which have been recently implicated as an important mechanism of peroxynitrite-dependent toxicity in heart disease $(994,1085,1345)$ and stroke (495). Peroxynitrite activates the proenzyme (proMMPs) via thiol oxidation and $S$-glutathiolation of its autoinhibitory domain (971). Similarly, cysteine oxidation by peroxynitrite has been associated with the activation of the src kinase $h c k$ in erythrocytes, inducing the amplification of tyrosine-dependent signaling in these cells, as detailed in section $\mathrm{V}(838,840)$.

II) Tyrosine nitration: Protein tyrosine nitration is a covalent protein modification resulting from the addition of a nitro $\left(-\mathrm{NO}_{2}\right)$ group adjacent to the hydroxyl group on the aromatic ring of tyrosine residues (464). Tyrosine nitration affects protein structure and function, resulting in the generation of antigenic epitopes, changes in the catalytic activity of enzymes, altered cytoskeletal organization, and impaired cell signal transduction (1132), and is thus increasingly considered as a central aspect of peroxynitrite-mediated cytotoxicity.

Tyrosine does not react directly with peroxynitrite. Instead, tyrosine nitration occurs through a radical mechanism in which a hydrogen atom is first abstracted from tyrosine to form a tyrosyl radical that quickly combines with $\mathrm{NO}_{2} \bullet$ to produce 3-nitrotyrosine. This competes with a secondary reaction where combining with another tyrosyl radical to form dityrosine $(72,78$, $605,1053)$. Although the radicals involved in the reaction may come from peroxynitrite homolysis $\left(\mathrm{HO} \bullet\right.$ and $\mathrm{NO}_{2} \bullet$ ), they most likely result from the reaction between peroxynitrite and $\mathrm{CO}_{2}$ (producing $\mathrm{CO}_{3}^{-} \bullet$ and $\mathrm{NO}_{2} \bullet$ radicals) $(16,1053,1300)$. Nitration is also enhanced in the presence of transition metals due to the formation of secondary radicals at the metal center plus $\mathrm{NO}_{2} \bullet$ (16). Metalloproteins such as heme-containing proteins (e.g., prostacyclin synthase) or $\mathrm{Cu}, \mathrm{Zn}$ - and $\mathrm{Mn}-\mathrm{SOD}$ may thus catalyze peroxynitrite-mediated tyrosine nitration (1053). A second mechanism of tyrosine nitration relies on the generation of the $\mathrm{NO}_{2} \bullet$ radical by various heme-peroxidases (mainly myeloperoxidase and eosinophil peroxidase) in the presence of hydrogen peroxide $(438,1314,1390)$. This may serve as a recycling mechanism to reuse products formed from superoxide and NO production. Therefore, nitrotyrosine formation technically must be considered a marker of "nitrative" stress. Additional control experiments are needed before concluding tyrosine nitration resulted from the generation of peroxynitrite (606). 
The advent of proteomic analyses has confirmed that nitration is a highly selective process, limited to specific tyrosine residues on a surprisingly small number of proteins $(35,464,654$, 655). Nitration on specific tyrosine residues is promoted by the exposure of the aromatic ring to the surface of the protein, the location of the tyrosine on a loop structure, its association with a neighboring negative charge, and absence of proximal cysteines (1190). Tyrosine nitration may also be favored in hydrophobic environment, which is a relevant issue, considering that peroxynitrous acid can readily pass through lipid membranes $(64,1300)$.

A) Selected cytotoxic processes related to protein tyrosine nitration: The list of proteins being nitrated in cellular models, as well as in various pathologies in vivo, is rapidly growing. Tyrosine nitration has been identified in at least 50 human diseases and more than 80 conditions modeled in animals, as reviewed recently (476), and these figures are continuously increasing. However, finding of nitrated proteins in a given condition does not necessarily imply a direct pathogenic role, but at least it indicates an increased formation of peroxynitrite along with other nitrogen reactive species during the disease process. Novel proteomic approaches are being used to identify which proteins are nitrated in vivo, and such studies will help to define the potential pathogenic role of tyrosine nitration in diseases.

In most reported studies, nitration of tyrosine has been associated with a significant loss of function of the nitrated protein, as summarized in Table 1. An important example of loss of enzyme activity is that of mitochondrial Mn-SOD, which was the first protein found to be nitrated in vivo. Nitration of a single tyrosine residue (Tyr-34) leads to complete enzyme inactivation (830), with the possible consequence to favor peroxynitrite generation in this organelle, due to the impaired dismutation of $\mathrm{O}_{2}{ }^{--}$. In vivo, nitration of Mn-SOD has been detected in rodent (828) and human (826) rejected kidney allographs, in cerebrospinal fluid of patients with amyothrophic lateral sclerosis as well as Alzheimer's and Parkinson's diseases (27), and in hearts from humans with diabetes (1397) and from mice exposed to cigarette smoke (691), and it has also been associated with vascular aging (1311).

Prostacyclin synthase ( $\mathrm{PGI}_{2}$ synthase) is another important target of peroxynitrite that is inactivated by a specific nitration of Tyr-430 (1130). PGI $_{2}$ synthase is rapidly nitrated in arterial walls during inflammatory processes (39), through a mechanism involving CD40 liganddependent increases in vascular peroxynitrite generation (290). The consecutive loss of $\mathrm{PGI}_{2}$ synthesis may be a significant contributor to endothelial dysfunction in many pathological conditions, e.g., diabetes (1473), atherosclerosis (290), and ischemia-reperfusion (1468) and may play an important role in the phenomenon of nitrate tolerance (549).

In the heart, nitration of several critical proteins has been proposed as a major mechanism of cardiac dysfunction $(995,1300)$. Thus both creatine kinase $(740,877,878,883)$, a critical energetic controller of cardiomyocyte contractility, and the sarcoplasmic reticulum $\mathrm{Ca}^{2+}$ ATPase (SERCA2A) $(789,1397)$ are rapidly inactivated by tyrosine nitration. Peroxynitrite also nitrates and inactivates the voltage-gated $\mathrm{K}^{+}$channels in the coronary endothelium, which may foster cardiac dysfunction by imparing coronary flow reserve (754), and nitrates several important structural protein in cardiomyocytes such as desmin, myosin heavy chain, and $\alpha$ actinin with potential deleterious effects on contractility $(117,878,881)$.

Tyrosine nitration represents a major cytotoxic pathway in the nervous system, possibly contributing to neurodegenerative disorders. $\alpha$-Synuclein, a neuronal presynaptic protein, undergoes oligomerization upon peroxynitrite-mediated nitration, forming Lewy bodies, the hallmark of Parkinson's disease (22), and nitrated $\alpha$-synuclein has been detected both in experimental and human Parkinson's disease $(443,1042)$. Peroxynitrite further contributes to Parkinson's disease through nitration (and cysteine oxidation) of tyrosine-hydroxylase, the rate-limiting enzyme in the synthesis of dopamine $(103,720,721,1005)$. Peroxynitrite nitrates 
the microtubule-associated tau protein, inducing tau aggregation, a critical mechanism of Alzheimer's disease $(1073,1074)$, while peroxynitrite-mediated nitration of neurofilament L may be involved in the alterations of motor neurons in amyotrophic lateral sclerosis (247).

Disabling of several cytoskeletal proteins by nitration represents a further major cytotoxic effect attributed to peroxynitrite. Tubulin nitration by peroxynitrite or by direct incorporation of free nitrotyrosine has been reported in cell lines derived from intestine (54), neurons (1269), and muscle (199), resulting in the loss of normal physiological functions. Peroxynitrite also disorganizes actin polymerization through actin nitration, and via the nitration of profilin $(658,659)$, an important actin-binding protein. These effects have been associated with platelet dysfunction (659), disruption of both intestinal (55) and endothelial barrier function (940), as well as impaired migration and phagocytosis of activated polymorphonuclear cells (221).

Two additional important aspects of tyrosine nitration include the modulation of tyrosine kinase-dependent signaling, which will be exposed in detail in the specific section on peroxynitrite and cell signaling, and the generation of new epitopes on proteins, to which $\mathrm{T}$ and B lymphocytes are not rendered tolerant. A number of nitrotyrosine-carrying proteins have thus been shown to elicit both humoral and cellular immune responses in mice $(102,966)$, and recent findings indicate that nitrated proteins may be involved in the development of autoimmune diseases such as systemic lupus erythematosus, arthritis, and glomerulonephritis $(571,671)$.

III) Tryptophan, methionine, and histidine oxidation: Peroxynitrite directly oxidizes methionine, forming methionine sulfoxide, and to a lesser extent, ethylene and dimethyldisulfide (16). These modifications may participate in immune defenses by inactivating glutamine synthetase (90) and the molecular chaperone GroEL (673) in bacteria. Met oxidation also inhibits $\alpha 1$-anti-proteinase, which then loses its ability to inactivate proteases, most notably elastase (1368). Methionine oxidation is reversed by methionine sulfoxide reductase, an enzyme whose reduced expression in the brain is associated with the development of Alzheimer's disease (1199). Peroxynitrite can also oxidize tryptophan (16), yielding $N$-formylkynurenine, oxindole, hydropyrroloindole, and nitrotryptophan, but the relevance of these effects remains to be established $(16,1403)$. Finally, peroxynitrite modifies histidine through a radical mechanism, forming a histidinyl radical, a mechanism involved in the inactivation of $\mathrm{Cu}, \mathrm{Zn}-\mathrm{SOD}$ by peroxynitrite $(15,1403,1404)$.

2. Lipids-A major aspect of peroxynitrite-dependent cytotoxicity relies on its ability to trigger lipid peroxidation in membranes (1055), liposomes, and lipoproteins by abstracting a hydrogen atom from polyunsatturated fatty acids (PUFA). Resulting products include lipid hydroperoxyradicals, conjugated dienes, and aldehydes (311). Such radicals in turn attack neighboring PUFAs, generating additional radicals which propagate free radical reactions and the degeneration of membrane lipids $(560,1055)$, causing membrane permeability and fluidity changes with significant biological consequences (1075). Peroxynitrite may play a critical role in inflammatory diseases of the nervous system by initiating peroxidation of myelin lipids, leading to demyelination $(1155,1175,1313)$. It also acts as a potent oxidizing agent towards low-density lipoprotein (LDL) $(742,1287)$. Peroxynitrite-modified LDL binds with high affinity to scavenger receptors leading to the accumulation of oxidized cholesteryl esters and foam cell formation, which represent a key early event in atherogenesis $(465,498,558)$. Finally, the interactions of peroxynitrite with membrane lipids may lead to the formation of various nitrated lipids, with potential biological properties as mediators of signal transduction both under physiological and pathological conditions (50), and of several intermediates products, including isoprostanes and 4-hydroxynonenal that can further trigger secondary oxidative insults (311). 
3. Nucleic acids-Peroxynitrite can damage DNA by introducing oxidative modifications in both nucleobases and sugar-phosphate backbone (for review, see Refs. 160,945). Among the four nucleobases, guanine is the most reactive with peroxynitrite due to its low reduction potential (1422). The major product of guanine oxidation is 8-oxoguanine, which further reacts with peroxynitrite, yielding cyanuric acid and oxazolone (945). Ultimately, guanine oxidation by peroxynitrite results in guanine fragmentation, a critical step towards mutagenesis and carcinogenesis $(160,945)$. Peroxynitrite can nitrate guanine, yielding 8-nitro-guanine, which leads to the formation of abasic sites that can be cleaved by endonucleases in vivo to give DNA single-strand breaks $(160,945,1422)$. Peroxynitrite may also attack the sugar phosphate backbone by abstracting a hydrogen atom from the deoxyribose moiety, resulting in the opening of the sugar ring and the generation of DNA strand breaks $(160,945)$. The formation of DNA single-strand breaks represents a critical aspect of peroxynitrite-mediated cytotoxicity, since they represent the obligatory trigger for the activation of the nuclear enzyme poly(ADP-ribose) polymerase (PARP) (1243), a pathway ultimately related to the induction of cell death and tissue inflammation, as developed in detail in the next section.

\section{Peroxynitrite, Mitochondria, and Cell Death}

Mitochondria are involved in many vital processes, e.g., energy production, calcium homeostasis, and the control of various biosynthetic pathways. They also play essential roles in cell death mechanisms. Disruption of mitochondrial functions is implicated in a great number of disease processes, such as diabetes, atherosclerosis, ischemic heart diseases, stroke, aging, and neurodegenerative diseases. The pivotal role of peroxynitrite in such derangements is increasingly recognized, as it can react with key components of mitochondria and thus may affect virtually every critical function of these organelles. Peroxynitrite may reach mitochondria either from extramitochondrial compartments or may be directly produced within the mitochondria. Indeed, mitochondria can produce both $\mathrm{NO}$, by the activity of a $\mathrm{Ca}^{2+}$ sensitive mitochondrial NOS (mtNOS) $(140,528)$, and superoxide, following the partial reduction of oxygen within the mitochondrial matrix due to the natural leak of electron from the respiratory chain. A major physiological function of $\mathrm{NO}$ in the mitochondria is to regulate oxygen consumption by reversibly inhibiting cytochrome- $c$ oxidase (complex IV of the electron transport chain) via competition with oxygen for the binuclear binding site (1001). In conditions of high NO production (e.g., during inflammation, reperfusion injury, or neuronal hyperactivation), the interruption of electron transfer at cytochrome oxidase markedly increases the leakage of electrons from the respiratory chain, resulting in enhanced formation of superoxide within the mitochondrial matrix and generation of significant amounts of peroxynitrite $(143,147,533)$. In turn, peroxynitrite nitrates and inhibits Mn-SOD (830), thereby preventing the breakdown of locally produced superoxide, which further fuels the formation of peroxynitrite. The mitochondrial toxicity of peroxynitrite results both from direct oxidative reactions and from free radical-mediated damage $(1058,1059)$, secondary to peroxynitrite reacting with $\mathrm{CO}_{2}$, giving rise to $\mathrm{CO}_{3}^{-} \bullet$ and $\mathrm{NO}_{2} \bullet$ radicals. The latter reaction is particularly favored within mitochondria, which are the main organelles where $\mathrm{CO}_{2}$ is produced during decarboxylation reactions $(1058,1059)$.

With the use of both cultured cells and isolated sub-mitochondrial fractions, peroxynitrite has been shown to exert significant inhibition to most components of the electron transport chain, including complex I (NADH dehydrogenase) $(146,919,1016)$, complex II (succinate dehydrogenase) $(111,1092)$, complex III (cytochrome $c$ reductase) $(489,1016)$, and complex V (ATP synthetase) $(177,1058,1059)$, through mechanisms involving, to various extents, cysteine oxidation, tyrosine nitration, and damage of iron sulfur centers, as extensively reviewed in References 1058 and 1059. In contrast, cytochrome-c oxidase (complex IV), which is readily inhibited by NO, appears remarkably resistant to peroxynitrite, and may even serve, in its reduced form, as an endogenous catalyst of the two-electron reduction of peroxynitrite 
into nitrite $(1015,1017)$. Another target of peroxynitrite is cytochrome $c$, the nitration of which significantly impairs its redox properties. Notably, cytochrome $c$ nitration increases its peroxidatic activity, leading to the generation of hydrogen peroxide and exacerbation of oxidative damage to mitochondrial proteins $(178,627)$. Peroxynitrite further impairs energy metabolism by inhibiting the tricarboxylic acid cycle enzyme aconitase, located in the mitochondrial matrix, via oxidative disruption of the $4 \mathrm{Fe}-4 \mathrm{~S}$ center of the enzyme $(182,511)$, as well as mitochondrial creatine kinase, which is present in the intermembrane space (1197). Nicotinamide nucleotide transhydrogenase, which allows formation of NADPH from NADH and $\mathrm{NADP}^{+}$, is another important mitochondrial protein oxidized, nitrated, and inactivated by peroxynitrite (403). The ensuing depletion of NADPH reduces the mitochondrial ability to regenerate GSH, contributing to the amplification of oxidative stress within the organelle.

1. Peroxynitrite and apoptosis-Once the level of cellular damage inflicted by peroxynitrite supercedes any possibility of repair, the cell eventually dies via one of the two main pathways of cell demise, necrosis or apoptosis. Necrosis is associated with loss of cellular ATP, leading to membrane disruption, release of noxious cellular debris, and the development of secondary inflammation. In contrast, apoptosis occurs in a well-choreographed sequence of morphological events characterized by nuclear and cytoplasmic condensation with blebbing of the plasma membrane. The dying cell eventually breaks up into membrane-enclosed particles termed apoptotic bodies, which are rapidly ingested and degraded by professional phagocytes or neighboring cells, without inducing any inflammatory response. Apoptosis is orchestrated by the proteolytic activation of cysteine proteases known as caspases, that requires preserved ATP levels to proceed properly, and which may be triggered either by the activation of death receptors (extrinsic pathway) or by the permeabilization of the outer membrane of mitochondria (intrinsic pathway) (see Refs. 121,942 for recent reviews on the topics of apoptosis). The mechanisms of peroxynitrite-mediated apoptotic and necrotic cell death are presented in Figure 7.

While initial studies looking at peroxynitrite-mediated cell death pointed to necrosis as the main death pathway triggered by this species, it soon became evident that apoptosis was also a characteristic consequence of peroxynitrite exposure (115). An early study by Salgo et al. (1105), indicating peroxynitrite's ability to trigger apoptosis in cultured thymocytes, was rapidly followed by numerous reports confirming similar results in virtually every cell type examined. For example, peroxynitrite has been associated with apoptosis in HL-60 cells (773), PC12 cells (367), fibroblasts (1064), SN 4741 dopaminergic neurons (1148), SH-SY5Y neuroblastoma cells (1101), primary neurons $(115,370,664,665)$, astrocytes (1452) and oligodendrocytes (1439), endothelial cells $(319,1339)$, beta islet cells $(309,1210)$, neutrophils $(408,1267)$, chondrocytes (1366), cardiomyocytes $(30,750)$, and renal tubular cells (13).

Several mechanisms have been proposed to explain the activation of the apoptotic program by peroxynitrite, which appear largely dependent on the cell type studied and the experimental conditions (1334). However, a common pathway involving the mitochondria and permeabilization of their outer membrane is emerging as a key feature of peroxynitritemediated apoptosis. Mitochondrial outer membrane permeabilization (MOMP) allows the efflux of various proapoptotic signaling molecules, which promote cell death via both caspasedependent (e.g., cytochrome $c$, APAF-1, Smac/DIABLO) and caspase-independent (especially apoptosis-inducing factor) mechanisms $(121,533)$. MOMP may either be triggered by the formation of pores within the outer membrane by proapoptotic proteins (e.g., Bid, Bax, and Bak), a process inhibited by antiapoptotic Bcl-2 and bcl-XL proteins, or by a phenomenon termed mitochondrial permeability transition (MPT) (29), which is a prominent feature of peroxynitrite-mediated cell death. MPT describes the permeabilization of the inner mitochondrial membrane by a multi-protein complex termed the permeability transition pore, which is composed of the adenine nucleotide translocase (ANT), cyclophilin D (CyP-D) and 
the voltage-dependent anion channel (VDAC). Formation of the permeability transition pore is triggered by calcium overload or by oxidative modifications of critical thiol groups within the ANT, allowing its interaction with CyP-D. The permeability transition pore results in the dissipation of mitochondrial membrane potential $\left(\Delta \psi_{\mathrm{m}}\right)$, cessation of electron transfer and ATP production, and the secondary production of reactive oxygen species within the mitochondria, which further amplify the phenomenon. Finally, the permeability transition pore induces mitochondrial swelling and rupture of the outer membrane with subsequent efflux of the abovementioned proapoptotic molecules. Depending on the degree of MPT, cells may either recover (minimal MPT) or die by apoptosis (moderate or transient MPT, with maintained ATP production) or necrosis (widespread and irreversible MPT, leading to severe ATP depletion) $(982,984,1356)$.

Permeability transition pore opening in response to peroxynitrite has been documented in isolated mitochondria $(118,142,1127,1326)$, where it is likely to occur as a consequence of peroxynitrite-mediated oxidation of cysteine-bound thiols in the ANT (1326). Recent indications that calcium overload enhances PTP opening by peroxynitrite suggest that calciumdependent sensitization of certain mitochondrial proteins to oxidative/nitrative damage is critical for apoptosis to proceed following peroxynitrite exposure (142). At the cellular level, dissipation of mitochondrial potential $(216,758,1148,1332,1339)$, mitochondrial efflux of cytochrome $c(758,1339)$, and caspase activation $(1148,1339)$ occurred to various extents in different cells exposed to endogenously produced or exogenously added peroxynitrite, for example, PC12 cells (758), astrocytes (216), thymocytes (1332), and cardiomyocytes (30) (750). The finding that these events can be prevented by the upregulation of the antiapoptotic protein Bcl-2 $(1148,1326,1332)$ further emphasizes the essential role of mitochondria in peroxynitrite-triggered apoptosis. According to recent results, permeability transition pore opening secondary to peroxynitrite can also elicit AIF efflux and caspase-independent cell death $(1436,1451)$. Gabor Szabo's group (82) has proposed that this mechanism might participate to the phenomenon of vascular restenosis, a serious complication of arterial endarterectomy that is due to neointima proliferation.

In addition to directly targeting the mitochondria, peroxynitrite can also activate cell death mechanisms through the modulation of various cell signal transduction processes. The role of mitogen-activated protein kinases (MAPKs) and Akt (protein kinase B) deserves some comment here, though more details on these cascades are given in section V. MAPKs comprise three distinct members, ERK, JNK, and p38, whose activation regulates many critical cellular functions, notably apoptosis, and which are strongly activated by peroxynitrite in vitro. The peroxynitrite-mediated activation of ERK committed bronchial (922) and neural cells (645, 1099,1420 ) to apoptotic cell death, while activation of JNK, p38, or both triggered a similar outcome in murine alveolar cells (1162), cerebrocortical neurons (120), and PC12 cells (1148), respectively. Recent reports have shown that the release of free $\mathrm{Zn}^{2+}$ by peroxynitrite, possibly due to oxidation of $\mathrm{Zn}^{2+}$-sulfur bridges in mitochondrial and cytosolic proteins $(120,245,1469)$, could play an essential role in initiating these responses $(120,1439,1440)$. Indeed, treatment with zinc chelators impaired ERK $(1439)$ and p38 $(120,1439,1440)$ activation and attenuated peroxynitrite-mediated apoptosis in cells from glial (1439) and neuronal $(120,1440)$ origin. In contrast to the activation of MAPKs, peroxynitrite has been associated with significant inhibition of protein kinase B (Akt), a serine-threonine protein kinase whose activation represents a powerful protective mechanism to limit apoptosis in various stress conditions, including oxidative stress (848). Peroxynitrite blocked the activation of Akt in macrophages (536), adipocytes (949), PC12 cells (1148,1193), and endothelial cells $(353,485-487,1471)$, through a mechanism involving nitration and inactivation of phosphatidylinositol 3-kinase, the upstream signaling intermediate in the Akt pathway (353, 536). In several of these cell systems, overexpression of a constitutively active Akt completely 
masked the proapoptotic effects of peroxynitrite $(353,1148)$, implicating a direct link between Akt inhibition and apoptosis in these experimental conditions.

2. Peroxynitrite and necrosis: the role of PARP-Whereas apoptosis is a typical consequence of low to moderate concentrations of peroxynitrite, exposure of cells to higher concentrations of the oxidant has been associated with necrosis $(115,1334)$. Studies investigating this process have established that peroxynitrite-dependent cell necrosis is not a purely passive phenomenon, but instead is mediated by a complex process involving DNA damage and activation of the DNA repair enzyme PARP-1 (1243). PARP-1 is a member of the PARP enzyme family consisting of PARP-1 and many additional poly-(ADP-ribosylating) enzymes. PARP-1 detects and signals DNA strand breaks induced by a variety of genotoxic insults, including ionizing radiations, alkylating agents, oxidants (essentially hydrogen peroxide, peroxynitrite, and possibly nitroxyl anion), and free radicals (mainly carbonate or hydroxyl radical) $(299,696,1230)$. Upon binding to DNA strand breaks, PARP transfers ADPribose units from the respiratory coenzyme nicotinamide adenine dinucleotide $\left(\mathrm{NAD}^{+}\right)$to various nuclear proteins (e.g., DNA polymerase, histones, and an automodification domain of PARP itself), building up large ADP-ribose polymers that may reach up to 200 units (1150). These posttranslational modifications are transient in nature, and the modified proteins are rapidly restored to their native state by the activity of the enzyme poly(ADP-ribose) glycohydrolase (PARG) (288). From a physiological view point, PARP-1 activity and poly (ADP)-ribosylation reactions are implicated in DNA repair processes, the maintenance of genomic stability, the regulation of gene transcription, and DNA replication $(539,1150$, $1165)$.

An important function of PARP-1 is to allow DNA repair and cell recovery in conditions associated with a low degree of DNA damage. Upon severe DNA injury, overactivation of PARP-1 depletes the cellular stores of $\mathrm{NAD}^{+}$, an essential cofactor of the glycolytic pathway, the tricarboxylic acid cycle, and the mitochondrial electron transport chain $(762,769,1227$, 1243). As a result, the loss of $\mathrm{NAD}^{+}$leads to a marked decrease in the cellular pools of ATP, resulting in cellular dysfunction and cell death via the necrotic pathway $(503,765)$ (Fig. 7). This intriguing mode of cell response to acute genotoxic stress led Berger (86) to propose the "suicide hypothesis" of PARP activation, which can be regarded as a way to eliminate cells after irreversible DNA injury. Evidence has been gathered that both exogenous and endogenously generated peroxynitrite potently induce DNA strand breakage leading to PARP activation in a variety of cell types, including pulmonary (1239) and intestinal epithelial cells (666), vascular endothelial and smooth muscle cells $(430,1233)$, fibroblasts (1240), macrophages (1462), and cardiomyocytes $(444,987,992)$, to cite just a few examples. These effects have been correlated with steady reductions of cellular NAD ${ }^{+}$and ATP pools and the induction of necrosis, which could be prevented either by pharmacological inhibition or genetic deletion of PARP-1, as reviewed in Refs. 1227,1334. A vast amount of experimental studies have then established that the PARP-1 pathway of cell death plays pivotal roles in tissue injury and organ dysfunction in virtually every disease process accompanied by oxidative/nitrosative stress: ischemia-reperfusion, localized and systemic inflammation, diabetes, and circulatory shock to name but a few (for extensive recent reviews on this topics, see Refs. 248,254,373, $624,821,959,995,997,1228,1245,1306)$. An important point to make clear at this stage is the essential distinction between PARP activation, which triggers necrosis by ATP depletion, and PARP cleavage, which is a typical feature of apoptosis. In fact, the caspase-mediated cleavage of PARP into 89- and 24-kDa fragments inactivates the enzyme by destroying its ability to respond to DNA strand breaks, thereby preventing the loss of cellular ATP associated with PARP activation and thereby allowing the maintenance of the cellular energy essential for the execution of apoptosis. As such, PARP cleavage has been proposed to function as a molecular switch between apoptotic and necrotic modes of cell death $(122,750,792)$. 
Besides its well-described function as an inducer of necrotic cell death, two recently discovered roles of PARP have been described. The first one involves the role of PARP in regulating the mitochondria-to-nucleus translocation of apoptosis-inducing factor (AIF). AIF is a $67-\mathrm{kDa}$ mitochondrial death promoting protein, which induces DNA fragmentation by triggering the activation of a yet unidentified nuclease (1216). Recent results have indicated that PARP-1 activity is essential for AIF to translocate to the nucleus in cells exposed to oxidative stress, as shown by the lack of AIF translocation in cells with genetic suppression or pharmacological inhibition of PARP-1. As such, AIF is currently believed to play an important role in PARP-1dependent cell death $(696,1424)$. Although preliminary, these results support the notion that a nuclear mitochondrial cross-talk dependent on poly(ADP)-ribosylation is critical in determining the fate of oxidatively injured cells (696). According to most recent findings, this cross-talk involves a PARP-1-dependent activation of the MAPK JNK1 via a pathway using members of the tumor necrosis factor (TNF) signaling cascade (RIP1 and TRAF2) (1399). Obviously, further studies are required to clarify this intriguing aspect of PARP1 biology.

The second additional role of PARP-1 is its involvement in the upregulation of inflammatory processes. The absence of functional PARP-1 (either genetic or pharmacological) alleviated the expression of a host of proinflammatory mediators, including cytokines, chemokines, adhesion molecules and enzymes (e.g., iNOS, COX-2), and it also reduced tissue infiltration with activated phagocytes in experimental models of inflammation, circulatory shock, and ischemia-reperfusion (see Refs. 361,765,1230 for review). The proinflammatory function of PARP was initially believed to reflect exclusively its role as an inducer of cell necrosis, which promotes inflammation via the spilling of noxious cellular debris into neighboring tissues. However, this concept was reviewed after the demonstration by Oliver et al. (973) of a functional association between PARP-1 and the proinflammatory transcription factor nuclear factor kappa $\mathrm{B}(\mathrm{NF} \kappa \mathrm{B})$. In vitro, these investigators found deficient $\mathrm{NF} \kappa \mathrm{B}$ transcriptional activation induced by TNF- $\alpha$ in cells genetically deficient in PARP-1 (973). They also reported that the administration of lipopolysaccharide (LPS) in vivo resulted in NF $\kappa \mathrm{B}$ activation in macrophages harvested from PARP wild-type, but not PARP-1 knockout, mice (973). This effect was associated with a massive reduction in the expression of iNOS, TNF- $\alpha$, and interferon (IFN)- $\gamma$, which provided a complete protection against endotoxin lethality in PARP- $1^{-1-}$ animals (973). These seminal observations have been extended to show that PARP-1 further participates to the activation of other essential proinflammatory signaling cascades, including JNK $(1458,1459)$ and p38 MAPKs $(501)$, as well as the transcription factors activator-protein-1 (AP-1), stimulating factor-1 (Sp-1), octamer-binding transcription factor-1 (Oct-1), Yin Yang-1 (YY-1), and signal transducer and activator of transcription-1 (STAT-1) (502).

In summary, a large body of experimental evidence accumulated over the past 15 years indicates that peroxynitrite generation from $\mathrm{NO}$ and $\mathrm{O}_{2}{ }^{--}$represents a major threat in the cellular environment. The damaging potential of peroxynitrite is explained by its peculiar chemistry involving direct oxidation as well as radical-mediated nitration reactions. These properties allow peroxynitrite to significantly alter the function of a considerable number of proteins, to degrade membrane structure by peroxidizing lipids, to turn off crucial metabolic functions within mitochondria, and to inflict serious damage to nucleic acids, activating a major pathway of cell injury and inflammation orchestrated by the nuclear enzyme PARP. Once severe enough to overwhelm repair mechanism, these various cytotoxic effects commit cells to death, either through the necrotic or apoptotic pathway. In the living organism, the aggressive behavior of peroxynitrite is emerging as an essential triggering mechanism in the initiation and progression of a large number of acute and chronic diseases. The current state of knowledge regarding these pathological aspects of peroxynitrite in vivo is explored in the next sections of this review. 


\section{PEROXYNITRITE AND CELL SIGNALING}

The concept of cell signaling defines the ability of cells to detect changes in their environment to generate an appropriate physiological response (1362). In the past few years, significant experimental efforts have been put forward to explore the relationships between cellular oxidative processes and the modulation of cell signal transduction, collectively grouped under the concept of "redox signaling" (848). The early observation that NO could regulate many critical cell signaling processes through $S$-nitrosylation of critical cysteine residues in proteins was a milestone discovery in our understanding of redox regulation of signal transduction (1200). Soon thereafter, the identification of peroxynitrite's ability to nitrate tyrosine residues rapidly focused attention on phosphorylation cascades, as this protein modification was found to inhibit cell signaling processes relying on tyrosine phosphorylation. Although this view was initially strongly considered, it proved to be overly simplistic, as peroxynitrite often promoted phosphotyrosine signaling in many instances. Further evidence was gathered that, in many different cell systems in vitro, peroxynitrite behaved as a potent modulator of an array of cell signal transduction pathways, independently from its ability to nitrate tyrosine. After a brief summary of the main cell signal transduction pathways, these emerging aspects of peroxynitrite biology are discussed in detail.

\section{A. An Overview of Cell Signal Transduction}

Most extracellular signals are sensed by two major families of cell membrane receptors, $G$ protein-coupled receptors (GPCRs) and receptor tyrosine kinases (1362). GPCRs interact with $\mathrm{G}$ proteins (guanine nucleotide binding proteins), which act on several downstream effectors to generate second messengers such as inositol trisphosphate, cyclic nucleotides, or $\mathrm{Ca}^{2+}$, which in turn modulate the degree of protein phosphorylation. GPCRs also activate small G proteins (Ras and Rho families) that lie upstream of the MAPK superfamily of proteins (see below) (for review, see Refs. 98,497). Receptor tyrosine kinases (RTKs) are transmembrane glycoproteins consisting of at least 13 families, e.g., receptors for insulin and growth factors. Upon binding by specific ligands, RTKs create docking sites for specific phosphotyrosine binding domains to recruit and activate downstream effectors, including Ras-MAPKs, phosphatidylinositol 3-kinase (PI3K), and protein kinase C. RTKs control most fundamental cellular processes such as cell proliferation, differentiation, and cell survival, and abnormal RTK-dependent signaling has been linked to a number of disease processes, notably cancer and cardiovascular diseases (for review, see Refs. 577,1129).

The cornerstone of signal transduction is represented by reversible protein phosphorylation, controlled both at the level of phosphorylation (kinases) and dephosphorylation (phosphatases). Protein kinases transfer a phosphate to specific amino acids (tyrosine, serine, and threonine) and are defined as tyrosine kinases or serine/threonine kinases. Tyrosine kinases comprise receptor and non-receptor tyrosine kinases (RTKs and NRTKs, respectively). NRTKs are activated by GPCRs and RTKs and represent integral components of the signaling cascades triggered by cell surface receptors (1362). The three main members of NRTKs are the Janus kinases (JAKs), involved in cytokine signaling (1067), focal adhesion kinase (FAK), which transmits adhesion-dependent signals (861), and src kinases, which are essential integrators of signals triggered by growth factor receptors, GPCRs, and cytokine receptors $(796,1274)$.

The major subtypes of serine-threonine kinases are protein kinase A (PKA), which modulates cell metabolism, synaptic transmission, and ion channel activity (reviewed in Ref. 382), protein kinase B (PKB), an inhibitor of apoptosis that also plays roles in glucose metabolism and cell proliferation (for review, see Ref. 512), protein kinase C (PKC), involved in the regulation of cell growth and differentiation, cell death, and stress responsiveness (174), and the MAPKs. MAPKs comprise extracellular signal-regulated protein kinase (ERK), which mainly 
transduces signals involved in cell proliferation, p38 MAPK, and c-Jun $\mathrm{NH}_{2}$-terminal kinase (JNK) (652), which are both linked to stress and therefore collectively termed stress-activated protein kinases (SAPKs). The MAPKs have multiple downstream targets, activation of which regulate virtually every critical cellular functions (1048). An additional pathway involves $\mathrm{NF} \kappa \mathrm{B}$, a crucial transcription factor involved in inflammatory and antiapoptotic signaling, which is activated in response to serine phosphorylation of an upstream kinase complex termed IKK (116). The rapid reversibility of phosphorylation is a key point of signaling, catalyzed by three types of protein phosphatases, including tyrosine, serine/threonine, and dual-specificity phosphatases, which have been the matter of several recent reviews $(1126,1206,1283)$.

Dephosphorylation is as tightly regulated as phosphorylation by modulatory proteins that associate with the phosphatases.

\section{B. Modulation of Cell Signaling by Peroxynitrite}

1. Inhibition of phosphotyrosine-dependent signaling-The ability of peroxynitrite to nitrate tyrosine residues can impair signaling processes depending on tyrosine phosphorylation. Early in vitro studies using peptide substrates showed that phosphorylation of critical tyrosine residues within these peptides was markedly inhibited by peroxynitritemediated tyrosine nitration $(463,702)$, and further results indicated that tyrosine nitration blocked downstream signaling in intact cell systems in vitro. In the human neuroblastoma SHSY5Y cells, the peroxynitrite generator SIN-1 triggered the nitration of the focal adhesion protein $130^{\text {cas }}$, resulting in the blockade of its phosphorylation and interfered with the assembly of focal adhesion complexes (1100). Also, peroxynitrite-dependent nitration of a key tyrosine residue (Tyr686) within the cytoplasmic domain of the adhesion molecule plateletendothelial cell adhesion molecule-1 (PECAM-1), interfered with its phosphorylation by src family protein kinases, and prevented its binding to the protein-tyrosine phosphatase SHP-2 (941).

The impairment of tyrosine phosphorylation by peroxynitrite may affect various fundamental cellular functions. For example, in T lymphocytes, peroxynitrite triggered widespread protein nitration and blocked tyrosine phosphorylation in response to cell activation through the T-cell receptor (TCR)/CD3 complex. This resulted in a depressed proliferative response of activated $\mathrm{T}$ cells, suggesting that peroxynitrite might negatively affect normal immune responses depending on T cells in vivo (137). Similarly, nitrotyrosine formation in human platelets inhibited tyrosine phosphorylation in response to thrombin, thereby preventing their activation $(795,896)$. Under certain conditions, competition between nitration and phosphorylation on a single tyrosine residue may completely disrupt a complex chain of signal transduction, as recently shown in primary rat hepatocytes. These cells undergo apoptotic cell death upon stimulation with CD95 (Fas) ligand, through a pathway requiring epidermal growth factor receptor (EGFR) activation followed by EGFR-mediated CD95 tyrosine phosphorylation. Activated CD95 then promotes the formation of a death-inducing signal complex (DISC), committing the cell to apoptosis. In the presence of a short pretreatment with peroxynitrite, CD95 becomes tyrosine nitrated, completely blocking its phosphorylation by activated EGFR, preventing the formation of DISC and the induction of the apoptotic program (1072).

2. Activation of phosphotyrosine-dependent signaling-The initial hypothesis that tyrosine nitration would essentially inhibit phosphotyrosine-dependent cell signaling has been largely reviewed at the light of multiple observations indicating that peroxynitrite promoted, rather than inhibited, tyrosine phosphorylation in a variety of cell types, including red blood cells $(823,836-840)$, bovine brain synaptosomes (318), SH-SY5Y cells (760), pancreatic adenocarcinoma cells (829), and endothelial cells (1470). Furthermore, the realization that peroxynitrite, in a myriad of distinct cell types, strongly activates all MAPK family members (see specific section below), which depend on the upstream activation of various protein 
tyrosine-kinases, further endorsed the ability of peroxynitrite to regulate positively tyrosine phosphorylation.

Two salient features of phosphotyrosine signaling upregulation by peroxynitrite were generally demonstrated: its transient and reversible nature (a typical aspect of cell signaling processes) and its dependence on relatively low bolus additions of 10-200 $\mu \mathrm{M}$, consistent with a signaling role of peroxynitrite in vivo. Equivalent exposures to endogenous peroxynitrite, as measured by the area under the curve, can be achieved in tissues after a few minutes of sustained peroxynitrite formation. In general, higher concentrations enhanced nitrotyrosine formation (which was generally not reversible) and downregulated phosphotyrosine signaling, suggestive of a direct competition between nitration and phosphorylation of tyrosine at high peroxynitrite concentrations. This has been notably well demonstrated with the band 3 protein of human erythrocytes, a membrane anion-exchange protein whose activation results in a cascade of signaling events stimulating glycolytic activity. Peroxynitrite at 10-100 $\mu \mathrm{M}$ activated band 3 Tyr phosphorylation, resulting in the activation of glycolysis, whereas higher concentrations $(200-1,000 \mu \mathrm{M})$ induced band 3 nitration, blocked its phosphorylation, and irreversibly inhibited glycolysis (839). What, then, explains the phosphorylation of tyrosine at (relatively) low peroxynitrite concentrations? As mentioned earlier, the upregulation of phosphotyrosine signaling reflects either the inhibition of phosphotyrosine phosphatases (PTPs) or the activation of phosphotyrosine kinases (PTKs). Recent evidence indicates that peroxynitrite can act on these two levels of regulation.

First, irreversible inhibition of PTPs by very low concentrations of peroxynitrite has been demonstrated both in cells $(791,839)$ and purified enzymes $(175,1254)$. All PTPs contain a conserved cysteine residue, which forms an intermediate phosphocysteine with the phosphatase substrate of the PTP, and oxidation of this critical cysteine has been shown to inactivate the PTPs (1254). Peroxynitrite anion is structurally similar to phosphate anion, so that the extreme vulnerability of PTPs to peroxynitrite-mediated inactivation is consistent with attraction of peroxynitrite to the active site of the enzyme and subsequent oxidation of this essential cysteine (1254).

The second mechanism of phosphotyrosine upregulation by peroxynitrite relies on the direct activation of protein tyrosine kinases. Receptor tyrosine kinases, notably the growth factor receptors EGFR and PDGFR, undergo Tyr phosphorylation upon exposure to oxidants, through mechanisms that remain only partially defined. Tyr phosphorylation and activation of EGFR by peroxynitrite has been reported in human skin fibroblasts (687) and rat lung myofibroblasts (1434), while PDGFR activation occurred in fibroblasts, where it was followed by the downstream activation of the antiapoptotic PI3K/Akt signaling pathway (687). Similarly, peroxynitrite triggered phosphorylation of TrkB, the receptor for brain-derived neurotrophic factor and activated downstream phospholipase C signaling in 3T3 murine fibroblasts (1427). Such activation, however, appears highly cell specific, in view of the contrasted results obtained in other cell systems. Indeed, in A431 epidermoid carcinoma cells, EGFR was shown to dimerize covalently upon treatment with peroxynitrite, with no increased activity in terms of autophosphorylation; rather, the activation by EGF of a downstream molecule, phospholipase C- $\gamma 1$, was attenuated (1316). Also, peroxynitrite-dependent nitration of EGFR prevented its phosphorylation in intestinal Caco-2 cells and blocked cell proliferation in response to EGF stimulation (1301).

Several independent groups have provided strong experimental support implicating the NRTK family member $\mathrm{Src}$ as a preferential target of peroxynitrite. Src family members participate in a variety of signaling processes, including mitogenesis, T- and B-cell activation, cell differentiation and proliferation, as well as cytoskeleton restructuring, through the activation of an array of downstream effectors such as PI3K, phospholipase C, and FAK (1087). 
Activation of src kinases by peroxynitrite has been demonstrated in red blood cells (836838,840 ), brain synaptosomes (837), pancreatic adenocarcinoma cells (829), SH-SY5Y cells (760), bovine endothelial cells (1470), PC12 cells (638), and rat astrocytes (519).

The particular mechanism of regulation of src kinases helps explain why these kinases are so susceptible to the action of peroxynitrite. Src members possess a common structure, with a tyrosine kinase domain and two conserved protein-protein interaction domains, $\mathrm{SH} 2$ and $\mathrm{SH} 3$, which create docking sites for phosphotyrosine and proline-containing sequences, respectively (1087). The catalytic activity of src kinases is tightly regulated in two opposite ways by phosphorylation of two distinct tyrosine residues. Phosphorylation of Tyr-527 within the $\mathrm{COOH}$-terminal domain induces its binding to $\mathrm{SH} 2$, which maintains the kinase in a closed conformation. Dephosphorylation of Tyr-527 by various phosphatases opens the structure and activates the kinase $(796,1087,1274)$. Alternatively, the activation of src is promoted by autophosphorylation of Tyr-416 within the catalytic domain and may further be triggered by oxidation of specific cysteines within the $\mathrm{COOH}$-terminal domain $(10,1045)$.

Two distinct mechanisms of activation of src kinases by peroxynitrite have been identified. In human red blood cells, the src kinase $h c k$ was activated by peroxynitrite via cysteine oxidation, whereas another src kinase, lyn, was activated through a mechanism involving the inhibition of Tyr527 binding to the $\mathrm{SH} 2$ domain $(838,840)$. In fact, several tyrosine-containing peptides were also able to activate lyn when the tyrosine was substituted with 3-nitrotyrosine, by displacing phosphotyrosine 527 from its binding site within the SH2 domain (838). This is not completely surprising, since nitration has the effect of decreasing the $\mathrm{p} K_{\mathrm{a}}$ of phenoxyl groups in free tyrosine from 10 to $\sim 7.2$. This effectively produces a negative charge on the tyrosine and thus mimics the change imposed by phosphorylation (887). Mallozzi et al. (838) proposed the hypothesis that, in erythrocytes, peroxynitrite would preferentially induce nitration of the band 3 protein and that nitrotyrosine-containing band 3 would then divert $\mathrm{SH} 2$ binding to Tyr-527 of lyn, fostering its activation. These findings are of great biological significance, as they suggest that tyrosine nitration may represent a gain-of-function in the regulation of $s r c$ kinases, with potential consequences in several pathological conditions. Indeed, upregulation of $s r c$ kinases may induce rapid and uncontrolled cell growth and transformation, which might link peroxynitrite to carcinogenesis.

3. Peroxynitrite and MAPK signaling-MAPKs (ERK, JNK, and $\mathrm{p} 38$ ) are all activated by a dual phosphorylation at a specific tripeptide motif, mediated by a conserved protein kinase cascade, involving MAP kinase kinase kinases (MKKK or MEKK) and MAP kinase kinases (MKK or MEK) (328). The upstream signaling pathways leading to MKKK activation largely depend on the activation of growth factor receptors and small $\mathrm{G}$ proteins, such as Ras, Rac, and Cdc42 $(328,848)$. Downstream targets of MAPKs include an array of proteins as well as transcription factors, whose activation regulates virtually every critical cellular function, especially apoptosis, cell proliferation, and inflammatory genes expression. The particular mode of MAPK activation, as well as some evidence indicating their activation by peroxynitrite, is depicted in Figure 8.

A) ERK PATHWAY: ERK is involved in the signaling pathways triggered by growth factors and their receptors, via the successive activation of the small G protein ras, Raf- 1 kinase and MEK 1 (1434). ERK can also be activated by various extracellular stresses, including oxidants and free radicals (848). In vitro, peroxynitrite potently activated ERK in fibroblasts (57, 1434), neutrophils (735,1477), endothelial and vascular smooth muscle cells (1307), neural cells $(191,638,645,1099)$, and cardiomyocytes (1024), through strikingly distinct and cellspecific mechanisms. In PC 12 cells, ERK activation occurred via activation of EGFR, in a src tyrosine kinase- and calcium/calmodulin-dependent manner (638), whereas in human neutrophils, ERK activation was associated with the concomitant activation of ras, Raf-1, and 
MEK (1477). We recently provided evidence that peroxynitrite activates ERK in H9C2 cardiomyocytes via the upstream activation of Raf- 1 and MEK, independently from ras and EGFR activation (1024). We proposed that Raf-MEK-ERK activation by peroxynitrite was due to oxidative, but not nitrative, modifications since it was unaffected by epicatechin (1024), a potent inhibitor of nitration reactions (1134). Such findings contrasted with those of Zhang et al. (1434), who found that ERK activation depended entirely on tyrosine nitration of MEK in rat lung myofibroblast. An additional mechanism of ERK activation by peroxynitrite, relying on a direct interaction with a calcium-dependent PKC isoform, has also been reported in rat-1 fibroblasts (57).

Information regarding the consequences of ERK activation in response to peroxynitrite remains limited. In human neutrophils, ERK activation by peroxynitrite (up to $200 \mu \mathrm{M}$ ) has been associated with the upregulation of surface expression of the $\beta_{2}$-integrins CD11b/CD18 and increased neutrophil adhesion to endothelial cells (1477), as well as an enhanced oxidative burst upon stimulation (735). These data then support a potential role of peroxynitrite in mediating excessive neutrophil trafficking and superoxide generation under inflammatory conditions. Another important aspect of ERK activation by peroxynitrite is related to apoptosis. Of note, the role of ERK in apoptosis is not uniform, acting either as an antiapoptotic, or proapoptotic signaling pathway, depending on the kinetics of its activation and on the kind of activating stimulus (848). With respect to peroxynitrite stimulation, it is noticeable that all studies performed so far have indicated a proapoptotic role of ERK. Indeed, peroxynitriteinduced apoptosis in primary rat astrocytes (1420), human SH-SY5Y neuroblastoma cells (1099), human bronchial epithelial BEAS-2B cells (922), and primary murine neurons (645) was associated with the activation of ERK and was significantly attenuated by MEK-ERK pathway inhibitors. The mechanisms linking ERK activation with apoptosis after peroxynitrite stimulation are poorly understood, but may involve a ras/ERK-dependent activation of the proapoptotic protein $\mathrm{p} 53$ in response to peroxynitrite, as demonstrated in primary neural cells (645).

B) JNK PATHWAY: JNK exists as three distinct isoforms, activated in response to many different environmental stresses via a signaling cascade involving the small $\mathrm{G}$ proteins ras and rac, several MAPKKKs, MAPKK, MKK1, and MKK4, linked together by various scaffold proteins in specific signaling modules $(198,291)$. Activated JNK phosphorylates the protoconcogene product c-jun, allowing its homodimerization or heterodimerization with cfos to form the active transcription factor AP-1. JNK is involved in the regulation of inflammation and cell death, with both pro- and antiapoptotic reported functions $(198,291)$.

JNK activation in response to peroxynitrite has been reported in many different cell types $(23,450,922,1024,1128,1162)$, but the mechanisms and outcome of JNK activation have only been addressed in two distinct studies. Go et al. (450) showed that endogenously produced peroxynitrite was responsible for the activation of JNK triggered by laminar shear stress in endothelial cells (450). Importantly, laminar shear stress is a key mechanism protecting blood vessels from the development of atherosclerosis, and JNK activation might be crucial for that function. The study by Go et al. (450) thus has important physiological implications, as it suggests that peroxynitrite could represent a fundamental signaling device connecting mechanical stress to such protective responses. A second study revealed that JNK activation by peroxynitrite was causally linked to apoptotic cell death in murine alveolar C10 cells, as cells expressing a dominant negative mutant of JNK1 were protected from peroxynitritemediated apoptosis (1162). The mechanism of JNK activation involved oxidation and aggregation of the death receptor Fas, followed by the recruitment of Fas-associated death domain (FADD) and subsequent activation of JNK (1162), a signaling cascade which might partly explain the apoptosis of lung epithelial cells occurring in chronic lung inflammatory diseases, where high levels of peroxynitrite have been reported (628). 
C) P38 MAPK: The $\mathrm{p} 38$ family consists of at least five different isoforms: $\alpha, \beta_{1}, \beta_{2}, \gamma$, and $\delta$, whose activation by environmental stress is controlled by several MAPKKKs as well as MKK3 and MKK6. The activation of $\mathrm{p} 38$ has been linked with apoptotic cell death and mitotic arrest in a great variety of cells exposed to different oxidants and free radicals (848). Peroxynitrite is extremely efficient in activating p38, as shown by the very early (within minutes) phosphorylation of $\mathrm{p} 38$ upon peroxynitrite stimulation, even at low concentrations $(<10 \mu \mathrm{M})$ in cardiomyocytes (1024), endothelial $(350,450)$ and vascular smooth muscle cells $(1307)$, hepatocytes $(414,1128)$, bronchial epithelial cells (922), and neural cells $(120,638,965,1120$, 1440). In most of these cell systems, peroxynitrite-mediated $\mathrm{p} 38$ activation could be directly linked to apoptosis, the latter being efficiently prevented by pharmacological inhibitors of p38 $(120,922,965,1120)$. As with other signaling cascades triggered by peroxynitrite, the mechanisms activating $\mathrm{p} 38$ appear as diverse as the cell type studied. Peroxynitrite activated p38 via MKK3 and MKK6 in response to ERK-dependent activation of cytosolic phosholipase $\mathrm{A}_{2}$ in human bronchial epithelial cells (1307), via the upstream activation of calcium/ calmodulin kinase II and src in PC12 cells (638), and via the release of $\mathrm{Zn}^{2+}$ in primary neurons $(120,1440)$. The latter mechanism might be set in motion in various neurological disorders (notably stroke and neurodegenerative disorders), where excessive neuronal NO synthesis and peroxynitrite generation have been associated with enhanced apoptotic cell death in the brain.

4. PI3K/protein kinase B (Akt) pathway-Protein kinase B (Akt) is a serine-threonine protein kinase that plays key roles in integrating cellular responses to growth factors and other extracellular signals. Akt activation also represents an important protective mechanism to limit apoptotic cell death in conditions of oxidative stress (848). The activation of Akt critically depends on PI3K signaling, via the generation of 3'-phosphorylated phosphoinositides (848).

Data regarding the influence of peroxynitrite on the PI3K-Akt pathway are highly controversial. In human skin fibroblasts, peroxynitrite induced Akt activation in a PDGFRand PI3K-dependent manner (687). Similarly, peroxynitrite activated PI3K and Akt phosphorylation in a rat hepatoma cell line, resulting in the activation of the transcription factor Nrf2/ARE and the induction of the detoxifying enzyme glutathione- $S$-transferase (652).

Schroeder et al. (1133) proposed that thiol oxidation, but not tyrosine nitration, underlies the activation of Akt by peroxynitrite, as the latter was not modified by the nitration inhibitor epicatechin in mouse endothelial cells. In striking contrast, several studies concluded that peroxynitrite inhibited PI3K signaling and the activation of Akt in several cells, including Raw 264.7 macrophages (536), 3T3-L1 adipocytes (949), PC12 cells (367,1193), retinal cells (353), and bovine endothelial cells (1471) via a postulated mechanism involving nitration of a critical tyrosine residue within the p 85 regulatory subunit of PI3K $(353,536)$. It is unclear at present why peroxynitrite exerts such a contrasted influence on a single signaling pathway, but the concentration of peroxynitrite, the type of cell under investigation, and most importantly the particular chemical microenvironment are important issues to consider. In particular, the local content of $\mathrm{CO}_{2}$ might represent a crucial factor influencing the outcome of PI3K signaling in response to peroxynitrite, given that reaction between $\mathrm{CO}_{2}$ and peroxynitrite will redirect its chemistry towards radical-mediated nitration reactions (see sect. IV) (1061).

5. PKC pathway-PKC represents a family of phospholipid-dependent serine/threonine kinases involved in signaling pathways regulating cell growth and differentiation, cell death, immune response, transcriptional regulation, and stress responsiveness (notably oxidative stress) (458,848). PKC-mediated cellular effects are both tissue and isoform specific. PKC exists as 11 different isoforms, subdivided in three distinct subgroups (classical PKC $\alpha, \beta \mathrm{I}, \beta \mathrm{II}$, $\gamma$; novel $\operatorname{PKC} \delta, \varepsilon, \eta, \theta$; and atypical $\operatorname{PKC} \lambda, l, \zeta$ ), separated upon their particular mechanism of activation (45). 
In the heart, an NO-dependent activation of PKC- $\varepsilon$ has been implicated in the development of cardioprotection afforded by ischemic preconditioning. A recent study, using both in vitro and in vivo approaches, provided new insights into these mechanisms, by showing that peroxynitrite, rather than NO itself, may be the species responsible for the activation of PKC$\varepsilon$ during preconditioning (52). Peroxynitrite-dependent activation of $\mathrm{PKC}$ has been further reported in endothelial cells, where it has been linked with the activation of cytosolic phospholipase $\mathrm{A}_{2}$ (cPLA2) and an enhanced release of vasoactive mediators (195), a mechanism possibly involved in the phenomenon of nitrate tolerance (5). Alternatively, peroxynitrite has been associated with a significant reduction of the activity of $\operatorname{PKC} \alpha, \beta, \varepsilon$, and $\zeta$ in neuronal cells, and the degree of this inhibition correlated completely with the degree of tyrosine nitration within the enzyme (689). Importantly, PKC is essential for a number of aspects of neuronal functions including synaptic plasticity, learning, and memory. Decreased PKC activity may contribute to several neurodegenerative disorders $(66,689)$, which are also associated with increased peroxynitrite generation (1284). PKC inhibition might thus represent one of the mechanisms linking peroxynitrite in the brain with neurodegeneration.

6. NFKB-NF $\kappa \mathrm{B}$ is a crucial transcription factor activating inflammatory and antiapoptotic genes in response to immunostimulation. $\mathrm{NF} \kappa \mathrm{B}$ is a family of dimeric proteins ( $\mathrm{p} 50$ and its precursor p105, p52 and its precursor p100, p65 or RelA, RelB, and c-Rel) normally held in the cytoplasm in an inactive form, bound to inhibitory proteins, the $\mathrm{I} \kappa \mathrm{Bs}$. The critical step in $\mathrm{NF} \kappa \mathrm{B}$ activation relies on its dissociation from the $\mathrm{I} \kappa \mathrm{B}$ protein, secondary to phosphorylation and proteasomal degradation of $\mathrm{I} \kappa \mathrm{B}$. I $\kappa \mathrm{Bs}$ are phosphorylated by a protein kinase complex, I $\kappa$ B kinase (IKK), composed of two catalytic subunits, IKK $\alpha$ and IKK $\beta$, and a regulatory subunit, IKK $\gamma$ (for review, see Refs. 116,442). The classical ("canonical”) pathway of NFkB activation mainly depends on IKK $\beta$ and triggers transcription of inflammatory and antiapoptotic genes $(205,340,477)$. The alternative pathway ("noncanonical"), which is dependent on IKK $\alpha$, has been shown to be important for B-cell maturation and lymphoid organ development $(306,330,1145)$.

In addition to usual activators of $\mathrm{NF} \kappa \mathrm{B}$ (mainly cytokines and microbial products), there is ample evidence indicating that reactive oxygen species can also trigger this signaling cascade (449). Only a few studies have addressed the potential role of peroxynitrite. A series of elegant studies from Janos Filep's group in Montreal have shown that peroxynitrite, both exogenously added or endogenously produced in response to LPS, cytokines, or Toll-receptor 9 stimulation, potently activated NF $\kappa$ B and stimulated thereby interleukin (IL)- 8 secretion by human polymorphonuclear cells $(391,640,674,1476)$. These studies thus identified an important signaling mechanism by which peroxynitrite amplifies neutrophil-dependent responses under inflammatory conditions. Matata et al. (851) also reported that mononuclear cells exposed to micromolar concentrations of peroxynitrite disclosed $\mathrm{NF} \kappa \mathrm{B}$ activation and a stimulated production of TNF- $\alpha$ and IL- 6 . These authors proposed that nitration of tyrosine- 42 in I $\kappa \mathrm{B}$ might increase its degradation, triggering $\mathrm{NF} \kappa \mathrm{B}$ activity (851).

The stimulating effect of peroxynitrite on NF- $\kappa$ B is however not so straightforward. Two recently published studies rather proposed that this species functions as a potent repressor of $\mathrm{NF} \kappa \mathrm{B}$ activation. Park et al. (1006) found that peroxynitrite eliminated both constitutive and stimulated NF $\kappa$ B activities in P19, SH-SY5Y, and HEK293 cells. Liquid chromatographycoupled nanoelectrospray mass spectrometry revealed specific nitration on Tyr-66 and Tyr-152 residues of p65, leading to 65 destabilization, nuclear export, and inactivation of $\mathrm{NF} \kappa \mathrm{B}$ activity (1006). We further confirmed such observations by demonstrating that a brief exposure to peroxynitrite suppressed $\mathrm{NF} \kappa \mathrm{B}$ activation triggered by LPS and inflammatory cytokines in cardiac H9C2 cells, as well as endothelial EAHY-926 and HMEC-1 cells. The mechanism underlying such inhibition was a complete inhibition of IKK $\beta$ activity in these cells. Intriguingly, whether IKK $\beta$ was suppressed, there was a simultaneous activation of IKK $\alpha$ 
phosphorylation, suggesting that peroxynitrite differentially regulates the classical and the alternative pathways of $\mathrm{NF} \kappa \mathrm{B}$ activation (749). The ramifications of these effects with respect to inflammation are still unclear at present, but the results of the two later studies open the unexpected possibility that peroxynitrite might downregulate the expression of proinflammatory mediators and thereby provide a counterregulatory mechanism to prevent overt inflammation in various pathological conditions, an issue that should be more precisely characterized in years to come.

In summary, peroxynitrite exhibits typical properties of a signaling molecule in vitro, either activating or inhibiting several major signal transduction pathways, which are summarized in Table 2. Initial studies only considered that peroxynitrite would behave as an inhibitor of phosphotyrosine-dependent signaling, owing to its ability to nitrate tyrosine residues within proteins. However, it rapidly turned out that, in many instances, peroxynitrite rather acted to upregulate an array of signaling cascades, by mechanisms involving the inhibition of phosphatases and the direct activation of many different protein kinases. In spite of this experimental evidence, two major difficulties presently limit the interpretation of these findings: their considerable cell specificity and the lack of confirmation in vivo. Further studies must parse the multiple effects triggered by peroxynitrite in culture on the $\mathrm{NF} \kappa \mathrm{B}$ in the intact organism to understand the potential pathological relevance of peroxynitrite on this crucial proinflammatory pathway.

\section{NITRIC OXIDE AND PEROXYNITRITE IN DISEASE}

\section{A. Nitric Oxide and Peroxynitrite in Cardiac Diseases}

Calcium-dependent NOSs are responsible for the generation of NO in cardiomyocytes, endocardial endothelium, coronary endothelium, and cardiac nerves. NO serves many important physiological roles in the regulation of cardiac function including coronary vasodilation, inhibiting platelet and neutrophil adhesion and activation, modulation of cardiac contractile function, and inhibiting cardiac oxygen consumption $(849,1014)$ (Fig. 9). NO is necessary for normal cardiac physiology and plays a protective role in the ischemic heart by numerous mechanisms including stimulation of soluble guanylyl cyclase to decrease of intracellular $\mathrm{Ca}^{2+}$ through activation of cGMP-dependent protein kinase, as well as termination of chain-propagating lipid radical reactions caused by oxidative stress $(636,1149)$ (Fig. 9). NO, through its interactions with components of the mitochondrial respiratory chain, may function as a physiological regulator of cell respiration and a modulator of the generation of reactive oxygen species by mitochondria, thereby affecting mechanisms of cell survival or death (891). In higher levels, or in the presence of reactive oxygen species, NO can also exert cytotoxic effects, potentially through the formation of peroxynitrite (1149). The hypothesis that peroxynitrite generation contributes to myocardial and vascular dysfunction during ischemia and reperfusion (I/R), myocarditis, chronic heart failure, and various other cardiovascular pathologies has been the focus of intensive investigations during the last decade.

\section{Cardiovascular effects of peroxynitrite in vitro and in vivo, potential targets of peroxynitrite-induced toxicity in the cardiovascular system-Experimental} studies utilizing isolated cardiomyocytes, endothelial and smooth muscle cells, papillary muscle, and perfused hearts have generally taken two approaches: exogenous application of peroxynitrite or addition of precursors that enter the cells and generate peroxynitrite endogenously $(320,790,816,1135)$.

Peroxynitrite has been shown to trigger apoptosis in cardiomyocytes $(30,480,750)$ as well as endothelial $(319,879)$ and vascular smooth muscle $(755,756)$ cells, induce decrease in spontaneous contractions of cardiomyocytes (614), and cause irreversible inhibition of the mitochondrial respiratory chain $(148,1383)$. Peroxynitrite also activates ERK, a MAPK which 
has been linked with hypertrophic and antiapoptotic responses in the heart, and inhibits NF $\kappa \mathrm{B}$ activation triggered by inflammatory stimuli in cardiac and endothelial cell lines $(749,1024)$. Furthermore, it induces the upregulation of adhesion molecules in endothelial cells, the disruption of endothelial glycocalyx, and may enhance the adhesion of neutrophils to the endothelium, through complex interactions with various cell signaling pathways (see sects. IV and V) $(424,723,1178,1447,1477)$, and depending on the environment can stimulate or inhibit platelet aggregation $(908,953)$. Peroxynitrite also activates MMPs and nuclear enzyme PARP, which contribute to impaired cardiovascular function in most cardiovascular pathologies and inflammatory disorders discussed below (reviewed in Refs. 624,995,1333).

In isolated rat left ventricular papillary muscle preparations, exogenously applied peroxynitrite, or peroxynitrite generated with NO donor $S$-nitroso- $N$-acetyl-D,L-penicillamine (SNAP) plus superoxide-releasing agent pyrogallol, caused an irreversible inhibition of cardiac contractile and respiratory function $(320,1396)$. Using rat cardiac myofibrils and trabeculae, Mihm and Bauer (877) have demonstrated that the myofibrillar isoform of creatine kinase (a critical energetic controller of cardiomyocyte contractility) is a vulnerable target of peroxynitriteinduced nitration and inactivation in vitro (877). The extent of nitration was linearly related to peroxynitrite concentration and highly correlated to the extent of myofibrillar creatine kinase inhibition (877). Importantly, the creatine kinase-dependent force generation was impaired at peroxynitrite concentrations as low as $50 \mathrm{nM}$, suggesting potent inactivation of the enzyme. Furthermore, the extent of peroxynitrite-induced tyrosine nitration of myofibrillar creatine kinase negatively correlated to myofibrillar creatine kinase-dependent force generation, suggesting that the cardiac contractile apparatus is a highly sensitive target to peroxynitriteinduced injury, and that myofibrillar creatine kinase may be a uniquely vulnerable target (882). Peroxynitrite may also inactivate myocardial aconitase, a critical citric acid cycle enzyme present both in mitochondria and cytosol that converts citrate to isocitrate (211). Furthermore, myocardial $\alpha$-actinin (a cytoskeletal, tyrosine-rich protein crucial for the maintenance of the $\mathrm{Z}$ line and for the integrity of the sarcomeres) and sarcoplasmic reticulum $\mathrm{Ca}^{2+}$-ATPase are also potential targets for peroxynitrite-induced protein nitration in human heart $(117,789)$.

Physiological relevant concentrations of peroxynitrite achieved either by the combination of the NO donor SNAP and pyrogallol (790) or by using the peroxynitrite generator 3morpholinosidnonimine (SIN-1) (816) also induced ventricular dysfunction and myocardial injury of crystalloid perfused (Langendorff) rat hearts which were attenuated with the addition of the antioxidant agent glutathione, SOD, or MMP inhibitor $(816,1345)$. However, infusion of peroxynitrite into the working Langendorff rat heart revealed two contrasting effects dependent on concentration (1136). After exposures to a nominal concentration of $3 \mu \mathrm{M}$, which, due to the short half-life of peroxynitrite, translates into a more actual exposure in the nanomolar concentration range, vascular responses were severely impaired. Specifically, the vasodilatation elicited by acetylcholine, isoproterenol, or an NO donor were inhibited. Consistently, Mihm et al. (879) have demonstrated that the preincubation of rat thoracic aorta segments with clinically relevant concentrations of 3-nitrotyrosine observed in various pathophysiological states resulted in concentration-dependent impairment of endotheliumdependent vascular relaxation, and induced DNA damage in vascular endothelial cells. They also found a highly statistically significant association between 3-nitrotyrosine-induced endothelial dysfunction and frequency of TUNEL-positive cells, suggesting that DNA fragmentation and/or apoptosis may be an important mechanism of endothelial dysfunction in vivo, and that 3-nitrotyrosine may participate in the initiation of these events $(879,1473)$. Peroxynitrite was also reported to inhibit voltage-gated $\mathrm{K}^{+} \mathrm{K}(\mathrm{v})$ and $\mathrm{Ca}^{2+}$-activated $\mathrm{K}^{+}$ channels in coronary arterioles $(754,786)$ and vascular prostacycline synthase $(1466,1467$, 1472,1475). Repetitive exposure to a nominal concentration of $100 \mu \mathrm{M}$ peroxynitrite, or greater, resulted first in a marked vasodilatation in Langendorff heart preparations, isolated 
coronary, pulmonary, and cerebral arteries presumably by mechanisms including the secondary formation of molecules with the properties of NO donors (e.g., nitrosothiols), and by activation of ATP-dependent $\mathrm{K}^{+}$channels $(744,783,909,1328,1365,1389)$. However, these experiments showed tachyphylaxis, since vasodilation could no longer be elicited after repetitive exposures to these large amounts of peroxynitrite (1136) and loss of ATP-dependent $\mathrm{K}^{+}$channel function (471).

The systemic application of peroxynitrite in anesthetized rats also elicited an initial pronounced fall in mean arterial blood pressure followed by a rapidly developing tachyphylaxis and increase in blood pressure $(83,84,471-473,704)$. It is likely that these responses are due to the effects of peroxynitrite, or to the resulting vasoactive reaction products on the vasculature $(470,1328)$. Intravenous administration of 3-nitrotyrosine, to anesthetized rats markedly inhibited the hemodynamic responses produced by catecholamines (norepinephrine and epinephrine), $\alpha 1$-adrenoceptor agonist phenylephrine, $\beta$-adrenoceptor agonist isoproterenol, as well as angiotensin II, raising the possibility that 3-nitrotyrosine may be involved in the pathogenesis of hemodynamic alterations associated with inflammatory conditions, such as I/ $\mathrm{R}$, myocarditis, chronic heart failure, atherosclerosis, and sepsis, where formation of peroxynitrite is favored $(705,706)$. Tables $1-3$ summarize the known targets of peroxynitriteinduced protein nitration that might be relevant to the majority of cardiovascular pathophysiologies discussed in the following parts.

2. Myocardial I/R injury-Reperfusion injury is the leading cause of tissue damage occurring in conditions such as myocardial infarction, stroke, organ transplantation, and cardiopulmonary bypass, as well as a major mechanism of end-organ damage complicating the course of circulatory shock of various etiologies. In all these conditions, the initial trigger of the damage is the transient disruption of the normal blood supply to target organs followed by reperfusion. From a clinical viewpoint, no therapy is currently available to limit reperfusion injury, which emphasizes the importance of a better understanding of its underlying pathological mechanisms, to devise potential future therapeutic strategies.

Reperfusion of ischemic myocardium is the ultimate treatment to reduce myocardial damage. Unfortunately, reperfusion itself leads to additional tissue injury mediated by numerous factors including reactive oxygen (superoxide anion, hydrogen peroxide, and hydroxyl radical) and reactive nitrogen species (e.g., peroxynitrite and nitrogen dioxide) upon reperfusion (reviewed in Refs. 386,995,1083), as well as to the rapid transcriptional activation of an array of proinflammatory genes $(128,197,541,642,1032)$. Immediate consequences are the local sequestration and activation of polymorphonuclear leukocytes, resulting in a rapid amplification of the initial inflammatory response and ROS generation, the so-called "respiratory burst" $(799,800)$. Reactive oxygen species in I/R can also be produced by additional sources such as mitochondria, NAD(P)H oxidases, cyclooxygenase, NOS, and xanthine oxidase (reviewed in Refs. 478,993,995,1306). Indeed, since the introduction of the concept of I/R injury in the early 1980s (467,858), several lines of evidence support the pathophysiological role of xanthine oxidase-derived ROS generation, and the beneficial effects of xanthine oxidase inhibitors such as allopurinol and oxypurinol against myocardial I/R injury (993). The burst of ROS immediately upon reperfusion initiates a chain of deleterious cellular responses eventually leading to coronary endothelial dysfunction (1292); adherence of neutrophils to endothelium, transendothelial migration, and the release of mediators (600, 743); transient impairment of left ventricular systolic contractile function or "myocardial stunning" $(113,131)$; acute diastolic dysfunction; cellular calcium overload $(112,113)$; reenergization-induced myocyte hypercontracture (1030); arrhythmia; and cell death.

The first evidence implicating that increased peroxynitrite formation during myocardial $\mathrm{I} / \mathrm{R}$ might contribute to the tissue damage came from the study by Wang and Zweier (1344). They 
used isolated rat hearts subjected to global ischemia to measure the release of NO, superoxide, and peroxynitrite from the coronary effluent utilizing EPR spectroscopy and luminol chemiluminescence. The release of NO, superoxide, and peroxynitrite was increased within the first 2 min of reperfusion, and postischemic contractile function was improved by NOS inhibitors $\left[N^{\mathrm{G}}\right.$-nitro-L-arginine methyl ester (L-NAME), $N^{\mathrm{G}}$-monomethyl-L-arginine (LNMMA)], SOD, urate, or glutathione $(212,429,1344,1478)$. The likelihood of peroxynitritemediated injury was further supported by the demonstration of increased nitrotyrosine immunostaining both in hearts perfused with peroxynitrite or subjected to I/R, but not in controls (1344). A similar observation was reported by Yasmin et al. (1413) using dityrosine fluorescence as an index of peroxynitrite generation upon reperfusion of buffer-perfused rat hearts subjected to ischemia. Consistently, peroxynitrite aggravates myocardial reperfusion injury in isolated perfused rat heart (816) and contributes to the development of reperfusion arrhythmias (1268). The marked inhibition of both contractility and respiration during reoxygenation of isolated rat left ventricular papillary muscles or hearts prior subjected to hypoxia could be attenuated by SOD or urate, suggesting that the formation of peroxynitrite contributes to the suppression of cardiac contractile performance and mitochondrial respiration caused by hypoxia and reoxygenation $(740,1380,1396)$. The peroxynitrite-induced tyrosine nitration of prostacyclin synthase was implied to be responsible for the hypoxia/reoxygenationinduced coronary vasospasm in isolated bovine coronary arteries $(1466,1467,1472,1475)$. Chronic treatment of rats with SOD mimetic EUK-8 or estrogen markedly improved functional recovery of isolated perfused hearts subjected to $I / R$, which was associated with decreased NADPH oxidase expression and myocardial nitrotyrosine staining (1398). Interestingly, the most commonly used pain medication, acetaminophen, either administered to the perfusion buffer or used as a chronic treatment was found to attenuate the myocardial damage and peroxynitrite formation induced by myocardial I/R in isolated perfused rat hearts $(453,869$ 871,1398).

Liu et al. (781) using an in vivo rat model of regional myocardial I/R provided immunohistochemical evidence of increased myocardial iNOS expression and protein nitration. These results are also supported by an in vivo dog study demonstrating that intracoronary administration of L-arginine (the precursor of NO) aggravates myocardial stunning (prolonged contractile dysfunction during reperfusion following a brief episode of myocardial ischemia) and increases nitrotyrosine immunoreactivity in the myocardium (902). Consistent with this observation, NOS inhibition decreases coronary sinus free radical concentration, myocardial nitrotyrosine formation, and ameliorates myocardial stunning following I/R in a similar in vivo canine model, while NO donor SNAP exerts opposite effects $(1437,1438)$. Another recent study has suggested that peroxynitrite-induced myocardial protein nitration contributes to the enhanced stunning and development of infarct in pigs with regional cardiac denervation (573). Recent in vivo studies have also provided convincing evidence that various potent cell-permeable metalloporphyrin-based peroxynitrite decomposition catalysts reduce myocardial infarct size, myocardial protein nitration, and improve cardiac function in rat and pig models of myocardial I/R (100,750,985,1027) (Fig. 10).

Intriguingly, several recent studies have investigated the role of peroxynitrite in myocardial I/ $\mathrm{R}$ in human patients undergoing open heart surgery $(525,526,864)$. In these studies, plasma nitrotyrosine levels from coronary sinus effluent and/or arterial blood $(525,526)$ or myocardial nitrotyrosine immunoreactivity (864) from left ventricular biopsy specimens were evaluated before and at the end of cardiopulmonary bypass. The difference between plasma nitrotyrosine levels from coronary sinus effluent and arterial blood (index of myocardium-derived peroxynitrite generation) peaked at $5 \mathrm{~min}$ following reperfusion, and was significantly correlated with the peak coronary sinus effluent and arterial blood difference in plasma malondialdehyde concentrations (index of myocardial oxidative stress and lipid peroxidation), and with postoperative maximum creatinine kinase level (index of myocardial damage) 
(526). Furthermore, the cardioplegia-induced myocardial I/R was also associated with increased iNOS expression, nitrotyrosine, and 9-isoprostane formation in human myocardium (864), indicating that the injury was likely to be mediated by both peroxynitrite and ROS. Increased immunostaining for iNOS and nitrotyrosine was detected in left ventricular biopsy specimens of patients with hibernating myocardium, a state of chronic contractile dysfunction present at rest in a territory subtended by a stenosed coronary artery that recovers following revascularization, most likely originated from repetitive episodes of transient ischemia (47). Increased iNOS expression and nitrotyrosine formation were also demonstrated in human coronary arteries of patients with human transplant coronary artery disease, a major cause of late mortality after cardiac transplantation (1069), and during cardiac allograft rejection (1249).

Interestingly, serum urate levels are elevated following myocardial I/R in experimental animals (1271) and also in patients undergoing thrombolysis and percutaneous transluminal coronary angioplasty (1412). Because urate is a well-known natural inhibitor of peroxynitrite-dependent nitration, these findings raise the intriguing possibility that increased urate production during hypoxia may represent an endogenous protective mechanism against peroxynitrite-mediated damage (1271).

In contrast to the large number of studies discussed above demonstrating detrimental effects of exogenous and/or endogenous peroxynitrite or nitrotyrosine during myocardial I/R, there are only a few reports describing beneficial (e.g., reduced infarct size, improved contractile and vascular function) or biphasic effects when peroxynitrite is injected into the blood, but not into the crystalloid environment at the time of reperfusion $(744,950,951,1084)$. However, the actual concentration of peroxynitrite delivered to its cellular targets in these experiments is questionable since at physiological $\mathrm{pH}$ the molecule undergoes rapid reactions and transformations with certain blood and nearby tissue components such as carbon dioxide, NO, thiol-containing albumin, glutathione, and cysteine, among many others (613). The oxidization of thiols by peroxynitrite leads to formation of NO donor nitrosothiols, which have been reported to protect the heart during $\mathrm{I} / \mathrm{R}$, in addition to the neutralization of peroxynitrite itself $(211,854,1328)$. It should also be noted that to our knowledge, there are no published studies demonstrating a tissue-protective effect of endogenously formed peroxynitrite. On the contrary, multiple lines of evidence discussed above and below support the toxicity of endogenously formed peroxynitrite in myocardial I/R and various other cardiovascular pathologies (reviewed in Refs. 995,1300; Table 4).

\section{Preconditioning, postconditioning, and development of nitrate tolerance-}

Ischemic preconditioning (IPC), a potent endogenous form of cardioprotection against I/R injury, was first introduced by Murry et al. in 1986 (920). In hearts subjected to I/R IPC, brief episode(s) of ischemia applied before I/R reduces infarct size as well as the incidence and severity of postischemic arrhythmias and enhances the recovery of cardiac and vascular function (reviewed in Ref. 1415). Postconditioning is a recently described form of cardioprotection (similar to preconditioning in degree of protection) when a short series of repetitive cycles of brief reperfusion and reocclusion of the coronary artery are applied immediately at the onset of reperfusion $(1415,1449)$. Numerous studies have suggested that NO, superoxide, and peroxynitrite, among many other mediators, may also be involved in the mechanisms of this endogenous protection (reviewed in Refs. 87,386,636,1149). Most notably, while endogenous peroxynitrite formation may contribute to the endogenous IPC-triggered protection, the preconditioning and the postconditioning by itself reduces both peroxynitrite/ nitrotyrosine formation and the activation of the necrosis-inducing enzyme PARP upon subsequent I/R, leading to attenuated myocardial damage $(14,252,770,1341)$. Similarly, as already mentioned above, chemical preconditioning with various drugs $(100,453,750,869-$ $871,985,1027,1297,1398)$ also leads to reduced formation of nitrotyrosine in the myocardium. 
Most studies above also support an important role of peroxynitrite-induced protein tyrosine nitration in the development of postischemic myocardial injury.

For more than a century, nitroglycerin and other organic nitrates have been effectively used to treat acute myocardial infarction, various anginas, and congestive heart failure (1272).

However, during chronic treatment, the efficacy of nitrates is often lost secondary to the development of nitrate tolerance. Numerous recent studies have suggested that the redox modification of key enzymes of the NO-soluble guanylate cyclase (sGC)-cGMP-cGMPdependent protein kinase (cGK) pathway and PDE (the enzyme responsible for the cGMP catabolism) might explain the partial decrease in the response to endothelium-dependent and other NO-dependent vasodilators, giving rise to the oxidative stress concept of nitrate tolerance $(283,461)$. Indeed, several studies have demonstrated that superoxide or peroxynitrite may inhibit sGC, presumably through oxidation of thiol groups in its catalytic site that appear to be critical for the direct activation of the enzyme by nitroglycerin $(32,650,1351)$. Furthermore, peroxynitrite and superoxide are known to impair the activity of ion channels responsible for the generation of $\mathrm{K}^{+}$and $\mathrm{Ca}^{2+}$ currents, the final mediators of nitroglycerin-induced vasodilatation $(482,785)$. Also, as mentioned in section $v B 5$, activation of $\mathrm{PKC}$ in endothelial cells by peroxynitrite has been proposed as an additional mechanism of nitrate tolerance.

\section{Myocarditis, transplant coronary artery disease, and cardiac allograft} rejection-Acute viral myocarditis accounts for the majority of "idiopathic" dilated cardiomyopathies and is the leading cause of cardiac failure in young patients. The clinical course is often unpredictable with limited cardiac dysfunction and inflammation. On the other hand, in a subset of patients, it may be associated with overwhelming inflammation leading to fulminant acute cardiac injury or chronic heart failure due to autoimmune myocarditis with very limited treatment options.

The role of NO in myocardial inflammatory disease is controversial. Some investigators have suggested that iNOS overexpression in the hearts (found predominantly in the infiltrating inflammatory cells) in experimental mouse models of viral Coxsackie B3 myocarditis is beneficial (presumably by the antiviral effect of NO) $(797,884)$, which is consistent with the more severe myocarditis in response to viral infection in iNOS knockout mice $(825,1352)$.

In contrast, in a more recent study increased levels of NO as a result of iNOS overexpression correlated with increased numbers of heart lesions and increased myocardial nitrotyrosine accumulation in infected mice (96). Similarly, progressive cardiac dysfunction correlated with increased myocardial inflammation and protein nitration in a murine model of AIDS-induced myocarditis (201). Most importantly, increased nitrotyrosine immunoreactivity was found in human biopsy specimens with viral myocarditis and sepsis, suggesting a pathogenetic role of peroxynitrite formation and/or protein nitration in these diseases (201,707; Fig. 11). Consistent with these later observations, mixture of inflammatory cytokines or endotoxin induces cardiac dysfunction, increased iNOS expression, superoxide and peroxynitrite generation, and MMP-2 activation in myocytes or isolated-perfused mouse or rat hearts, and these changes were attenuated by iNOS inhibitor or peroxynitrite scavenger $(384,428,604,663,670,729)$. Increased production of $\mathrm{NO}$ and superoxide and nitrotyrosine staining were also observed in a rat model of autoimmune myocarditis induced by porcine myosin $(616,1160)$ and in dog hearts injected with intracoronary inflammatory cytokines $(210,978)$.

Increased iNOS expression and nitrotyrosine formation were also found in human coronary arteries of patients with human transplant coronary artery disease (1069) and during cardiac allograft rejection both in experimental models $(11,1104,1247)$ and in human hearts $(1069$, $1249,1371)$. Furthermore, iNOS gene deletion or treatment with an iNOS inhibitor or metalloporphyrin-based peroxynitrite decomposition catalysts improved graft function, 
decreased histological rejection, and attenuated myocardial lipid peroxidation, nitrotyrosine formation, and PARP activation in mouse and rat cardiac transplant models $(342,1027)$. Collectively, the above-mentioned results strongly suggest that NO, superoxide, and peroxynitrite contribute to tissue injury in cardiac allografts during acute rejection (see also Table 4).

5. Chronic heart failure-Chronic heart failure (CHF) represents a major and growing public health concern that affects all Western countries and is the leading cause of hospitalization, morbidity, and mortality worldwide. CHF is considered a multiorgan and multicell syndrome characterized by myocardial functional and structural abnormalities, sodium retention, neurohumoral maladaptations, and vascular dysfunction. Different pathophysiological conditions, such as acute and chronic ischemic heart disease resulting from impaired coronary artery circulation or infarction, pressure overload, myocarditis, cardiomyopathies, and defects in genes encoding contractile apparatus, cytoskeleton, mitochondrial proteins, or intercellular matrix ultimately bring about impaired myocardial function (reviewed in Refs. 516,905,995,1306;Fig. 12). The complex interplay of diverse secondary pathways (neuropeptides, neurohormones, cytokines, iNOS, oxidative stress and peroxynitrite, MMPs, and the nuclear enzyme PARP) is involved in the deleterious way of the progression of cardiovascular dysfunction to heart failure, resulting in abnormalities in various cardiac receptors and signaling processes, calcium homeostasis, contractile proteins, besides structural alterations such as cardiovascular remodeling with hypertrophy, fibrosis, necrosis, and cardiac dilation (reviewed in Refs. 516,905,995,1306; Figs. 9 and 12). The increased peripheral resistance (a result of vasoconstriction and vascular remodeling) coupled with adverse cardiac remodeling further exacerbate heart failure initiating a vicious self-destructive cycle (Fig. 12).

Numerous experimental and clinical studies have demonstrated increased production of reactive oxygen species (ROS: superoxide, hydrogen peroxide, hydroxyl radical) both in animals and patients with CHF (reviewed in Ref. 1306). Myocardial ROS generation has been shown to be triggered by repetitive episodes of $\mathrm{I} / \mathrm{R}$, increased levels of inflammatory cytokines (e.g., TNF- $\alpha$, IL-6), impaired antioxidant defense mechanisms, and catecholamine autooxidation and/or during prostaglandin biosynthesis. The possible sources of increased production of superoxide, hydrogen peroxide, and hydroxyl radical in failing myocardium are multiple, including xanthine oxidase, $\mathrm{NAD}(\mathrm{P}) \mathrm{H}$ oxidases, cyclooxygenases, the mitochondrial electron transport chain activity, activated neutrophils, NO synthases, and auto-oxidation of certain tissue metabolites (reviewed in Refs. 993,1182,1306;Fig. 9). It is particularly exciting that xanthine oxidase inhibitors allopurinol and its active metabolite oxypurinol, the most widely used drugs for the clinical management of gout and conditions associated with hyperuricemia, show considerable promise in the treatment of CHF not only in experimental animals but also in small-scale human clinical trials (993).

Although numerous experimental and human studies have demonstrated overexpression and increased activity of iNOS in the myocardium of animals and patients with various forms of heart failure and benefits of iNOS inhibition on cardiac function $(208,383,389,421,529,693$, $878,1317,1409,1465$; Table 4), the role of iNOS and NO in the development and progression of the heart failure is a subject of recent debate (914). For example, one mouse study found positive correlation between the chronic overexpression of iNOS and peroxynitrite generation with cardiac enlargement, conduction defects, sudden cardiac death, and less commonly heart failure in mice (913), but this was not confirmed by another study (534). Increased myocardial iNOS activity was found to be responsible for depressed myocardial contractility and betaadrenergic hyporesponsiveness in rats with volume-overload heart failure (439), but iNOS deficiency did not attenuate CHF in mice (637). Additional sources of increased NO production in the failing heart could be nNOS (286) and eNOS (281). In heart failure, the cGMP pathway 
is also disrupted, either with impaired production of $\mathrm{NO}$ or its excessive degradation or neutralization with oxidants such as superoxide, thus impairing the soluble guanylate cyclase signaling pathway $(372,995)$.

The increased superoxide production and increased NO from iNOS overexpression favor the generation of peroxynitrite. As already mentioned, peroxynitrite generation has been demonstrated in various forms of myocardial I/R and myocarditis both in experimental animals and biopsies obtained from human subjects (995,1300;Fig. 10,Table 4). Increased myocardial iNOS expression, nitrotyrosine formation, and/or MMP-2 activation have also been reported in acute and chronic mouse models of doxorubicin-induced heart failure $(44,883,985$, 1355 ; Fig. 13), in heart failure induced by permanent left anterior coronary artery ligation in mice (383) and rats $(878,987)$, or by pacing in dogs (192). Importantly, increased myocardial iNOS expression and nitrotyrosine formation correlated with deterioration of cardiac function $(883,1355)$ in mice. A correlation between increased nitrotyrosine formation with myofibrillar creatine kinase inhibition was also observed in human atrial appendages from patients with fibrillation (881). Increased iNOS protein expression also correlated with increased nitrotyrosine formation in myocardial specimens of patients with heart failure. Although iNOSpositive patients were generally characterized by larger left ventricular volume and depressed function, the preserved generation of NO appeared to be associated with higher cardiac work due to the preserved Frank-Starling relationship in end-stage heart failure (1317). Increased nitration of SERCA2a in idiopathic dilated cardiomyopathic human hearts was also reported, and a positive correlation between the time to half relaxation and the nitrotyrosine/SERCA2a content in myocytes was observed (789). Furthermore, a novel peroxynitrite decomposition catalyst attenuated the development of cardiac dysfunction and myocardial nitrotyrosine formation, and increased the survival in doxorubicin-induced cardiomyopathy model (985).

It appears that peroxynitrite decomposition catalysts exert beneficial effects on cardiac and endothelial function in various models of myocardial injury (e.g., heart failure, myocardial I/ $\mathrm{R}$, myocarditis, cardiac allograft rejection, and diabetic cardiomyopathy; see also below). The mechanism by which peroxynitrite neutralization protects hearts from dysfunction may involve protection against vascular and myocardial tyrosine nitration, lipid peroxidation, and inactivation of contractile proteins, MMP and PARP activation, and multiple other mechanisms shown in Figure 9 and listed in Table 3. These observations support the concept that peroxynitrite is a major mediator of myocardial injury in various pathological conditions, and its effective neutralization or inhibition of downstream effector pathways (e.g., PARP and MMP activation) can be of significant therapeutic benefit $(624,986,987,989,995,1234,1246)$.

\section{B. Nitric Oxide and Peroxynitrite in Vascular Diseases}

Endothelial cells in response to various physical and chemical stimuli (e.g., shear stress, change in pressure and $\mathrm{pH}$ ), circulating hormones, cytokines, drugs, and substances released by sensory and autonomic nerves or platelets produce vasoactive relaxing substances (e.g., NO, endothelium-derived hyperpolarizing factor, prostacyclin, adenosine, $\mathrm{C}$-natriuretic peptide) and contracting substances (e.g., angiotensin II, endothelin-1, thromboxane $\mathrm{A}_{2}$, isoprostanes) that regulate vascular tone and permeability, hemostasis, angiogenesis, and inflammation (381). The vascular endothelium sustains the balance between prevention and stimulation of platelet aggregation, thrombogenesis and fibrinolysis, promotion and inhibition of the smooth muscle cell proliferation and migration, and also between vasoconstriction and vasodilation (289). The disruption of this tightly controlled balance leads to the development of endothelial dysfunction, a multifaceted disorder, which represents a predominant early feature of diabetes, hypertension, heart failure, and atherosclerosis and makes this population prone to cardiovascular complications and microthrombus formation (166,381,731). Although the hallmark of endothelial dysfunction is impairment of the endothelium-dependent vasodilation, 
other alterations (e.g., inflammation, increased lipoprotein oxidation, vascular smooth muscle proliferation and migration from the media to the intima, extracellular matrix deposition or lysis, platelet activation, and thrombus formation) associated with this disorder have also been described (365). Accumulating evidence supports the view that the endothelial dysfunction associated with diabetes, hypertension, heart failure, and atherosclerosis is related to the local formation of reactive oxygen and nitrogen species in the vicinity of the vascular endothelium (reviewed in Refs. 365,478,630,757,775,996,1004,1306,1378,1380,1381).

As already mentioned previously and summarized in Table 3, peroxynitrite may contribute to vascular pathophysiology by various mechanisms including triggering apoptosis and/or PARPdependent cell death in endothelial $(319,879)$ and vascular smooth muscle $(755,756)$ cells, inducing upregulation of adhesion molecules in endothelial cells, endothelial glycocalyx disruption, enhancing neutrophils adhesion $(424,723,1178,1447,1477)$, inhibiting voltagegated $\mathrm{K}^{+} \mathrm{K}(\mathrm{v})$ and $\mathrm{Ca}^{2+}$-activated $\mathrm{K}^{+}$channels in coronary arterioles $(754,786)$ and vascular prostacyclin synthase $(228,290,1466,1467,1472,1475)$, and dependent on the environment stimulating or inhibiting platelet aggregation $(908,953)$. Additionally, peroxynitrite-mediated oxidation of tetrahydrobiopterin, a critical cofactor for NO synthase, may represent a pathogenic cause of uncoupling of $\mathrm{NO}$ synthase, leading to production of superoxide rather than NO (405-407,734). Furthermore, clinically relevant concentrations of 3-nitrotyrosine (present in various pathophysiological diseases, Table 4) result in concentration-dependent impairment of acetylcholine-induced, endothelium-dependent vascular relaxation and induce DNA damage in vascular endothelial cells $(879,1473)$.

Below, we are focusing on the evidence implicating peroxynitrite in the pathophysiology of vascular dysfunction associated with atherosclerosis, restenosis, hypertension, aging, and hyperhomocysteinemia. The cardiovascular dysfunction associated with heart failure, shock, and diabetes is reviewed in later or earlier parts (see also Tables 4 and 5).

1. Atherosclerosis and restenosis-Atherosclerosis is the principal cause of common cardiovascular disorders such as coronary artery disease, various forms of heart failure and stroke, abdominal aortic aneurysms, and ischemic gangrene, which are the leading causes of death in the Western society. The prevalence of atherosclerosis is increasing worldwide in all age groups including the adolescent population (1207). The pathophysiology of atherosclerosis is multifaceted. It comprises endothelial injury leading to accumulation of lipids and their uptake by monocytes that is followed by platelet and monocyte adhesion and aggregation at the site of injury, and the release of a variety of factors that promote smooth muscle migration and proliferation (from the media into the intima), which then synthesize and deposit extracellular matrix $(514,603)$.

Percutaneous transluminal angioplasty (surgical procedures aimed to repair the stenotic blood vessel by inflating a balloon-tipped catheter at the site of the vascular narrowing) is performed in $\sim 2$ million people worldwide annually and has become a widely available and efficient treatment option for patients with peripheral and coronary artery disorders. In many of these cases, a stent (an expandable wire mesh or hollow perforated tube) is inserted into the reconstructed blood vessel, to provide support after angioplasty and protection against restenosis. Despite the significant advances made in these surgical procedures during the past decade, the restenosis remains a significant clinical issue with a limited number of therapeutic options, affecting $20-30 \%$ of patients undergoing vascular interventions.

Recent experimental, clinical, and epidemiological studies have revealed the importance of the cross-talk between inflammation, generation of reactive oxygen and nitrogen species, and lipid metabolism in the pathogenesis of atherosclerosis and vascular remodeling following injury $(37,514,518,931,1012,1090,1093)$. There is also evidence suggesting that atherosclerosis is 
not only associated with decreased NO bioavailability, but also with alterations in signaltransduction components downstream of NO, including among others, the NO receptor sGC, particularly in neointima $(372,865)$.

Numerous studies support both anti- and proatherogenic roles of NO $(80,164,183,203,229$, $524,561,734,742,932,963 \mathrm{a}, 1364)$ and are covered by numerous excellent overviews $(514$, $931,1012,1090,1093)$. One explanation for this paradox is that NO alone is an important antiatherosclerotic autacoid with antiaggregatory effects on platelets, antioxidant, antiinflammatory, antiproliferative, and vasodilatory effects on vasculature, while in combination with proinflammatory oxidants (e.g., superoxide, hydrogen peroxide, hypochlorite) it forms proatherogenic mediators (e.g., peroxynitite) that modify lipids and proteins $(78,899,1009$, $1010,1091,1093,1300,1314)$. According to the classic view, reactive oxygen and nitrogen species oxidatively damage LDL trapped in the arterial intima forming oxidized LDL, which in turn initiates numerous events (e.g., foam cell formation, monocyte recruitment and adhesion to the endothelium, inhibition of macrophage motility, smooth muscle cell proliferation, promotion of cytotoxicity, and attenuation of vascular reactivity) facilitating the development of atherosclerotic lesions (1012). Supporting the pathogenetic role of peroxynitrite in atherosclerosis, numerous studies have demonstrated increased 3-nitrotyrosine and iNOS expression in human atherosclerotic tissue $(49,80,164,240,313,363,584,742,802,1019,1211)$, which correlated with plaque instability in patients $(313,584)$. Peroxynitrite most likely contributes to the development of early vascular lesions and as the lesion develops, other reactive nitrogen species derived from the reaction of nitrite with proteins, such as myeloperoxidase, in inflammatory cells, are also thought to contribute to nitrosative stress $(530,835,1012,1033)$. Numerous studies have also demonstrated that peroxynitrite is able to modify proteins and lipids in LDL and high-density lipoprotein (HDL) even in the presence of endogenous lipophilic antioxidants $(287,465,558,899,1002,1009,1010,1019,1094)$ and regulates signaling pathways in the endothelial and vascular smooth muscle, thereby modulating the vascular response to atherogenetic stimuli $(450,1093,1221)$. Importantly, various drugs (e.g., cicletanine and estradiol) and fasting, which improve endothelial dysfunction or decrease the atherosclerosis progression in animal models of disease, also reduce serum or vascular nitrotyrosine level/staining $(653,1250,1411)$, further supporting the pathogenetic role of peroxynitrite in atherosclerosis.

Recent studies also demonstrate enhanced production of superoxide and peroxynitrite in hypercholesterolemia, hyperlipidemia, and hyperhomocysteinemia, which are considered to be major risk factors for the development of atherosclerosis $(42,70,339,343,579,635,912$, $1181,1202,1264,1303,1431,1435)$.

There is also accumulating evidence demonstrating that reactive oxygen and nitrogen species and downstream effector pathways (e.g., PARP) play an important role in the pathogenesis of restenosis following vascular injury $(37,82,618,667,747,921)$. Numerous studies found increased 3-nitrotyrosine immunoreactivity and/or iNOS overexpression in media and neointima following balloon injury (a model of restenosis) and increased 3-nitrotyrosine-totyrosine ratio in the serum of patients following stent implantation $(37,82,602,618,667,747$, 921). The serum 3-nitrotyrosine-to-tyrosine ratio appears to be an independent predictor of angiographic late lumen loss in patients (602). Supporting the pathogenetic role of superoxide and peroxynitrite and downstream effector pathways in restenosis, administration of SOD, SOD mimetic, NAD(P)H oxidase, or PARP inhibitors decreased not only vascular remodeling following injury but also 3-nitrotyrosine formation $(82,618,747,921)$. In contrast, in experimental models of restenosis, systemic administration of $\mathrm{NO}$ donors $(846,1144)$ or local

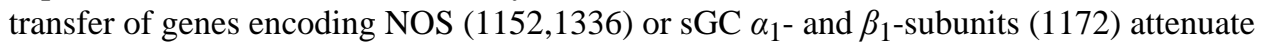
neointima formation. 
2. Aging-Epidemiological studies revealed that even in the absence of established risk factors such as hypertension, diabetes, and hyperhomocysteinemia, advanced age itself significantly increases cardiovascular morbidity $(726,727,927)$. Although there is substantial evidence demonstrating an aging-associated development of cardiac and vascular dysfunction $(25,26,114,171,510,521,545,990,1253,1311$; reviewed in Refs. 24,726,727), the mechanisms responsible for this phenomenon have not yet been clearly established. This impairment is, at least in part, related to the increased local formation of reactive oxygen and nitrogen species coupled with impaired NO bioavailability, and associated chronic inflammation in the myocardium and vasculature $(81,249,517,601,938,1081,1212,1311$; reviewed in Refs. 51, 248,394).

Increased ROS production was reported in aorta $(509,1311)$, carotid $(509,1311)$, mesenteric (409), and small coronary arteries (249) of aged rats and mice (409). Aging-induced vascular oxidative stress is associated with increased activity of $\mathrm{NAD}(\mathrm{P}) \mathrm{H}$ oxidases $(7,249,1311)$ and/ or other oxidase mechanisms (40), a downregulation of antioxidants, such as ecSOD (1213), and an increased expression of iNOS $(409,1409)$. Increased oxidative stress in aging also leads to functional inactivation of $\mathrm{NO}$ by elevated concentrations of superoxide favoring an enhanced peroxynitrite formation $(7,249,409,1213,1311)$. Notably, age-related decline in eNOS expression $(249,556,853,1262,1386)$ and/or a decreased intracellular L-arginine availability (88) further aggravate impaired NO bioavailability, limiting cardiac blood supply, altering myocardial $\mathrm{O}_{2}$ consumption and cardiac contractility (7), and enhancing apoptosis of endothelial cells $(251,556)$.

Several studies found increased cardiovascular peroxynitrite formation and/or protein nitration in aging $(7,249,409,1213,1311)$. For example, Van der Loo et al. (1311) found increased accumulation of 3-nitrotyrosine (3-NT) in the aortas of aging rats compared with young controls; one of the nitrated proteins identified was mitochondrial Mn-SOD, a major antioxidant enzyme, which loses its activity upon nitration by peroxynitrite $(830,1405)$. Using proteomics techniques, Kanski et al. (655) identified, among others, numerous enzymes of the glycolytic machinery [ $\alpha$-enolase-1, $\alpha$-aldolase, and glyceraldehyde-3-phosphate dehydrogenase (GAPDH)] as targets for protein nitration (654). Several nitrated mitochondrial proteins (e.g., aconitase, creatine kinase, VDAC, ATP synthase, and other proteins involved in electron transfer) (654) were also discovered from the aging myocardium, suggesting that these proteins are particularly vulnerable to aging-related nitration. A more recent study investigated peroxynitrite- and 4-hydroxy-2-nonenal (HNE)-modified serum proteins from young and aging Fisher rats (676). This study identified 16 proteins (e.g., involved in blood coagulation, lipid transport, blood pressure regulation, and protease inhibition) modified by nitration and/or HNE adduction during aging (676). Another example of specific protein nitration associated with aging is the nitration of skeletal muscle and myocardial sarcoplasmic reticular $\mathrm{Ca}^{2+}$-ATPases $(692,1131,1329,1330,1397)$. Recent studies have also demonstrated that increased levels of ROS and reactive nitrogen species (RNS) may activate $\mathrm{NF} \kappa \mathrm{B}$, a redoxsensitive transcription factor involved in the induction of the transcription of a large range of genes implicated in inflammation, including cytokines (e.g., TNF- $\alpha$, IL-6, and IL-1 $\beta$ ), chemokines, and adhesion molecules $(771,1270,1441)$ expressed by both endothelial and smooth muscle cells. In agreement with this, there is recent evidence for a proinflammatory shift in cardiac (737) and vascular (249-251) cytokine expression profile (including an upregulation of TNF- $\alpha$ and IL-6) associated with aging. As discussed in previous sections in detail (see also Tables 1-3), there are numerous possible additional downstream targets of peroxynitrite-induced protein nitration and cytotoxicity (e.g., antioxidant enzymes, contractile proteins, stress kinases, MMPs, PARP, etc.) that may likely play an important role in the development of aging-associated cardiovascular pathophysiology $(991,992,998$; reviewed in Refs. 248,1227). 
3. Hypertension-Arterial hypertension, the chronic elevation of blood pressure, is a major public health concern even though it is not a disease per se. Untreated hypertension leads to premature morbidity and mortality due to stroke, accelerated coronary arterial disease, myocardial infarction, and cardiac and renal failure. In the majority of cases, the principal cause of the disease remains elusive (primary, essential, or idiopathic hypertension), and therapy, therefore, is symptomatic rather than preventive (506). The vascular effects of the chronic elevation of blood pressure are complex and comprise both local effects on the vascular endothelium and smooth muscle and effects mediated by the CNS and increased production of various neurohumoral factors (e.g., angiotensin II, catecholamines, endothelin, etc.) (499, 698,1035).

Accumulating evidence suggests that alterations in NO synthesis and NO-sGC-cGMP signaling or a reduction in the bioavailability of endothelium-derived NO by increased oxidative stress are key contributors to the pathogenesis of hypertension (reviewed in Refs. $365,372,698,964,1004,1379)$. Numerous experimental studies using animal models of disease [e.g., angiotensin II infusion, deoxycorticosterone with a high salt diet (DOCA salt), Dahl hypertensive rats, spontaneously hypertensive rats (SHR), chronic inhibition of eNOS, and aortic banding] and clinical reports have established that hypertension is associated with increased superoxide production, dysregulation of NOS, and endothelial dysfunction. However, the exact molecular mechanisms of these processes are not fully understood (95, $166,167,172,269,416,535,699,733,889,1065,1180,1305,1335,1343)$.

There is controversy as to whether the endothelial dysfunction in hypertension is the cause or a consequence of increased blood pressure (1318). Likewise, the source of superoxide in hypertension is also a matter of recent debate; it may include $\mathrm{NAD}(\mathrm{P}) \mathrm{H}$ oxidases, xanthine oxidase, mitochondrial enzymes, and uncoupled NOS (reviewed in Refs. 365,993,1004, 1381,1382,1408). Nevertheless, increase in intraluminal pressure and oscillatory shear stress (both are associated with hypertension) have been shown to activate redox-sensitive signaling pathways, enhance oxidative stress in the vasculature, and impair endothelial function both in vivo and in vitro $(166,167,1004,1304,1305,1381,1408)$. Increased levels of superoxide have been shown to decrease the bioavailability of $\mathrm{NO}$, thereby contributing to the maintenance of elevated peripheral resistance $(166,167,698,699,1305)$. Production of various vasoconstrictor substances (e.g., angiotensin II, endothelin-1, thromboxane $\mathrm{A}_{2}$, isoprostanes, etc.) may also contribute to increased ROS production and reduced endothelium-dependent relaxation in hypertension $(365,1004)$. The hypertrophy of the vascular wall as a consequence of reduced NO bioavailability can further aggravate an increase in the vascular resistance $(365,1004)$.

Compelling evidence has emerged supporting the importance of endogenous peroxynitrite formation and protein nitration in the pathogenesis of arterial hypertension (reviewed in Refs. $365,1300)$. Increased superoxide formation, gene expression of several subunits of $\mathrm{NAD}(\mathrm{P}) \mathrm{H}$ oxidases, eNOS, and nitrotyrosine were markedly increased in the aorta segment above aortic coarctation (hypertensive zone) and in hearts of aortic-banded rats and mice compared with normotensive controls $(65,125,724,1324)$. Interestingly, substantial changes in endothelial dysfunction were only observed when NOS expression and superoxide production were simultaneously increased and were associated with enhanced nitrotyrosine formation (125, 1300 ), suggesting that enhanced superoxide production alone is not sufficient to produce endothelial dysfunction. Increased peroxynitrite formation or protein nitration was also reported in the serum $(643,1402)$, vasculature $(269,565,643,776,815,1217,1252)$, and kidneys (1080) of SHR rats, malignant stroke-prone spontaneously hypertensive rats (M-SHRSP), and SHR/ND-mcr-cp (SHR/cp) rats (genetic model of the metabolic syndrome). Antioxidant-rich $\operatorname{diet}$ (1080), the SOD mimetic M40403 (269), the polyphenol chlorogenic acid (1217), the angiotensin-converting enzyme inhibitor ramipril (776), the adrenoceptor blocker with antioxidant properties carvedilol (815), and the cofactor of eNOS tetrahydrobiopterin (565) 
attenuated hypertension and nitrotyrosine formation in vasculature and kidneys and improved compromised vascular function and end-organ damage.

Angiotensin II, a well-known factor in the pathogenesis of most cardiovascular disorders and a natural regulator of blood pressure, can induce superoxide formation mediated at least in part by vascular $\mathrm{NAD}(\mathrm{P}) \mathrm{H}$ oxidases $(416,478,733,889,1065)$. Reactive oxidant species in turn can exert direct oxidative effects, but can also signal through pathways such as mitogen-activated protein kinases, tyrosine kinases, and transcription factors and lead to events such as inflammation, hypertrophy, remodeling, and angiogenesis $(478,969,1004)$. Recent studies have demonstrated that angiotensin II can also induce peroxynitrite formation and PARP activation in endothelial cells in vitro $(880,1236)$ and in aorta, heart, and kidneys of rats $(492,656,1236,1349)$ and mice $(1159,1342)$ following chronic infusion in vivo. Protein nitration correlated with the extent of endothelial dysfunction (1349), and both were attenuated by supplementation with tetrahydrobiopterin (656).

Other forms of experimental hypertension induced by lead (325,1323), mineralocorticoids (1214), cyclosporin (170), diet-mediated obesity $(327,1077)$, and renovascular/renal dysfunction $(119,327,1325)$ are associated with increased ROS-mediated inactivation of NO with sequential increases of 3 -NT in plasma $(170,1323,1325)$, vasculature $(119,327,1077$, $1325)$, heart $(1077,1214,1323,1325)$, kidney $(119,327,1077,1323)$, liver $(1077,1325)$, and brain (1323). Remarkably, in most of these conditions, the decrease in blood pressure and improvement in hypertension-associated endothelial dysfunction and end-organ damage to various drugs or interventions aiming to reduce blood pressure and decrease oxidative stress (e.g., vitamin E, losartan, tempol, carvediol, diet, etc.) was always associated with reduction of 3-NT in vasculature, heart, and various other organs $(170,327,1323,1325)$. Thus it appears that peroxynitrite plays an important role in the pathophysiology of hypertension.

In conclusion, multiple lines of evidence discussed above and listed in Table 4 suggest that peroxynitrite plays an important role in various forms of cardiovascular dysfunction and injury; pharmacological neutralization of this reactive oxidant or targeting the downstream effector pathways may represent a promising strategy to treat various cardiovascular disorders.

\section{Nitric Oxide and Peroxynitrite in Circulatory Shock}

Circulatory shock defines a syndrome precipitated by a systemic derangement in perfusion leading to widespread cellular hypoxia and vital organ dysfunction. Depending on its initial pathophysiological mechanisms, shock is subdivided in three main categories, namely, cardiogenic, hemorrhagic, and septic shock. In the advanced stages, all shock states evolve to a common clinical picture characterized by profound cardiovascular failure, the activation of cellular cytotoxic effectors (polymophonuclear leukocytes), and the upregulation of an array of proinflammatory genes, leading to systemic inflammation, organ dysfunction, and death (569). An extensive body of experimental evidence supports the view that ROS and RNS, in particular peroxynitrite, are crucial components of this chain of pathophysiological events.

Circulatory shock is a perfect condition to enhance the production of peroxynitrite, as both parent radicals $\mathrm{NO}$ and $\mathrm{O}_{2}^{--}$are formed in large amounts and in close proximity in this setting $(380,766)$. The upregulated production of $\mathrm{O}_{2}{ }^{--}$principally arises from the reactions catalyzed by NADPH oxidase $(629,757)$, present in leukocytes and endothelial cells, and by the conversion of xanthine dehydrogenase into xanthine oxidase, which occurs during inflammation or episodes of tissue ischemia $(425,1261) . \mathrm{O}_{2}{ }^{--}$is also formed by the partial reduction of molecular oxygen within mitochondria, due to electron leak from the respiratory chain, a natural phenomenon that is enhanced in conditions of cellular hypoxia and oxidantmediated mitochondrial damage (947). Finally, $\mathrm{O}_{2}{ }^{--}$may be generated by the uncoupling of NOS in conditions of substrate (L-arginine) or cofactor (tetrahydrobiopterin, $\mathrm{BH} 4$ ) deficiency, 
as prevails in underperfused tissues during shock or ischemia $(1011,1392,1393)$. With respect to NO, its major sources are the enzymatic activities of the various NOS. In the early stages of shock, production of NO is essentially driven by the stimulated activity of eNOS, triggered in part by the action of platelet activating factor (PAF) on the endothelium (1226). At later stages, production of NO is markedly enhanced following the diffuse expression of iNOS, upon cellular activation by microbial products and proinflammatory cytokines (for review, see Refs. 380,766). According to some recent studies, a mitochondrial isoform of NOS (mtNOS) might also represent a significant source of NO during circulatory shock $(17,107)$. A nonenzymatic source of NO has also been described related to the reduction of nitrites in acid and reducing conditions, as can occur in ischemic tissues (833).

Formation of peroxynitrite from $\mathrm{O}_{2}{ }^{--}$and $\mathrm{NO}$ in circulatory shock has been evaluated by the detection of nitrotyrosine, and by the peroxynitrite-dependent oxidation of the fluorescent probe dihydrorhodamine 123 (DHR) to rhodamine $(605,610)$. Plasma DHR oxidation and formation of nitrotyrosine in tissues (aorta, liver, lung, and small intestine) has been detected in rodents during systemic inflammation and shock induced by zymosan, a yeast cell wall component $(257,258,261,262,355)$. These modifications were abrogated by genetic suppression of iNOS (266), and by various treatment strategies removing peroxynitrite, including MnTBAP (261), melatonin (255,355), and $N$-acetylcysteine (NAC) (257), proving the involvement of NO-derived peroxynitrite in DHR oxidation and nitrotyrosine formation. Comparable results were obtained in experiments using LPS as the inducer of shock. For example, nitrotyrosine formation occurred in skeletal muscle of rats challenged with LPS $(347,980)$, with a time course similar to that of iNOS expression in this tissue (347), as well as in the aorta $(298 \mathrm{a}, 1237)$ and in the heart, where it was suppressed by melatonin (298a), the NOS inhibitor L-NAME (1237), and the peroxynitrite decomposition catalyst FeTPPS (729). Nitrotyrosine has further been detected in the lung (12), liver (798), kidney (1430), and gut $(243,1183)$ in various animal models of septic shock. In humans, one study indicated increased plasma nitrotyrosine levels during septic shock, from undetectable levels in normal subjects to values as high as $118.0 \pm 22 \mu \mathrm{M}$ in septic patients (420). Increased myocardial nitrotyrosine immunostaining was also demonstrated from myocardial biopsy speciments of patients with sepsis (Fig. 11; Ref. 707).

Evidence also exists that peroxynitrite formation is triggered during experimental hemorrhagic shock. Szabo et al. (1238) first reported an increased plasma oxidation of DHR in rat hemorrhagic shock that could be suppressed by the NOS inhibitor L-NAME. Later studies confirmed these observations by showing the formation of nitrotyrosine in organs (lung, gut, liver, and aorta) from rats after hemorrhage and resuscitation $(767,911,1296,1460)$.

Nitrotyrosine immunostaining in hemorrhagic shock was suppressed by NOS inhibitors, by uric acid, an endogenous peroxynitrite scavenger $(1296,1460)$, and by genetic suppression of NADPH (745).

1. Mechanisms of tissue injury by peroxynitrite during circulatory shock-There are basically four major mechanisms underlying the toxicity of peroxynitrite formed from $\mathrm{NO}$ and $\mathrm{O}_{2}{ }^{-}$in shock. These include lipid peroxidation; the depletion of antioxidant reserves, especially reduced GSH; the oxidation/nitration of proteins with special emphasis on mitochondrial proteins; and finally, the induction of DNA damage leading to the activation of the nuclear enzyme PARP.

Peroxynitrite is a major initiator of lipid peroxidation, generating lipid radicals that propagate free radical reactions, thereby degrading membrane lipids $(311,560,1055)$. In vivo, the measurement of thiobarbituric acid reactive substances, such as malondialdehyde (MDA), has been used as an index of lipid peroxidation in pathological conditions. Elevated MDA levels have been measured in virtually every organ in experimental models of septic $(258,355,763$, 
$764,1109,1183)$ and hemorrhagic shock $(767,1224)$, where the contributing role of peroxynitrite has been attested by the reduction of MDA levels afforded by various antiperoxynitrite strategies including NAC (258), melatonin (355), MnTBAP, or the peroxynitrite decomposition catalysts FeTMPS and FeTMPyP (1109), as well as the NOS inhibitor and peroxynitrite scavenger mercaptoethylguanidine (MEG) (1224).

A second major mechanism of peroxynitrite-dependent toxicity in shock is its ability to rapidly oxidize GSH, the major endogenous scavenger of oxidants and free radicals $(273,709,1289)$. The importance of intact cellular GSH pools to limit peroxynitrite-induced cellular injury is illustrated by findings of Salvemini et al. (1107), who showed that endogenous GSH was essential to limit the development of vascular hyporeactivity and endothelial dysfunction in response to exogenous peroxynitrite and peroxynitrite endogenously produced during endotoxic shock. It is also noteworthy that the depletion of endogenous GSH with Lbuthionine-sulfoximine (BSO) markedly enhanced peroxynitrite-mediated tissue injury in animal models of septic shock $(273,278)$, whereas increasing cellular GSH by treatment with NAC reduced such detrimental consequences $(257,258)$.

Abnormal oxidative metabolism at the cellular level is a critical event triggering organ damage and dysfunction in shock $(393,762)$. Peroxynitrite readily inactivates mitochondrial enzymes involved in oxidative metabolism and can thereby shut down cellular energetics. This effect is particularly likely in shock, where mitochondria can produce copious amounts of peroxynitrite, due to stimulated activity of mitochondrial NOS and enhanced electron leak from the respiratory chain leading to $\mathrm{O}_{2}{ }^{--}$formation $(17,127,947,1308)$, a phenomenon further amplified by the inactivation of mitochondrial MnSOD by peroxynitrite (826). In a recent experimental study, Alvarez et al. (17) showed that, following administration of endotoxin to the rat, steady-state concentrations of peroxynitrite rose from 8 to $12 \mathrm{nM}$ in heart mitochondria and from 21 to $49 \mathrm{nM}$ in diaphragm mitochondria. The relevance of these metabolic alterations with respect to human septic shock has been highlighted in a study by Brealey et al. (132). In septic patients, a decrease in tissue ATP levels and mitochondrial complex I activity was measured in skeletal muscle biopsies, which correlated with increased clinical severity and NO production within muscles, supporting a critical role of NO-peroxynitrite in the suppression of mitochondrial respiration in sepsis (132).

Peroxynitrite may further break down cellular energy metabolism by triggering DNA strand breaks, resulting in PARP activation and consumption of cellular NADH, the main reducing equivalent used to support oxidative phosphorylation $(392,624)$. The role of PARP activation in mediating cellular energetic failure has been widely established in septic $(259,276,1046$, $1457,1462)$ and hemorrhagic $(1224,1350,1460)$ models of shock. In humans, evidence for a similar role of PARP has been obtained in septic patients. Investigators measured mitochondrial respiration in cultured human umbilical vein endothelial cells (HUVEC) exposed to serum obtained from healthy patients or patients in septic shock. Compared with healthy serum, septic serum induced a marked reduction of mitochondrial respiration in HUVEC cells, which could be reversed by the NOS inhibitor $N^{\mathrm{G}}$-methyl-L-arginine (L-NMA) or the PARP inhibitor 3-aminobenzamide, implying that a NO/peroxynitrite-dependent activation of PARP is involved in the inhibition of mitochondrial respiration in septic shock (124). In addition to impairing cell energetics, it must also be underscored that PARP may foster the development of inflammation during circulatory shock, by acting as a coactivator of an array of cell signal transduction pathways, and most sigificantly the transcription factor $\mathrm{NF} \kappa \mathrm{B}$, as exposed previously (see sect. V). 


\section{Role of peroxynitrite in the pathophysiological alterations of circulatory shock}

A) PERIPHERAL VASCULAR FAILURE: Circulatory shock is characterized by profound disturbances in cardiovascular homeostasis, affecting all components of the circulation, i.e., the myocardium, the vascular smooth muscle, and the endothelium. In vivo, intense nitrotyrosine immunostaining occurs in the aorta in several models of shock, pointing to significant vascular peroxynitrite formation. Using several pharmacological approaches, peroxynitrite was proven to be an important culprit of peripheral vascular failure in shock, a condition characterized by arterial hypotension and vascular hyporeactivity to vasoconstricting agents. In rat endotoxic shock, both MnTBAP (1457), FeTTPs (265), and melatonin (298a) alleviated vascular hyporeactivity to norepinephrine, while inhibiting aortic peroxynitrite generation (298a,1457). Conversely, enhancing the formation of peroxynitrite by depleting endogenous glutathione with BSO markedly increased vascular hyporeactivity during endotoxic shock (278). Similar observations have been made in hemorrhagic shock: inhibition of peroxynitrite generation using MnTBAP (1231) or MEG (1460) improved aortic contractility and reduced arterial hypotension. Further evidence supports the concept that the final effector mechanism of vascular failure in shock may be due to the peroxynitrite-mediated activation of PARP. Vascular hyporeactivity occurring in experimental models of both septic and hemorrhagic shock was ameliorated by inhibitors of PARP or its genetic deletion (275, $451,623,768,1183,1223,1463)$. Several recent investigations have also shown that peroxynitrite might also affect vascular contractility in shock by altering catecholamine signaling. The systemic administration of peroxynitrite markedly inhibited pressor and vasoconstrictor responses induced by $\alpha$-adrenergic catecholamines in rats (84). In addition, peroxynitrite has been reported to oxidize and inactivate norepinephrine and dopamine, and to block the binding of these catecholamines to their $\alpha$-adrenoreceptors $(1255,1256)$.

B) VASCULAR ENDOTHELIAL DYSFUNCTION: The vascular endothelium plays a number of critical functions in normal vascular physiology, maintaining vascular tone, tuning the balance between procoagulant and anticoagulant factors, and regulating leukocyte adhesion and emigration into the extracellular matrix. Most of these functions are accomplished through the finely regulated release of $\mathrm{NO}$ and prostacyclin $\left(\mathrm{PGI}_{2}\right)$ by endothelial cells $(852,1372)$. These functions are all disrupted during circulatory shock, leading to impaired blood flow regulation and reduced oxygen supply to vital organs.

Villa et al. (1328) were the first to show that infusion of authentic peroxynitrite in isolated perfused hearts resulted in a severe impairment of endothelial-dependent relaxation in coronary vessels. Seminal works by Szabo et al. (1233) then provided evidence that peroxynitritedependent endothelial dysfunction was consecutive to activation of PARP: aortic vascular rings exposed ex vivo to peroxynitrite, as well as vascular rings taken from endotoxemic rats, exhibited reduced endothelium-dependent relaxant responses to acetylcholine that were significantly ameliorated by the PARP inhibitor 3-aminobenzamide. In support of the peroxynitrite/PARP mechanism of endothelial dysfunction, several studies reported an improved endothelial function by pharmacological blockade of peroxynitrite generation $(265,278,1457)$ and PARP activation $(623,1046,1183)$ in rodent septic shock. Peroxynitrite and PARP can trigger multiple cooperating mechanisms to induce endothelial dysfunction in shock. First, peroxynitrite limits endothelial NO production by inactivating eNOS through oxidation of its zinc thiolate center (1469). Second, it oxidizes the NOS cofactor tetrahydrobiopterin $\left(\mathrm{BH}_{4}\right)$, resulting in eNOS uncoupling (725). Third, peroxynitrite potently inactivates $\mathrm{PGI}_{2}$ synthase by tyrosine nitration, resulting in failing prostacyclin synthesis and secondary formation of vasoconstricting prostaglandins such as thromboxane $A_{2}(1469$, 1472). The latter mechanism has been shown to promote vasospasm of coronary vessels treated by endotoxin in vitro (39). Finally, PARP-dependent reduction of cellular NAD may also 
suppress NO formation by depleting endothelial stores of NADPH, an essential cofactor of NOS (430).

C) MYOCARDIAL DEPRESSION: Impaired myocardial contractile function is a welldocumented feature of circulatory shock. It may be triggered by a myriad of mechanisms, including microvascular dysfunction, infiltration by activated leukocytes, and the generation of inflammatory mediators, mainly TNF- $\alpha$ and IL- $1 \beta$, which lead to an overproduction of NO with subsequent cardiodepressive effects. The role of $\mathrm{NO}$ as an inducer of myocardial failure in shock is however controversial, in view of conflicting results indicating either deleterious or beneficial effects of $\mathrm{NO}$ on cardiac function in this setting (729). In fact, sound experimental evidence supports that peroxynitrite might be the link between NO and cardiac depression in shock $(384,604,670,729)$. Overproduction of NO, superoxide, and peroxynitrite occurs in the myocardium during the first $4 \mathrm{~h}$ of endotoxemia, and such changes are correlated with significant reductions of cardiac work, oxygen consumption, and cardiac efficiency (670). In a model of isolated working hearts perfused with TNF- $\alpha$ and IL- $1 \beta$, Ferdinandy et al. (384) reported a steady decline of myocardial contractile function accompanied by enhanced perfusate nitrotyrosine and dityrosine levels. These alterations were markedly attenuated by the peroxynitrite decomposition catalyst FeTPPS. More recently, Lancel et al. (729) showed that rats challenged with endotoxin disclosed myocardial nitrotyrosine formation as well as a severe impairment of cardiac contractile performance, which were almost suppressed by FeTPPS. Although not addressed in these studies, one may speculate that the mechanisms underlying the cardiodepressive effects of peroxynitrite in shock are similar to those reported in other cardiac conditions (heart failure, myocardial infarction, cardiomyopathy), and which include the inhibition of calcium pumps, creatine kinase, mitochondrial enzymes, and $\alpha$ actinin, as well as the activation of MMPs, as detailed in the specific sections on these topics. Additional evidence favoring the role of myocardial PARP activation as an important contributor of peroxynitrite-mediated cardiodepression has been presented. The severe depression of systolic and diastolic left ventricular functions induced by endotoxin in mice is virtually suppressed in mice genetically deficient in PARP (983), and there is a direct correlation between the degree of myocardial PARP activation and the severity of cardiac dysfunction in humans with septic shock (1184).

D) SYSTEMIC INFLAMMATION: Whatever its primary mechanism, circulatory shock rapidly evolves to a state of systemic inflammation, manifested by the de novo generation of an array of proinflammatory and vasoactive mediators, including inflammatory cytokines (e.g., TNF- $\alpha$, IL-6, IL-1 $\beta$ ), chemokines (e.g., IL-8), enzymes (e.g., iNOS, COX2), coagulation proteins, and lipid mediators, to name but a few $(380,544,569,662)$. A key mechanism underlying the upregulation of these mediators lays in the activation of the transcription factor $\mathrm{NF} \kappa \mathrm{B}$, triggered by microbial products (e.g., LPS), inflammatory cytokines, and, supposedly, oxidants and free radicals (759). Peroxynitrite has been proposed to function, in vitro, as an $\mathrm{NF} \kappa \mathrm{B}$ activator, especially in leukocytes, where it was found to stimulate the expression of TNF- $\alpha$ and IL-8 $(391,640,851,1476)$. In vivo, two studies suggested a similar function of peroxynitrite in shock, since the peroxynitrite decomposition catalyst FeTPPS attenuated the activation of $\mathrm{NF} \kappa \mathrm{B}$ in the intestine (803) and the heart (729) of endotoxemic rats. The concept of peroxynitrite-mediated $\mathrm{NF} \kappa \mathrm{B}$ activation has however recently been challenged by results indicating that peroxynitrite inhibited instead of activated $\mathrm{NF} \kappa \mathrm{B}(749,1006,1007)$. Further work must therefore be engaged before any conclusions can be drawn regarding this particular issue. An alternate mechanism whereby peroxynitrite might activate $\mathrm{NF} \kappa \mathrm{B}$ and downstream inflammatory pathways is dependent on PARP. Oliver et al. (973) first demonstrated that the absence of functional PARP suppressed the activation of $\mathrm{NF} \kappa \mathrm{B}$ in immunostimulated cells and that PARP-deficient mice did not exhibit NF $\kappa$ B nuclear translocation when challenged with endotoxin, an effect associated with a markedly reduced release of inflammatory cytokines. These observations have since been confirmed by other investigators $(502,649)$ and recently 
extensively reviewed $(520,1331)$. Overall, the above-presented studies support the contention that peroxynitrite may act to foster inflammatory responses in shock by playing complex roles on the regulation of $\mathrm{NF} \kappa \mathrm{B}$, but additional studies are needed to more precisely assess this point.

E) TISSUE LEUKOCYTE SEQUESTRATION: In addition to its potential roles in the upregulation of $\mathrm{NF} \kappa \mathrm{B}$, peroxynitrite can also participate to the perpetuation of inflammation by enhancing neutrophil activation. In vitro, both exogenous and endogenously synthesized peroxynitrite increased the biosynthesis of IL-8, a major chemoattractant cytokine, by human leukocytes $(641,674,1476)$. Peroxynitrite also enhanced leukocyte endothelial interactions by increasing $\beta_{2}$-integrin at the surface of leukocytes (1477), by stimulating the expression of Pand E-selectins, as well as ICAM-1 in endothelial cells $(272,1118,1178,1447)$, and by impairing endothelial formation of $\mathrm{NO}$ and $\mathrm{PGI}_{2}$ (see above), which are essential for the antiadhesive phenotype of the endothelium. In models of shock elicited by endotoxin or zymosan, the accumulation of neutrophils in tissues such as the heart (729), lung, gut (258, 259), peritoneum (259), and kidney (1418) was dramatically reduced by uric acid (1418), FeTPPS (729), MnTBAP (259), and NAC (258), supporting a similar role of peroxynitrite in vivo. Scavenging peroxynitrite with uric acid also suppressed liver neutrophil accumulation and prevented liver injury in a model of hemorrhagic shock in rats (1296).

F) GUT MUCOSAL BARRIER FAILURE: The loss of the normal barrier function of the gut mucosa is a critical event in shock states. The resulting translocation of microbial products and inflammatory mediators towards the circulation has been proposed as a triggering mechanism of remote organ damage in this setting (944). A contributing role of peroxynitrite to gut barrier failure in shock has been proposed by Salvemini et al. (1109), who showed that various peroxynitrite decomposition catalysts reversed the mucosal injury evoked by the administration of endotoxin to the rat, an effect associated with the suppression of microvascular injury and lipid peroxidation within the intestines. These effects might either reflect direct toxicity of peroxynitrite, or indirect effects related to the activation of PARP. In support of the latter hypothesis, several in vitro studies showed that exposure of human enterocyte monolayers to peroxynitrite was followed by the occurrence of DNA strand breakage and PARP activation. The ensuing functional loss of intestinal barrier function, evaluated by the hyperpermeability to a fluorescent marker, was totally prevented by pharmacological inhibitors of PARP $(202,666,867)$. These conclusions were further supported by the in vivo demonstration that pharmacological inhibition of PARP (1225) or its genetic suppression $(767,768)$ abrogated the intestinal damage and the mucosal hyperpermeability complicating endotoxic (1225) and hemorrhagic shock (767), and suppressed the occurrence of bacterial translocation (1263).

In summary, circulatory shock is a leading cause of death in intensive care units. Considerable improvement in our understanding of the molecular and cellular mechanisms of shock over the past 20 years makes it now a reasonable expectation that novel, efficient mechanism-based therapies will emerge in the near future. Considerable evidence now exists that overproduction of NO and superoxide, triggering the generation of large amounts of peroxynitrite, is a central aspect of shock pathophysiology. In addition to direct cytotoxic effects such as the peroxidation of lipids, proteins, and DNA, peroxynitrite also occupies a critical position in a positive feedback loop of inflammatory injury, by (directly or indirectly, via PARP activation) activating proinflammatory signaling and by triggering the recruitment of phagocytes within injured tissues, leading to further NO, superoxide, and peroxynitrite production, which will progressively amplify the initial inflammatory reactions (see sect. VID, Fig. 14). These various observations support the view that future strategies reducing peroxynitrite or its precursors might have a considerable therapeutic impact in clinical circulatory shock. 


\section{Nitric Oxide and Peroxynitrite in Local Inflammation}

Multiple lines of evidence, gathered in the past 15 years, indicate that enhanced NO production due to induced expression of iNOS by proinflammatory cytokines is instrumental in the pathophysiology of inflammation. Experimentally, the simplest model used to investigate inflammation is the paw edema triggered by the intraplantar injection of the phylogogenic agent carrageenan in rodents, which induces vascular hyperpermeablity and intense infiltration by polymorphonuclear leukocytes (1110). Implication of NO in these changes was initially established by Ialenti et al. in 1992 (592), reporting a marked reduction of edema and vascular hyperpermeability when carrageenan was coinjected with the NOS inhibitors L-NAME or LNMMA. These early observations were then confirmed by the significant prevention of carrageenan-induced paw inflammation in iNOS knockout mice (1352), as well as in rats treated with the selective iNOS inhibitors aminoguanidine and $N$-iminoethyl-L-lysine (L-NIL) (1110). These studies also indicated that carrageenan triggered the formation of nitrotyrosine, which was suppressed by iNOS inhibition, supporting a role for locally produced peroxynitrite in the pathophysiology of inflammation in this model (1110). This hypothesis was elegantly confirmed by Salvemini et al. (1111), who showed that FeTPPS was able to prevent the formation of paw edema and the release of lactate dehydrogenase, a marker of cell necrosis, in rats injected with carrageenan. The key role of iNOS-derived peroxynitrite in this prototypical inflammatory model was then established as a central component of inflammation in more complex paradigms of inflammation, and especially chronic arthritis and inflammatory bowel diseases, as summarized below.

1. Chronic arthritis-Inflammation stands in the foreground of a large number of chronic conditions that have the joints as one of their principal targets. These conditions include rheumatoid arthritis (RA), juvenile chronic arthritis, ankylosing spondylitis, and systemic lupus erythematosus. The most prevalent is RA, which exists in $\sim 1 \%$ of the adult population $(1116,1222)$. The pathogenesis of RA involves the early invasion of the synovial membrane by $\mathrm{T}$ lymphocytes, which activate resident cells (monocytes and synovial fibroblasts) to produce large amounts of proinflammatory cytokines, mainly TNF- $\alpha$ and IL- $1 \beta$, leading to proliferation and neovascularization of the synovial membrane, activation of osteoclasts, and generation of oxidants and free radicals, eventually resulting in the progressive destruction of cartilage (396).

Several lines of evidence indicate that an overproduction of $\mathrm{NO}$ contributes to the pathogenesis of chronic arthritis $(537,876,955)$. In rodent models of arthritis (e.g., induced by Freund adjuvant or type II collagen), increased levels of nitrite/nitrate in plasma $(227,256,398,857)$ and in articular fluid $(1203,1354)$, as well as a high expression of iNOS in hyperplastic synovium $(227,856)$ and chondrocytes $(1419)$, have been demonstrated. In humans, increased circulating levels of nitrate/nitrite are present in arthritic patients (377), and the synovial tissues of patients with RA express iNOS $(860,1103)$ and produce abnormally high amounts of NO (227). The pathogenic role of the increased production of NO in arthritis has been attested by pharmacological studies evaluating the effects of NOS inhibitors. In rat adjuvant arthritis, development of the disease was ameliorated by the nonselective inhibitors L-NAME or LNMMA $(592,979)$, by the iNOS selective inhibitors L-NIL or aminoguanidine (227), and trimethoxy-trifluoromethylchalcone (AEITU) (1419), by PPA250, which suppresses the activity of iNOS by preventing its dimerization (970), and by aminoethyl-isothiourea (ttCH), which downregulates iNOS expression (1082). Similar beneficial effects have been obtained in mouse collagen-induced arthritis with the use of GW274150, a highly potent and long-acting selective inhibitor of iNOS (256) and PPA250 (970). Interestingly, one study reported that the partially selective nNOS inhibitor 7-nitroindazole was beneficial in rat adjuvant arthritis (1039), suggesting a pathological role of this NOS isoform, in addition to iNOS, in the development of joint inflammation in this animal model. Further confirming the 
pharmacological studies, iNOS knockout mice were found to be relatively resistant to collageninduced arthritis, as shown by reduced joint inflammation and paw swelling, and by less structural damage of large joints, both radiographically and histologically (256).

A series of experiments have indicated that peroxynitrite may represent the final executioner of NO-dependent cytotoxicity and inflammation in arthritis. Increased nitrotyrosine formation has been observed in cartilage and subchondral bone of rodents suffering from adjuvant and collagen-induced arthritis $(256,1241,1419)$. The critical role of iNOS-derived NO has been proven by the finding of a complete absence of nitrotyrosine in articular tissues of iNOSknockout mice challenged with collagen (256). Furthermore, the SOD mimetics M40403 also reduced nitrotyrosine formation and joint inflammation in a rat model of collagen-induced arthritis (1108). There is additional evidence of protein nitration in joints from rheumatoid patients, who disclose increased levels of nitrotyrosine in plasma and synovial fluid, and nitrotyrosine formation localized within macrophages and vascular smooth muscle in the inflamed synovium $(842,1115)$.

An early pharmacological study using mercaptoethylguanidine, an anti-inflammmatory compound with combined mechanism of action as a selective iNOS inhibitor and peroxynitrite scavenger, showed an almost complete reversal of collagen-induced arthritis in mice receiving the drug (129). A more thorough investigation of the role of peroxynitrite has been brought up by Mabley et al. (819), who evaluated the effects of FP-15, a novel porphyrin-base peroxynitrite decomposition catalyst, in a mouse model of collagen-induced arthritis. Treatment with FP-15, started at the time of the second collagen injection, markedly reduced both arthritis incidence and severity, as shown by decreased joint swelling and redness of the paws. FP-15 alleviated malondialdehyde accumulation (a marker of lipid peroxidation) and myeloperoxidase activity (indicative of neutrophil infiltration) in affected joints, together with a significant reduction of the local concentrations of the Th1 cytokine IL-12 and of the chemokine MP-1alpha. Importantly, reduced inflammation was also noted, although to a lesser extent, when FP-15 was started 7 days after collagen injection, indicating that NO, superoxide, and peroxynitrite generation represent delayed phenomena in the immunologic and inflammatory series of events associated with arthritis (819).

An important mechanism whereby peroxynitrite may sustain inflammatory changes in arthritis has been proposed to rely on the induction of DNA oxidative damage and subsequent activation of PARP (see sect. IV). In support of this hypothesis, a human study performed in patients with RA reported a significant elevation of DNA strand breaks in peripheral mononuclear cells compared with healthy subjects (99). A strong activation of PARP has been demonstrated in joint tissues from rodents with collagen-induced arthritis, and this activation was suppressed when peroxynitrite formation was blocked by treatment with a selective iNOS inhibitor or by genetic iNOS suppression (256). Further evidence for a pathogenic role of PARP has been given by the considerable reduction of joint inflammation provided by various PARP inhibitors, including nicotinamide (875), 5-iodo-6-amino-1,2-benzopyrone (1241), and PJ-34 (818) in different rodent models of arthritis. Overall, these different sets of data clearly implicate NO, peroxynitrite, and PARP in the pathophysiology of arthritis and indicate that novel, potent peroxynitrite decomposition catalysts and PARP inhibitors might become important therapeutic agents for the treatment of chronic joint inflammation in years to come.

2. Inflammatory bowel diseases-The second major chronic inflammatory process deserving discussion here is represented by the inflammatory bowel diseases (IBDs), which encompass two entities, ulcerative colitis and Crohn's disease (1123). IBD pathogenesis relies on dysregulated interactions of the host mucosal intestinal immunity with the huge antigenic load represented by the microorganisms present in the gut lumen (395), a concept supported by the fact that inflammation does not develop in a germ-free environment in animal models 
of IBD. The importance of proinflammatory cytokines in IBD has been highlighted by the therapeutic successes obtained with anti-TNF antibodies in patients with Crohn's disease (513). A major mechanism underlying the deleterious effects of cytokines in IBD is represented by the induction of iNOS and subsequent overproduction of NO in the injured intestine. In chemically induced models of IBD in rodents, triggered by the intrarectal administration of trinitrobenzene sulfonic acid (TNBS) or acetic acid in ethanol, as well as by the addition of high-molecular-weight dextrans to the drinking water, increased expression and activity of iNOS $(562,1426)$ have been documented in the inflamed colon. Also, in rhesus monkeys spontaneously developing symptoms and histopathological features close to those of human ulcerative colitis, there is a high expression of iNOS and high NO production in the colic mucosa (105). Similarly, in humans, increased expression or activity of iNOS, as well as elevated levels of luminal nitrite have been detected in patients suffering from ulcerative colitis and Crohn's disease $(801,874)$.

A number of studies using NOS inhibitors with differing isoform selectivity have helped to understand the roles of NO in experimental IBD. In rat models of TNBS-induced colitis, nonselective NOS inhibitors (which block the activity of all NOS isoforms) produced contrasting results, with improvements of local inflammation and morphological mucosal damage in some studies, but with opposite results (i.e., increased mucosal inflammation and injury) in others $(322,683,1401)$. Importantly, in the latter studies, treatment was started before or simultaneously with colitis induction. In contrast, NOS inhibition was beneficial when treatment was started after the induction of colitis $(557,683,929)$. In dextran-induced colitis in mice, inflammation was also exacerbated by the prophylactic administration of a massive, nonselective, dose of aminoguanidine (956), while administration of the highly selective iNOS inhibitor 1400W (714) was protective. It is also important to mention that iNOS knockout mice were largely protected against colitis induced by dextran $(562,714)$ and TNBS/ethanol (1464). In the latter study, iNOS knockout mice showed a smaller loss of body weight, an attenuated morphological damage to intestinal epithelium, less mucosal immunostaining for nitrotyrosine, and a lower colic content in malondialdehyde, indicative of a reduced level of nitrative and oxidative stress in the colon of knockout animals. Overall, these various results suggest an important protective role of $\mathrm{NO}$ generated by the constitutive isoforms of $\mathrm{NO}$ at the early stage of colitis, possibly through the preservation of mucosal perfusion and/or mitigation of leukocyte recruitment. Alternatively, the strong induction of iNOS that occurs at a more advanced stage contributes to inflammation and mucosal damage in this model.

Supporting a role for iNOS-dependent generation of peroxynitrite in the pathogenesis of IBD, biochemical evidence of peroxynitrite generation (formation of nitrotyrosine) has been shown both in experimental models of colitis $(885,1170,1464)$ and in human samples of active Crohn's lesions, in which massive nitrotyrosine immunostaining has been reported (1170). The formation of peroxynitrite in such circumstances is largely favored, since not only NO but also superoxide are being formed in the inflamed intestines $(268,321)$, which explains the beneficial effects of SOD mimetics in experimental colitis (268). In addition, it is noteworthy that the direct application of authentic peroxynitrite within the gut elicits a severe, colitis-like, inflammatory response (1052). The pathogenic role of peroxynitrite has been firmly established by studies using pharmacological approaches to reduce peroxynitrite in the inflamed colon. Thus treatment of rats with the iNOS inhibitor and peroxynitrite scavenger mercaptoethylgaunidine markedly alleviated colonic injury induced by intrarectal instillation of TNBS (1456). Also, the peroxynitrite decomposition catalyst FP-15 exerted potent protective effect in mice fed with 5\% dextran sulfate sodium (819). Treatment with this compound, when started simultaneously with dextran, largely reduced colon damage and inflammation, as assessed by histological evaluation, and reduced rectal bleeding, weight loss, and colon shortening. Furthermore, FP-15 reduced the colitis-induced increase in colonic malondialdehyde levels, indicative of the ability of the drug to attenuate oxidative stress, and 
reduced colonic infiltration by activated phagocytes, as determined by reduced myeloperoxidase levels (819).

As in the case of arthritis, there is large evidence that a common final pathway of peroxynitritedependent inflammatory changes in IBD depends on the activation of PARP. First, PARP is strongly activated in various rodent models of colitis, and second, pharmacological inhibition of PARP or PARP genetic suppression provided significant resistance against the development of colitis in these models (see Ref. 1230 for recent review). In addition to preventing cell necrosis by inhibiting ATP depletion, it has also been established that the absence of PARP exerted direct anti-inflammatory effects in TNBS-induced colitis as well as in the spontaneous colitis that develop in IL-10-deficient mice, as shown by decreased neutrophil infiltration, reduced release of proinflammatory cytokines and chemokines, and smaller increases in colon permeability $(634,818,1461)$. In a recent work in mice challenged with intrarectal TNBS, reduced colon ulceration and apoptotic mucosal cell death were noted in PARP knockout mice and could be correlated with a reduced activation of the MAPK JNK. This was followed by a depressed activity of the transcription factor AP-1 in the colon, together with an enhanced expression of the antiapoptotic protein Bcl-2 (1458). These results represent a further illustration of the very complex interactions occurring in vivo between PARP, cell death, and inflammation, as exposed in detail in section IV.

3. Tissue inflammation from toxic origin-In addition to its involvement in the pathophysiology of well-characterized acute and chronic inflammatory diseases, peroxynitrite is also suspected to play an important role in the development of organ damage and inflammation triggered by various drugs and chemical agents. A detailed description of these emerging roles of peroxynitrite in drug-dependent toxicity is beyond the scope of this review, and further informations can be obtained in an extensive review on this topics recently published by Denicola and Radi (311). Two drugs deserve here some discussion, in view of their widespread use in clinical medicine, namely, doxorubicin (DOX) and acetaminophen.

DOX is an anthracycline antibiotic used as an antitumor agent, whose major side effect is significant cardiotoxicity (985). DOX forms a complex with cardiolipin within the mitochondrial inner membrane of cardiomyocytes, where it is reduced by NADH dehydrogenase from the respiratory chain to form a semiquinone radical, which is then oxidized back to the parental compound by passing the electron received onto molecular oxygen, forming a superoxide radical (843). The superoxide formed during the redox cycling of DOX rapidly combines with $\mathrm{NO}$ to generate peroxynitrite, as indicated by the intense nitrotyrosine staining demonstrated in rodents treated with DOX $(985,1355)$ (Fig. 13C). Importantly, a direct correlation between the degree of myofibrillar protein nitration and the severity of DOXinduced heart failure has been established in these models (1355). The critical role of peroxynitrite downstream in DOX-mediated myocardial dysfunction has been firmly established by Pacher et al. (985), who found that accelerating peroxynitrite decomposition with FP-15 produced a significant protection against the protein nitration, lipid peroxidation, MMP activation, and cardiac dysfunction triggered by DOX treatment in mice.

Acetaminophen (AAP), a widely used analgesic drug, can cause severe liver injury in the overdose setting (622). AAP toxicitiy is mainly related to its metabolic activation to the reactive metabolite $N$-acetyl-p-benzoquinone imine (NAPQI), which consumes hepatic glutathione and subsequently covalently binds to cellular proteins, notably within mitochondria (622). These early events are rapidly followed by disturbances of $\mathrm{Ca}^{2+}$ homeostasis (235), inhibition of mitochondrial respiration (620), overproduction of $\mathrm{O}_{2}{ }^{--}$and $\mathrm{NO}$ (due to iNOS expression; Ref. 432) and subsequent generation of peroxynitrite (622). A strong immunoreactivity for nitrotyrosine, mainly in the mitochondria, has been detected in dying hepatocytes after acetaminophen overdose $(239,690)$. Furthermore, administration of GSH (46) or NAC 
(1071) to restore endogenous GSH stores has been shown to reduce nitrotyrosine staining and to attenuate liver injury upon AAP overdose in animals. According to our current understanding, mitochondrial peroxynitrite formation in response to AAP triggers massive hepatocyte cell death by inducing membrane permeability transition, collapse of the mitochondrial membrane potential, and depression of ATP biosynthesis (622). Additional studies with peroxynitrite decomposition catalysts are needed to further precisely define these possible roles of peroxynitrite in AAP overdose.

In summary, studies performed in animal models of inflammatory diseases have highlighted the crucial role of the NO-superoxide-peroxynitrite-PARP activation pathway as a mechanism of progressive amplification of inflammation in these conditions. Such a role is certainly not limited to arthritis and colitis, as similar roles of peroxynitrite and PARP have been documented in many other localized inflammatory paradigms, recently reviewed by Szabo (1230). These include inflammation of the CNS, such as allergic encephalomyelitis, meningitis, and multiple sclerosis; ocular inflammation (uveitis); asthma; and periodontal inflammation (gingivitis), as well as the systemic inflammatory responses observed during circulatory shock (see sect.

VIC for specific discussion). As summarized in Figure 14, it is currently envisioned that the generation of peroxynitrite initiates oxidative DNA damage and DNA strand breaks formation in all these conditions. In turn, injured DNA triggers PARP activation in cells exposed to toxic concentrations of peroxynitrite. Activation of PARP depletes cellular NAD ${ }^{+}$and ATP, leading, in the worst case, to irreversible damage and necrotic cell death. Simultaneously, PARP acts as a coactivator of multiple crucial proinflammatory cascades, including MAPKs, AP-1, and $\mathrm{NF} \kappa \mathrm{B}(361,1230)$. This results in a greater expression of proinflammatory cytokines, chemokines, enzymes (e.g., iNOS), and adhesion molecules. Inflammatory cells are sequestered in the tissues, enhancing the production of oxidants and free radicals, generating further DNA damage and PARP activation, thereby fueling a continuous cycle of progressive inflammation. The different events set in motion by the initial generation of peroxynitrite indicate that potent peroxynitrite decomposition catalysts and PARP inhibitors might represent useful therapeutic agents for debilitating chronic inflammatory diseases.

\section{E. Nitric Oxide and Peroxynitrite in Cancer}

A relationship between chronic inflammation and tumorigenesis has long been suspected. It is well known that malignant tissues are infiltrated by leukocytes, which locally secrete cytokines, chemokines, matrix-degrading enzymes, growth factors, free radicals, and oxidants. This creates a microenvironment that may enhance cell proliferation, survival, and migration, as well as angiogenesis, thereby promoting tumor development (238). A particularly important role of increased $\mathrm{NO}$ generation in this microenvironment is now well recognized as an essential step initiating neoplastic transformation (418). Importantly, not only immune cells infiltrating the tumor, but also tumor-associated stromal fibroblasts and the tumor cells themselves, notably in lung, breast, thyroid, stomach, and colon cancers, are able to produce large amounts of NO due to induced expression of iNOS (see Ref. 418 for review). The latter can be triggered in cancer cells via cytokine-dependent $\mathrm{NF} \kappa \mathrm{B}$ signaling, or via a signaling cascade involving activation of the transcription factor HIF-1 under the hypoxic conditions which may prevail in rapidly growing tumors. In addition to iNOS, several types of tumor cells, for example, from breast, brain, or lung origin, also express eNOS or nNOS, while endothelial cells in the tumor vasculature produce eNOS-derived NO (418).

Evidence for a role of $\mathrm{NO}$ overproduction as a mechanism initiating and promoting tumorigenesis has come from studies showing reduced tumor formation by genetic deletion of iNOS in several mouse models of cancer. For instance, iNOS knockout mice had an $80 \%$ reduction of urethane-induced lung tumor formation (682). Similarly, mice with a mutation of the adenomatous polyposis coli (Apc) gene, who spontaneously develop multiple polyps in the 
intestines, are protected from polyp development when bearing an iNOS knockout genotype, or when treated with a selective iNOS inhibitor (9). Several mechanisms accounting for the tumor-promoting influence of NO have been identified. First, NO can stimulate tumor angiogenesis, by inducing angiogenic and lymphangiogenic factor expression, most significantly vascular endothelial growth factor (VEGF) $(18,19,631)$, by inhibiting the expression of endogenous antiangiogenic factors such as thrombospondin-1 (1076), and by stimulating blood vessel maturation via the recruitment of perivascular cells (pericytes) (657, 1423). Second, NO has been associated with enhanced migration and invasion of tumor cells through mechanisms depending on guanylyl cyclase and MAPK signaling, notably in breast and colon cancer cells $(619,975,1163)$. Third, NO can induce DNA damage, potentially leading to DNA mutations and clonal transformation (418). As discussed below, evidence is emerging that peroxynitrite most probably represents the major species responsible for such DNA mutations linking NO overproduction with carcinogenesis.

Studies pointing to a tumor-promoting role of NO must be weighted by the contrasted results of studies that showed instead a tumor-suppressing activity of NO. Indeed, several reports indicated prevention of intestinal tumorigenesis, as well as sarcomagenesis and lymphomagenesis in iNOS knockout mice $(314,585,1140)$. It is currently considered that these contrasted influences of NO depend on the duration and level of NO exposure, the type of iNOS-expressing cell (tumor vs. stromal or inflammatory cells), the cellular sensitivity to NO's cytotoxic activity, and the status of the p53 tumor suppressor gene in the tumor cell (418). Obviously, more studies will be necessary to precisely delineate the role of NO in specific forms of tumors, especially when considering the potential clinical application of strategies aimed at either inhibiting, or enhancing, NO generation, as an adjuvant therapy in human cancer. A detailed discussion on the multifaceted actions of NO in cancer, as well as possible future therapeutic implications, is beyond the scope of this article but can be found in an extensive review recently published by Fukumara et al. (418). In the next paragraphs, we limit our focus on the evidence favoring a potential role of peroxynitrite in carcinogenesis.

As discussed in section IV, there are abundant demonstrations of the toxic consequences of peroxynitrite towards DNA. Peroxynitrite triggers oxidative modifications within nucleobases, most significantly guanine (945), generating 8-oxoguanine, which is at least 1,000-fold more reactive with peroxynitrite than parent guanine, yielding further oxidation products such as spiroiminodihydantoin, guanidinohydantoin, cyanuric acid, and oxazolone $(945,1124)$. In addition to oxidative damage, peroxynitrite can also nitrate guanine, to form 8-nitroguanine and 5-guanidino-4-nitroimidazole $(945,1124)$. Overall, the guanine modifications introduced by peroxynitrite have been shown to result in a series of potential mutations in both viral (939) and mammalian (1219) DNA (G to A, G to T, and G to C mutations). Peroxynitrite further damages DNA by producing DNA single-strand breaks, due either to endonuclease-dependent cleavage of abasic sites created by peroxynitrite, or to direct oxidation of the deoxyribose moiety, which opens the sugar ring $(160,945,1422)$. Peroxynitrite-mediated guanine oxidation and single-strand breaks have been identified as powerful mechanisms of homologous recombination in DNA (684), which is recognized as a critical step in the induction of genomic lesions associated with carcinogenesis. In addition to directly targeting DNA, peroxynitrite may promote DNA damage by inactivating DNA repair enzymes, e.g., OGG1 (8-oxoguanine DNA glycosylase), a key base excision repair enzyme responsible for base excision repair of 8-oxoguanine (625), as well as by inhibiting (through tyrosine nitration) the transcription factor p53 (222,223), which serves as an essential mechanism to remove cells having sustained significant DNA damage.

The considerable genotoxic potential of peroxynitrite detected in cell culture studies and purified DNA supports the contention that intense and prolonged peroxynitrite-dependent oxidative and nitrative stress prevailing under inflammatory conditions might foster the 
development of cancer. This hypothesis has been strengthened by the in vivo demonstration of 8-nitroguanine formation in chronic infections and inflammatory diseases known to be associated with greater risk of cancer. These include chronic infection of the bile ducts with the liver fluke in hamster (a risk factor for cholangiocarcinoma) (1029), chronic gastric infection with $H$. pylori in humans (which may precede gastric carcinoma) (813), human oral leukoplakia (a precancerous state) (814), and chronic colitis in mice (associated with the development of colon carcinoma) (324). In the latter study, the important finding was made that 8-nitroguanine formation in colon epithelial cells colocalized with the expression of proliferating cell nuclear antigen, a marker of cell proliferation, indicating that nitrative DNA damage induced in colon epithelial cells during chronic colitis is associated with proliferation of these cells, which may contribute to colon carcinogenesis (324). More direct evidence of the tumor-promoting role of peroxynitrite was recently described in mice with $A p c$ gene mutation ( $A p c^{\mathrm{min}}$ mice). A considerable reduction in the spontaneous development of intestinal polyps (evaluated at $12 \mathrm{wk}$ of age) occurred when $A p c^{\min }$ mice were treated by the peroxynitrite decomposition catalyst FP15, given orally from the fifth week of life. FP-15 also resulted in significant reduction of malondialdehyde accumulation in intestinal tissues, pointing to a key role of peroxynitrite-related oxidative stress in tumor initiation in this particular model (820).

In summary, available evidence indicates that NO plays dichotomous roles (promotion vs. suppression) in tumor initiation and progression. The activation of angiogenesis and the induction of DNA mutations represent key aspects of the procarcinogenic effects of NO. Peroxynitrite is emerging as a major NO-derived species responsible for DNA damage, mainly through guanine modifications and the inhibition of DNA repair enzymes. In chronic inflammatory states, the identification of 8-nitroguanine in tissues indicates that nitrative DNA damage consecutive to overproduction of $\mathrm{NO}$ and peroxynitrite may represent an essential link between inflammation and carcinogenesis.

\section{F. Nitric Oxide and Peroxynitrite in Stroke and Other Forms of Reperfusion Injury}

1. Stroke-Acute ischemic stroke is the second leading cause of death in industrialized countries and the leading medical cause of acquired adult disability. In the United States, $\sim 600,000$ new strokes occur annually, representing an economic burden of $\$ 40$ billion in direct and indirect costs. Ischemic stroke results from the reduction of cerebral blood flow in the territory of a major cerebral artery due to its transient or permanent occlusion by local thrombosis or embolus. A complex cascade of molecular events is set in motion during cerebral ischemia and culminates in neuronal cell death. Improving our understanding of these events might help devise novel therapies to limit neuronal injury in stroke patients, a concept termed "neuroprotection" (741,1363).

In both animal models of focal cerebral ischemia [e.g., induced by middle cerebral artery (MCA) occlusion] and in human stroke, two distinct ischemic regions can be identified: the core, which is served primarily by the occluded artery, and the penumbra, where residual blood flow ( $<40 \%$ of normal flow) persists $(326,1363)$. The earliest consequence of ischemia is a significant fall in tissue ATP levels, which is considerably more pronounced in the core than in the penumbra. The immediate consequence is the dissipation of ionic gradients normally maintained by $\mathrm{Na}^{+}-\mathrm{K}^{+}$-ATPases, resulting in the rapid depolarization of neurons and glia, followed by the activation of voltage-dependent $\mathrm{Ca}^{2+}$ channels and $\mathrm{Ca}^{2+}$ overload (778). These events induce the accumulation of excitatory neurotransmitters, especially glutamate, within the extracellular space, triggering the activation of two distinct ionotropic receptors, the NMDA and $\alpha$-amino-3-hydroxy-5-methyl-4-isoxazole (AMPA) receptors. NMDA receptor opens a $\mathrm{Ca}^{2+}$ channel and thus amplifies $\mathrm{Ca}^{2+}$ overload, whereas AMPA receptor opens a $\mathrm{Na}^{+}$channel, leading to accumulation of intracellular $\mathrm{Na}^{+}$and brain edema $(326,778)$. The pathological 
alterations elicited by the actions of glutamate on these receptors are referred to as "excitotoxicity."

The massive increase in intracellular $\mathrm{Ca}^{2+}$ activates various enzymes (e.g., phospholipases, cyclooxygenases, NO synthase, and proteolytic enzymes) and stimulates the release of cytotoxic oxidant species and free radicals, which provoke lipid peroxidation, DNA injury, and mitochondrial damage, and also contribute to the breakdown of the blood-brain barrier and the development of brain edema $(326,778,1079)$. Furthermore, tissue anoxia, calcium overload, and oxidant stress foster the activation of various transcription factors, including $\mathrm{NF} \kappa \mathrm{B}$, hypoxia-inducible factor 1, and STAT3. This, in turn, triggers the production of inflammatory cytokines (TNF- $\alpha$, IL-1 $\beta$ ), enzymes (iNOS, COX-2), adhesion molecules (ICAM-1, selectins), and the recruitment of activated phagocytes $(326,587)$. These changes evolve over several days after the initial ischemic insult, resulting in progressive inflammatory changes spreading from the core into the ischemic penumbra and characterized by the migration of activated microglial cells, macrophages, and neutrophils within the site of injury, where they produce large quantities of oxidants and free radicals. Ultimately, $\mathrm{Ca}^{2+}$ overload, glutamate excitotoxicity, oxidant-mediated injury, and inflammatory changes induce neuronal cell death, which occurs predominantly by necrosis within the core of the lesion, and by apoptosis within the penumbra (326).

\section{A) ROLE OF NO IN NORMAL BRAIN PHYSIOLOGY AND DURING ISCHEMIC} STROKE: In the brain, $\mathrm{NO}$ is physiologically produced by two $\mathrm{Ca}^{2+} /$ calmodulin-dependent NOS isoforms present in endothelial cells (eNOS, type III NOS) and neurons (nNOS, type I NOS), which produce small (nanomolar) amounts of NO for short periods in response to transient increases in intracellular $\mathrm{Ca}^{2+}$. NO generated by eNOS and nNOS is essential for the control of cerebral blood flow, whereas nNOS-derived NO also functions as a neurotransmitter and is involved in synaptic plasticity, modulation of neuroendocrine functions, memory formation, and behavioral activity $(491,1229)$. Under various pathological conditions, including cerebral ischemia, large amounts of $\mathrm{NO}$ are produced in the brain as a result of the induced expression of iNOS, related to enhanced iNOS gene transcription in response to locally produced inflammatory cytokines. Expression of iNOS in brain tissue has been localized in infiltrating phagocytes, vascular cells, and glial cells (astrocytes, oligodendrocytes, and microglia) $(39,491)$.

A number of experimental investigations have shown that NO production is upregulated and plays opposite (protective vs. deleterious) effects in stroke. In both focal and global cerebral ischemia models in rodents, a burst of $\mathrm{NO}$ generation, occurring in the first minutes of ischemia, was demonstrated by EPR spectroscopy $(834,1282)$ and the determination of NO-

decomposition products (nitrites and nitrates) (1156; see Refs. 110 and 906 for review). In the rat forebrain, $\mathrm{NO}$ levels up to $11 \mu \mathrm{M}$ have been determined following global ischemia induced by bilateral carotid occlusion (1282). Ischemia-induced $\mathrm{NO}$ overproduction has been correlated with glutamatergic-mediated increases in intracellular $\mathrm{Ca}^{2+}$ concentrations, resulting in a calmodulin-dependent upregulation of nNOS and eNOS activities in the infarcted tissues $(110,906)$. This increased activity is short lasting $(\sim 1 \mathrm{~h})$, declining rapidly to $<25 \%$ of control activity after a few hours in both transient and permanent ischemia $(466,588)$. Importantly, this reduced activity persists for at least 10 days after the induction of focal ischemia (466), a phenomenon supposed to reflect the loss of nNOS-expressing neurons, the degradation of nNOS within the infarct (101), and the inhibition of nNOS activity by iNOS-derived NO in the late phase of ischemia (907). In addition to stimulated NOS activity, there is a transient increase in the expression of both nNOS (1443) and eNOS (1444) in the ischemic area, which might contribute to the initial burst of NO in stroke (907). 
Following this early and transient rise in NO formation, a secondary wave of NO overproduction starts to develop several hours after the initial ischemic insult and is sustained for up to $4-7$ days $(466,588)$. This enhanced and prolonged NO release can be entirely ascribed to the induced expression of iNOS, which has been detected in infiltrating neutrophils, cerebral blood vessels (589), and glial cells, especially astrocytes (101), in rodent models of transient or permanent ischemia (reviewed in Refs. 111,906). In humans, it is likely that a similar scenario takes place, in view of the increase in the levels of nitrates and nitrites in blood and cerebrospinal fluid of stroke patients $(181,346,716)$, together with the demonstrated expression of iNOS in neutrophils and blood vessels in autopsy brain specimens (404).

Using both pharmacological (administration of NOS inhibitors with various isoform specificity) and genetic approaches (using mice with targeted deletions of the eNOS, nNOS, or iNOS gene), the role of each NOS isoform in cerebral ischemia has been investigated. It has thus been clearly demonstrated that NO plays both neuroprotective and neurotoxic roles in stroke, depending on the NOS isoform from which it is produced (491). Thus eNOS-derived NO produces beneficial effects, as demonstrated by the larger infarcts measured $24 \mathrm{~h}$ after MCA occlusion in mice lacking the eNOS gene (575). Vascular NO production dependent on eNOS has been proposed to protect against stroke mainly by increasing collateral blood flow to the ischemic penumbra $(1106,1432)$, but additional mechanisms have also been suggested. These include the enhancement of endothelium-dependent vasorelaxation, the prevention of inflammation and oxidative damage, the inhibition of platelet aggregation and thrombosis formation, the prevention of neuronal apoptosis, the reduction of NMDA receptor activation, the mobilization of stem and progenitor cells, and the improvement of neovascularization, as recently reviewed by Endres et al. (358). In line with the beneficial effects attributed to eNOSderived NO, numerous studies reported that administration of the eNOS substrate L-arginine increased regional cerebral blood flow in the ischemic territory, improved functional recovery, and conferred protection against stroke (reviewed in Ref. 1374). Similarly, the enhancement of eNOS expression by the administration of statins $(357,1173)$ or corticosteroids $(505)$, as well as treatment with various NO donors $(672,1374)$, have been associated with neuroprotective effects in rodent models of transient or permanent MCA occlusion.

In marked contrast to the beneficial effects afforded by eNOS, both nNOS and iNOS have been associated with deleterious, neurotoxic effects in stroke. Mice lacking either the nNOS (390, 576) or iNOS (590) gene are protected against ischemic damage after both transient or permanent ischemia, and similar beneficial effects have been obtained with pharmacological NOS inhibitors exhibiting selectivity towards nNOS, e.g., 7-nitroindazole (1421), ARL17477 (1445), and BN80933 (193) or iNOS, such as aminoguanidine (591) or $1400 \mathrm{~W}$ (1008).

Importantly, the therapeutic window for administration of selective iNOS inhibitors in these studies was much longer than that for nNOS inhibitors, given the time course of iNOS expression after brain ischemia. iNOS inhibitors still provided protection when given as late as $24 \mathrm{~h}$ after MCA occlusion (907). Of note, treatment with nonselective NOS inhibitors (e.g., L-NAME or $N^{\mathrm{G}}$-nitro-L-arginine) yielded much more variable outcomes in experimental stroke, due to their inherent inhibitory action on eNOS. Studies with these agents reported either beneficial, neutral, or detrimental effects, depending on the experimental model (transient vs. permanent ischemia) and the timing of administration (before or after the induction of ischemia), as reviewed recently (1373).

In summary, the different studies listed above indicate that small amounts of NO produced by eNOS in the vasculature during the early phase of brain ischemia are essential to limit the extent of cerebral damage, whereas higher concentrations of NO, generated initially by nNOS and later by iNOS, exert essentially neurotoxic effects in the ischemic brain. Evidence that such toxicity depends, in large part, on the rapid reaction of $\mathrm{NO}$ with locally produced superoxide to generate peroxynitrite will be now exposed (see also Fig. 15.) 


\section{B) ROLE OF PEROXYNITRITE IN NO-DEPENDENT NEUROTOXICITY IN}

STROKE: Seminal studies on the possible neurotoxic role of NO were conducted in the late 1980s by Bredt and Snyder (134), who showed that exposure of cerebellar slices to the excitotoxic neurotransmitters glutamate and NMDA triggered the formation of NO. Two years later, Dawson et al. (295) definitely identified NO as the mediator of glutamate and NMDAdependent neurotoxicity, by showing that NOS inhibitors prevented NMDA-dependent neuronal cell death in vitro. The toxic role of NO did not appear straightforward, however, as NO was simultaneously found to exhibit protective effects, by inhibiting responses mediated by NMDA receptors in rat cortical neurons (746). This paradox was resolved in 1993 by Lipton et al. (779), who showed that NO-mediated neurotoxicity was engendered by its reaction with superoxide to form peroxynitrite, whereas its protective effects resulted from the downregulation of NMDA receptor activity by $S$-nitrosylation of thiol group(s) in the receptor's redox modulatory site.

In vivo, the generation of peroxynitrite in ischemic stroke is largely favored, due to the simultaneous increase of both NO (see above) and superoxide production. The upregulated formation of superoxide in ischemic stroke has been attested by methods such as cytochrome $c$ oxidation (374), lucigenin enhanced chemiluminescence (1025), and hydroethidine oxidation (677), which showed that levels of superoxide start to rise during the ischemic phase, followed by a much larger increase during the early reperfusion period, both in neurons and in endothelial cells. In focal ischemia, this increase was much sharper in the ischemic penumbra than in the core, reaching up to $25 \mu \mathrm{M} / \mathrm{min}$ (374). The pathogenic importance of superoxide in ischemic stroke has been highlighted by studies using transgenic mice with either deletion or overexpression of the various SOD isozymes. Thus mice deficient in Cu/Zn-SOD (701) or Mn$\operatorname{SOD}(677,915)$ disclosed larger infarcts after transient focal ischemia, whereas mice overexpressing $\mathrm{Cu} / \mathrm{Zn}$-SOD were protected (1410). By the same token, pharmacological compounds exhibiting properties as SOD mimetics were reported to exert significant protection against brain ischemic damage in rodents $(1153,1158,1266)$.

Many different sources act in concert to produce such high concentrations of superoxide in stroke. The most important is probably the mitochondrion (1169), which produces superoxide via the partial reduction of molecular oxygen by electrons leaking from the electron transport chain under conditions of respiratory block, a phenomenon amplified by cellular hypoxia and NO-mediated inhibition of cytochrome oxidase $(143,533)$. The central role of mitochondria in generating toxic levels of superoxide during stroke is attested by the demonstration that mice lacking the mitochondrial isoform of SOD (Mn-SOD) experience much larger infarcts than their wild-type littermates after transient MCA occlusion $(677,915)$. Superoxide also arises from the activity of cyclooxygenase enzymes (COX), especially COX-2, present in glutamatergic neurons and which catalyzes the conversion of arachidonic acid to prostaglandin $\mathrm{G}_{2}\left(\mathrm{PGG}_{2}\right)$ and then to $\mathrm{PGH}_{2}$, with the concomitant liberation of superoxide (661). In brain pathologies associated with glutamate excitotoxicity, and notably stroke, COX-2 is markedly upregulated and becomes a significant generator of superoxide, especially in the ischemic penumbra (1448). NADPH oxidase, expressed by neurons $(1147,1310)$, endothelial cells (8), and infiltrating neutrophils (778), represents a further source of superoxide during ischemia, and its blockade, either by pharmacological inhibition or genetic suppression (1337), produces significant reductions of brain infarct volume in experimental stroke. Finally, superoxide may also be generated by the hypoxic-dependent conversion of xanthine dehydrogenase into xanthine oxidase, during the metabolism of the breakdown products (xanthine and hypoxanthine) of adenine nucleotides (778), as well as by the uncoupling of eNOS, due to deprivation of its cofactor tetrahydrobiopterin or its substrate L-arginine, caused by the reduced blood supply (358). In such conditions, eNOS may function as a superoxide generator instead of an $\mathrm{NO}$ generator. 
Evidence for the generation of peroxynitrite from $\mathrm{NO}$ and superoxide in stroke has been obtained by the concomitant demonstration that 1 ) nitrotyrosine rapidly accumulates in the brain following transient or permanent ischemia and 2) the nitrotyrosine accumulation is markedly prevented by strategies blocking NO or superoxide generation, as well as by treatments aimed at directly scavenging peroxynitrite. Although nitrotyrosine can be formed independently from the generation of peroxynitrite, via the reaction of hydrogen peroxide with myeloperoxidase (see sect. IV), this mechanism is highly unlikely to occur in stroke, as shown in a recent study using myeloperoxidase-deficient mice exposed to transient focal cerebral ischemia. Absence of myeloperoxidase did not reduce the accumulation of nitrotyrosine in the infarcted tissue, nor did it reduce the lesion size, indicating that the peroxynitrite pathway, but not the myeloperoxidase pathway, is responsible for the nitration reaction occurring in the ischemic brain (1258).

nNOS plays the predominant role in the formation of nitrotyrosine in the early phase of ischemia and reperfusion, as demonstrated by the suppression of nitrotyrosine generation by 7-nitroindazole, a selective nNOS inhibitor administered in the acute phase (15 min before occlusion) of focal brain ischemia reperfusion in mice (550). Also, the accumulation of nitrotyrosine found in cortical neurons and vascular endothelium after $3 \mathrm{~h}$ of permanent ischemia was absent in nNOS knockout mice, supporting an essential role of nNOS in this early nitrotyrosine accumulation (348). Comparable results were obtained in transient ischemia in rats, where nitrotyrosine formed in cortical neurons could be eliminated by tamoxifen, a compound with nNOS inhibitory activity (976). NMDA receptor activation appears as the major trigger of this early, nNOS-dependent nitrotyrosine formation, in view of the suppression of the latter afforded by MK-801, a NMDA receptor antagonist, in focal transient ischemia (551). Interestingly, although eNOS has generally been attributed a neuroprotective function in stroke, it might also be responsible for the generation of peroxynitrite by triggering a surge of NO at the onset of reperfusion, as recently proposed by Gursoy-Ozdemir et al. (495). These investigators demonstrated that the accumulation of nitrotyrosine in blood vessels after reperfusion was not influenced by the nNOS inhibitor 7-nitroindazole, whereas it was suppressed by the eNOS inhibitor nitro-L-arginine $(494,495)$. These data indicate that, although NMDA-dependent nNOS activation represents the major source of peroxynitrite in neurons, eNOS may play this role in the vascular endothelium during the early reperfusion phase.

At later stages of stroke, formation of peroxynitrite is totally dependent on the expression of iNOS. In a model of transient MCA occlusion in mice, strong nitrotyrosine immunoreactivity was demonstrated within the vascular wall in the peri-infarct region, peaking $15 \mathrm{~h}$ after reperfusion. Nitrotyrosine staining was totally absent in iNOS knockout mice (552). In rats exposed to $2 \mathrm{~h}$ of MCA occlusion followed by $22-70 \mathrm{~h}$ of reperfusion, nitrotyrosine peaked at $48 \mathrm{~h}$ within vascular walls and then progressively declined. The time course of nitrotyrosine paralleled the infiltration of neutrophils within the ischemic region, suggesting a major role of iNOS and superoxide derived from infiltrating phagocytes in the formation of peroxynitrite in this model (1218). Similarly, Bidmon et al. (101) found that nitrotyrosine formation in blood vessels from the border of focal cortical ischemic lesions in rats occurred simultaneously to the induced expression of iNOS. The latter was detected after 1 day in neutrophils, accumulating in injured blood vessels and infiltrating the perilesionnal cortex, and after 3 days in microglia, astrocytes, and layers V and VI pyramidal neurons (101). The formation of nitrotyrosine can be considerable, as indicated in a study by Takizawa et al. (1260). Using HPLC techniques, these authors found that the ratio of nitrotyrosine to tyrosine reached $0.95 \%$ in the penumbra and $0.5 \%$ in the core of infarct at $1 \mathrm{~h}$ after the onset of reperfusion after MCA occlusion in rats. These elevated levels persisted for $48 \mathrm{~h}$ and were suppressed by the iNOS inhibitor aminoguanidine (1259). The induced expression of iNOS, leading to peroxynitrite formation in the microvasculature, glia, and neurons after transient brain ischemia appears to 
depend in large part on the local generation of the inflammatory cytokine IL-1, as evidenced by the marked attenuation of iNOS and peroxynitrite observed in mice lacking the IL-1 gene (968). Increased nitrotyrosine staining of brain tissue in asphyxiated neonates was also recently demonstrated, suggesting that peroxynitrite toxicity might play a role in hypoxic-ischemic brain injury at term (481).

Once formed in the ischemic brain, peroxynitrite may exert its toxic effects through multiple mechanisms, including lipid peroxidation, mitochondrial damage, protein nitration and oxidation, depletion of antioxidant reserves (especially glutathione), and DNA damage followed by the activation of the nuclear enzyme PARP, as summarized in Figure 15. A series of in vivo experimental studies have specifically addressed the neurotoxicity elicited by peroxynitrite in stroke, by using various pharmacological strategies to remove peroxynitrite in rodent models of brain ischemia.

Dhar et al. (315) investigated the neuroprotective effects of peroxynitrite decomposition catalyst FeTMPyP on global cerebral I/R injury produced by $5 \mathrm{~min}$ of occlusion of both common carotid arteries followed by reperfusion of $96 \mathrm{~h}$ in the adult male Mongolian gerbils. FeTMPyP administered $30 \mathrm{~min}$ prior to ischemia improved the neurological functions, reduced the hyperlocomotion and memory impairment, attenuated neuronal loss from the pyramidal layer of the CA1 region, and inhibited lipid peroxidation in I/R-challenged gerbils (315). Thiyagarajan et al. (1273) found that when peroxynitrite decomposition catalysts FeTMPyP and FeTPPS were given at the time of reperfusion in rats exposed to 2-h MCA occlusion followed by 70 -h reperfusion, both compounds reduced cerebral infarction by $\sim 70 \%$.

Importantly, the drugs were still very effective while given at $6 \mathrm{~h}$ after reperfusion, with a $35 \%$ decrease in infarct size. These beneficial effects were related to the reduction of nitrotyrosine generation in the ischemic brain and to the prevention of apoptotic cell death in neurons within the ischemic penumbra (1273). An additional important finding of this study was the striking reduction (70\%) of postischemic brain edema afforded by FeTPPS and FeTMPyP, pointing to an important role of peroxynitrite in the rupture of the blood-brain barrier in the ischemic brain. Such observations are consistent with the recent findings that peroxynitrite injures cerebral microvessels and increases vascular permeability in brain by mechanisms involving F-actin depolymerization in vascular smooth muscle (841) and the activation of MMP-9 in brain microvessels (495).

In a similar model of transient MCA occlusion in rats, Yu and co-workers (1425) evaluated the therapeutic potential of uric acid, a natural antioxidant with very effective properties at preventing nitration by peroxynitrite (notably, it does not directly scavenge peroxynitrite). Uric acid was given either $24 \mathrm{~h}$ before MCA occlusion or $1 \mathrm{~h}$ after reperfusion. In both conditions, uric acid reduced ischemic damage to cerebral cortex and striatum by $>70 \%$ and significantly improved behavioral outcome of the animals. The mechanisms underlying uric acid neuroprotection were then evaluated in an in vitro model of hippocampal cell culture exposed to excitotoxic concentrations of glutamate. Glutamate induced the formation of peroxynitrite, mainly localized within the mitochondria, and triggered delayed $(6 \mathrm{~h})$ calcium overload, mitochondrial depolarization, lipid peroxidation, and massive neuronal cell death. Peroxynitrite generation was suppressed by uric acid, which alleviated the mitochondrial damage, lipid peroxidation, and calcium overload produced by glutamate, resulting in an almost complete protection against neuronal death (1425).

Two additional compounds, NAC and ebselen, which enhance endogenous defenses against peroxynitrite, and which may also directly scavenge peroxynitrite, were also recently found to exert potent protection in experimental stroke. Cuzzocrea et al. (264) determined the neuroprotective effects of NAC in a model of global transient ischemia in gerbils, produced by a 5 -min bilateral occlusion of the common carotid arteries, followed by reperfusion for up 
to $48 \mathrm{~h}$. NAC almost suppressed nitrotyrosine formation within neurons and endothelial cells from the ischemic area. There was a concomitant reduction of lipid peroxidation, neutrophil infiltration, and brain edema, with a considerable prevention of histological damage in animals treated with NAC (264). Ebselen is a selenium-containing organic antioxidant which acts as a mimic of glutathione peroxidase and thus favors the maintenance of cellular reduced glutathione pools, which represent one of the major defense mechanisms against peroxynitrite. In rat permanent MCA occlusion, infusion of ebselen, starting $45 \mathrm{~min}$ before and maintained $4 \mathrm{~h}$ after the occlusion, reduced cerebral cortical damage by $28 \%$. This effect correlated with a significant prevention of lipid peroxidation and DNA oxidation, implying that these two events may represent key mechanisms of peroxynitrite-dependent neuronal damage in stroke (599).

Whereas peroxynitrite may directly injure neurons by inhibiting mitochondrial respiration, oxidizing membrane lipids, structural proteins and enzymes, as well as by consuming intracellular antioxidants such as GSH, evidence is growing that a major action of peroxynitrite (and other oxidants) in stroke is to injure DNA and activate PARP, a subject extensively reviewed in Reference 214. Accumulation of poly(ADP-ribose), as a marker of PARP activation, has been identified not only in animal models of transient (923) and permanent (1281) ischemia, but also in the infarcted human brain. Indeed, immunohistochemical evidence of PARP activation has been demonstrated in autopsy specimen, occurring in neural cells during the first $24 \mathrm{~h}$ after stroke, and in infiltrating macrophages between 3 days and $3 \mathrm{wk}$ after the episode of acute ischemia (794). Accumulation of poly(ADP-ribose) has also been detected in neurons after global cerebral ischemia due to cardiac arrest, located in regions of ischemic damage and immediately adjacent neocortex (793). The essential role of nNOS-derived peroxynitrite on PARP activation in stroke has been attested by the significant prevention of PARP activation provided by genetic deletion (359) or pharmacological inhibition (1281) of nNOS. The activation of PARP induces major neurotoxicity, as evidenced by the extreme protection against brain damage in PARP knockout mice subjected to transient MCA occlusion (up to $80 \%$ reduction of infarct volume) $(349,360)$. A similar high degree of protection could be reproduced by a series of pharmacological inhibitors of PARP, such as 3-aminobenzamide (360), PJ-34 (1), or INO-1001 (624). Importantly, these agents remained effective even when given several hours after the ischemic insult, which indicates that these drugs might be clinically useful. Regarding mechanisms, PARP-dependent neurotoxicity appears multifactorial, involving the depletion of NAD/ATP leading to neuronal necrosis (360), the upregulation of transcription factors ( $\mathrm{AP}-1, \mathrm{NF} \kappa \mathrm{B})$ enhancing postischemic brain inflammation (697), and the activation of AIF (see sect. IV), activating caspase-independent apoptosis (294).

2. Other forms of reperfusion injury-Although most experimental effort investigating NO and peroxynitrite's actions in reperfusion injury has so far been focused on the brain and heart (see above and sect. VIA), evidence also exists that theses species are involved in other reperfusion paradigms. In such conditions, the mechanisms underlying the formation of NO, superoxide, and peroxynitrite, as well as their cellular targets, are similar to those exposed in detail in the sections on stroke and myocardial I/R. Therefore, here we only provide a brief summary of the major relevant studies implying NO/peroxynitrite generation as key players of reperfusion injury in organs such as the gut, the liver, the lung, and the kidney.

A) KIDNEY: As in many other conditions, NO plays both beneficial and detrimental effects in kidney I/R (KIR), depending on the rate and timing of its formation as well as the isoform from which it is produced as extensively reviewed by Goligorsky et al. (454). Crucial in vitro experiments demonstrated that proximal renal tubules isolated from mice with a targeted deletion of iNOS were resistant to hypoxia, contrasting with the lethal damage produced by the same degree of hypoxia in tubules prepared from mice lacking eNOS or nNOS (774). In 
vivo, iNOS inactivation by selective iNOS pharmacological inhibitors $(200,1429)$, or iNOS antisense oligodeoxynucleotides (948), protected kidneys from KIR injury, while nonselective NOS inhibitors did not provide protection (454). Importantly, evidence for peroxynitrite generation in injured tubules has been reported in KIR, where injury was reduced by anti-iNOS strategies (200). It has also been shown that an NO donor given before the induction of ischemia reduced nitrotyrosine and exerted beneficial influence, while it enhanced nitrotyrosine formation and increased renal damage when given $6 \mathrm{~h}$ after reperfusion (928). These data all point to a protecting role of eNOS-dependent NO formation in the early phase of KIR, and a detrimental action of iNOS-derived NO and peroxynitrite at later stages (for review, see Ref. 454). The latter assertion has been confirmed by a series of studies showing that strategies directly preventing peroxynitrite generation or accelerating its degradation prevented the generation of nitrotyrosine in injured tubular cells, ameliorated renal histological damage, and reduced functional renal alterations (evaluated by the increase of plasma creatinine and urea, and the development of proteinuria) in KIR. Strategies aimed at limiting peroxynitrite in these studies involved uric acid (1418), MnTBAP (1429), and various compounds extracted from plants with documented peroxynitrite scavenging activity in vitro, e.g., (-)-epicatechin 3-Ogallate (1418), Coptidis Rhizoma (1417), and sanguiin H-6 (1416).

B) GUT: An abundant literature has implicated NO in the pathophysiology of gut I/R (GIR) injury. Basal NO production dependent on constitutive NOS (nNOS or eNOS) is essential in minimizing mucosal and microvascular barrier dysfunction associated with reperfusion of the postischemic intestine (717). Several NO donors have thus been shown to exert beneficial effects on mucosal and microvascular function in this setting $(648,831)$. Alternatively, increased NO production due to the expression of iNOS in the intestinal mucosa is detrimental to intestinal integrity: mice with genetic deletion of iNOS exhibited resistance against reperfusion-induced mucosal injury and bacterial translocation (1220), whereas administration of selective iNOS inhibitors such as aminoguanidine (787) and ONO-1714 (926) prevented the hemodynamic collapse, intestinal microcirculatory derangements, lipid peroxidation, and neutrophil infiltration in rat models of GIR. There is additional evidence implicating the formation of free radicals, most notably superoxide, as a critical mechanism of intestinal damage and dysfunction related to ischemia. Thus both the SOD mimetics M40401 (267) and the broad-spectrum antioxidant tempol $(270,271,273)$ attenuated gut inflammation and damage in rodents.

There are many indications that peroxynitrite generation is the ultimate mechanisms underlying $\mathrm{NO}$ and $\mathrm{O}_{2}{ }^{--}$toxicity in GIR. Massive nitrotyrosine staining is evident in the ileum of rats exposed to GIR and can be reduced by strategies blocking either the formation of NO (274) or superoxide (267). In addition, the peroxynitrite decomposition catalyst FeTMPS reversed tissue injury, reduced lipid peroxidation, and alleviated neutrophil infiltration in the reperfused bowel (272). The latter effect was associated with an attenuation of reperfusion-induced upregulation of the adhesion molecules P-selectin and intracellular adhesion molecule-1. Similar results were obtained when peroxynitrite generation was inhibited with NAC (263) or melatonin (260). Finally, experiments performed either with pharmacological inhibitors or genetic suppression of PARP showed an almost complete protection against the development of mesenteric barrier failure complicating $\operatorname{GIR}(277,768)$. These different studies support the contention that peroxynitrite formation due to $\mathrm{NO}$ and superoxide overproduction in the reperfused intestine induces gut damage and inflammation, notably by stimulating the mechanisms recruiting activated phagocytes, and also results in gut barrier failure by triggering the activation of PARP within the gut mucosa.

C) LIVER: Liver I/R (LIR) is a significant clinical problem involved in the liver failure associated with circulatory shock, vascular exclusion during surgery, and liver transplantation. An important early mechanism of liver failure in such conditions is the marked sinusoid 
vasoconstriction that develops during reperfusion, as a consequence of an imbalance between vasoconstrictors, especially endothelins, and vasodilators, essentially NO (621). In the liver, NO is normally produced exclusively by eNOS expressed by sinusoid endothelial cells (207). In LIR, a significant reduction of eNOS activity has been demonstrated both in humans (1319) and animals (1146), precipitating sinusoidal vasoconstriction (621). These microcirculatory disturbances can be alleviated by the administration of NO donors (335, 722), while they are seriously aggravated by the administration of nonselective NOS inhibitors (904) or by eNOS gene deletion (660), which have been associated with significant enhancement of LIR-induced liver injury. LIR also rapidly activates Kupffer cells, which then produce proinflammatory cytokines, free radicals, oxidants, and large amounts of NO due to iNOS expression $(207,621)$. These events result in the activation of neutrophils, endothelial cells, and hepatocytes, which further release toxic levels of oxidant species and iNOS-derived NO in the delayed phase of LIR $(207,621)$. The pathogenic role of oxygen-centered radicals in this setting has been well established by the significant reduction of liver damage provided by various antioxidant strategies $(1361,1400)$.

Despite the increased generation of $\mathrm{NO}$ and the ensuing formation of peroxynitrite, evidenced by nitrotyrosine formation in LIR $(1146,1319)$, the pathogenic role of these abnormalities remains largely debated. On the one hand, selective iNOS inhibitors such as FK330 (1295) and ONO-1714 $(863,1257)$ reduced nitrotyrosine formation and ameliorated liver injury and dysfunction by reducing leukocyte trafficking, cytokine expression, as well as hepatic apoptosis and necrosis. One study in iNOS-deficient mice also indicated that these mice experienced a slight protection against LIR (739). On the other hand, studies also revealed that selective iNOS inhibitors could impair hepatic blood flow and increase liver injury, suggesting that iNOS, in a way similar to eNOS, exerts an essential role in maintaining liver sinusoidal perfusion in LIR $(570,1347)$. Furthermore, a significant aggravation of LIR-mediated damage has been documented in a second study performed in iNOS knockout mice (548), although the relevance of these findings is questionable, given the absence of iNOS expression in wild-type control mice in this particular study.

It has been suggested that any peroxynitrite formed during LIR would be effectively scavenged by the high intrahepatic stores of reduced glutathione, which would thereby mitigate any potential toxic effects of peroxynitrite (621). It is also worth noting that peroxynitrite might even exert beneficial effects in LIR, as proposed by Liu et al. (782). In a model of rat LIR, the exogeneous administration of $2 \mu \mathrm{mol} / \mathrm{kg}$ peroxynitrite at 0,60 , and $120 \mathrm{~min}$ postreperfusion reduced neutrophil accumulation and liver damage (782). Clearly, the studies summarized above have highlighted the controversial roles of NO and peroxynitrite in LIR. Further studies using well-designed selective iNOS inhibitors and peroxynitrite decomposition catalysts should be performed to gain more precise insights into their actions in this condition.

D) LUNG: Lung I/R injury (LIRI) develops in as much as $25 \%$ of patients after lung transplantation and is a major cause of primary graft dysfunction and early postoperative mortality. The donor alveolar macrophages appear to play a critical role in the initiation of lung damage upon reperfusion, by secreting inflammatory cytokines, resulting in the secondary recruitment of recipient activated neutrophils, with ensuing inflammation, epithelial and endothelial damage leading to reperfusion lung edema (397). The role of NO in LIRI is unclear at present. Many animal studies have reported beneficial effects of inhaled NO therapy in models of LIRI induced by transient pulmonary artery occlusion, or by lung transplantation. In pigs, short pretreatment with inhaled NO (15-20 ppm for 10-30 min) either before lung ischemia (1338) or before lung harvest in the donor animal (455), significantly reduced reperfusion pulmonary damage, via mechanisms involving reduced IL-8 production and decreased neutrophil infiltration (455), as well as a reduced formation of free radicals (1338). Comparable beneficial effects were reported in rabbit models of LIRI and lung transplantation, 
where inhaled NO was found to improve endothelial integrity and to reduce apoptotic cell death of pulmonary cells $(1139,1406)$. There are further indications that intravenous administration of nitroglycerin can alleviate lung damage induced by LIRI in pigs and rats $(341,788)$. In humans, a recent prospective study has evaluated inhaled NO therapy ( $20 \mathrm{ppm}$, initiated 10 min after reperfusion) in lung transplant recipients (862). Unfortunately, this strategy did not confer significant protection, although it tended to reduce the duration of mechanical ventilation. This negative study has however been criticized as being largely underpowered to detect any significant effects of inhaled NO.

At variance with the above-mentioned results, studies demonstrating cytotoxic roles of NO in LIRI have also been presented. Schutte et al. (1138) showed that the NOS inhibitor L-NMMA protected against lung damage induced by reperfusion, only if the ischemic phase was accompanied by hypoxic, but not normoxic, ventilation. In this condition, a surge of NO production occurred in early reperfusion, which was abrogated by L-NMMA. Furthermore, SOD also produced significant protection, suggesting an essential role of superoxide and NOderived peroxynitrite formation in mediating lung damage in LIRI (1138). In line with these findings, LIRI has been associated with significant expression of iNOS (977) and with the abundant formation of nitrotyrosine in lung tissue $(607,977)$, and a selective iNOS inhibitor, $1400 \mathrm{~W}$, reduced reperfusion-induced lung damage in a model of LIRI in the rabbit (977).

Finally, accelerating the decomposition of peroxynitrite with FP- 15 was recently found to exert major benefits in rats exposed to $90 \mathrm{~min}$ of lung ischemia/anoxia followed by $60 \mathrm{~min}$ of reperfusion (925). FP-15 reduced nitrotyrosine formation and the expression of the chemokines MIP-1 $\alpha$, CINC, and MIP-2. It also limited neutrophil infiltration, lung edema, and significantly ameliorated histological changes $(924,925)$.

In summary, $\mathrm{I} / \mathrm{R}$ is a crucial pathophysiological mechanism of organ damage in many different clinical conditions, including myocardial infarction, stroke, circulatory shock, surgery, or organ transplantation. In most of these instances, a dual role of NO, protective and deleterious, has been established. Protection has generally been attributed to the actions of eNOS-derived $\mathrm{NO}$, due to its vasodilating, anti-inflammatory, and antithrombotic effects. In contrast, enhanced NO production consecutive to iNOS induction (and, in the brain, to the stimulated activity of nNOS), has been associated with detrimental consequences, mostly related to the secondary generation of peroxynitrite. In line with these observations, ample experimental evidence shows significant benefit of therapeutic strategies aimed at manipulating peroxynitrite formation/degradation in the pathological paradigm of I/R.

\section{G. Nitric Oxide and Peroxynitrite in Neurodegenerative Disorders}

NO is produced by all brain cells including neurons, endothelial cells, and glial cells (astrocytes, oligodendrocytes, and microglia) by $\mathrm{Ca}^{2+} /$ calmodulin-dependent NOS isoforms.

Physiologically NOS in neurons (nNOS, type I NOS) and endothelial cells (eNOS, type III NOS) produce nanomolar amounts of NO for short periods in response to transient increases in intracellular $\mathrm{Ca}^{2+}$, which is essential for the control of cerebral blood flow and neurotransmission and is involved in synaptic plasticity, modulation of neuroendocrine functions, memory formation, and behavioral activity $(491,890,1229)$. The brain produces more NO for signal transduction than the rest of the body combined, and its synthesis is induced by excitatory stimuli. Consequently, NO plays an important role in amplifying toxicity in the CNS. Indeed, under various pathological conditions associated with inflammation (e.g., neurodegenerative disorders and cerebral ischemia), large amounts of $\mathrm{NO}$ are produced in the brain as a result of the induced expression of iNOS (type II NOS) in glial cells, phagocytes, and vascular cells, which can exert various deleterious roles $(39,491,890)$. Thus NO may be a double-edged sword, exerting protective effects by modulating numerous physiological processes and complex immunological functions in the CNS on one hand and on the other hand 
mediating tissue damage $(446,491,890)$. The detailed discussion of the role of NO in the pathophysiology of various neurodegenerative disorders including Parkinson's disease, Alzheimer's disease, Huntington's disease, multiple sclerosis (MS), and amyotrophic lateral sclerosis (ALS), just mentioning a few, is the subject of numerous excellent recent overviews $(77,145,194,219,491,890,1003,1205,1433)$ and beyond the scope of this paper. Here we cover only the role of peroxynitrite and protein nitration, which are likely responsible for most deleterious effects of NO in neurodegenerative disorders.

A common feature of neurodegenerative diseases, such as Parkinson's disease, Alzheimer's disease, MS, and ALS is extensive evidence of oxidative stress, which might be responsible for the neuronal dysfunction and death contributing to pathogenesis of these disorders (reviewed in Refs. 20,33,59,63,173,608,686,900,901,1037,1038,1251). Because neurons are incapable of division, the consequences of severe oxidative stress killing neurons makes sustained damage irreversible. Yet, it is important to look beyond damage to neurons as oxidants may also have more subtle roles in compromising the integrity of the blood-brain barrier and in producing reactive changes in astrocytes. Oxidants induce distinct pathological consequences that amplify and propagate injury and eventually cause irreversible degeneration. Moreover, oxidative stress appears to provide a critical link between environmental factors such as exposure to pesticides, herbicides, and heavy metals with endogenous and genetic risk factors in the pathogenic mechanisms of neurodegeneration, particularly in Parkinson's disease $(63,608,632)$. The discovery that 1 -methyl-4-phenyl-1,2,3,6-tetrahydropyridine (MPTP) toxicity leads to Parkinson's-like disease provided strong evidence that complex I within mitochondria may be a major source of superoxide that initiates the disease (1043). More recently, paraquat, a commonly used pesticide and a well-known inhibitor of complex I, has also been identified as a potential cause of Parkinson's disease $(700,1114)$. Both of these compounds are capable of transferring electrons from complex I to oxygen, producing superoxide (419). Simultaneously increased NO (see above) and oxidative superoxide production associated with neurodegenerative disorders favors the in vivo generation of peroxynitrite $(608,1119,1284)$.

Peroxynitrite formation has been implicated in Alzheimer's disease, Parkinson's disease, Huntington's disease, MS, ALS, and traumatic brain injury (reviewed in Refs. 194,608,1119, 1284). Nitrotyrosine immunoreactivity has been found in early stages of all of these diseases in human autopsy samples as well as in experimental animal models. Increased nitrite, nitrate, and free nitrotyrosine has been found to be present in the cerebral spinal fluid (CSF) and proposed to be useful marker of neurodegeneration (168; reviewed in Refs. 608,1119,1284). Once formed in the diseased brain, peroxynitrite may exert its toxic effects through multiple mechanisms, including lipid peroxidation, mitochondrial damage, protein nitration and oxidation, depletion of antioxidant reserves (especially glutathione), activation or inhibition of various signaling pathways, and DNA damage followed by the activation of the nuclear enzyme PARP $(608,1119,1284)$.

Tyrosine nitration represents a major cytotoxic pathway in the nervous system, possibly contributing to neurodegenerative disorders. Mitochondria are particularly vulnerable targets of oxidative stress and protein nitration in neurodegeneration (1135). Increased superoxide production in mitochondria should render them vulnerable when exposed to NO (532). Consistently, mice deficient in NOS1 are resistant to MPTP toxicity, and NOS inhibitors are protective (1348). Peroxynitrite also readily damages complex I in the mitochondria (919, 1062) and might further amplify injury. One of the major consequences of peroxynitrite production within mitochondria is nitration and inactivation of mitochondrial Mn-SOD (826, 827,830). In vivo, nitration of Mn-SOD has been detected in cerebrospinal fluid of patients with ALS, Alzheimer's disease, and Parkinson's disease (27). Serious consequences arise from the genetic knockout of Mn-SOD, which is generally lethal in the neonatal period. In addition 
to causing cardiac failure, the mitochondrial Mn-SOD knockout mouse suffers CNS pathology that includes mitochondrial vacuolization and oxidized lipid deposits. Conversely, genetically induced increased expression of mitochondrial Mn-SOD or induction of the enzyme during stress has been shown to protect mitochondria and cells from oxidative stress. The inactivation of Mn-SOD by peroxynitrite will make mitochondria more vulnerable in neurodegeneration.

Another major group of targets for nitration in neurodegeneration are various structural proteins. These are particularly abundant proteins in cells and tend to have a higher proportion of tyrosine. Tyrosine is moderately hydrophobic and can be buried within the contact regions between subunits and yet also can form hydrogen bonds with water when subunits are disassembled. Often these tyrosines are located near acidic and basic residues that can increase their susceptibility to nitration (72). Nitration of structural proteins can have major functional consequences. Addition of the bulky nitro group will increase steric interference and introduce a partial negative charge. If a tyrosine involved in subunit contacts is nitrated, it could seriously compromise the formation of a large structure. The nitration of the structural protein has a dominant effect interfering with the proper assembly and function of the entire structure.

Tyrosine radical intermediates may also cause covalent cross-links between structural proteins that will also interfere in their proper assembly and function. Disruption of structural proteins might also have a major impact on mitochondrial function in neurons, because mitochondria moving along axons are far removed from nuclear protein synthesis (2). Over $99 \%$ of the proteins in the mitochondria are encoded in the nucleus and imported. In neurons, mitochondria could potentially be several days to months away from the cell soma, making replacement of key components difficult to maintain.

The CNS intermediate filament proteins, neurofilament-L (NFL) and glial fibrillary acid protein (GFAP) were among the very first proteins to be identified as endogenously nitrated in vivo $(247,459,531)$. SOD was also shown to be very efficient at catalyzing nitration by peroxynitrite of disassembled NFL subunits in vitro, particularly when the SOD is zinc deficient $(246,247)$. The most likely sequences to be nitrated were in the coiled-coil rod domain that is crucial for the initial dimerization, but not in the head domain where 10 of its 100 residues are tyrosine. Nitration of a small percentage $(<10 \%)$ of NFL is sufficient to substantially disrupt the assembly of neurofilaments. The nitration of NFL may be particularly important in ALS where motor neurons contain massive concentrations of neurofilament proteins needed to support the enormous axons that can be a meter in length. Over $99 \%$ of the volume of motor neurons is contained in the axon plus the neuromuscular junction and hyperphosphorylated neurofilaments found in inclusions are an early marker of motor neuron pathology in ALS. Neurofilament proteins also have a high affinity and binding capacity for zinc (1028), which may favor the formation of zinc-deficient SOD. As described below, the loss of zinc from SOD may be a key factor in understanding why motor neurons are vulnerable in ALS.

Subsequent studies have found numerous other proteins that may lead to aberrant structures when nitrated. Mutations to $\alpha$-synuclein have been linked to a small fraction of Parkinson's cases. Wild-type $\alpha$-synuclein is a major component in Lewy bodies as well as other filamentous protein inclusions in sporadic PD and several other pathologies $(22,443,1042,1191)$. The use of specific antibodies for nitrated $\alpha$-synuclein revealed that the majority of the Lewy bodies and protein inclusions contain nitrated and oxidized $\alpha$-synuclein (443). Cellular systems and biochemical studies have shown that nitrative stress promotes the formation of $\alpha$-synuclein aggregates in a manner that is resistant to proteolysis $(608,1191,1288)$. Nitration of other structural proteins such as synaptic proteins (synaptophysin, Munc-18, SNAP25) (317), Tau protein $(1073,1074,1442)$, and tubulin $(54,199,730,1269$; see also Table 1) may also be involved in the pathophysiology of various neurodegenerative disorders and stroke. 
1. Multiple sclerosis (MS)-MS is an immune-mediated, inflammatory disease causing focal damage to the white matter attacking different regions of the CNS apparently at random. The ability of neurons to conduct impulses becomes compromised through the loss of their myelin sheath, and through the secondary axonal loss (1187). The disease affects $2-3$ million people worldwide and most commonly shows an unpredictable, relapsing-remitting course and a range of clinical symptoms (e.g., tremor, ataxia, visual loss, double vision, weakness or paralysis, difficulty in speaking, loss of bladder control and constipation, cognitive impairment, and painful muscle spasms) depending on where the demyelination and axonal loss have occurred (226). Although numerous drugs targeting the immune system slow down the progression of the disease, the treatment of MS remains mostly symptomatic and far from satisfactory $(675,1036)$.

Inflammatory plaques in MS patients showed increase immunoreactivity for iNOS and nitrotyrosine $(41,123,139,241,242,445,546,780,1154,1312)$ accompanied by elevated levels of nitrate and nitrite in CSF, urine, and serum $(447,448,1407)$. Tyrosine nitration may contribute to the blood-brain barrier damage (allowing the influx of inflammatory cells) which is a cardinal feature of active MS $(296,567,972)$. Furthermore, iNOS expression decreases in demyelinated plaques from MS patients when inflammation is reduced (780). Inhibition of iNOS in experimental allergic autoimmune encephalomyelitis (EAE; mouse model of MS) yielded controversial results, since both reduction $(242,1142)$ or enhancement $(644)$ of inflammation were reported. Peroxynitrite scavengers, mercaptoethylguanidine (MEG) and guanidinoethyldisulphide (GED), protected only against the induction phase of EAE, but did not prevent disease progression (1142).

Uric acid has proven to be a useful inhibitor of tyrosine nitration (although it is not a direct peroxynitrite scavenger) (1271) and has been shown to protect the blood-brain barrier and largely prevent the entry of inflammatory cells into the CNS $(566,567)$. Additionaly, it also prevented CNS injury after inflammatory cells have already migrated into the CNS (1141). Urate has also proven beneficial in reducing the morbidity associated with viral infections $(710,1141)$. Interestingly, in humans there is an inverse correlation between affliction with gout and MS $(710,1195)$. Numerous studies have reported lower levels of uric acid in MS patients favoring the view that reduced uric acid in MS is secondary to its "peroxynitrite scavenging" activity during inflammatory disease, rather than a primary deficiency (reviewed in Ref. 694). These studies have also suggested that serum uric acid levels could be used as biomarkers for monitoring disease activity in MS (reviewed in Ref. 694).

2. Parkinson's disease-Parkinson's disease (PD), the second most common neurodegenerative disease of adult onset, is characterized by progressive loss of dopaminergic neurons within substantia nigra pars reticulata $(\mathrm{SNr})$, resulting in reduced dopamine levels and a loss of dopaminergic neurotransmission in the striatum, which interferes with the function of the basal ganglia critical to motor function and coordination. Even though oxidative stress, inflammation, excitotoxicity, mitochondrial dysfunction, and hereditary and environmental factors have all been implicated in the pathogenesis of PD, the exact cause of the loss of dopaminergic neurons remains obscure $(362,522)$.

Post mortem studies have demonstrated ROS- and peroxynitrite-mediated oxidative and/or nitrosative damage in PD (reviewed in Refs. 337,338,632,1119,1284). Increased nitrotyrosine accumulation has been found in Lewy bodies (characteristic feature of PD; Ref. 456) and polymorphonuclear cells of PD patients (437). In the latter, the accumulation of nitrotyrosinecontaining proteins was accompanied by overexpression of nNOS (437). In a mouse model of PD induced by intraperitoneal administration of a selective neurotoxin of dopaminergic nigrostriatal neurons MPTP elevated levels of both free and bound 3-NT were demonstrated in ventral midbrain and in the striatum (1020). Consistent with the pathogenetic role of NO in 
$\mathrm{PD}, \mathrm{nNOS}$ knockout mice or mice or primates treated with nNOS inhibitors are more resistant to neurotoxicity induced by MPTP $(68,1044)$.

Peroxynitrite may induce nitration of tyrosine hydroxylase, the initial and rate-limiting enzyme in the biosynthesis of dopamine, leading to inhibition of enzyme activity and consequent failure in the synthesis of dopamine (28). Importantly, the nitration of tyrosine residues in tyrosine hydroxylase paralleled the decline in dopamine levels in mouse striatum following MTPT administration (28). Tyrosine residues are also pivotal for the substrate specificity of monoamino oxidase B (MAO B) leading to impaired dopamine metabolism once nitrated (440). Peroxynitrite has been implicated to contribute to the loss of intracellular glutathione from substantia nigra (an early event in PD) by inactivating glutathione reductase, the enzyme responsible for the regeneration of glutathione from its oxidized form $(60,97)$, and to induce apoptosis in dopaminergic neurons in PD (930). Recent evidence suggests that mitochondrial complex I inhibition may be the central cause of sporadic PD and that derangements in complex I lead to $\alpha$-synuclein aggregation, which contributes to the demise of dopamine neurons (293). Accumulation and aggregation of $\alpha$-synuclein may further facilitate the death of dopamine neurons through impairments in protein handling and detoxification (293). As already mentioned above, both mitochondrial complex I and synuclein can be targets for peroxynitrite-induced protein nitration (see also Table 1).

3. Alzheimer's disease-Alzheimer's disease (AD), a progressive neurodegenerative disorder, is characterized by the formation of neuritic plaques rich in $\beta$-amyloid $(\mathrm{A} \beta)$ peptide, neurofibrillary tangles rich in hyperphosphorylated tau protein, gliosis, and a neuroinflammatory response involving astrocytes and microglia, inevitably leading to progressive global cognitive decline, and accounting for the vast majority of age-related dementia (1357).

Increased oxidative stress is present and appears to be an early event in the process of neurodegeneration associated with $\mathrm{AD}(162,356,564,845,872,900,1167,1278)$. It appears that both neuronal and glial NOS may play a role in the pathogenesis of AD and peroxynitrite formation. Increased expression of nNOS was reported in neurons with neurofibrillary tangles in the hippocampus and enthorinal cortex of $\mathrm{AD}$ patients as well as in reactive astrocytes near amyloid plaques $(1166,1276)$. Presence of neuritic plaques has also been associated with increased expression of iNOS and eNOS in astrocytes $(297,298,804-806,1340)$.

Dysfunctional neurons and neurofibrillar tangles frequently display various markers of nitrative stress, including 3-NT, the hallmark of peroxynitrite formation. Indeed, increased nitrotyrosine levels were demonstrated in hippocampus, neocortical regions, and ventricular fluid and CSF of AD patients $(538,1176,1279)$. Importantly, the high levels of 3-NT in cerebrospinal fluid correlated with the decrease in cognitive function in AD patients (1279). In contrast, a study found no difference in 3-NT levels in CSF of patients with AD (1095). Immunohistochemical staining for 3-NT is more intense around $\mathrm{A} \beta$ depositions, and chronic intracerebroventricular infusion of $\mathrm{A} \beta$ can also cause ROS and peroxynitrite formation and subsequent tyrosine nitration of proteins $(1119,1285)$. Nitration of a variety of proteins including synaptophysin, proteins involved in glucose metabolism, cytoskeletal integrity, antioxidant defense, just naming a few, was also reported in $\mathrm{AD}(180,225,1285)$.

4. Amyotrophic lateral sclerosis (ALS)_ALS (also known as Lou Gehrig's disease or motor neuron disease) is the most common adult-onset human motor neuron disease, which is characterized by rapid, progressive degeneration of motor neurons in the motor cortex, brain stem, and spinal cord. This ultimately leads to progressive weakness, paralysis, and premature death within $3-5$ years after the onset of symptoms $(152,1088)$. Patients are cognitively intact and thus completely aware of their progressive disability. Only $\sim 10 \%$ has familial inheritance, 
usually with an autosomal dominant pattern, while most ALS cases are sporadic with no genetic basis. Although various mechanisms, including neuroinflammation, oxidative stress, autoimmunity, a defect in neuronal glutamate transport and glutamate toxicity, neurofilament accumulation, exogenous factors (viruses, toxins), mitochondrial dysfunction, and mutations in the SOD gene were implicated to play a role, the pathogenesis of ALS is incompletely understood $(63,91,152,872,1088,1168,1309,1359)$. Tragically, available treatment options do not prevent disease progression and death and extend life by only a few weeks (1088).

In 1993, an international collaboration identified 13 missense mutations to SOD in $\sim 2-3 \%$ of ALS patients (1086). Further screening has identified over 120 different missense mutations occurring at about 45 different places that are linked to ALS (a current list is maintained at www.alsod.org). The disease caused by the SOD mutations is generally not clinically or pathologically distinguishable from the sporadic cases of ALS. The mutations to SOD are dominant, and almost all the mutants result in the expression of an active SOD protein. Overexpression of many but not all of the ALS mutations in transgenic mice or rats results in the development of motor neuron disease, which strongly supports a toxic gain of function in SOD induced by the mutations (493). The disease is clearly not the result of losing SOD scavenging activity, because knockout mice for this gene do not develop motor neuron disease (1070).

Because SOD could catalyze tyrosine nitration by peroxynitrite, Beckman et al. (76) proposed that the mutations may result in greater nitration activity. A number of investigators have since found increased tyrosine nitration in both human ALS patients $(3,4,69,217,218,457,1280$, 1384 ) and in transgenic mouse model of familial ALS (animals overexpressing SOD mutants) $(151,176,388,1121,1135)$. More recently, the nitration of proteins in transgenic mice has been directly confirmed using mass spectrometry (176). Protein nitration may have a role in ALS pathogenesis, acting directly by inhibiting the function of specific proteins and indirectly interfering with protein degradation pathways and phosphorylation cascades (176).

However, when the SOD proteins were purified and carefully treated to have $100 \%$ of their complement of copper and zinc, the ALS mutant SOD proteins behaved identically to wildtype $\mathrm{Cu}, \mathrm{Zn}-\mathrm{SOD}$, reacting to peroxynitrite with the same efficiency and with equal superoxide scavenging activity. However, the proteins were slightly less stable than wild-type SOD and were often deficient in zinc $(246,247)$. When the SOD proteins were made zinc deficient, they had decreased scavenging activity for superoxide and reacted more efficiently with peroxynitrite to catalyze tyrosine nitration. In addition, the copper remaining in SOD was much more redox active and quickly reacted with ascorbate or other low-molecular-weight reductants typically found within cells. This aberrant redox activity will deplete cells of their antioxidant defenses. In addition, the copper- and zinc-deficient SOD will be entirely reduced, continuously reacting with oxygen to generate superoxide. In the presence of NO, zincdeficient SOD will produce peroxynitrite as well as increase tyrosine nitration. The loss of zinc turns SOD into a strongly pro-oxidative enzyme (reviewed in Refs. 77,608).

Of particular importance, the loss of zinc from wild-type SOD carries the same functional consequences as the loss of zinc from any of the ALS mutant SODs. The mutations simply increased the probability of SOD lacking the essential zinc atom by destabilizing the structure of SOD. As a consequence, the loss of zinc from SOD could be involved in the pathology of the $98 \%$ of ALS patients who do not have mutations to the SOD protein.

The toxicity of zinc-deficient SOD to primary cultures of motor neurons was confirmed in vitro by entrapping these proteins into liposomes to allow intracellular delivery (368). The zincdeficient wild-type SOD was as toxic as any of eight different zinc-deficient ALS mutant SODs (366). The toxicity of all the zinc-deficient SODs was inhibited by copper chelators or by 
inhibition of NO synthesis. Exposure of motor neurons to exogenous NO itself was not toxic but overcame the protection provided by NOS inhibitors. Zinc-deficient SOD also increased tyrosine nitration in the motor neurons before inducing cell death. In contrast, $\mathrm{Cu}, \mathrm{Zn}$ ALSmutant SODs were not toxic to motor neurons and provided equal protection as $\mathrm{Cu}, \mathrm{Zn}$ wildtype SOD to motor neurons subjected to trophic factor deprivation. Hence, the loss of zinc from SOD provides a compelling hypothesis to explain how mutations to this common "housekeeping" enzyme can cause the selective death of motor neurons in ALS (77).

Trophic factor deprivation of primary cultures of motor neurons initiates the endogenous production of peroxynitrite, which in turn activates apoptosis $(369,371)$. The mechanism appears to be specific for motor neurons and involves a novel pathway with the activation of FAS and induction of nNOS (1068). This pathway is readily induced in primary cultures of motor neurons isolated from transgenic mice overexpressing mutant SOD but only if they are exposed to an exogenous source of NO (1068). Trophic factor deprivation and other stresses to motor neurons apparently activate an intracellular source of superoxide, which activates apoptosis when NO is also coexpressed $(366,369)$.

Peroxynitrite can participate in the pathogenesis of ALS, because it can activate spinal cord astrocytes, which normally provide excellent trophic support to motor neurons, to assume a reactive phenotype that induces the death of motor neurons (179). The original methods for culture of motor neurons were to grow them on a feeder layer of astrocytes. A variety of cytokines as well as LPS causes astrocytes to produce peroxynitrite and assume the reactive phenotype (179). The reactive phenotype increases the production of nerve growth factor (NGF) $(1018,1322)$. Normally NGF has no effect on motor neurons. However, motor neurons in culture as well as in ALS induce the expression of p75, which is a low-affinity receptor for neurotrophins such as NGF. When motor neurons express p75, they become sensitive to NGF when an exogenous source of NO is added. Reactive astrocytes synthesize and release both NGF and NO in quantities sufficient to induce the death of motor neurons.

The significance of this intricate interplay may have important ramifications not only for ALS but also for PD and AD $(6,58,1102)$. Reactive astrocytes are common hallmark of neurodegeneration, and these results suggest that peroxynitrite may play an important role in promoting this phenotype as well as causing the degeneration of neurons. In ALS, the transformation of astrocytes into a reactive phenotype may explain why ALS is progressive, causing the relentless death of neighboring motor neurons. Interfering in such a cascade to stop the progressive death of motor neurons would not necessarily cure ALS but may keep it from being a death sentence.

5. Huntington's disease-Huntington's disease (HD) is a progressive, autosomal dominant, neuropsychiatric disorder of the midlife, caused by an unstable expansion of a trinucleotide polyglutamine repeat in the $\mathrm{NH}_{2}$-terminal domain of a protein termed huntingtin on chromosome 4 . The disorder is characterized by degeneration of neurons in the basal ganglia and cortical regions, which results in motor disturbances, such as chorea (involuntary movements) and dystonia, psychiatric symptoms, and dementia $(21,431,866)$. The prevalence of HD is much lower than that of most of the other common neurodegenerative disorders discussed above. The therapy of HD is very limited and mostly symptomatic $(21,431,866)$.

There is accumulating evidence suggesting that increased oxidative stress and excessive production of NO might contribute to the development of HD by damaging neighboring neurons (reviewed in Refs. 63,163). Accordingly, increased iNOS expression was observed in neuronal, glial, and vascular cells from brains of HD patients and mouse models of disease $(206,491)$. Similarly, numerous studies have demonstrated increased 3-NT formation in brain tissues (neurons, glia, and/or vasculature) of mice transgenic for the HD mutation, rats injected 
into the striatum with quinolinic acid (rat model of HD), and HD patients (300-302,427, $1022,1023,1096,1117)$. Importantly, both NOS inhibitors and peroxynitrite scavengers decreased neuronal damage, improved disease progression, and also decreased brain 3-NT content in experimental models $(301,1022,1117)$. These results suggest that peroxynitrite might be an important mediator of oxidative damage associated with HD.

6. Traumatic brain injury-Traumatic brain injury (TBI), which is characterized by axonal and neuronal cell injury, cerebral edema, increased permeability of blood-brain barrier, and posttraumatic changes in cognitive and neurological function, is one of the leading causes of disability and mortality in young individuals, with very limited treatment options $(67,376$, $563,817)$. TBI may trigger multiple pathophysiological processes including glutamate-induced excito-toxicity, release of inflammatory cytokines from brain-resident cells (microglia, neurons, and astrocytes), cortical blood flow dysregulation, oxidative and nitrosative stress, and eventually cell death via apoptosis or necrosis $(441,1346)$.

The pathogenetic role of peroxynitrite in TBI is supported by evidence demonstrating increased brain 3-NT levels following TBI in experimental mouse and rat models (92-94,423,507,508, $898,1171,1360)$, and by the beneficial effects of NOS inhibitor and peroxynitrite scavengers in reducing neuronal injury and improving neurological recovery following injury $(423,508$, 898).

Collectively, multiple lines of evidence discussed above provide strong support for the important role of peroxynitrite formation and/or protein nitration in neurodegenerative disorders and suggest that the neutralization of this reactive species may offer significant therapeutic benefits in patients suffering from these devastating diseases.

\section{H. Nitric Oxide and Peroxynitrite in Diabetes and Diabetic Complications}

Diabetes mellitus is one of the most costly chronic diseases with an estimated worldwide prevalence of 170 million in 2002, which is expected to double by 2030 according to the World Health Organization (1370). Type 2 diabetes, accounting for $90 \%$ of diabetes, is characterized by insulin resistance and often is associated with obesity and dyslipidemia. Type 1 diabetes occurring predominantly in children and young adults, accounting for 5-10\% of diagnosed cases, is characterized by insulin deficiency as a result of destruction of insulin-producing $\beta$ islet cells in the pancreas. Hyperglycemia is the most prominent feature of both types of diabetes. The majority of diabetic complications are associated with pathological alterations in the vascular wall, and the macro- and microvascular diseases are the most common causes of morbidity and mortality in patients with diabetes mellitus (the global mortality attributed to diabetes is $6 \%$ and increasing) (1370). Type 2 diabetes is often complicated by numerous components of the metabolic syndrome (a syndrome of cardiovascular risk factors affecting over 50 million people in the United States alone, and has been shown to increase cardiovascular disease-related mortality 3.5-fold) and indeed, $\sim 65 \%$ of deaths among people with diabetes are due to cardiovascular disease (1370). The most common macrovascular complication of diabetes is atherosclerosis, which increases the risk for myocardial infarction, stroke, and peripheral artery disease, the latter being the leading cause of limb amputation in civilized countries. Microvascular complications consist of retinopathy and nephropathy, the leading causes of blindness and renal failure (reviewed in Ref. 1177). Diabetes can also lead to the development of diabetic cardiomyopathy, which is characterized by complex changes in the mechanical, structural, biochemical, and electrical properties of the heart, which may underlie the development of an early diastolic and a late systolic dysfunction, or both, and increased incidence of cardiac arrhythmias in diabetic patients (527). Recently, diabetes is considered a potent, independent risk factor for mortality in patients hospitalized with heart failure, particularly in females $(1177,1370)$. 
There is accumulating evidence supporting the key role of NO, superoxide, peroxynitrite, and downstream effectors such as PARP in the pathogenesis of diabetes and diabetic complication (reviewed in Refs. 630,821,958,994,996,997,1204,1232; see also below and Table 5, Figs. 16$18)$.

1. Primary diabetes-Type 1 diabetes (or insulin-dependent diabetes mellitus) is characterized by autoimmune destruction of the pancreatic islet insulin producing $\beta$-cells resulting in prolonged periods of hyperglycemia via reduced uptake of glucose and relative increase in glucagon secretion and gluconeogenesis. Although the actual trigger of the betacell destruction is still unknown, various external (chemicals, viruses) or internal factors (free radicals, cytokines) have been proposed to initiate a deleterious chain of events leading to immune cell infiltration, release of immune mediators such as free radicals and cytokines, and eventually the destruction of $\beta$-cells.

It appears that $\mathrm{NO}$ and various related free radicals and oxidant species are major effectors of $\beta$-cell death $(234,685,1189,1215)$. Consistently, iNOS is overexpressed in the pancreas of diabetic NOD mice (1051), iNOS knockout mice are resistant against streptozotocin-induced diabetes (399), and NOS inhibitors are protective in streptozotocin-induced, NOD, and BB models of type I diabetes $(233,1210,1388)$. The sources of high concentrations of $\mathrm{NO}$ can be infiltrating macrophages and also various cell types of the islet overexpressing iNOS as a result of exposure to inflammatory cytokines such as IL- $1 \beta$, TNF- $\alpha$, and IFN- $\gamma$. Exposure of isolated rat, mouse, and human islets of Langerhans (in vitro models of type 1 diabetes) to various combinations of proinflammatory cytokines leads to inhibition of insulin secretion $(233,345$, $685)$ via NOS induction and subsequent free radical formation (233,685), DNA damage $(307,308,378)$, and consequent PARP activation and depletion of islet ATP and NAD sources, eventually culminating in apoptosis or necrosis $(109,378,474,651,715,1063,1302$; reviewed in Ref. 1232). Furthermore, similar effects could be evoked by exposure of rat and human islets to NO, ROS, or peroxynitrite in vitro $(253,307,308,344,378,504)$.

The first evidence on the possible involvement of endogenously formed peroxynitrite in the $\beta$-cell destruction came from a study demonstrating more frequent nitrotyrosine staining in islets from diabetic NOD mice (22\%) than in islets from normoglycemic NOD mice (7\%) and control BALB/c mice (2\%) (see also Fig. 16). The nitrotyrosine positive cells identified in islets were either macrophages or $\beta$-cells. Most of the $\beta$-cells in islets from diabetic NOD mice were nitrotyrosine positive, whereas only very few were from control animals (1210). Importantly, inhibitors of iNOS and/or scavengers or peroxynitrite prevented development of diabetes and reduced the nitrotyrosine staining in the islets in various experimental models $(822,1209,1210,1234)$.

2. Diabetic cardiovascular dysfunction-Diabetes, and even prediabetes, is characterized by endothelial dysfunction, which precedes and predicts, as well as predisposes, for consequent, more severe vascular alteration $(165,169,1242)$. Considerable evidence suggests that increased oxidative and nitrosative stress in diabetes may play an important role in the development or progression of cardiovascular disease by a number of different mechanisms, including disruption of endothelial function and facilitation of myocardial injury, elevation of acute phase reactants, and impairment of cardiovascular sympathetic nervous system tone and integrity (reviewed in Refs. 630,994,996,997,1204). Hyperglycemia triggers oxidative stress via multiple mechanisms including activation of the polyol pathway, glucose auto-oxidation, alterations of cellular redox state, increased formation of diacylglycerol and the subsequent activation of PKC, and accelerated nonenzymatic formation of advanced glycation end products (AGE) $(150,184,500,946)$. 
Superoxide anion appears to play a particularly important role in the pathogenesis of diabetic cardiovascular dysfunction, and this reactive oxidant was reported to activate many of the above-mentioned pathways (150,184,946;Fig. 17). The cellular sources of superoxide anion are multiple and include $\mathrm{NAD}(\mathrm{P}) \mathrm{H}$ and xanthine oxidases, the arachidonic acid cascade (including cyclooxygenase and lipoxygenase), microsomal enzymes, and the mitochondrial respiratory chain, with the latter being the most important source in diabetes (150;Fig. 17). Hyperglycemia-induced superoxide production leads to increased expression of $\mathrm{NAD}(\mathrm{P}) \mathrm{H}$ oxidases, which in turn generate more superoxide anion. Through the activation of $\mathrm{NF} \kappa \mathrm{B}$, hyperglycemia favors increased expression of iNOS which can increase the generation of NO $(237,1194)$.

Multiple lines of evidence support the pathogenetic role of endogenous peroxynitrite formation in diabetic cardiovascular complications both in experimental animals and in humans (Table 5; Refs. 184,994,996,1204;Figs. 16 and 18). First, increased nitrotyrosine levels are detectable in plasma and platelets of diabetic patients $(36,186,188,190,553,555,850,1265)$, and nitrotyrosine is directly harmful to endothelial cells (879). Second, high glucose causes inactivation of human prostacyclin synthase, increased expression of adhesion molecules, and apoptosis via peroxynitrite formation in cultured human aortic endothelial cells (1473). In cardiomyocytes grown in culture medium containing elevated glucose, pathophysiological alterations can be attenuated by antioxidants, NOS inhibitors, and peroxynitrite scavengers (364). Third, hyperglycemia induces increased nitrotyrosine formation in bovine and human endothelial cells $(236,1040,1049,1473)$, in cultured cardiomyocytes (364), in the artery wall of monkeys (1021), and in diabetic patients during a period of postprandial hyperglycemia (185-189). Fourth, the degree of cell death and/or dysfunction correlates with levels of nitrotyrosine in endothelial cells, cardiomyocytes, and fibroblasts from myocardial biopsies of diabetic patients $(413)$, hearts of experimental diabetic rats $(413,646)$, and hearts perfused with high glucose concentrations (189). Fifth, the nitrotyrosine immunoreactivity is increased in the microvasculature of type 2 diabetic patients and correlates with fasting blood glucose, $\mathrm{HbA1c}$, intracellular adhesion molecule, vascular cellular adhesion molecule, and endothelial dysfunction (1242). Sixth, neutralization of peroxynitrite with peroxynitrite decomposition catalyst ameliorates the endothelial and cardiac dysfunction in streptozotocin-induced murine models of diabetes (1234).

Peroxynitrite has been reported to attack various biomolecules, leading to compromised cardiovascular function in diabetes via numerous mechanisms (Fig. 17). One of these pathways involves DNA strand breakage and consequent activation of the nuclear enzyme PARP, which has been covered by separate overviews $(995,997,1333)$. Figure 18 shows increased nitrotyrosine formation, DNA breaks, and PARP activation in aorta of a diabetic mouse. The PARP-1 activation has recently emerged as a crucial process in the development of diabetic cardiovascular dysfunction both in diabetic animals and humans $(332,989,1185,1242)$ and may also contribute to the development of other diabetic complications such as neuropathy, nephropathy, and retinopathy (for recent reviews, see Refs. 959,997). Recently, a novel mechanism by which PARP activation regulates the development of various pathways related to diabetic complications has been identified $(332,624)$. According to this concept, highglucose-induced elevated superoxide generation from the mitochondria (directly or indirectly via generation of peroxynitrite) induces DNA strand breaks and PARP activation which, in turn, induces the poly(ADP-ribosyl)ation of GAPDH. The resulting metabolic alterations activate $\mathrm{NF} \kappa \mathrm{B}$, polyol pathway, and aldose reductase leading to increased oxidative stress (332,624; Fig. 17).

3. Diabetic neuropathy, nephropathy, and retinopathy-Diabetic nephropathy, neuropathy, and retinopathy develop in at least 30-50\% of patients with chronic diabetes mellitus (irrespective of the type of the diabetes) and are the major cause of increased morbidity 
and mortality. The ultimate consequences of diabetic complications may include renal failure, blindness, and foot ulceration and amputation. Numerous studies have suggested that increased oxidative stress, peroxynitrite formation, and downsream effector pathways such as PARP are involved in the pathogenesis of diabetic microvascular injury in nephropathy, retinopathy, and neuropathy, and this topic is covered by numerous recent overviews $(150,280,630,957,958$, 994,996,1204).

Increased peroxynitrite formation and oxidative stress were demonstrated in kidney biopsies of patients with diabetic nephropathy and in kidneys of diabetic animals $(402,615,974,1277)$. Increased eNOS expression and activity as well as increased formation of superoxide anion and peroxynitrite were found in retinal endothelial cells maintained in high glucose (352, $354)$ and in retinas of diabetic animals $(230,231,354,712,713)$, and all these alterations could be attenuated with various antioxidants, NOS inhibitors, or peroxynitrite scavengers (230, 231,352,354,712). Furthermore, increased peroxynitrite-mediated VEGF and urokinase plasminogen activator receptor expression were proposed to be responsible for the breakdown of the blood-retina barrier in diabetic animals $(352,354)$. Increased formation of peroxynitrite has recently been documented in both experimental $(209,230,231)$ and clinical diabetic neuropathy (554). Furthermore, a peroxynitrite scavenger FP-15 normalized diabetesassociated decreased sciatic motor nerve and digital sensory nerve conduction velocity and ameliorated the nitrotyrosine formation and poly(ADP-ribose) accumulation in diabetic nerves (961). Consistently, several recent studies using PARP-1 knockout mice and/or various PARP inhibitors suggested that PARP activation plays a key role in the pathogenesis of diabetic cardiovascular dysfunction $(332,989,1185,1186,1242)$, nephropathy $(886)$, neuropathy (598, $753,958,960,963)$, and retinopathy (962). Furthermore, some promising antioxidant approaches reduce not only oxidative stress, but also nitrotyrosine formation and PARP activation in diabetic nerves, kidneys and retinas $(331,963)$.

Collectively, the evidence reviewed above support the view that peroxyntrite-induced damage plays an important role in numerous interconnected aspects of the pathogenesis of diabetes and diabetic complications. Neutralization of RNS or inhibition of downstream effector pathways including PARP activation may represent a promising strategy for the prevention or reversal of diabetic complications.

\section{CONCLUSIONS AND FUTURE PERSPECTIVES}

The field of NO has grown immensely from the incredulous proposal as a biological molecule to a field with 80,000 publications and touching all aspects of physiology and pathology. Inhaled NO itself is now routinely administered as a selective pulmonary vasodilator (53, $411,412,593,680,681,1143)$. Drugs affecting the NO pathway have grown into a multibillion dollar business, principally as treatments for male impotence $(159,1066)$. Yet these indications represent only a small fraction of the potential for both improving medical treatment as well as advancing our understanding of the underlying pathophysiology of many diseases.

Understanding the physiology and pathology of NO challenges the paradigms of biological thinking as well as pushes the lower sensitivity limits of analytical chemistry. NO signaling is deceptively simple. In nearly unmeasurable quantities, NO stimulates soluble guanylate cyclase to produce cGMP, which in turn affects intracellular calcium levels to modulate many cellular activities. What is often lost in this simplistic rendition of NO signaling is how the extremely diffusive nature of NO compared with other signaling molecules and moderate halflife allow it to coordinate and integrate physiological responses within small clusters of cells in tissues. A crucial aspect of NO signaling is to act as a shock absorber to dampen physiological responses to prevent parasitic oscillations from overwhelming complex control systems. Its 
diffusive nature also allows NO to act as a retrograde neuromessenger that can transiently affect thousands of synapses within specific areas of the brain.

Overwhelming evidence has established a role for $\mathrm{NO}$ in essentially all major pathological processes affecting humans. But NO itself is unlikely to be the proximal toxin in these processes. It is not highly reactive and is efficiently removed by the reaction with hemoglobin in red blood cells. It takes two oxygen molecules supplied by oxyhemoglobin in red blood cells for NOS to produce each molecule of NO. Because NO diffuses slightly faster than oxygen, it will be able to diffuse back to a red blood cell. Consequently, there will always be an efficient sink to remove NO in blood-perfused tissues that will prevent NO from forming significant amounts of reactive nitrogen species.

However, $\mathrm{NO}$ can be quickly converted into a series of powerful oxidants with many biological effects by its diffusion-limited reactions with many free radicals. The major physiological gateway to produce reactive nitrogen species is most likely through the diffusion-limited reaction with superoxide. While a small flux of superoxide is inevitably produced by leakage of electrons to molecular oxygen through the autoxidation of biological molecules, there is growing recognition that cells can be activated to produce large amounts of superoxide by specific NADPH oxidases and other enzymatic sources. Because the rate of peroxynitrite formation rises 100 -fold for each 10 -fold increase in superoxide and NO production, the production of superoxide offers a dynamic mechanism to redirect NO from being a signaling molecule to an important component of host-defense and innate immunity.

The range of free radical reactions potentially involving $\mathrm{NO}$ is bewildering, leading most investigators to retreat into the relative safety of attributing the effects to terms such as "reactive nitrogen species" or more broadly "reactive nitrogen and oxygen species." But, with this comes a substantial cost in understanding the underlying mechanisms. Once significant amounts of peroxynitrite are produced in a cell, it will produce a shower of other reactive nitrogen species. Peroxynitrite is likely to be the major source of both nitrogen dioxide and possibly nitrite in vivo. When NO is produced more rapidly than superoxide, the excess NO will be consumed to produce a variety of nitroso species, which implies that a modest stimulation of superoxide formation will yield nitrosative rather than nitrative stress.

The broad actions that can be attributed to peroxynitrite in inflammation, cardiovascular disease, neurodegeneration, diabetes, and other pathologies have been reviewed here in some detail. Multiple lines of evidence support the pathophysiological role of peroxynitrite. Its footprints are detectable by using various methods in virtually all diseases both in humans and animals. Potent enzymatic scavenging systems to detoxify peroxynitrite are found in pathogenic microorganisms $(155,156)$. Selective scavengers of peroxynitrite exert beneficial effects in various animal models of disease, and improvements of many pathophysiological conditions in general are associated with decreased target tissue nitrotyrosine formation. Compounds such as urate and many polyphenolics are protective by acting as alternative targets for tyrosine nitration. Many other therapeutic approaches, such as iNOS inhibitors, superoxide scavengers, NADPH oxidase inhibitors, and broadly effective antioxidants (e.g., vitamin E, ascorbate, melatonin, etc.), may be in part protective by preventing the formation of peroxynitrite or repairing damage initiated by it. Various commonly used medications currently used to treat human disease (e.g., ACE inhibitors, carvediol, etc.) also decrease nitrotyrosine formation in diseased tissues. Peroxynitrite may play an important role in modulating vascular injury as well as proinflammatory responses.

The effects of peroxynitrite are not simply the accumulation of random damage to cells as specific responses determine whether cells activate repair processes or die through apoptosis or necrosis. There is a biological specificity to the effects of oxidants that has been largely 
ignored. Hence, enormous opportunities remain to reduce human suffering when these subtle actions are better understood.

\section{Acknowledgements}

P. Pacher wants to acknowledge the support and encouragement of the late Bolfert Iren in the completion of this paper.

GRANTS

This work was supported in part by the Intramural Research Program of the National Institutes of Health (NIH)/NIAAA (to P. Pacher); NIH Grants ES-00040, ES-00240, and AT-02034 (to J. S. Beckman); and Swiss National Fund for Scientific Research Grant PP00B-68882/1 (to L. Liaudet).

\section{References}

1. Abdelkarim GE, Gertz K, Harms C, Katchanov J, Dirnagl U, Szabo C, Endres M. Protective effects of PJ34, a novel, potent inhibitor of poly(ADP-ribose) polymerase (PARP) in in vitro and in vivo models of stroke. Int J Mol Med 2001;7:255-260. [PubMed: 11179503]

2. Abe K, Aoki M, Kawagoe J, Yoshida T, Hattori A, Kogure K, Itoyama Y. Ischemic delayed neuronal death. A mitochondrial hypothesis. Stroke 1995;26:1478-1489. [PubMed: 7631357]

3. Abe K, Pan LH, Watanabe M, Kato T, Itoyama Y. Induction of nitrotyrosine-like immunoreactivity in the lower motor neuron of amyotrophic lateral sclerosis. Neurosci Lett 1995;199:152-154. [PubMed: 8584246]

4. Abe K, Pan LH, Watanabe M, Konno H, Kato T, Itoyama Y. Upregulation of protein-tyrosine nitration in the anterior horn cells of amyotrophic lateral sclerosis. Neurol Res 1997;19:124-128. [PubMed: 9175139]

5. Abou-Mohamed G, Johnson JA, Jin L, El-Remessy AB, Do K, Kaesemeyer WH, Caldwell RB, Caldwell RW. Roles of superoxide, peroxynitrite, and protein kinase $\mathrm{C}$ in the development of tolerance to nitroglycerin. J Pharmacol Exp Ther 2004;308:289-299. [PubMed: 14563789]

6. Acarin L, Peluffo H, Barbeito L, Castellano B, Gonzalez B. Astroglial nitration after postnatal excitotoxic damage: correlation with NO sources, cytoskeletal, apoptotic and antioxidant proteins. J Neurotrauma 2005;22:189-200. [PubMed: 15665612]

7. Adler A, Messina E, Sherman B, Wang Z, Huang H, Linke A, Hintze TH. NAD(P)H oxidase-generated superoxide anion accounts for reduced control of myocardial $\mathrm{O}_{2}$ consumption by NO in old Fischer 344 rats. Am J Physiol Heart Circ Physiol 2003;285:H1015-H1022. [PubMed: 12915388]

8. Ago T, Kitazono T, Kuroda J, Kumai Y, Kamouchi M, Ooboshi H, Wakisaka M, Kawahara T, Rokutan K, Ibayashi S, Iida M. NAD(P)H oxidases in rat basilar arterial endothelial cells. Stroke 2005;36:10401046. [PubMed: 15845888]

9. Ahn B, Ohshima H. Suppression of intestinal polyposis in Apc-(Min/+) mice by inhibiting NO production. Cancer Res 2001;61:8357-8360. [PubMed: 11731407]

10. Akhand AA, Pu M, Senga T, Kato M, Suzuki H, Miyata T, Hamaguchi M, Nakashima I. NO controls src kinase activity through a sulfhydryl group modification-mediated Tyr-527-independent and Tyr-416-linked mechanism. J Biol Chem 1999;274:25821-25826. [PubMed: 10464322]

11. Akizuki E, Akaike T, Okamoto S, Fujii S, Yamaguchi Y, Ogawa M, Maeda H. Role of NO and superoxide in acute cardiac allograft rejection in rats. Proc Soc Exp Biol Med 2000;225:151-159. [PubMed: 11044258]

12. Albertini M, Lafortuna CL, Ciminaghi B, Mazzola S, Clement MG. Endothelin involvement in respiratory centre activity. Prostaglandins Leukot Essent Fatty Acids 2001;65:157-163. [PubMed: 11728166]

13. Allen DA, Harwood S, Varagunam M, Raftery MJ, Yaqoob MM. High glucose-induced oxidative stress causes apoptosis in proximal tubular epithelial cells and is mediated by multiple caspases. FASEB J 2003;17:908-910. [PubMed: 12670885]

14. Altug S, Demiryurek AT, Kane KA, Kanzik I. Evidence for the involvement of peroxynitrite in ischaemic preconditioning in rat isolated hearts. Br J Pharmacol 2000;130:125-131. [PubMed: 10781007] 
15. Alvarez B, Demicheli V, Duran R, Trujillo M, Cervenansky C, Freeman BA, Radi R. Inactivation of human $\mathrm{Cu}, \mathrm{Zn}$ SOD by peroxynitrite and formation of histidinyl radical. Free Radic Biol Med 2004;37:813-822. [PubMed: 15304256]

16. Alvarez B, Radi R. Peroxynitrite reactivity with amino acids and proteins. Amino Acids 2003;25:295311. [PubMed: 14661092]

17. Alvarez S, Boveris A. Mitochondrial NO metabolism in rat muscle during endotoxemia. Free Radic Biol Med 2004;37:1472-1478. [PubMed: 15454287]

18. Ambs S, Merriam WG, Bennett WP, Felley-Bosco E, Ogunfusika MO, Oser SM, Klein S, Shields PG, Billiar TR, Harris CC. Frequent NO synthase-2 expression in human colon adenomas: implication for tumor angiogenesis and colon cancer progression. Cancer Res 1998;58:334-341. [PubMed: 9443414]

19. Ambs S, Merriam WG, Ogunfusika MO, Bennett WP, Ishibe N, Hussain SP, Tzeng EE, Geller DA, Billiar TR, Harris CC. p53 and vascular endothelial growth factor regulate tumor growth of NOS2expressing human carcinoma cells. Nat Med 1998;4:1371-1376. [PubMed: 9846573]

20. Andersen JK. Oxidative stress in neurodegeneration: cause or consequence? Nat Med 2004;10 (Suppl):S18-S25. [PubMed: 15298006]

21. Anderson KE. Huntington's disease and related disorders. Psychiatr Clin North Am 2005;28:275290. [PubMed: 15733623]

22. Andrekopoulos C, Zhang H, Joseph J, Kalivendi S, Kalyanaraman B. Bicarbonate enhances alphasynuclein oligomerization and nitration: intermediacy of carbonate radical anion and nitrogen dioxide radical. Biochem J 2004;378:435-447. [PubMed: 14640973]

23. Andreone TL, O'Connor M, Denenberg A, Hake PW, Zingarelli B. Poly(ADP-ribose) polymerase-1 regulates activation of activator protein-1 in murine fibroblasts. J Immunol 2003;170:2113-2120. [PubMed: 12574383]

24. Anversa P, Nadal-Ginard B. Myocyte renewal and ventricular remodelling. Nature 2002;415:240243. [PubMed: 11805849]

25. Anversa P, Puntillo E, Nikitin P, Olivetti G, Capasso JM, Sonnenblick EH. Effects of age on mechanical and structural properties of myocardium of Fischer 344 rats. Am J Physiol Heart Circ Physiol 1989;256:H1440-H1449.

26. Anversa P, Puntillo E, Olivetti G, Sonnenblick EH, Capasso JM. Load dependence of myocardial growth during maturation and aging. G Ital Cardiol 1989;19:330-342. [PubMed: 2526772]

27. Aoyama K, Matsubara K, Fujikawa Y, Nagahiro Y, Shimizu K, Umegae N, Hayase N, Shiono H, Kobayashi S. Nitration of manganese superoxide dismutase in cerebrospinal fluids is a marker for peroxynitrite-mediated oxidative stress in neurodegenerative diseases. Ann Neurol 2000;47:524527. [PubMed: 10762167]

28. Ara J, Przedborski S, Naini AB, Jackson-Lewis V, Trifiletti RR, Horwitz J, Ischiropoulos H. Inactivation of tyrosine hydroxylase by nitration following exposure to peroxynitrite and 1-methyl-4phenyl-1,2,3,6-tetrahydropyridine (MPTP). Proc Natl Acad Sci USA 1998;95:7659-7663. [PubMed: 9636206]

29. Armstrong JS. Mitochondrial membrane permeabilization: the sine qua non for cell death. Bioessays 2006;28:253-260. [PubMed: 16479581]

30. Arstall MA, Sawyer DB, Fukazawa R, Kelly RA. Cytokine-mediated apoptosis in cardiac myocytes: the role of inducible NO synthase induction and peroxynitrite generation. Circ Res 1999;85:829840. [PubMed: 10532951]

31. Arteel GE, Briviba K, Sies H. Protection against peroxynitrite. FEBS Lett 1999;445:226-230. [PubMed: 10094462]

32. Artz JD, Schmidt B, McCracken JL, Marletta MA. Effects of nitroglycerin on soluble guanylate cyclase: implications for nitrate tolerance. J Biol Chem 2002;277:18253-18256. [PubMed: 11937497]

33. Aslan M, Ozben T. Reactive oxygen and nitrogen species in Alzheimer's disease. Curr Alzheimer Res 2004;1:111-119. [PubMed: 15975075]

34. Augusto O, Bonini MG, Amanso AM, Linares E, Santos CC, De Menezes SL. Nitrogen dioxide and carbonate radical anion: two emerging radicals in biology. Free Radic Biol Med 2002;32:841-859. [PubMed: 11978486] 
35. Aulak KS, Miyagi M, Yan L, West KA, Massillon D, Crabb JW, Stuehr DJ. Proteomic method identifies proteins nitrated in vivo during inflammatory challenge. Proc Natl Acad Sci USA 2001;98:12056-12061. [PubMed: 11593016]

36. Aydin A, Orhan H, Sayal A, Ozata M, Sahin G, Isimer A. Oxidative stress and nitric oxide related parameters in type II diabetes mellitus: effects of glycemic control. Clin Biochem 2001;34:65-70. [PubMed: 11239518]

37. Azevedo LC, Pedro MA, Souza LC, de Souza HP, Janiszewski M, da Luz PL, Laurindo FR. Oxidative stress as a signaling mechanism of the vascular response to injury: the redox hypothesis of restenosis. Cardiovasc Res 2000;47:436-445. [PubMed: 10963717]

38. Bachmaier K, Neu N, Yeung RS, Mak TW, Liu P, Penninger JM. Generation of humanized mice susceptible to peptide-induced inflammatory heart disease. Circulation 1999;99:1885-1891. [PubMed: 10199887]

39. Bachschmid M, Thurau S, Zou MH, Ullrich V. Endothelial cell activation by endotoxin involves superoxide/NO-mediated nitration of prostacyclin synthase and thromboxane receptor stimulation. FASEB J 2003;17:914-916. [PubMed: 12670882]

40. Bachschmid M, van der Loo B, Schuler K, Labugger R, Thurau S, Eto M, Kilo J, Holz R, Luscher $\mathrm{TF}$, Ullrich V. Oxidative stress-associated vascular aging is independent of the protein kinase C/NAD (P)H oxidase pathway. Arch Gerontol Geriatr 2004;38:181-190. [PubMed: 14698497]

41. Bagasra O, Michaels FH, Zheng YM, Bobroski LE, Spitsin SV, Fu ZF, Tawadros R, Koprowski H. Activation of the inducible form of nitric oxide synthase in the brains of patients with multiple sclerosis. Proc Natl Acad Sci USA 1995;92:12041-12045. [PubMed: 8618840]

42. Bagi Z, Ungvari Z, Koller A. Xanthine oxidase-derived reactive oxygen species convert flow-induced arteriolar dilation to constriction in hyperhomocysteinemia: possible role of peroxynitrite. Arterioscler Thromb Vasc Biol 2002;22:28-33. [PubMed: 11788457]

43. Bagnasco P, MacMillan-Crow LA, Greendorfer JS, Young CJ, Andrews L, Thompson JA. Peroxynitrite modulates acidic fibroblast growth factor (FGF-1) activity. Arch Biochem Biophys 2003;419:178-189. [PubMed: 14592461]

44. Bai P, Mabley JG, Liaudet L, Virag L, Szabo C, Pacher P. Matrix metalloproteinase activation is an early event in doxorubicin-induced cardiotoxicity. Oncol Rep 2004;11:505-508. [PubMed: 14719091]

45. Baines CP, Molkentin JD. STRESS signaling pathways that modulate cardiac myocyte apoptosis. J Mol Cell Cardiol 2005;38:47-62. [PubMed: 15623421]

46. Bajt ML, Knight TR, Farhood A, Jaeschke H. Scavenging peroxynitrite with glutathione promotes regeneration and enhances survival during acetaminophen-induced liver injury in mice. J Pharmacol Exp Ther 2003;307:67-73. [PubMed: 12954812]

47. Baker CS, Dutka DP, Pagano D, Rimoldi O, Pitt M, Hall RJ, Polak JM, Bonser RS, Camici PG. Immunocytochemical evidence for inducible nitric oxide synthase and cyclooxygenase-2 expression with nitrotyrosine formation in human hibernating myocardium. Basic Res Cardiol 2002;97:409_ 415. [PubMed: 12200641]

48. Baker CS, Frost MT, Rimoldi O, Moore K, Halliwell B, Polak JM, Camici PG, Hall RJ. Repetitive myocardial stunning in pigs is associated with an increased formation of reactive nitrogen species. Heart 2002;87:77-78. [PubMed: 11751673]

49. Baker CS, Hall RJ, Evans TJ, Pomerance A, Maclouf J, Creminon C, Yacoub MH, Polak JM. Cyclooxygenase- 2 is widely expressed in atherosclerotic lesions affecting native and transplanted human coronary arteries and colocalizes with inducible nitric oxide synthase and nitrotyrosine particularly in macrophages. Arterioscler Thromb Vasc Biol 1999;19:646-655. [PubMed: 10073969]

50. Baker PR, Schopfer FJ, Sweeney S, Freeman BA. Red cell membrane and plasma linoleic acid nitration products: synthesis, clinical identification, and quantitation. Proc Natl Acad Sci USA 2004;101:11577-11582. [PubMed: 15273286]

51. Balaban RS, Nemoto S, Finkel T. Mitochondria, oxidants, and aging. Cell 2005;120:483-495. [PubMed: 15734681]

52. Balafanova Z, Bolli R, Zhang J, Zheng Y, Pass JM, Bhatnagar A, Tang XL, Wang O, Cardwell E, Ping P. Nitric oxide (NO) induces nitration of protein kinase Cepsilon (PKCepsilon), facilitating 
PKCepsilon translocation via enhanced PKCepsilon-RACK2 interactions: a novel mechanism of NO-triggered activation of PKCepsilon. J Biol Chem 2002;277:15021-15027. [PubMed: 11839754]

53. Ballard RA, Truog WE, Cnaan A, Martin RJ, Ballard PL, Merrill JD, Walsh MC, Durand DJ, Mayock DE, Eichenwald EC, Null DR, Hudak ML, Puri AR, Golombek SG, Courtney SE, Stewart DL, Welty SE, Phibbs RH, Hibbs AM, Luan X, Wadlinger SR, Asselin JM, Coburn CE. Inhaled nitric oxide in preterm infants undergoing mechanical ventilation. N Engl J Med 2006;355:343-353. [PubMed: 16870913]

54. Banan A, Fields JZ, Decker H, Zhang Y, Keshavarzian A. Nitric oxide and its metabolites mediate ethanol-induced microtubule disruption and intestinal barrier dysfunction. J Pharmacol Exp Ther 2000;294:997-1008. [PubMed: 10945852]

55. Banan A, Fields JZ, Zhang Y, Keshavarzian A. iNOS upregulation mediates oxidant-induced disruption of F-actin and barrier of intestinal monolayers. Am J Physiol Gastrointest Liver Physiol 2001;280:G1234-G1246. [PubMed: 11352817]

56. Banham HAL, Haldane JS, Savage T. The presence post mortem of nitric-oxide-haemoglobin. $\mathrm{Br}$ Med J Aug 1925;1:187-189.

57. Bapat S, Verkleij A, Post JA. Peroxynitrite activates mitogen-activated protein kinase (MAPK) via a MEK-independent pathway: a role for protein kinase C. FEBS Lett 2001;499:21-26. [PubMed: 11418104]

58. Barbeito LH, Pehar M, Cassina P, Vargas MR, Peluffo H, Viera L, Estevez AG, Beckman JS. A role for astrocytes in motor neuron loss in amyotrophic lateral sclerosis. Brain Res 2004;47:263-274.

59. Barber SC, Mead RJ, Shaw PJ. Oxidative stress in ALS: a mechanism of neurodegeneration and a therapeutic target. Biochim Biophys Acta. In press

60. Barker JE, Bolanos JP, Land JM, Clark JB, Heales SJ. Glutathione protects astrocytes from peroxynitrite-mediated mitochondrial damage: implications for neuronal/astrocytic trafficking and neurodegeneration. Dev Neurosci 1996;18:391-396. [PubMed: 8940610]

61. Barnes PJ, Ito K, Adcock IM. Corticosteroid resistance in chronic obstructive pulmonary disease: inactivation of histone deacetylase. Lancet 2004;363:731-733. [PubMed: 15001333]

62. Barnham KJ, Masters CL, Bush AI. Neurodegenerative diseases and oxidative stress. Nat Rev Drug Discov 2004;3:205-214. [PubMed: 15031734]

63. Bar-Shai M, Reznick AZ. Peroxynitrite induces an alternative NF-kappaB activation pathway in L8 rat myoblasts. Antioxid Redox Signal 2006;8:639-652. [PubMed: 16677107]

64. Bartesaghi S, Valez V, Trujillo M, Peluffo G, Romero N, Zhang H, Kalyanaraman B, Radi R. Mechanistic studies of peroxynitrite-mediated tyrosine nitration in membranes using the hydrophobic probe $N$-t-BOC-L-tyrosine tert-butyl ester. Biochemistry 2006;45:6813-6825. [PubMed: 16734418]

65. Barton $\mathrm{CH}$, Ni Z, Vaziri ND. Enhanced nitric oxide inactivation in aortic coarctation-induced hypertension. Kidney Int 2001;60:1083-1087. [PubMed: 11532103]

66. Battaini F. Protein kinase $\mathrm{C}$ isoforms as therapeutic targets in nervous system disease states. Pharmacol Res 2001;44:353-361. [PubMed: 11712865]

67. Bayir H, Kochanek PM, Clark RS. Traumatic brain injury in infants and children: mechanisms of secondary damage and treatment in the intensive care unit. Crit Care Clin 2003;19:529-549. [PubMed: 12848319]

68. Beal MF. Excitotoxicity and nitric oxide in Parkinson's disease pathogenesis. Ann Neurol 1998;44:S110-S114. [PubMed: 9749581]

69. Beal MF, Ferrante RJ, Browne SE, Matthews RT, Kowall NW, Brown RH Jr. Increased 3nitrotyrosine in both sporadic and familial amyotrophic lateral sclerosis. Ann Neurol 1997;42:644654. [PubMed: 9382477]

70. Becker JS, Adler A, Schneeberger A, Huang H, Wang Z, Walsh E, Koller A, Hintze TH. Hyperhomocysteinemia, a cardiac metabolic disease: role of nitric oxide and the p22phox subunit of NADPH oxidase. Circulation 2005;111:2112-2118. [PubMed: 15851618]

71. Beckman JS. Ischaemic injury mediator. Nature 1990;345:27-28. [PubMed: 2158630]

72. Beckman JS. Oxidative damage and tyrosine nitration from peroxynitrite. Chem Res Toxicol 1996;9:836-844. [PubMed: 8828918]

73. Beckman JS. Peroxynitrite versus hydroxyl radical: the role of nitric oxide in superoxide-dependent cerebral injury. Ann NY Acad Sci 1994;738:69-75. [PubMed: 7832457] 
74. Beckman, JS. The physiological and pathological chemistry of nitric oxide. In: Lancaster, JR., editor. Nitric Oxide: Principles and Actions. Orlando, FL: Academic; 1996. p. 1-82.

75. Beckman JS, Beckman TW, Chen J, Marshall PA, Freeman BA. Apparent hydroxyl radical production by peroxynitrite: implications for endothelial injury from nitric oxide and superoxide. Proc Natl Acad Sci USA 1990;87:1620-1624. [PubMed: 2154753]

76. Beckman JS, Carson M, Smith CD, Koppenol WH. ALS, SOD and peroxynitrite. Nature 1993;364:584. [PubMed: 8350919]

77. Beckman JS, Estevez AG, Crow JP, Barbeito L. Superoxide dismutase and the death of motoneurons in ALS. Trends Neurosci 2001;24:S15-S20. [PubMed: 11881740]

78. Beckman JS, Koppenol WH. Nitric oxide, superoxide, and peroxynitrite: the good, the bad, and ugly. Am J Physiol Cell Physiol 1996;271:C1424-C1437.

79. Beckman JS, Tsai JHM. Reactions and diffusion of nitric oxide and peroxynitrite. Biochemist 1994;16:8-10.

80. Beckman JS, Ye YZ, Anderson PG, Chen J, Accavitti MA, Tarpey MM, White CR. Extensive nitration of protein tyrosines in human atherosclerosis detected by immunohistochemistry. Biol Chem HoppeSeyler 1994;375:81-88. [PubMed: 8192861]

81. Bejma J, Ramires P, Ji LL. Free radical generation and oxidative stress with ageing and exercise: differential effects in the myocardium and liver. Acta Physiol Scand 2000;169:343-351. [PubMed: 10951126]

82. Beller CJ, Radovits T, Kosse J, Gero D, Szabo C, Szabo G. Activation of the peroxynitrite-poly (adenosine diphosphate-ribose) polymerase pathway during neointima proliferation: a new target to prevent restenosis after endarterectomy. J Vasc Surg 2006;43:824-830. [PubMed: 16616243]

83. Benkusky NA, Lewis SJ, Kooy NW. Attenuation of vascular relaxation after development of tachyphylaxis to peroxynitrite in vivo. Am J Physiol Heart Circ Physiol 1998;275:H501-H508.

84. Benkusky NA, Lewis SJ, Kooy NW. Peroxynitrite-mediated attenuation of alpha- and betaadrenoceptor agonist-induced vascular responses in vivo. Eur J Pharmacol 1999;364:151-158. [PubMed: 9932718]

85. Benton DJ, Moore P. Kinetics and mechanism of the formation and decay of peroxynitrous acid in perchloric acid solutions. J Chem Soc 1970:3179-3182.

86. Berger NA. Poly(ADP-ribose) in the cellular response to DNA damage. Radiat Res 1985;101:4-15. [PubMed: 3155867]

87. Berges A, Van Nassauw L, Bosmans J, Timmermans JP, Vrints C. Role of nitric oxide and oxidative stress in ischaemic myocardial injury and preconditioning. Acta Cardiol 2003;58:119-132. [PubMed: 12715903]

88. Berkowitz DE, White R, Li D, Minhas KM, Cernetich A, Kim S, Burke S, Shoukas AA, Nyhan D, Champion HC, Hare JM. Arginase reciprocally regulates nitric oxide synthase activity and contributes to endothelial dysfunction in aging blood vessels. Circulation 2003;108:2000-2006. [PubMed: 14517171]

89. Berlett BS, Friguet B, Yim MB, Chock PB, Stadtman ER. Peroxynitrite-mediated nitration of tyrosine residues in Escherichia coli glutamine synthetase mimics adenylylation: relevance to signal transduction. Proc Natl Acad Sci USA 1996;93:1776-1780. [PubMed: 8700834]

90. Berlett BS, Levine RL, Stadtman ER. Carbon dioxide stimulates peroxynitrite-mediated nitration of tyrosine residues and inhibits oxidation of methionine residues of glutamine synthetase: both modifications mimic effects of adenylylation. Proc Natl Acad Sci USA 1998;95:2784-2789. [PubMed: 9501167]

91. Bertram L, Tanzi RE. The genetic epidemiology of neurodegenerative disease. J Clin Invest 2005;115:1449-1457. [PubMed: 15931380]

92. Besson VC, Croci N, Boulu RG, Plotkine M, Marchand-Verrecchia C. Deleterious poly(ADP-ribose) polymerase-1 pathway activation in traumatic brain injury in rat. Brain Res 2003;989:58-66. [PubMed: 14519512]

93. Besson VC, Margaill I, Plotkine M, Marchand-Verrecchia C. Deleterious activation of poly(ADPribose)polymerase-1 in brain after in vivo oxidative stress. Free Radic Res 2003;37:1201-1208. [PubMed: 14703732] 
94. Besson VC, Zsengeller Z, Plotkine M, Szabo C, Marchand-Verrecchia C. Beneficial effects of PJ34 and INO-1001, two novel water-soluble poly(ADP-ribose) polymerase inhibitors, on the consequences of traumatic brain injury in rat. Brain Res 2005;1041:149-156. [PubMed: 15829224]

95. Beswick RA, Zhang H, Marable D, Catravas JD, Hill WD, Webb RC. Long-term antioxidant administration attenuates mineralocorticoid hypertension and renal inflammatory response. Hypertension 2001;37:781-786. [PubMed: 11230373]

96. Bevan AL, Zhang H, Li Y, Archard LC. Nitric oxide and Cox-sackievirus B3 myocarditis: differential expression of inducible nitric oxide synthase in mouse heart after infection with virulent or attenuated virus. J Med Virol 2001;64:175-182. [PubMed: 11360250]

97. Bharath S, Andersen JK. Glutathione depletion in a midbrain-derived immortalized dopaminergic cell line results in limited tyrosine nitration of mitochondrial complex I subunits: implications for Parkinson's disease. Antioxid Redox Signal 2005;7:900-910. [PubMed: 15998245]

98. Bhattacharya M, Babwah AV, Ferguson SS. Small GTP-binding protein-coupled receptors. Biochem Soc Trans 2004;32:1040-1044. [PubMed: 15506958]

99. Bhusate LL, Herbert KE, Scott DL, Perrett D. Increased DNA strand breaks in mononuclear cells from patients with rheumatoid arthritis. Ann Rheum Dis 1992;51:8-12. [PubMed: 1540043]

100. Bianchi C, Wakiyama H, Faro R, Khan T, McCully JD, Levitsky S, Szabo C, Sellke FW. A novel peroxynitrite decomposer catalyst (FP-15) reduces myocardial infarct size in an in vivo peroxynitrite decomposer and acute ischemia-reperfusion in pigs. Ann Thorac Surg 2002;74:12011207. [PubMed: 12400769]

101. Bidmon HJ, Wu J, Buchkremer-Ratzmann I, Mayer B, Witte OW, Zilles K. Transient changes in the presence of nitric oxide synthases and nitrotyrosine immunoreactivity after focal cortical lesions. Neuroscience 1998;82:377-395. [PubMed: 9466449]

102. Birnboim HC, Lemay AM, Lam DK, Goldstein R, Webb JR. Cutting edge: MHC class II-restricted peptides containing the inflammation-associated marker 3-nitrotyrosine evade central tolerance and elicit a robust cell-mediated immune response. J Immunol 2003;171:528-532. [PubMed: 12847213]

103. Blanchard-Fillion B, Souza JM, Friel T, Jiang GC, Vrana K, Sharov V, Barron L, Schoneich C, Quijano C, Alvarez B, Radi R, Przedborski S, Fernando GS, Horwitz J, Ischiropoulos H. Nitration and inactivation of tyrosine hydroxylase by peroxynitrite. J Biol Chem 2001;276:46017-46023. [PubMed: 11590168]

104. Blough NV, Zafiriou OC. Reaction of superoxide with nitric oxide to form peroxonitrite in alkaline aqueous solution. Inorg Chem 1985;24:3502-3504.

105. Blumberg RS, Saubermann LJ, Strober W. Animal models of mucosal inflammation and their relation to human inflammatory bowel disease. Curr Opin Immunol 1999;11:648-656. [PubMed: 10631550]

106. Boccini F, Herold S. Mechanistic studies of the oxidation of oxyhemoglobin by peroxynitrite. Biochemistry 2004;43:16393-16404. [PubMed: 15610034]

107. Boczkowski J, Lisdero CL, Lanone S, Samb A, Carreras MC, Boveris A, Aubier M, Poderoso JJ. Endogenous peroxynitrite mediates mitochondrial dysfunction in rat diaphragm during endotoxemia. FASEB J 1999;13:1637-1646. [PubMed: 10463956]

108. Bohle DS, Hansert B, Paulson SC, Smith BD. Biomemetic synthesis of the putative cytotoxin peroxynitrite, ONOO-, and its characterization as a tetramethylammonium salt. J Am Chem Soc 1994;116:7423-7424.

109. Bolaffi JL, Rodd GG, Wang J, Grodsky GM. Interrelationship of changes in islet nicotine adeninedinucleotide, insulin secretion, and cell viability induced by interleukin-1 beta. Endocrinology 1994;134:537-542. [PubMed: 7507826]

110. Bolanos JP, Almeida A. Roles of nitric oxide in brain hypoxiaischemia. Biochim Biophys Acta 1999;1411:415-436. [PubMed: 10320673]

111. Bolanos JP, Heales SJ, Land JM, Clark JB. Effect of peroxynitrite on the mitochondrial respiratory chain: differential susceptibility of neurones and astrocytes in primary culture. J Neurochem 1995;64:1965-1972. [PubMed: 7722484]

112. Bolli R, Jeroudi MO, Patel BS, Aruoma OI, Halliwell B, Lai EK, McCay PB. Marked reduction of free radical generation and contractile dysfunction by antioxidant therapy begun at the time of 
reperfusion. Evidence that myocardial "stunning" is a manifestation of reperfusion injury. Circ Res 1989;65:607-622. [PubMed: 2548761]

113. Bolli R, Marban E. Molecular and cellular mechanisms of myocardial stunning. Physiol Rev 1999;79:609-634. [PubMed: 10221990]

114. Boluyt MO, Converso K, Hwang HS, Mikkor A, Russell MW. Echocardiographic assessment of age-associated changes in systolic and diastolic function of the female F344 rat heart. J Appl Physiol 2004;96:822-828. [PubMed: 14555689]

115. Bonfoco E, Krainc D, Ankarcrona M, Nicotera P, Lipton SA. Apoptosis and necrosis: two distinct events induced, respectively, by mild and intense insults with $N$-methyl-D-aspartate or nitric oxide/ superoxide in cortical cell cultures. Proc Natl Acad Sci USA 1995;92:7162-7166. [PubMed: 7638161]

116. Bonizzi G, Karin M. The two NF-kappaB activation pathways and their role in innate and adaptive immunity. Trends Immunol 2004;25:280-288. [PubMed: 15145317]

117. Borbely A, Toth A, Edes I, Virag L, Papp JG, Varro A, Paulus WJ, van der Velden J, Stienen GJ, Papp Z. Peroxynitrite-induced alpha-actinin nitration and contractile alterations in isolated human myocardial cells. Cardiovasc Res 2005;67:225-233. [PubMed: 16005301]

118. Borutaite V, Morkuniene R, Brown GC. Release of cytochrome $c$ from heart mitochondria is induced by high $\mathrm{Ca}^{2+}$ and peroxynitrite and is responsible for $\mathrm{Ca}^{2+}$-induced inhibition of substrate oxidation. Biochim Biophys Acta 1999;1453:41-48. [PubMed: 9989244]

119. Bosse HM, Bachmann S. Immunohistochemically detected protein nitration indicates sites of renal nitric oxide release in Gold-blatt hypertension. Hypertension 1997;30:948-952. [PubMed: 9336398]

120. Bossy-Wetzel E, Talantova MV, Lee WD, Scholzke MN, Harrop A, Mathews E, Gotz T, Han J, Ellisman MH, Perkins GA, Lipton SA. Crosstalk between nitric oxide and zinc pathways to neuronal cell death involving mitochondrial dysfunction and p38-activated $\mathrm{K}^{+}$channels. Neuron 2004;41:351-365. [PubMed: 14766175]

121. Bouchier-Hayes L, Lartigue L, Newmeyer DD. Mitochondria: pharmacological manipulation of cell death. J Clin Invest 2005;115:2640-2647. [PubMed: 16200197]

122. Boulares AH, Yakovlev AG, Ivanova V, Stoica BA, Wang G, Iyer S, Smulson M. Role of poly (ADP-ribose) polymerase (PARP) cleavage in apoptosis. Caspase 3-resistant PARP mutant increases rates of apoptosis in transfected cells. J Biol Chem 1999;274:22932-22940. [PubMed: 10438458]

123. Boullerne AI, Petry KG, Meynard M, Geffard M. Indirect evidence for nitric oxide involvement in multiple sclerosis by characterization of circulating antibodies directed against conjugated $S$ nitrosocysteine. J Neuroimmunol 1995;60:117-124. [PubMed: 7543906]

124. Boulos M, Astiz ME, Barua RS, Osman M. Impaired mitochondrial function induced by serum from septic shock patients is attenuated by inhibition of nitric oxide synthase and poly(ADP-ribose) synthase. Crit Care Med 2003;31:353-358. [PubMed: 12576936]

125. Bouloumie A, Bauersachs J, Linz W, Scholkens BA, Wiemer G, Fleming I, Busse R. Endothelial dysfunction coincides with an enhanced nitric oxide synthase expression and superoxide anion production. Hypertension 1997;30:934-941. [PubMed: 9336396]

126. Boulton CL, Southam E, Garthwaite J. Nitric oxide-dependent long-term potentiation is blocked by a specific inhibitor of soluble guanylyl cyclase. Neuroscience 1995;69:699-703. [PubMed: 8596640]

127. Boveris A, Alvarez S, Navarro A. The role of mitochondrial nitric oxide synthase in inflammation and septic shock. Free Radic Biol Med 2002;33:1186-1193. [PubMed: 12398926]

128. Boyle EM Jr, Canty TG Jr, Morgan EN, Yun W, Pohlman TH, Verrier ED. Treating myocardial ischemia-reperfusion injury by targeting endothelial cell transcription. Ann Thorac Surg 1999;68:1949-1953. [PubMed: 10585109]

129. Brahn E, Banquerigo ML, Firestein GS, Boyle DL, Salzman AL, Szabo C. Collagen induced arthritis: reversal by mercaptoethylguanidine, a novel antiinflammatory agent with a combined mechanism of action. J Rheumatol 1998;25:1785-1793. [PubMed: 9733461]

130. Braughler JM, Mittal CK, Murad F. Effects of thiols, sugars, and proteins on nitric oxide activation of guanylate cyclase. J Biol Chem 1979;254:12450-12454. [PubMed: 40996] 
131. Braunwald E, Kloner RA. The stunned myocardium: prolonged, postischemic ventricular dysfunction. Circulation 1982;66:1146-1149. [PubMed: 6754130]

132. Brealey D, Brand M, Hargreaves I, Heales S, Land J, Smolenski R, Davies NA, Cooper CE, Singer M. Association between mitochondrial dysfunction and severity and outcome of septic shock. Lancet 2002;360:219-223. [PubMed: 12133657]

133. Bredt DS, Hwang PM, Snyder SH. Localization of nitric oxide synthase indicating a neural role for nitric oxide. Nature 1990;347:768-770. [PubMed: 1700301]

134. Bredt DS, Snyder SH. Nitric oxide mediates glutamate-linked enhancement of cGMP levels in the cerebellum. Proc Natl Acad Sci USA 1989;86:9030-9033. [PubMed: 2573074]

135. Bredt DS, Synder HS. Isolation of nitric oxide synthetase, a calmodulin-requiring enzyme. Proc Natl Acad Sci USA 1990;87:682-685. [PubMed: 1689048]

136. Brennan ML, Wu W, Fu X, Shen Z, Song W, Frost H, Vadseth C, Narine L, Lenkiewicz E, Borchers MT, Lusis AJ, Lee JJ, Lee NA, Abu-Soud HM, Ischiropoulos H, Hazen SL. A tale of two controversies: defining both the role of peroxidases in nitrotyrosine formation in vivo using eosinophil peroxidase and myeloperoxidase-deficient mice, and the nature of peroxidase-generated reactive nitrogen species. J Biol Chem 2002;277:17415-17427. [PubMed: 11877405]

137. Brito C, Naviliat M, Tiscornia AC, Vuillier F, Gualco G, Dighiero G, Radi R, Cayota AM. Peroxynitrite inhibits T lymphocyte activation and proliferation by promoting impairment of tyrosine phosphorylation and peroxynitrite-driven apoptotic death. J Immunol 1999;162:33563366. [PubMed: 10092790]

138. Brodsky SV, Gealekman O, Chen J, Zhang F, Togashi N, Crabtree M, Gross SS, Nasjletti A, Goligorsky MS. Prevention and reversal of premature endothelial cell senescence and vasculopathy in obesity-induced diabetes by ebselen. Circ Res 2004;94:377-384. [PubMed: 14670841]

139. Broholm H, Andersen B, Wanscher B, Frederiksen JL, Rubin I, Pakkenberg B, Larsson HB, Lauritzen M. Nitric oxide synthase expression and enzymatic activity in multiple sclerosis. Acta Neurol Scand 2004;109:261-269. [PubMed: 15016008]

140. Brookes PS. Mitochondrial nitric oxide synthase. Mitochondrion 2004;3:187-204. [PubMed: 16120354]

141. Brookes PS, Bolanos JP, Heales SJ. The assumption that nitric oxide inhibits mitochondrial ATP synthesis is correct. FEBS Lett 1999;446:261-263. [PubMed: 10100854]

142. Brookes PS, Darley-Usmar VM. Role of calcium and superoxide dismutase in sensitizing mitochondria to peroxynitrite-induced permeability transition. Am J Physiol Heart Circ Physiol 2004;286:H39-H46. [PubMed: 12933349]

143. Brown GC. Nitric oxide and mitochondrial respiration. Biochim Biophys Acta 1999;1411:351-369. [PubMed: 10320668]

144. Brown GC. Regulation of mitochondrial respiration by nitric oxide inhibition of cytochrome $c$ oxidase. Biochim Biophys Acta 2001;1504:46-57. [PubMed: 11239484]

145. Brown GC, Bal-Price A. Inflammatory neurodegeneration mediated by nitric oxide, glutamate, and mitochondria. Mol Neurobiol 2003;27:325-355. [PubMed: 12845153]

146. Brown GC, Borutaite V. Inhibition of mitochondrial respiratory complex I by nitric oxide, peroxynitrite and $S$-nitrosothiols. Biochim Biophys Acta 2004;1658:44-49. [PubMed: 15282173]

147. Brown GC, Borutaite V. Nitric oxide, cytochrome $c$ and mitochondria. Biochem Soc Symp 1999;66:17-25. [PubMed: 10989653]

148. Brown GC, Borutaite V. Nitric oxide, mitochondria, and cell death. IUBMB Life 2001;52:189-195. [PubMed: 11798032]

149. Brown TH, Chapman PF, Kairiss EW, Keenan CL. Long-term synaptic potentiation. Science 1988;242:724-728. [PubMed: 2903551]

150. Brownlee M. Biochemistry and molecular cell biology of diabetic complications. Nature 2001;414:813-820. [PubMed: 11742414]

151. Bruijn LI, Beal MF, Becher MW, Schulz JB, Wong PC, Price DL, Cleveland DW. Elevated free nitrotyrosine levels, but not protein-bound nitrotyrosine or hydroxyl radicals, throughout amyotrophic lateral sclerosis (ALS)-like disease implicate tyrosine nitration as an aberrant in vivo property of one familial ALS-linked superoxide dismutase 1 mutant. Proc Natl Acad Sci USA 1997;94:7606-7611. [PubMed: 9207139] 
152. Bruijn LI, Miller TM, Cleveland DW. Unraveling the mechanisms involved in motor neuron degeneration in ALS. Annu Rev Neurosci 2004;27:723-749. [PubMed: 15217349]

153. Brunelli L, Yermilov V, Beckman JS. Modulation of catalase peroxidatic and catalatic activity by nitric oxide. Free Radic Biol Med 2001;30:709-714. [PubMed: 11275470]

154. Brüning G. NADPH-diaphorase histochemistry in the postnatal mouse cerebellum suggests specific development functions for nitric oxide. J Neurosci Res 1993;36:580-587. [PubMed: 7511699]

155. Bryk R, Griffin P, Nathan C. Peroxynitrite reductase activity of bacterial peroxiredoxins. Nature 2000;407:211-215. [PubMed: 11001062]

156. Bryk R, Lima CD, Erdjument-Bromage H, Tempst P, Nathan C. Metabolic enzymes of mycobacteria linked to antioxidant defense by a thioredoxin-like protein. Science 2002;295:1073-1077. [PubMed: 11799204]

157. Buchczyk DP, Grune T, Sies H, Klotz LO. Modifications of glyceraldehyde-3-phosphate dehydrogenase induced by increasing concentrations of peroxynitrite: early recognition by $20 \mathrm{~S}$ proteasome. Biol Chem 2003;384:237-241. [PubMed: 12675516]

158. Burner U, Furtmüller PG, Kettle AJ, Koppenol WH, Obinger C. Mechanism of reaction of myeloperoxidase with nitrite. J Biol Chem 2000;275:20597-20601. [PubMed: 10777476]

159. Burnett AL, Musicki B, Jin L, Bivalacqua TJ. Nitric oxide/redox-based signalling as a therapeutic target for penile disorders. Expert Opin Ther Targets 2006;10:445-457. [PubMed: 16706684]

160. Burney S, Caulfield JL, Niles JC, Wishnok JS, Tannenbaum SR. The chemistry of DNA damage from nitric oxide and peroxynitrite. Mutat Res 1999;424:37-49. [PubMed: 10064848]

161. Butler AR, Megson IL, Wright PG. Diffusion of nitric oxide and scavenging by blood in the vasculature. Biochim Biophys Acta 1998;1425:168-176. [PubMed: 9813307]

162. Butterfield DA, Boyd-Kimball D. Amyloid beta-peptide(1-42) contributes to the oxidative stress and neurodegeneration found in Alzheimer disease brain. Brain Pathol 2004;14:426-432. [PubMed: 15605990]

163. Butterfield DA, Howard BJ, LaFontaine MA. Brain oxidative stress in animal models of accelerated aging and the age-related neurodegenerative disorders, Alzheimer's disease and Huntington's disease. Curr Med Chem 2001;8:815-828. [PubMed: 11375752]

164. Buttery LD, Springall DR, Chester AH, Evans TJ, Standfield EN, Parums DV, Yacoub MH, Polak JM. Inducible nitric oxide synthase is present within human atherosclerotic lesions and promotes the formation and activity of peroxynitrite. Lab Invest 1996;75:77-85. [PubMed: 8683942]

165. Caballero AE, Arora S, Saouaf R, Lim SC, Smakowski P, Park JY, King GL, LoGerfo FW, Horton ES, Veves A. Microvascular and macrovascular reactivity is reduced in subjects at risk for type 2 diabetes. Diabetes 1999;48:1856-1862. [PubMed: 10480619]

166. Cai H, Harrison DG. Endothelial dysfunction in cardiovascular diseases: the role of oxidant stress. Circ Res 2000;87:840-844. [PubMed: 11073878]

167. Cai H, McNally JS, Weber M, Harrison DG. Oscillatory shear stress upregulation of endothelial nitric oxide synthase requires intracellular hydrogen peroxide and CaMKII. J Mol Cell Cardiol 2004;37:121-125. [PubMed: 15242742]

168. Calabrese V, Scapagnini G, Ravagna A, Bella R, Foresti R, Bates TE, Giuffrida Stella AM, Pennisi G. Nitric oxide synthase is present in the cerebrospinal fluid of patients with active multiple sclerosis and is associated with increases in cerebrospinal fluid protein nitrotyrosine and $S$-nitrosothiols and with changes in glutathione levels. J Neurosci Res 2002;70:580-587. [PubMed: 12404512]

169. Calles-Escandon J, Cipolla M. Diabetes and endothelial dysfunction: a clinical perspective. Endocr Rev 2001;22:36-52. [PubMed: 11159815]

170. Calo L, Giacon B, Davis PA, Pagnin E, Piccin A, Riegler P, Huber W, Antonello A, Semplicini A. Oxidative stress and TGFbeta in kidney-transplanted patients with cyclosporin-induced hypertension. Effect of carvedilol and nifedipine. Clin Nephrol 2002;58:103-110. [PubMed: 12227681]

171. Capasso JM, Palackal T, Olivetti G, Anversa P. Severe myocardial dysfunction induced by ventricular remodeling in aging rat hearts. Am J Physiol Heart Circ Physiol 1990;259:H1086H1096. 
172. Cardillo C, Kilcoyne CM, Quyyumi AA, Cannon RO 3rd, Panza JA. Selective defect in nitric oxide synthesis may explain the impaired endothelium-dependent vasodilation in patients with essential hypertension. Circulation 1998;97:851-856. [PubMed: 9521333]

173. Carri MT, Ferri A, Cozzolino M, Calabrese L, Rotilio G. Neurodegeneration in amyotrophic lateral sclerosis: the role of oxidative stress and altered homeostasis of metals. Brain Res Bull 2003;61:365-374. [PubMed: 12909279]

174. Carter CA, Kane CJ. Therapeutic potential of natural compounds that regulate the activity of protein kinase C. Curr Med Chem 2004;11:2883-2902. [PubMed: 15544481]

175. Caselli A, Camici G, Manao G, Moneti G, Pazzagli L, Cappugi G, Ramponi G. Nitric oxide causes inactivation of the low molecular weight phosphotyrosine protein phosphatase. J Biol Chem 1994;269:24878-24882. [PubMed: 7929168]

176. Casoni F, Basso M, Massignan T, Gianazza E, Cheroni C, Salmona M, Bendotti C, Bonetto V. Protein nitration in a mouse model of familial amyotrophic lateral sclerosis: possible multifunctional role in the pathogenesis. J Biol Chem 2005;280:16295-16304. [PubMed: 15699043]

177. Cassina A, Radi R. Differential inhibitory action of nitric oxide and peroxynitrite on mitochondrial electron transport. Arch Biochem Biophys 1996;328:309-316. [PubMed: 8645009]

178. Cassina AM, Hodara R, Souza JM, Thomson L, Castro L, Ischiropoulos H, Freeman BA, Radi R. Cytochrome $c$ nitration by peroxynitrite. J Biol Chem 2000;275:21409-21415. [PubMed: 10770952]

179. Cassina P, Peluffo H, Pehar M, Martinez-Palma L, Ressia A, Beckman JS, Estevez AG, Barbeito L. Peroxynitrite triggers a phenotypic transformation in spinal cord astrocytes that induces motor neuron apoptosis. J Neurosci Res 2002;67:21-29. [PubMed: 11754077]

180. Castegna A, Thongboonkerd V, Klein JB, Lynn B, Markesbery WR, Butterfield DA. Proteomic identification of nitrated proteins in Alzheimer's disease brain. J Neurochem 2003;85:1394-1401. [PubMed: 12787059]

181. Castillo J, Rama R, Davalos A. Nitric oxide-related brain damage in acute ischemic stroke. Stroke 2000;31:852-857. [PubMed: 10753987]

182. Castro L, Rodriguez M, Radi R. Aconitase is readily inactivated by peroxynitrite, but not by its precursor, nitric oxide. J Biol Chem 1994;269:29409-29415. [PubMed: 7961920]

183. Cayatte AJ, Palacino JJ, Horten K, Cohen RA. Chronic inhibition of nitric oxide production accelerates neointima formation and impairs endothelial function in hypercholesterolemic rabbits. Arterioscler Thromb 1994;14:753-759. [PubMed: 7513551]

184. Ceriello A. New insights on oxidative stress and diabetic complications may lead to a "causal" antioxidant therapy. Diabetes Care 2003;26:1589-1596. [PubMed: 12716823]

185. Ceriello A. Nitrotyrosine: new findings as a marker of postprandial oxidative stress. Int J Clin Pract Suppl 2002:51-58. [PubMed: 12166608]

186. Ceriello A, Mercuri F, Quagliaro L, Assaloni R, Motz E, Tonutti L, Taboga C. Detection of nitrotyrosine in the diabetic plasma: evidence of oxidative stress. Diabetologia 2001;44:834-838. [PubMed: 11508267]

187. Ceriello A, Piconi L, Quagliaro L, Wang Y, Schnabel CA, Ruggles JA, Gloster MA, Maggs DG, Weyer C. Effects of pramlintide on postprandial glucose excursions and measures of oxidative stress in patients with type 1 diabetes. Diabetes Care 2005;28:632-637. [PubMed: 15735200]

188. Ceriello A, Quagliaro L, Catone B, Pascon R, Piazzola M, Bais B, Marra G, Tonutti L, Taboga C, Motz E. Role of hyperglycemia in nitrotyrosine postprandial generation. Diabetes Care 2002;25:1439-1443. [PubMed: 12145247]

189. Ceriello A, Quagliaro L, D’Amico M, Di Filippo C, Marfella R, Nappo F, Berrino L, Rossi F, Giugliano D. Acute hyperglycemia induces nitrotyrosine formation and apoptosis in perfused heart from rat. Diabetes 2002;51:1076-1082. [PubMed: 11916928]

190. Ceriello A, Taboga C, Tonutti L, Quagliaro L, Piconi L, Bais B, Da Ros R, Motz E. Evidence for an independent and cumulative effect of postprandial hypertriglyceridemia and hyperglycemia on endothelial dysfunction and oxidative stress generation: effects of short- and long-term simvastatin treatment. Circulation 2002;106:1211-1218. [PubMed: 12208795] 
191. Cerioni L, Palomba L, Cantoni O. The Raf/MEK inhibitor PD98059 enhances ERK1/2 phosphorylation mediated by peroxynitrite via enforced mitochondrial formation of reactive oxygen species. FEBS Lett 2003;547:92-96. [PubMed: 12860392]

192. Cesselli D, Jakoniuk I, Barlucchi L, Beltrami AP, Hintze TH, Nadal-Ginard B, Kajstura J, Leri A, Anversa P. Oxidative stress-mediated cardiac cell death is a major determinant of ventricular dysfunction and failure in dog dilated cardiomyopathy. Circ Res 2001;89:279-286. [PubMed: 11485979]

193. Chabrier PE, Auguet M, Spinnewyn B, Auvin S, Cornet S, Demerle-Pallardy C, Guilmard-Favre C, Marin JG, Pignol B, Gillard-Roubert V, Roussillot-Charnet C, Schulz J, Viossat I, Bigg D, Moncada S. BN 80933, a dual inhibitor of neuronal nitric oxide synthase and lipid peroxidation: a promising neuroprotective strategy. Proc Natl Acad Sci USA 1999;96:10824-10829. [PubMed: 10485910]

194. Chabrier PE, Demerle-Pallardy C, Auguet M. Nitric oxide synthases: targets for therapeutic strategies in neurological diseases. Cell Mol Life Sci 1999;55:1029-1035. [PubMed: 10442086]

195. Chakraborti T, Das S, Chakraborti S. Proteolytic activation of protein kinase Calpha by peroxynitrite in stimulating cytosolic phospholipase $\mathrm{A}_{2}$ in pulmonary endothelium: involvement of a pertussis toxin sensitive protein. Biochemistry 2005;44:5246-5257. [PubMed: 15794661]

196. Chakraborty S, Balakotaiah V, Bidani A. Diffusing capacity reexamined: relative roles of diffusion and chemical reaction in red cell uptake of. J Appl Physiol 2004;97O $2, \mathrm{CO}, \mathrm{CO}_{2}$, and NO. 22842302

197. Chandrasekar B, Colston JT, Freeman GL. Induction of proinflammatory cytokine and antioxidant enzyme gene expression following brief myocardial ischaemia. Clin Exp Immunol 1997; 108:346351. [PubMed: 9158109]

198. Chang L, Karin M. Mammalian MAP kinase signalling cascades. Nature 2001;410:37-40. [PubMed: 11242034]

199. Chang W, Webster DR, Salam AA, Gruber D, Prasad A, Eiserich JP, Bulinski JC. Alteration of the C-terminal amino acid of tubulin specifically inhibits myogenic differentiation. J Biol Chem 2002;277:30690-30698. [PubMed: 12070174]

200. Chatterjee PK, Patel NS, Sivarajah A, Kvale EO, Dugo L, Cuzzocrea S, Brown PA, Stewart KN, Mota-Filipe H, Britti D, Yaqoob MM, Thiemermann C. GW274150, a potent and highly selective inhibitor of iNOS, reduces experimental renal ischemia/reperfusion injury. Kidney Int 2003;63:853-865. [PubMed: 12631066]

201. Chaves AA, Mihm MJ, Schanbacher BL, Basuray A, Liu C, Ayers LW, Bauer JA. Cardiomyopathy in a murine model of AIDS: evidence of reactive nitrogen species and corroboration in human HIV/ AIDS cardiac tissues. Cardiovasc Res 2003;60:108-118. [PubMed: 14522412]

202. Chavez AM, Menconi MJ, Hodin RA, Fink MP. Cytokine-induced intestinal epithelial hyperpermeability: role of nitric oxide. Crit Care Med 1999;27:2246-2251. [PubMed: 10548215]

203. Chen J, Kuhlencordt P, Urano F, Ichinose H, Astern J, Huang PL. Effects of chronic treatment with L-arginine on atherosclerosis in apoE knockout and apoE/inducible NO synthase double-knockout mice. Arterioscler Thromb Vasc Biol 2003;23:97-103. [PubMed: 12524231]

204. Chen L, Fuller CM, Kleyman TR, Matalon S. Mutations in the extracellular loop of alpha-rENaC alter sensitivity to amiloride and reactive species. Am J Physiol Renal Physiol 2004;286:F1202F1208. [PubMed: 14969999]

205. Chen LW, Egan L, Li ZW, Greten FR, Kagnoff MF, Karin M. The two faces of IKK and NF-kappaB inhibition: prevention of systemic inflammation but increased local injury following intestinal ischemia-reperfusion. Nat Med 2003;9:575-581. [PubMed: 12692538]

206. Chen M, Ona VO, Li M, Ferrante RJ, Fink KB, Zhu S, Bian J, Guo L, Farrell LA, Hersch SM, Hobbs W, Vonsattel JP, Cha JH, Friedlander RM. Minocycline inhibits caspase-1 and caspase-3 expression and delays mortality in a transgenic mouse model of Huntington disease. Nat Med 2000;6:797-801. [PubMed: 10888929]

207. Chen T, Zamora R, Zuckerbraun B, Billiar TR. Role of nitric oxide in liver injury. Curr Mol Med 2003;3:519-526. [PubMed: 14527083]

208. Chen Y, Traverse JH, Du R, Hou M, Bache RJ. Nitric oxide modulates myocardial oxygen consumption in the failing heart. Circulation 2002;106:273-279. [PubMed: 12105170] 
209. Cheng C, Zochodne DW. Sensory neurons with activated caspase-3 survive long-term experimental diabetes. Diabetes 2003;52:2363-2371. [PubMed: 12941777]

210. Cheng XS, Shimokawa H, Momii H, Oyama J, Fukuyama N, Egashira K, Nakazawa H, Takeshita A. Role of superoxide anion in the pathogenesis of cytokine-induced myocardial dysfunction in dogs in vivo. Cardiovasc Res 1999;42:651-659. [PubMed: 10533605]

211. Cheung PY, Danial H, Jong J, Schulz R. Thiols protect the inhibition of myocardial aconitase by peroxynitrite. Arch Biochem Biophys 1998;350:104-108. [PubMed: 9466826]

212. Cheung PY, Wang W, Schulz R. Glutathione protects against myocardial ischemia-reperfusion injury by detoxifying peroxynitrite. J Mol Cell Cardiol 2000;32:1669-1678. [PubMed: 10966829]

213. Chi Q, Wang T, Huang K. Effect of insulin nitration by peroxynitrite on its biological activity. Biochem Biophys Res Commun 2005;330:791-796. [PubMed: 15809066]

214. Chiarugi A. Poly(ADP-ribosyl)ation and stroke. Pharmacol Res 2005;52:15-24. [PubMed: 15911330]

215. Cho HJ, Xie QW, Calaycay J, Mumford RA, Swiderek KM, Lee TD, Nathan C. Calmodulin is a subunit of nitric oxide synthase from macrophages. J Exp Med 1992;176:599-604. [PubMed: 1380065]

216. Choi JW, Yoo BK, Ryu MK, Choi MS, Park GH, Ko KH. Adenosine and purine nucleosides prevent the disruption of mitochondrial transmembrane potential by peroxynitrite in rat primary astrocytes. Arch Pharm Res 2005;28:810-815. [PubMed: 16114496]

217. Chou SM, Wang HS, Komai K. Colocalization of NOS and SOD1 in neurofilament accumulation within motor neurons of amyotrophic lateral sclerosis: an immunohistochemical study. J Chem Neuroanat 1996;10:249-258. [PubMed: 8811414]

218. Chou SM, Wang HS, Taniguchi A. Role of SOD-1 and nitric oxide/cyclic GMP cascade on neurofilament aggregation in ALS/MND. J Neurol Sci 1996;139(Suppl):16-26. [PubMed: 8899653]

219. Chung KK, Dawson TM, Dawson VL. Nitric oxide, $S$-nitrosylation and neurodegeneration. Cell Mol Biol 2005;51:247-254. [PubMed: 16191392]

220. Cleeter MWJ, Cooper JM, Darley-Usmar VM, Moncada S, Schapira AHV. Reversible inhibition of cytochrome $c$ oxidase, the terminal enzyme of the mitochondrial respiratory chain, by nitric oxide. FEBS Lett 1994;345:50-54. [PubMed: 8194600]

221. Clements MK, Siemsen DW, Swain SD, Hanson AJ, Nelson-Overton LK, Rohn TT, Quinn MT. Inhibition of actin polymerization by peroxynitrite modulates neutrophil functional responses. J Leukoc Biol 2003;73:344-355. [PubMed: 12629148]

222. Cobbs CS, Samanta M, Harkins LE, Gillespie GY, Merrick BA, MacMillan-Crow LA. Evidence for peroxynitrite-mediated modifications to p53 in human gliomas: possible functional consequences. Arch Biochem Biophys 2001;394:167-172. [PubMed: 11594730]

223. Cobbs CS, Whisenhunt TR, Wesemann DR, Harkins LE, Van Meir EG, Samanta M. Inactivation of wild-type $\mathrm{p} 53$ protein function by reactive oxygen and nitrogen species in malignant glioma cells. Cancer Res 2003;63:8670-8673. [PubMed: 14695179]

224. Coddington JW, Hurst JK, Lymar SV. Hydroxyl radical formation during peroxynitrous acid decomposition. J Am Chem Soc 1999;121:2438-2443.

225. Coma M, Guix FX, Uribesalgo I, Espuna G, Sole M, Andreu D, Munoz FJ. Lack of oestrogen protection in amyloid-mediated endothelial damage due to protein nitrotyrosination. Brain 2005;128:1613-1621. [PubMed: 15817516]

226. Compston A, Coles A. Multiple sclerosis. Lancet 2002;359:1221-1231. [PubMed: 11955556]

227. Connor JR, Manning PT, Settle SL, Moore WM, Jerome GM, Webber RK, Tjoeng FS, Currie MG. Suppression of adjuvant-induced arthritis by selective inhibition of inducible nitric oxide synthase. Eur J Pharmacol 1995;273:15-24. [PubMed: 7537678]

228. Cooke CL, Davidge ST. Peroxynitrite increases iNOS through NF-kappaB and decreases prostacyclin synthase in endothelial cells. Am J Physiol Cell Physiol 2002;282:C395-C402. [PubMed: 11788351]

229. Cooke JP, Singer AH, Tsao P, Zera P, Rowan RA, Billingham ME. Antiatherogenic effects of Larginine in the hypercholesterolemic rabbit. J Clin Invest 1992;90:1168-1172. [PubMed: 1522225] 
230. Coppey LJ, Gellett JS, Davidson EP, Dunlap JA, Lund DD, Salvemini D, Yorek MA. Effect of M40403 treatment of diabetic rats on endoneurial blood flow, motor nerve conduction velocity and vascular function of epineurial arterioles of the sciatic nerve. Br J Pharmacol 2001;134:21-29. [PubMed: 11522593]

231. Coppey LJ, Gellett JS, Davidson EP, Dunlap JA, Lund DD, Yorek MA. Effect of antioxidant treatment of streptozotocin-induced diabetic rats on endoneurial blood flow, motor nerve conduction velocity, and vascular reactivity of epineurial arterioles of the sciatic nerve. Diabetes 2001;50:1927-1937. [PubMed: 11473057]

232. Coppey LJ, Gellett JS, Davidson EP, Yorek MA. Preventing superoxide formation in epineurial arterioles of the sciatic nerve from diabetic rats restores endothelium-dependent vasodilation. Free Radic Res 2003;37:33-40. [PubMed: 12653215]

233. Corbett JA, Sweetland MA, Wang JL, Lancaster JR Jr, Mc-Daniel ML. Nitric oxide mediates cytokine-induced inhibition of insulin secretion by human islets of Langerhans. Proc Natl Acad Sci USA 1993;90:1731-1735. [PubMed: 8383325]

234. Corbett JA, Wang JL, Sweetland MA, Lancaster JR Jr, Mc-Daniel ML. Interleukin 1 beta induces the formation of nitric oxide by beta-cells purified from rodent islets of Langerhans. Evidence for the beta-cell as a source and site of action of nitric oxide. J Clin Invest 1992;90:2384-2391. [PubMed: 1334975]

235. Corcoran GB, Bauer JA, Lau TW. Immediate rise in intracellular calcium and glycogen phosphorylase a activities upon acetaminophen covalent binding leading to hepatotoxicity in mice. Toxicology 1988;50:157-167. [PubMed: 3388436]

236. Cosentino F, Eto M, De Paolis P, van der Loo B, Bachschmid M, Ullrich V, Kouroedov A, Delli Gatti C, Joch H, Volpe M, Luscher TF. High glucose causes upregulation of cyclooxygenase- 2 and alters prostanoid profile in human endothelial cells: role of protein kinase $\mathrm{C}$ and reactive oxygen species. Circulation 2003;107:1017-1023. [PubMed: 12600916]

237. Cosentino F, Hishikawa K, Katusic ZS, Luscher TF. High glucose increases nitric oxide synthase expression and superoxide anion generation in human aortic endothelial cells. Circulation 1997;96:25-28. [PubMed: 9236411]

238. Coussens LM, Werb Z. Inflammation and cancer. Nature 2002;420:860-867. [PubMed: 12490959]

239. Cover C, Mansouri A, Knight TR, Bajt ML, Lemasters JJ, Pessayre D, Jaeschke H. Peroxynitriteinduced mitochondrial and endonuclease-mediated nuclear DNA damage in acetaminophen hepatotoxicity. J Pharmacol Exp Ther 2005;315:879-887. [PubMed: 16081675]

240. Cromheeke KM, Kockx MM, De Meyer GR, Bosmans JM, Bult H, Beelaerts WJ, Vrints CJ, Herman AG. Inducible nitric oxide synthase colocalizes with signs of lipid oxidation/peroxidation in human atherosclerotic plaques. Cardiovasc Res 1999;43:744-754. [PubMed: 10690346]

241. Cross AH, Manning PT, Keeling RM, Schmidt RE, Misko TP. Peroxynitrite formation within the central nervous system in active multiple sclerosis. J Neuroimmunol 1998;88:45-56. [PubMed: 9688323]

242. Cross AH, Misko TP, Lin RF, Hickey WF, Trotter JL, Tilton RG. Aminoguanidine, an inhibitor of inducible nitric oxide synthase, ameliorates experimental autoimmune encephalomyelitis in SJL mice. J Clin Invest 1994;93:2684-2690. [PubMed: 7515395]

243. Crouser ED, Julian MW, Weinstein DM, Fahy RJ, Bauer JA. Endotoxin-induced ileal mucosal injury and nitric oxide dysregulation are temporally dissociated. Am J Respir Crit Care Med 2000;161:1705-1712. [PubMed: 10806178]

244. Crow JP, Beckman JS. Quantitation of protein tyrosine, 3-nitrotyrosine, and 3-aminotyrosine utilizing HPCL and intrinsic ultraviolet absorbance. Methods 1995;7:116-120.

245. Crow JP, Beckman JS, McCord JM. Sensitivity of the essential zinc-thiolate moiety of yeast alcohol dehydrogenase to hypochlorite and peroxynitrite. Biochemistry 1995;34:3544-3552. [PubMed: 7893650]

246. Crow JP, Sampson JB, Zhuang Y, Thompson JA, Beckman JS. Decreased zinc affinity of amyotrophic lateral sclerosis-associated superoxide dismutase mutants leads to enhanced catalysis of tyrosine nitration by peroxynitrite. J Neurochem 1997;69:1936-1944. [PubMed: 9349538] 
247. Crow JP, Ye YZ, Strong M, Kirk M, Barnes S, Beckman JS. Superoxide dismutase catalyzes nitration of tyrosines by peroxynitrite in the rod and head domains of neurofilament-L. J Neurochem 1997;69:1945-1953. [PubMed: 9349539]

248. Csiszar A, Pacher P, Kaley G, Ungvari Z. Role of oxidative and nitrosative stress, longevity genes and poly(ADP-ribose) polymerase in cardiovascular dysfunction associated with aging. Curr Vasc Pharmacol 2005;3:285-291. [PubMed: 16026324]

249. Csiszar A, Ungvari Z, Edwards JG, Kaminski P, Wolin MS, Koller A, Kaley G. Aging-induced phenotypic changes and oxidative stress impair coronary arteriolar function. Circ Res 2002;90:1159-1166. [PubMed: 12065318]

250. Csiszar A, Ungvari Z, Koller A, Edwards JG, Kaley G. Aging-induced proinflammatory shift in cytokine expression profile in coronary arteries. FASEB J 2003;17:1183-1185. [PubMed: 12709402]

251. Csiszar A, Ungvari Z, Koller A, Edwards JG, Kaley G. Proinflammatory phenotype of coronary arteries promotes endothelial apoptosis in aging. Physiol Gen 2004;17:21-30.

252. Csonka C, Csont T, Onody A, Ferdinandy P. Preconditioning decreases ischemia/reperfusioninduced peroxynitrite formation. Biochem Biophys Res Commun 2001;285:1217-1219. [PubMed: 11478785]

253. Cunningham JM, Mabley JG, Delaney CA, Green IC. The effect of nitric oxide donors on insulin secretion, cyclic GMP and cyclic AMP in rat islets of Langerhans and the insulin-secreting cell lines HIT-T15 and RINm5F. Mol Cell Endocrinol 1994;102:23-29. [PubMed: 7926270]

254. Cuzzocrea S. Shock, inflammation and PARP. Pharmacol Res 2005;52:72-82. [PubMed: 15911335]

255. Cuzzocrea S, Caputi AP. Protective effect of melatonin on zymosan-induced cellular damage. Biol Signals Recept 1999;8:136-142. [PubMed: 10085476]

256. Cuzzocrea S, Chatterjee PK, Mazzon E, McDonald MC, Dugo L, Di Paola R, Serraino I, Britti D, Caputi AP, Thiemermann C. Beneficial effects of GW274150, a novel, potent and selective inhibitor of iNOS activity, in a rodent model of collagen-induced arthritis. Eur J Pharmacol 2002;453:119129. [PubMed: 12393067]

257. Cuzzocrea S, Costantino G, Caputi AP. Protective effect of $N$-acetylcysteine on cellular energy depletion in a non-septic shock model induced by zymosan in the rat. Shock 1999;11:143-148. [PubMed: 10030802]

258. Cuzzocrea S, Costantino G, Mazzon E, Caputi AP. Protective effect of $N$-acetylcysteine on multiple organ failure induced by zymosan in the rat. Crit Care Med 1999;27:1524-1532. [PubMed: 10470760]

259. Cuzzocrea S, Costantino G, Mazzon E, De Sarro A, Caputi AP. Beneficial effects of Mn(III)tetrakis (4-benzoic acid)porphyrin (MnTBAP), a superoxide dismutase mimetic, in zymosan-induced shock. Br J Pharmacol 1999;128:1241-1251. [PubMed: 10578138]

260. Cuzzocrea S, Costantino G, Mazzon E, Micali A, De Sarro A, Caputi AP. Beneficial effects of melatonin in a rat model of splanchnic artery occlusion and reperfusion. J Pineal Res 2000;28:5263. [PubMed: 10626602]

261. Cuzzocrea S, Costantino G, Mazzon E, Zingarelli B, De Sarro A, Caputi AP. Protective effects of $\mathrm{Mn}$ (III)tetrakis(4-benzoic acid)porphyrin (MnTBAP), a superoxide dismutase mimetic, in paw oedema induced by carrageenan in the rat. Biochem Pharmacol 1999;58:171-176. [PubMed: 10403531]

262. Cuzzocrea S, Costantino G, Zingarelli B, Mazzon E, Micali A, Caputi AP. The protective role of endogenous glutathione in carrageenan-induced pleurisy in the rat. Eur J Pharmacol 1999;372:187197. [PubMed: 10395099]

263. Cuzzocrea S, Mazzon E, Costantino G, Serraino I, De Sarro A, Caputi AP. Effects of $n$-acetylcysteine in a rat model of ischemia and reperfusion injury. Cardiovasc Res 2000;47:537-548. [PubMed: 10963726]

264. Cuzzocrea S, Mazzon E, Costantino G, Serraino I, Dugo L, Calabro G, Cucinotta G, De Sarro A, Caputi AP. Beneficial effects of $n$-acetylcysteine on ischaemic brain injury. Br J Pharmacol 2000;130:1219-1226. [PubMed: 10903958] 
265. Cuzzocrea S, Mazzon E, Di Paola R, Esposito E, Macarthur H, Matuschak GM, Salvemini D. A role for nitric oxide-mediated peroxynitrite formation in a model of endotoxin induced shock. $\mathrm{J}$ Pharmacol Exp Ther. In press

266. Cuzzocrea S, Mazzon E, Dugo L, Barbera A, Centorrino T, Ciccolo A, Fonti MT, Caputi AP. Inducible nitric oxide synthase knockout mice exhibit resistance to the multiple organ failure induced by zymosan. Shock 2001;16:51-58. [PubMed: 11442316]

267. Cuzzocrea S, Mazzon E, Dugo L, Caputi AP, Aston K, Riley DP, Salvemini D. Protective effects of a new stable, highly active SOD mimetic, M40401 in splanchnic artery occlusion and reperfusion. Br J Pharmacol 2001;132:19-29. [PubMed: 11156557]

268. Cuzzocrea S, Mazzon E, Dugo L, Caputi AP, Riley DP, Salvemini D. Protective effects of M40403, a superoxide dismutase mimetic, in a rodent model of colitis. Eur J Pharmacol 2001;432:79-89. [PubMed: 11734191]

269. Cuzzocrea S, Mazzon E, Dugo L, Di Paola R, Caputi AP, Salvemini D. Superoxide: a key player in hypertension. FASEB J 2004;18:94-101. [PubMed: 14718390]

270. Cuzzocrea S, McDonald MC, Mazzon E, Filipe HM, Centorrino T, Lepore V, Terranova ML, Ciccolo A, Caputi AP, Thiemermann C. Beneficial effects of tempol, a membrane-permeable radical scavenger, on the multiple organ failure induced by zymosan in the rat. Crit Care Med 2001;29:102-111. [PubMed: 11176168]

271. Cuzzocrea S, McDonald MC, Mazzon E, Filipe HM, Costantino G, Caputi AP, Thiemermann C. Beneficial effects of tempol, a membrane-permeable radical scavenger, in a rodent model of splanchnic artery occlusion and reperfusion. Shock 2000;14:150-156. [PubMed: 10947159]

272. Cuzzocrea S, Misko TP, Costantino G, Mazzon E, Micali A, Caputi AP, Macarthur H, Salvemini D. Beneficial effects of peroxynitrite decomposition catalyst in a rat model of splanchnic artery occlusion and reperfusion. FASEB J 2000;14:1061-1072. [PubMed: 10834927]

273. Cuzzocrea S, Riley DP, Caputi AP, Salvemini D. Antioxidant therapy: a new pharmacological approach in shock, inflammation, and ischemia/reperfusion injury. Pharmacol Rev 2001;53:135159. [PubMed: 11171943]

274. Cuzzocrea S, Zingarelli B, Caputi AP. Role of constitutive nitric oxide synthase and peroxynitrite production in a rat model of splanchnic artery occlusion shock. Life Sci 1998;63:789-799. [PubMed: 9740316]

275. Cuzzocrea S, Zingarelli B, Caputi AP. Role of peroxynitrite and poly (ADP-ribosyl) synthetase activation in cardiovascular derangement induced by zymosan in the rat. Life Sci 1998;63:923933. [PubMed: 9747893]

276. Cuzzocrea S, Zingarelli B, Costantino G, Caputi AP. Protective effect of melatonin in a non-septic shock model induced by zymosan in the rat. J Pineal Res 1998;25:24-33. [PubMed: 9694401]

277. Cuzzocrea S, Zingarelli B, Costantino G, Szabo A, Salzman AL, Caputi AP, Szabo C. Beneficial effects of 3-aminobenzamide, an inhibitor of poly(ADP-ribose) synthetase in a rat model of splanchnic artery occlusion and reperfusion. Br J Pharmacol 1997;121:1065-1074. [PubMed: 9249240]

278. Cuzzocrea S, Zingarelli B, O’Connor M, Salzman AL, Szabo C. Effect of L-buthionine-(S,R)sulphoximine, an inhibitor of gamma-glutamylcysteine synthetase on peroxynitrite- and endotoxic shock-induced vascular failure. Br J Pharmacol 1998;123:525-537. [PubMed: 9504394]

280. Da Ros R, Assaloni R, Ceriello A. Antioxidant therapy in diabetic complications: what is new? Curr Vasc Pharmacol 2004;2:335-341. [PubMed: 15320813]

281. Dai ZK, Tan MS, Chai CY, Yeh JL, Chou SH, Chiu CC, Jeng AY, Chen IJ, Wu JR. Upregulation of endothelial nitric oxide synthase and endothelin-1 in pulmonary hypertension secondary to heart failure in aorta-banded rats. Pediatr Pulmonol 2004;37:249-256. [PubMed: 14966819]

282. Daiber A, Herold S, Schoneich C, Namgaladze D, Peterson JA, Ullrich V. Nitration and inactivation of cytochrome P450BM-3 by peroxynitrite. Stopped-flow measurements prove ferryl intermediates. Eur J Biochem 2000;267:6729-6739. [PubMed: 11082183]

283. Daiber A, Mulsch A, Hink U, Mollnau H, Warnholtz A, Oelze M, Munzel T. The oxidative stress concept of nitrate tolerance and the antioxidant properties of hydralazine. Am J Cardiol 2005;96:25i-36i. [PubMed: 15979427] 
284. Daiber A, Schoneich C, Schmidt P, Jung C, Ullrich V. Auto-catalytic nitration of P450CAM by peroxynitrite. J Inorg Biochem 2000;81:213-220. [PubMed: 11051566]

285. Dairou J, Atmane N, Rodrigues-Lima F, Dupret JM. Peroxynitrite irreversibly inactivates the human xenobiotic-metabolizing enzyme arylamine $N$-acetyltransferase 1 (NAT1) in human breast cancer cells: a cellular and mechanistic study. J Biol Chem 2004;279:7708-7714. [PubMed: 14672957]

286. Damy T, Ratajczak P, Shah AM, Camors E, Marty I, Hasenfuss G, Marotte F, Samuel JL, Heymes C. Increased neuronal nitric oxide synthase-derived NO production in the failing human heart. Lancet 2004;363:1365-1367. [PubMed: 15110495]

287. Darley-Usmar VM, Hogg N, O'Leary VJ, Wilson MT, Moncada S. The simultaneous generation of superoxide and nitric oxide can initiate lipid peroxidation in human low density lipoprotein. Free Radic Res Commun 1992;17:9-20. [PubMed: 1332919]

288. Davidovic L, Vodenicharov M, Affar EB, Poirier GG. Importance of poly(ADP-ribose) glycohydrolase in the control of poly-(ADP-ribose) metabolism. Exp Cell Res 2001;268:7-13. [PubMed: 11461113]

289. Davignon J, Ganz P. Role of endothelial dysfunction in atherosclerosis. Circulation 2004;109(Suppl III):III-27-III-32. [PubMed: 15198963]

290. Davis B, Zou MH. CD40 ligand-dependent tyrosine nitration of prostacyclin synthase in vivo. Circulation 2005;112:2184-2192. [PubMed: 16186418]

291. Davis RJ. Signal transduction by the JNK group of MAP kinases. Cell 2000;103:239-252. [PubMed: 11057897]

292. Dawson TM, Bredt DS, Fotuhi M, Hwang PM, Snyder SH. Nitric oxide synthase and neuronal NADPH diaphorase are identical in brain and peripheral tissues. Proc Natl Acad Sci USA 1991;88:7797-7801. [PubMed: 1715581]

293. Dawson TM, Dawson VL. Molecular pathways of neurodegeneration in Parkinson's disease. Science 2003;302:819-822. [PubMed: 14593166]

294. Dawson VL, Dawson TM. Deadly conversations: nuclear-mitochondrial cross-talk. J Bioenerget Biomembr 2004;36:287-294.

295. Dawson VL, Dawson TM, London ED, Bredt DS, Snyder SH. Nitric oxide mediates glutamate neurotoxicity in primary cortical cultures. Proc Natl Acad Sci USA 1991;88:6368-6371. [PubMed: 1648740]

296. De Groote MA, Ochsner UA, Shiloh MU, Nathan C, McCord JM, Dinauer MC, Libby SJ, VazquezTorres A, Xu Y, Fang FC. Periplasmic superoxide dismutase protects Salmonella from products of phagocyte NADPH-oxidase and nitric oxide synthase. Proc Natl Acad Sci USA 1997;94:1399714001. [PubMed: 9391141]

297. De la Monte SM, Lu BX, Sohn YK, Etienne D, Kraft J, Ganju N, Wands JR. Aberrant expression of nitric oxide synthase III in Alzheimer's disease: relevance to cerebral vasculopathy and neurodegeneration. Neurobiol Aging 2000;21:309-319. [PubMed: 10867216]

298. De la Monte SM, Sohn YK, Etienne D, Kraft J, Wands JR. Role of aberrant nitric oxide synthase-3 expression in cerebrovascular degeneration and vascular-mediated injury in Alzheimer's disease. Ann NY Acad Sci 2000;903:61-71. [PubMed: 10818490]

298a. d'Emmanuele di Villa Bianca R, Marzocco S, Di Paola R, Autore G, Pinto A, Cuzzocrea S, Sorrentino R. Melatonin prevents lipopolysaccharide-induced hyporeactivity in rat. J Pineal Res 2004;36:146-154. [PubMed: 15009504]

299. De Murcia G, Menissier de Murcia J. Poly(ADP-ribose) polymerase: a molecular nick sensor. Trends Biochem Sci 1994;19:172-176. [PubMed: 8016868]

300. Deckel AW, Duffy JD. Vasomotor hyporeactivity in the anterior cerebral artery during motor activation in Huntington's disease patients. Brain Res 2000;872:258-261. [PubMed: 10924705]

301. Deckel AW, Gordinier A, Nuttal D, Tang V, Kuwada C, Freitas R, Gary KA. Reduced activity and protein expression of NOS in R6/2 HD transgenic mice: effects of L-NAME on symptom progression. Brain Res 2001;919:70-81. [PubMed: 11689164]

302. Deckel AW, Volmer P, Weiner R, Gary KA, Covault J, Sasso D, Schmerler N, Watts D, Yan Z, Abeles I. Dietary arginine alters time of symptom onset in Huntington's disease transgenic mice. Brain Res 2000;875:187-195. [PubMed: 10967315] 
303. Deeb RS, Hao G, Gross SS, Laine M, Qiu JH, Resnick B, Barbar EJ, Hajjar DP, Upmacis RK. Heme catalyzes Tyr 385 nitration and inactivation of prostaglandin $\mathrm{H} 2$ synthase-1 by peroxynitrite. J Lipid Res 2006;47:898-911. [PubMed: 16470026]

304. Deeb RS, Shen H, Gamss C, Gavrilova T, Summers BD, Kraemer R, Hao G, Gross SS, Laine M, Maeda N, Hajjar DP, Upmacis RK. Inducible nitric oxide synthase mediates prostaglandin h2 synthase nitration and suppresses eicosanoid production. Am J Pathol 2006;168:349-362. [PubMed: 16400036]

305. Deguchi T, Yoshioka M. L-Arginine identified as an endogenous activator for soluble guanylate cyclase from neuroblastoma cells. J Biol Chem 1982;257:10147-10151. [PubMed: 6125510]

306. Dejardin E, Droin NM, Delhase M, Haas E, Cao Y, Makris C, Li ZW, Karin M, Ware CF, Green DR. The lymphotoxin-beta receptor induces different patterns of gene expression via two NFkappaB pathways. Immunity 2002;17:525-535. [PubMed: 12387745]

307. Delaney CA, Green MH, Lowe JE, Green IC. Endogenous nitric oxide induced by interleukin-1 beta in rat islets of Langerhans and HIT-T15 cells causes significant DNA damage as measured by the “comet" assay. FEBS Lett 1993;333:291-295. [PubMed: 8224196]

308. Delaney CA, Pavlovic D, Hoorens A, Pipeleers DG, Eizirik DL. Cytokines induce deoxyribonucleic acid strand breaks and apoptosis in human pancreatic islet cells. Endocrinology 1997;138:2610 2614. [PubMed: 9165055]

309. Delaney CA, Tyrberg B, Bouwens L, Vaghef H, Hellman B, Eizirik DL. Sensitivity of human pancreatic islets to peroxynitrite-induced cell dysfunction and death. FEBS Lett 1996;394:300-306. [PubMed: 8830662]

310. Denicola A, Freeman BA, Trujillo M, Radi R. Peroxynitrite reaction with carbon dioxide/ bicarbonate: kinetics and influence on peroxynitrite-mediated oxidations. Arch Biochem Biophys 1996;333:49-58. [PubMed: 8806753]

311. Denicola A, Radi R. Peroxynitrite and drug-dependent toxicity. Toxicology 2005;208:273-288. [PubMed: 15691591]

312. Denicola A, Souza JM, Radi R. Diffusion of peroxynitrite across erythrocyte membranes. Proc Natl Acad Sci USA 1998;95:3566-3571. [PubMed: 9520406]

313. Depre C, Havaux X, Renkin J, Vanoverschelde JL, Wijns W. Expression of inducible nitric oxide synthase in human coronary atherosclerotic plaque. Cardiovasc Res 1999;41:465-472. [PubMed: 10341846]

314. Dhar A, Brindley JM, Stark C, Citro ML, Keefer LK, Colburn NH. Nitric oxide does not mediate but inhibits transformation and tumor phenotype. Mol Cancer Ther 2003;2:1285-1293. [PubMed: 14707269]

315. Dhar A, Kaundal RK, Sharma SS. Neuroprotective effects of FeTMPyP: a peroxynitrite decomposition catalyst in global cerebral ischemia model in gerbils. Pharmacol Res. In press

316. Di Filippo C, Cuzzocrea S, Marfella R, Fabbroni V, Scollo G, Berrino L, Giugliano D, Rossi F, D'Amico M. M40403 prevents myocardial injury induced by acute hyperglycaemia in perfused rat heart. Eur J Pharmacol 2004;497:65-74. [PubMed: 15321736]

317. Di Stasi AM, Mallozzi C, Macchia G, Maura G, Petrucci TC, Minetti M. Peroxynitrite affects exocytosis and SNARE complex formation and induces tyrosine nitration of synaptic proteins. $\mathrm{J}$ Neurochem 2002;82:420-429. [PubMed: 12124443]

318. Di Stasi AM, Mallozzi C, Macchia G, Petrucci TC, Minetti M. Peroxynitrite induces tryosine nitration and modulates tyrosine phosphorylation of synaptic proteins. J Neurochem 1999;73:727735. [PubMed: 10428070]

319. Dickhout JG, Hossain GS, Pozza LM, Zhou J, Lhotak S, Austin RC. Peroxynitrite causes endoplasmic reticulum stress and apoptosis in human vascular endothelium: implications in atherogenesis. Arterioscler Thromb Vasc Biol 2005;25:2623-2629. [PubMed: 16210571]

320. Digerness SB, Harris KD, Kirklin JW, Urthaler F, Viera L, Beckman JS, Darley-Usmar V. Peroxynitrite irreversibly decreases diastolic and systolic function in cardiac muscle. Free Radic Biol Med 1999;27:1386-1392. [PubMed: 10641733]

321. Dijkstra G, Moshage H, van Dullemen HM, de Jager-Krikken A, Tiebosch AT, Kleibeuker JH, Jansen PL, van Goor H. Expression of nitric oxide synthases and formation of nitrotyrosine and 
reactive oxygen species in inflammatory bowel disease. J Pathol 1998;186:416-421. [PubMed: 10209492]

322. Dikopoulos N, Nussler AK, Liptay S, Bachem M, Reinshagen M, Stiegler M, Schmid RM, Adler $\mathrm{G}$, Weidenbach $\mathrm{H}$. Inhibition of nitric oxide synthesis by aminoguanidine increases intestinal damage in the acute phase of rat TNB-colitis. Eur J Clin Invest 2001;31:234-239. [PubMed: 11264651]

323. Dimmeler S, Lottspeich F, Brüne B. Nitric oxide causes ADP-ribosylation and inhibition of glyceraldehyde-3-phosphate dehydrogenase. J Biol Chem 1992;267:16771-16774. [PubMed: 1512218]

324. Ding X, Hiraku Y, Ma N, Kato T, Saito K, Nagahama M, Semba R, Kuribayashi K, Kawanishi S. Inducible nitric oxide synthase-dependent DNA damage in mouse model of inflammatory bowel disease. Cancer Sci 2005;96:157-163. [PubMed: 15771618]

325. Ding Y, Gonick HC, Vaziri ND, Liang K, Wei L. Lead-induced hypertension. III. Increased hydroxyl radical production. Am J Hypertens 2001;14:169-173. [PubMed: 11243309]

326. Dirnagl U, Iadecola C, Moskowitz MA. Pathobiology of ischaemic stroke: an integrated view. Trends Neurosci 1999;22:391-397. [PubMed: 10441299]

327. Dobrian AD, Schriver SD, Prewitt RL. Role of angiotensin II and free radicals in blood pressure regulation in a rat model of renal hypertension. Hypertension 2001;38:361-366. [PubMed: 11566905]

328. Dong C, Davis RJ, Flavell RA. MAP kinases in the immune response. Annu Rev Immunol 2002;20:55-72. [PubMed: 11861597]

329. Drapier JD, Hibbs JB. Differentiation of murine macrophages to express nonspecific cytotoxicity for tumor cells results in L-arginine-dependent inhibition of mitochondrial iron-sulfur enzymes in the macrophage effector cells. J Immunol 1988;140:2829-2838. [PubMed: 2451695]

330. Drayton DL, Bonizzi G, Ying X, Liao S, Karin M, Ruddle NH. IкB kinase complex $\alpha$ kinase activity controls chemokine and high endothelial venule gene expression in lymph nodes and nasalassociated lymphoid tissue. J Immunol 2004;173:6161-6168. [PubMed: 15528353]

331. Drel VR, Pacher P, Stevens MJ, Obrosova IG. Aldose reductase inhibition counteracts nitrosative stress and poly(ADP-ribose) polymerase activation in diabetic rat kidney and high-glucose-exposed human mesangial cells. Free Radic Biol Med 2006;40:1454-1465. [PubMed: 16631535]

332. Du X, Matsumura T, Edelstein D, Rossetti L, Zsengeller Z, Szabo C, Brownlee M. Inhibition of GAPDH activity by poly-(ADP-ribose) polymerase activates three major pathways of hyperglycemic damage in endothelial cells. J Clin Invest 2003;112:1049-1057. [PubMed: 14523042]

333. Du Y, Smith MA, Miller CM, Kern TS. Diabetes-induced nitrative stress in the retina, and correction by aminoguanidine. J Neurochem 2002;80:771-779. [PubMed: 11948240]

334. Ducrocq C, Blanchard B, Pignatelli B, Ohshima H. Peroxynitrite: an endogenous oxidizing and nitrating agent. Cell Mol Life Sci 1999;55:1068-1077. [PubMed: 10442088]

335. Duranski MR, Greer JJ, Dejam A, Jaganmohan S, Hogg N, Langston W, Patel RP, Yet SF, Wang X, Kevil CG, Gladwin MT, Lefer DJ. Cytoprotective effects of nitrite during in vivo ischemiareperfusion of the heart and liver. J Clin Invest 2005;115:1232-1240. [PubMed: 15841216]

336. East SJ, Garthwaite J. NMDA receptor activation in the rat hippocampus induces cyclic GMP formation through the L-arginine-nitric oxide pathway. Neurosci Lett 1991;123:17-19. [PubMed: 1648186]

337. Ebadi M, Sharma SK. Peroxynitrite and mitochondrial dysfunction in the pathogenesis of Parkinson's disease. Antioxid Redox Signal 2003;5:319-335. [PubMed: 12880486]

338. Ebadi M, Sharma SK, Ghafourifar P, Brown-Borg H, El Refaey H. Peroxynitrite in the pathogenesis of Parkinson's disease and the neuroprotective role of metallothioneins. Methods Enzymol 2005;396:276-298. [PubMed: 16291239]

339. Eberhardt RT, Forgione MA, Cap A, Leopold JA, Rudd MA, Trolliet M, Heydrick S, Stark R, Klings ES, Moldovan NI, Yaghoubi M, Goldschmidt-Clermont PJ, Farber HW, Cohen R, Loscalzo J. Endothelial dysfunction in a murine model of mild hyperhomocyst(e)inemia. J Clin Invest 2000;106:483-491. [PubMed: 10953023] 
340. Egan LJ, Eckmann L, Greten FR, Chae S, Li ZW, Myhre GM, Robine S, Karin M, Kagnoff MF. IkappaB-kinasebeta-dependent NF-kappaB activation provides radioprotection to the intestinal epithelium. Proc Natl Acad Sci USA 2004;101:2452-2457. [PubMed: 14983030]

341. Egan TM, Hoffmann SC, Sevala M, Sadoff JD, Schlidt SA. Nitroglycerin reperfusion reduces ischemia-reperfusion injury in non-heart-beating donor lungs. J Heart Lung Transplant 2006;25:110-119. [PubMed: 16399539]

342. Egi K, Conrad NE, Bedynek A, Ferdinandy P, Reichart B, Wildhirt SM. Improvement of cardiac allograft function by $1400 \mathrm{~W}$, a highly selective inducible nitric oxide synthase inhibitor and superoxide dismutase: role of peroxynitrite. J Heart Lung Transplant 2001;20:154. [PubMed: 11250221]

343. Eid HM, Lyberg T, Larsen J, Arnesen H, Seljeflot I. Reactive oxygen species generation by leukocytes in populations at risk for atherosclerotic disease. Scand J Clin Lab Invest 2002;62:431439. [PubMed: 12469898]

344. Eizirik DL, Delaney CA, Green MH, Cunningham JM, Thorpe JR, Pipeleers DG, Hellerstrom C, Green IC. Nitric oxide donors decrease the function and survival of human pancreatic islets. Mol Cell Endocrinol 1996;118:71-83. [PubMed: 8735593]

345. Eizirik DL, Sandler S, Welsh N, Cetkovic-Cvrlje M, Nieman A, Geller DA, Pipeleers DG, Bendtzen $\mathrm{K}$, Hellerstrom C. Cytokines suppress human islet function irrespective of their effects on nitric oxide generation. J Clin Invest 1994;93:1968-1974. [PubMed: 7514190]

346. El Kossi MM, Zakhary MM. Oxidative stress in the context of acute cerebrovascular stroke. Stroke 2000;31:1889-1892. [PubMed: 10926952]

347. El-Dwairi Q, Comtois A, Guo Y, Hussain SN. Endotoxin-induced skeletal muscle contractile dysfunction: contribution of nitric oxide synthases. Am J Physiol Cell Physiol 1998;274:C770 C779.

348. Eliasson MJ, Huang Z, Ferrante RJ, Sasamata M, Molliver ME, Snyder SH, Moskowitz MA. Neuronal nitric oxide synthase activation and peroxynitrite formation in ischemic stroke linked to neural damage. J Neurosci 1999;19:5910-5918. [PubMed: 10407030]

349. Eliasson MJ, Sampei K, Mandir AS, Hurn PD, Traystman RJ, Bao J, Pieper A, Wang ZQ, Dawson TM, Snyder SH, Dawson VL. Poly(ADP-ribose) polymerase gene disruption renders mice resistant to cerebral ischemia. Nat Med 1997;3:1089-1095. [PubMed: 9334719]

350. Eligini S, Habib A, Lebret M, Creminon C, Levy-Toledano S, Maclouf J. Induction of cyclooxygenase- 2 in human endothelial cells by SIN-1 in the absence of prostaglandin production. $\mathrm{Br} \mathrm{J}$ Pharmacol 2001;133:1163-1171. [PubMed: 11487528]

351. Ellis EA, Guberski DL, Hutson B, Grant MB. Time course of NADH oxidase, inducible nitric oxide synthase and peroxynitrite in diabetic retinopathy in the BBZ/WOR rat. Nitric Oxide 2002;6:295304. [PubMed: 12009847]

352. El-Remessy AB, Abou-Mohamed G, Caldwell RW, Caldwell RB. High glucose-induced tyrosine nitration in endothelial cells: role of eNOS uncoupling and aldose reductase activation. Invest Ophthalmol Vis Sci 2003;44:3135-3143. [PubMed: 12824263]

353. El-Remessy AB, Bartoli M, Platt DH, Fulton D, Caldwell RB. Oxidative stress inactivates VEGF survival signaling in retinal endothelial cells via PI 3-kinase tyrosine nitration. J Cell Sci 2005;118:243-252. [PubMed: 15615788]

354. El-Remessy AB, Behzadian MA, Abou-Mohamed G, Franklin T, Caldwell RW, Caldwell RB. Experimental diabetes causes breakdown of the blood-retina barrier by a mechanism involving tyrosine nitration and increases in expression of vascular endothelial growth factor and urokinase plasminogen activator receptor. Am J Pathol 2003;162:1995-2004. [PubMed: 12759255]

355. El-Sokkary GH, Reiter RJ, Cuzzocrea S, Caputi AP, Hassanein AF, Tan DX. Role of melatonin in reduction of lipid peroxidation and peroxynitrite formation in non-septic shock induced by zymosan. Shock 1999;12:402-408. [PubMed: 10565615]

356. Emerit J, Edeas M, Bricaire F. Neurodegenerative diseases and oxidative stress. Biomed Pharmacother 2004;58:39-46. [PubMed: 14739060]

357. Endres M, Laufs U, Huang Z, Nakamura T, Huang P, Moskowitz MA, Liao JK. Stroke protection by 3-hydroxy-3-methyl-glutaryl (HMG)-CoA reductase inhibitors mediated by endothelial nitric oxide synthase. Proc Natl Acad Sci USA 1998;95:8880-8885. [PubMed: 9671773] 
358. Endres M, Laufs U, Liao JK, Moskowitz MA. Targeting eNOS for stroke protection. Trends Neurosci 2004;27:283-289. [PubMed: 15111011]

359. Endres M, Scott G, Namura S, Salzman AL, Huang PL, Moskowitz MA, Szabo C. Role of peroxynitrite and neuronal nitric oxide synthase in the activation of poly(ADP-ribose) synthetase in a murine model of cerebral ischemia-reperfusion. Neurosci Lett 1998;248:41-44. [PubMed: 9665659]

360. Endres M, Wang ZQ, Namura S, Waeber C, Moskowitz MA. Ischemic brain injury is mediated by the activation of poly(ADP-ribose)polymerase. J Cereb Blood Flow Metab 1997;17:1143-1151. [PubMed: 9390645]

361. Erdelyi K, Bakondi E, Gergely P, Szabo C, Virag L. Pathophysiologic role of oxidative stressinduced poly(ADP-ribose) polymerase-1 activation: focus on cell death and transcriptional regulation. Cell Mol Life Sci 2005;62:751-759. [PubMed: 15868400]

362. Eriksen JL, Wszolek Z, Petrucelli L. Molecular pathogenesis of Parkinson disease. Arch Neurol 2005;62:353-357. [PubMed: 15767499]

363. Esaki T, Hayashi T, Muto E, Kano H, Kumar TN, Asai Y, Sumi D, Iguchi A. Expression of inducible nitric oxide synthase and Fas/Fas ligand correlates with the incidence of apoptotic cell death in atheromatous plaques of human coronary arteries. Nitric Oxide 2000;4:561-571. [PubMed: 11139364]

364. Esberg LB, Ren J. Role of nitric oxide, tetrahydrobiopterin and peroxynitrite in glucose toxicityassociated contractile dysfunction in ventricular myocytes. Diabetologia 2003;46:1419-1427. [PubMed: 12898015]

365. Escobales N, Crespo MJ. Oxidative-nitrosative stress in hypertension. Curr Vasc Pharmacol 2005;3:231-246. [PubMed: 16026320]

366. Estevez AG, Crow JP, Sampson JB, Reiter C, Zhuang Y, Richardson GJ, Tarpey MM, Barbeito L, Beckman JS. Induction of nitric oxide-dependent apoptosis in motor neurons by zinc-deficient superoxide dismutase. Science 1999;286:2498-2500. [PubMed: 10617463]

367. Estevez AG, Radi R, Barbeito L, Shin JT, Thompson JA, Beckman JS. Peroxynitrite-induced cytotoxicity in PC12 cells: evidence for an apoptotic mechanism differentially modulated by neurotrophic factors. J Neurochem 1995;65:1543-1550. [PubMed: 7561848]

368. Estevez AG, Sampson JB, Zhuang YX, Spear N, Richardson GJ, Crow JP, Tarpey MM, Barbeito L, Beckman JS. Liposome-delivered superoxide dismutase prevents nitric oxide-dependent motor neuron death induced by trophic factor withdrawal. Free Radic Biol Med 2000;28:437-446. [PubMed: 10699756]

369. Estevez AG, Spear N, Manuel SM, Barbeito L, Radi R, Beckman JS. Role of endogenous nitric oxide and peroxynitrite formation in the survival and death of motor neurons in culture. Prog Brain Res 1998;118:269-280. [PubMed: 9932448]

370. Estevez AG, Spear N, Manuel SM, Radi R, Henderson CE, Barbeito L, Beckman JS. Nitric oxide and superoxide contribute to motor neuron apoptosis induced by trophic factor deprivation. $\mathrm{J}$ Neurosci 1998;18:923-931. [PubMed: 9437014]

371. Estevez, AG.; Spear, N.; Pelluffo, H.; Kamaid, A.; Barbeito, L.; Beckman, JS. Examining apoptosis in cultured cells after exposure to nitric oxide and peroxynitrite. In: Packer, L., editor. Nitric Oxide: Biological and Antioxidant Activities. San Diego, CA: Academic; 1998. p. 393-402.

372. Evgenov OV, Pacher P, Schmidt PM, Hasko G, Schmidt HHW, Stasch JP. Nitric oxide-independent stimulators and activators of soluble guanylate cyclase. Nat Rev Drug Discov 2006;5:755-768. [PubMed: 16955067]

373. Evgenov OV, Liaudet L. Role of nitrosative stress and activation of poly(ADP-ribose) polymerase-1 in cardiovascular failure associated with septic and hemorrhagic shock. Curr Vasc Pharmacol 2005;3:293-299. [PubMed: 16026325]

374. Fabian RH, DeWitt DS, Kent TA. In vivo detection of superoxide anion production by the brain using a cytochrome $c$ electrode. J Cereb Blood Flow Metab 1995;15:242-247. [PubMed: 7860658]

375. Facchinetti F, Sasaki M, Cutting FB, Zhai P, MacDonald JE, Reif D, Beal MF, Huang PL, Dawson TM, Gurney ME, Dawson VL. Lack of involvement of neuronal nitric oxide synthase in the pathogenesis of a transgenic mouse model of familial amyotrophic lateral sclerosis. Neuroscience 1999;90:1483-1492. [PubMed: 10338314] 
376. Faden AI. Neuroprotection and traumatic brain injury: theoretical option or realistic proposition. Curr Opin Neurol 2002;15:707-712. [PubMed: 12447109]

377. Farrell AJ, Blake DR, Palmer RM, Moncada S. Increased concentrations of nitrite in synovial fluid and serum samples suggest increased nitric oxide synthesis in rheumatic diseases. Ann Rheum Dis 1992;51:1219-1222. [PubMed: 1466599]

378. Fehsel K, Jalowy A, Qi S, Burkart V, Hartmann B, Kolb H. Islet cell DNA is a target of inflammatory attack by nitric oxide. Diabetes 1993;42:496-500. [PubMed: 8432420]

379. Feigl EO. EDRF-a protective factor? Nature 1988;331:490-491. [PubMed: 3257547]

380. Feihl F, Waeber B, Liaudet L. Is nitric oxide overproduction the target of choice for the management of septic shock? Pharmacol Ther 2001;91:179-213. [PubMed: 11744067]

381. Feletou M, Vanhoutte PM. Endothelial dysfunction: a multifaceted disorder. Am J Physiol Heart Circ Physiol 2006;291:H985-H1002. [PubMed: 16632549]

382. Feliciello A, Gottesman ME, Avvedimento EV. cAMP-PKA signaling to the mitochondria: protein scaffolds, mRNA and phosphatases. Cell Signal 2005;17:279-287. [PubMed: 15567059]

383. Feng Q, Lu X, Jones DL, Shen J, Arnold JM. Increased inducible nitric oxide synthase expression contributes to myocardial dysfunction and higher mortality after myocardial infarction in mice. Circulation 2001;104:700-704. [PubMed: 11489778]

384. Ferdinandy P, Danial H, Ambrus I, Rothery RA, Schulz R. Peroxynitrite is a major contributor to cytokine-induced myocardial contractile failure. Circ Res 2000;87:241-247. [PubMed: 10926876]

385. Ferdinandy P, Panas D, Schulz R. Peroxynitrite contributes to spontaneous loss of cardiac efficiency in isolated working rat hearts. Am J Physiol Heart Circ Physiol 1999;276:H1861-H1867.

386. Ferdinandy P, Schulz R. Nitric oxide, superoxide, and peroxynitrite in myocardial ischaemiareperfusion injury and preconditioning. Br J Pharmacol 2003;138:532-543. [PubMed: 12598407]

387. Feron O, Belhassen L, Kobzik L, Smith TW, Kelly RA, Michel T. Endothelial nitric oxide synthase targeting to caveolae. J Biol Chem 1996;271:22810-22814. [PubMed: 8798458]

388. Ferrante RJ, Shinobu LA, Schulz JB, Matthews RT, Thomas CE, Kowall NW, Gurney ME, Beal MF. Increased 3-nitrotyrosine and oxidative damage in mice with a human copper/zinc superoxide dismutase mutation. Ann Neurol 1997;42:326-334. [PubMed: 9307254]

389. Ferreiro CR, Chagas AC, Carvalho MH, Dantas AP, Scavone C, Souza LC, Buffolo E, da Luz PL. Expression of inducible nitric oxide synthase is increased in patients with heart failure due to ischemic disease. Braz J Med Biol Res 2004;37:1313-1320. [PubMed: 15334196]

390. Ferriero DM, Holtzman DM, Black SM, Sheldon RA. Neonatal mice lacking neuronal nitric oxide synthase are less vulnerable to hypoxic-ischemic injury. Neurobiol Dis 1996;3:64-71. [PubMed: 9173913]

391. Filep JG, Beauchamp M, Baron C, Paquette Y. Peroxynitrite mediates IL-8 gene expression and production in lipopolysaccharide-stimulated human whole blood. J Immunol 1998;161:5656-5662. [PubMed: 9820546]

392. Fink MP. Bench-to-bedside review: cytopathic hypoxia. Crit Care 2002;6:491-499. [PubMed: 12493070]

393. Fink MP. Role of reactive oxygen and nitrogen species in acute respiratory distress syndrome. Curr Opin Crit Care 2002;8:6-11. [PubMed: 12205400]

394. Finkel T, Holbrook NJ. Oxidants, oxidative stress and the biology of ageing. Nature 2000;408:239247. [PubMed: 11089981]

395. Fiocchi C. Inflammatory bowel disease: etiology and pathogenesis. Gastroenterology 1998;115:182-205. [PubMed: 9649475]

396. Firestein GS. Evolving concepts of rheumatoid arthritis. Nature 2003;423:356-361. [PubMed: 12748655]

397. Fiser SM, Tribble CG, Long SM, Kaza AK, Cope JT, Laubach VE, Kern JA, Kron IL. Lung transplant reperfusion injury involves pulmonary macrophages and circulating leukocytes in a biphasic response. J Thorac Cardiovasc Surg 2001;121:1069-1075. [PubMed: 11385373]

398. Fletcher DS, Widmer WR, Luell S, Christen A, Orevillo C, Shah S, Visco D. Therapeutic administration of a selective inhibitor of nitric oxide synthase does not ameliorate the chronic 
inflammation and tissue damage associated with adjuvant-induced arthritis in rats. J Pharmacol Exp Ther 1998;284:714-721. [PubMed: 9454819]

399. Flodstrom M, Tyrberg B, Eizirik DL, Sandler S. Reduced sensitivity of inducible nitric oxide synthase-deficient mice to multiple low-dose streptozotocin-induced diabetes. Diabetes 1999;48:706-713. [PubMed: 10102685]

400. Floris R, Piersma SR, Yang G, Jones P, Wever R. Interaction of myeloperoxidase with peroxynitrite. A comparison with lactoperoxidase, horseradish peroxidase and catalase. Eur J Biochem 1993;215:767-775. [PubMed: 8394811]

401. Fontijn A, Sadadell AJ, Ronco RJ. Homogeneous chemiluminescent measurement of nitric oxide with ozone. Anal Chem 1970;42:575-578.

402. Forbes JM, Cooper ME, Thallas V, Burns WC, Thomas MC, Brammar GC, Lee F, Grant SL, Burrell LA, Jerums G, Osicka TM. Reduction of the accumulation of advanced glycation end products by ACE inhibition in experimental diabetic nephropathy. Diabetes 2002;51:3274-3282. [PubMed: 12401719]

403. Forsmark-Andree P, Persson B, Radi R, Dallner G, Ernster L. Oxidative modification of nicotinamide nucleotide transhydrogenase in submitochondrial particles: effect of endogenous ubiquinol. Arch Biochem Biophys 1996;336:113-120. [PubMed: 8951041]

404. Forster C, Clark HB, Ross ME, Iadecola C. Inducible nitric oxide synthase expression in human cerebral infarcts. Acta Neuropathol 1999;97:215-220. [PubMed: 10090667]

405. Forstermann U. Endothelial NO synthase as a source of NO and superoxide. Eur J Clin Pharmacol 2006;62(Suppl 13):5-12.

406. Forstermann, U. NO synthases. In: Offermanns, S.; Rosenthal, W., editors. Encyclopedic Reference of Molecular Pharmacology. Berlin: Springer-Verlag; 2003. p. 653-658.

407. Forstermann U, Munzel T. Endothelial nitric oxide synthase in vascular disease: from marvel to menace. Circulation 2006;113:1708-1714. [PubMed: 16585403]

408. Fortenberry JD, Owens ML, Brown MR, Atkinson D, Brown LA. Exogenous nitric oxide enhances neutrophil cell death and DNA fragmentation. Am J Respir Cell Mol Biol 1998;18:421-428. [PubMed: 9490660]

409. Francia P, delli Gatti C, Bachschmid M, Martin-Padura I, Savoia C, Migliaccio E, Pelicci PG, Schiavoni M, Luscher TF, Volpe M, Cosentino F. Deletion of p66shc gene protects against agerelated endothelial dysfunction. Circulation 2004;110:2889-2895. [PubMed: 15505103]

410. Fridovich I. Quantitative aspects of the production of superoxide anion radical by milk xanthine oxidase. J Biol Chem 1970;245:4053-4057. [PubMed: 5496991]

411. Frostell CG, Blomqvist H, Hedenstierna G, Lundberg J, Zapol WM. Inhaled nitric oxide selectively reverses human hypoxic pulmonary vasoconstriction without causing systemic vasodilation. Anesthesiology 1993;78:427-435. [PubMed: 8457043]

412. Frostell D, Fratacci MD, Wain JC, Jones R, Zapol WM. Inhaled nitric oxide. A selective pulmonary vasodilator reversing hypoxic pulmonary vasoconstriction. Circulation 1991;83:2038-2047. [PubMed: 2040056]

413. Frustaci A, Kajstura J, Chimenti C, Jakoniuk I, Leri A, Maseri A, Nadal-Ginard B, Anversa P. Myocardial cell death in human diabetes. Circ Res 2000;87:1123-1132. [PubMed: 11110769]

414. Fu Y, Sies H, Lei XG. Opposite roles of selenium-dependent glutathione peroxidase-1 in superoxide generator diquat- and peroxynitrite-induced apoptosis and signaling. J Biol Chem 2001;276:4300443009. [PubMed: 11562367]

415. Fujigaki H, Saito K, Lin F, Fujigaki S, Takahashi K, Martin BM, Chen CY, Masuda J, Kowalak J, Takikawa O, Seishima M, Markey SP. Nitration and inactivation of IDO by peroxynitrite. J Immunol 2006;176:372-379. [PubMed: 16365430]

416. Fukui T, Ishizaka N, Rajagopalan S, Laursen JB, Capers Qt Taylor WR, Harrison DG, de Leon H, Wilcox JN, Griendling KK. p22phox mRNA expression and NADPH oxidase activity are increased in aortas from hypertensive rats. Circ Res 1997;80:45-51. [PubMed: 8978321]

417. Fukumoto K, Pierro A, Zammit VA, Spitz L, Eaton S. Tyrosine nitration of carnitine palmitoyl transferase I during endotoxaemia in suckling rats. Biochim Biophys Acta 2004;1683:1-6.

[PubMed: 15238213] 
418. Fukumura D, Kashiwagi S, Jain RK. The role of nitric oxide in tumour progression. Nat Rev Cancer 2006;6:521-534. [PubMed: 16794635]

419. Fukushima T, Tawara T, Isobe A, Hojo N, Shiwaku K, Yamane Y. Radical formation site of cerebral complex I and Parkinson's disease. J Neurosci Res 1995;42:385-390. [PubMed: 8583507]

420. Fukuyama N, Takebayashi Y, Hida M, Ishida H, Ichimori K, Nakazawa H. Clinical evidence of peroxynitrite formation in chronic renal failure patients with septic shock. Free Radic Biol Med 1997;22:771-774. [PubMed: 9119244]

421. Funakoshi H, Kubota T, Kawamura N, Machida Y, Feldman AM, Tsutsui H, Shimokawa H, Takeshita A. Disruption of inducible nitric oxide synthase improves beta-adrenergic inotropic responsiveness but not the survival of mice with cytokine-induced cardiomyopathy. Circ Res 2002;90:959-965. [PubMed: 12016261]

422. Furchgott RF, Zawadzki JV. The obligatory role of endothelial cells in the relaxation of arterial smooth muscle by acetylcholine. Nature 1980;288:373-376. [PubMed: 6253831]

423. Gahm C, Danilov A, Holmin S, Wiklund PN, Brundin L, Mathiesen T. Reduced neuronal injury after treatment with $N^{\mathrm{G}}$-nitro-L-arginine methyl ester (L-NAME) or 2-sulfo-phenyl- $N$-tert-butyl nitrone (S-PBN) following experimental brain contusion. Neurosurgery 2005;57:1272-1281. [PubMed: 16331176]

424. Galkina SI, Dormeneva EV, Bachschmid M, Pushkareva MA, Sud'ina GF, Ullrich V. Endotheliumleukocyte interactions under the influence of the superoxide-nitrogen monoxide system. Med Sci Monit 2004;10:BR307-316. [PubMed: 15328475]

425. Galley HF, Davies MJ, Webster NR. Xanthine oxidase activity and free radical generation in patients with sepsis syndrome. Crit Care Med 1996;24:1649-1653. [PubMed: 8874300]

426. Gally JA, Montague PR, Reeke GN Jr, Edelman GM. The NO hypothesis: possible effects of a shortlived, rapidly diffusible signal in the development and function of the nervous system. Proc Natl Acad Sci USA 1990;87:3547-3551. [PubMed: 2333301]

427. Galpern WR, Matthews RT, Beal MF, Isacson O. NGF attenuates 3-nitrotyrosine formation in a 3NP model of Huntington's disease. Neuroreport 1996;7:2639-2642. [PubMed: 8981438]

428. Gao CQ, Sawicki G, Suarez-Pinzon WL, Csont T, Wozniak M, Ferdinandy P, Schulz R. Matrix metalloproteinase-2 mediates cytokine-induced myocardial contractile dysfunction. Cardiovasc Res 2003;57:426-433. [PubMed: 12566115]

429. Gao F, Yao CL, Gao E, Mo QZ, Yan WL, McLaughlin R, Lopez BL, Christopher TA, Ma XL. Enhancement of glutathione cardioprotection by ascorbic acid in myocardial reperfusion injury. J Pharmacol Exp Ther 2002;301:543-550. [PubMed: 11961055]

430. Garcia Soriano F, Virag L, Jagtap P, Szabo E, Mabley JG, Liaudet L, Marton A, Hoyt DG, Murthy KG, Salzman AL, Southan GJ, Szabo C. Diabetic endothelial dysfunction: the role of poly(ADPribose) polymerase activation. Nat Med 2001;7:108-113. [PubMed: 11135624]

431. Gardian G, Vecsei L. Huntington's disease: pathomechanism and therapeutic perspectives. J Neural Transm 2004;111:1485-1494. [PubMed: 15480847]

432. Gardner CR, Laskin JD, Dambach DM, Sacco M, Durham SK, Bruno MK, Cohen SD, Gordon MK, Gerecke DR, Zhou P, Laskin DL. Reduced hepatotoxicity of acetaminophen in mice lacking inducible nitric oxide synthase: potential role of tumor necrosis factor-alpha and interleukin-10. Toxicol Appl Pharmacol 2002;184:27-36. [PubMed: 12392966]

433. Garthwaite J. Glutamate, nitric oxide and cell-cell signalling in the nervous system. Trends Neurosci 1991;14:75-82.

434. Garthwaite J, Boulton CL. Nitric oxide signaling in the central nervous system. Annu Rev Physiol 1995;57:683-706. [PubMed: 7539993]

435. Garthwaite J, Charles SL, Chess-Williams R. Endothelium-derived relaxing factor release on activation of NMDA receptors suggests role as intercellular messenger in the brain. Nature 1988;336:385-388. [PubMed: 2904125]

436. Garthwaite J, Sotham E, Boulton CL, Nielsen EB, Schmidt K, Mayer B. Potent and selective inhibition of nitric oxide-sensitive guanylyl cyclase by $1 H[1,2,4]$ oxadiazolo[4,3- $a$ ]quinoxalin-1one. Biochem Pharmacol 1996;48:184-188. 
437. Gatto EM, Riobo NA, Carreras MC, Chernavsky A, Rubio A, Satz ML, Poderoso JJ. Overexpression of neutrophil neuronal nitric oxide synthase in Parkinson's disease. Nitric Oxide 2000;4:534-539. [PubMed: 11020342]

438. Gaut JP, Yeh GC, Tran HD, Byun J, Henderson JP, Richter GM, Brennan ML, Lusis AJ, Belaaouaj A, Hotchkiss RS, Heinecke JW. Neutrophils employ the myeloperoxidase system to generate antimicrobial brominating and chlorinating oxidants during sepsis. Proc Natl Acad Sci USA 2001;98:11961-11966. [PubMed: 11593004]

439. Gealekman O, Abassi Z, Rubinstein I, Winaver J, Binah O. Role of myocardial inducible nitric oxide synthase in contractile dysfunction and beta-adrenergic hyporesponsiveness in rats with experimental volume-overload heart failure. Circulation 2002;105:236-243. [PubMed: 11790707]

440. Geha RM, Rebrin I, Chen K, Shih JC. Substrate and inhibitor specificities for human monoamine oxidase A and B are influenced by a single amino acid. J Biol Chem 2001;276:9877-9882. [PubMed: 11134050]

441. Gentleman SM, Leclercq PD, Moyes L, Graham DI, Smith C, Griffin WS, Nicoll JA. Long-term intracerebral inflammatory response after traumatic brain injury. Forensic Sci Int 2004;146:97-104. [PubMed: 15542269]

442. Ghosh S, Karin M. Missing pieces in the NF-kappaB puzzle. Cell 2002;109(Suppl):S81-S96. [PubMed: 11983155]

443. Giasson BI, Duda JE, Murray IV, Chen Q, Souza JM, Hurtig HI, Ischiropoulos H, Trojanowski JQ, Lee VM. Oxidative damage linked to neurodegeneration by selective alpha-synuclein nitration in synucleinopathy lesions. Science 2000;290:985-989. [PubMed: 11062131]

444. Gilad E, Zingarelli B, Salzman AL, Szabo C. Protection by inhibition of poly(ADP-ribose) synthetase against oxidant injury in cardiac myoblasts In vitro. J Mol Cell Cardiol 1997;29:25852597. [PubMed: 9299380]

445. Giovannoni G. Cerebrospinal fluid and serum nitric oxide metabolites in patients with multiple sclerosis. Mult Scler 1998;4:27-30. [PubMed: 9532589]

446. Giovannoni G, Heales SJ, Land JM, Thompson EJ. The potential role of nitric oxide in multiple sclerosis. Mult Scler 1998;4:212-216. [PubMed: 9762676]

447. Giovannoni G, Heales SJ, Silver NC, O’Riordan J, Miller RF, Land JM, Clark JB, Thompson EJ. Raised serum nitrate and nitrite levels in patients with multiple sclerosis. J Neurol Sci 1997;145:7781. [PubMed: 9073032]

448. Giovannoni G, Silver NC, O’Riordan J, Miller RF, Heales SJ, Land JM, Elliot M, Feldmann M, Miller DH, Thompson EJ. Increased urinary nitric oxide metabolites in patients with multiple sclerosis correlates with early and relapsing disease. Mult Scler 1999;5:335-341. [PubMed: 10516777]

449. Gloire G, Legrand-Poels S, Piette J. NF-kB activation by reactive oxygen species: fifteen years later. Biochem Pharmacol. In press

450. Go YM, Patel RP, Maland MC, Park H, Beckman JS, Darley-Usmar VM, Jo H. Evidence for peroxynitrite as a signaling molecule in flow-dependent activation of c-Jun NH(2)-terminal kinase. Am J Physiol Heart Circ Physiol 1999;277:H1647-H1653.

451. Goldfarb RD, Marton A, Szabo E, Virag L, Salzman AL, Glock D, Akhter I, McCarthy R, Parrillo JE, Szabo C. Protective effect of a novel, potent inhibitor of poly(adenosine 5'-diphosphate-ribose) synthetase in a porcine model of severe bacterial sepsis. Crit Care Med 2002;30:974-980. [PubMed: 12006790]

452. Goldstein S, Czapski G, Lind J, Merenyi G. Effect of *NO on the decomposition of peroxynitrite: reaction of $\mathrm{N}_{2} \mathrm{O}_{3}$ with ONOO. Chem Res Toxicol 1999;12:132-136. [PubMed: 10027789]

453. Golfetti R, VanDyke K, Rork T, Spiler N, Merrill G. Acetaminophen in the post-ischemia reperfused myocardium. Exp Biol Med 2002;227:1031-1037.

454. Goligorsky MS, Brodsky SV, Noiri E. Nitric oxide in acute renal failure: NOS versus NOS. Kidney Int 2002;61:855-861. [PubMed: 11849438]

455. Gomez CB, del Valle HF, Bertolotti A, Negroni JA, Cuniberti L, Martinez V, Osses J, Laguens RP, Favaloro RR. Effects of short-term inhaled nitric oxide on interleukin-8 release after single-lung transplantation in pigs. J Heart Lung Transplant 2005;24:714-722. [PubMed: 15949732] 
456. Good PF, Hsu A, Werner P, Perl DP, Olanow CW. Protein nitration in Parkinson's disease. J Neuropathol Exp Neurol 1998;57:338-342. [PubMed: 9600227]

457. Good PF, Werner P, Hsu A, Olanow CW, Perl DP. Evidence of neuronal oxidative damage in Alzheimer's disease. Am J Pathol 1996;149:21-28. [PubMed: 8686745]

458. Gopalakrishna R, Jaken S. Protein kinase C signaling and oxidative stress. Free Radic Biol Med 2000;28:1349-1361. [PubMed: 10924854]

459. Gorg B, Bidmon HJ, Keitel V, Foster N, Goerlich R, Schliess F, Haussinger D. Inflammatory cytokines induce protein tyrosine nitration in rat astrocytes. Arch Biochem Biophys 2006;449:104114. [PubMed: 16579953]

460. Gorg B, Wettstein M, Metzger S, Schliess F, Haussinger D. Lipopolysaccharide-induced tyrosine nitration and inactivation of hepatic glutamine synthetase in the rat. Hepatology 2005;41:10651073. [PubMed: 15830392]

461. Gori T, Parker JD. Nitrate tolerance: a unifying hypothesis. Circulation 2002;106:2510-2513. [PubMed: 12417551]

462. Gouge RC, Marshburn P, Gordon BE, Nunley W, Huet-Hudson YM. Nitric oxide as a regulator of embryonic development. Biol Reprod 1998;58:875-879. [PubMed: 9546715]

463. Gow AJ, Duran D, Malcolm S, Ischiropoulos H. Effects of peroxynitrite-induced protein modifications on tyrosine phosphorylation and degradation. FEBS Lett 1996;385:63-66. [PubMed: 8641468]

464. Gow AJ, Farkouh CR, Munson DA, Posencheg MA, Ischiropoulos H. Biological significance of nitric oxide-mediated protein modifications. Am J Physiol Lung Cell Mol Physiol 2004;287:L262L268. [PubMed: 15246980]

465. Graham A, Hogg N, Kalyanaraman B, O’Leary V, Darley-Usmar V, Moncada S. Peroxynitrite modification of low-density lipoprotein leads to recognition by the macrophage scavenger receptor. FEBS Lett 1993;330:181-185. [PubMed: 8365489]

466. Grandati M, Verrecchia C, Revaud ML, Allix M, Boulu RG, Plotkine M. Calcium-independent NOsynthase activity and nitrites/nitrates production in transient focal cerebral ischaemia in mice. $\mathrm{Br} \mathrm{J}$ Pharmacol 1997;122:625-630. [PubMed: 9375957]

467. Granger DN, Rutili G, McCord JM. Superoxide radicals in feline intestinal ischemia. Gastroenterology 1981;81:22-29. [PubMed: 6263743]

468. Grasemann H, Drazen JM, Deykin A, Israel E, DeSanctis GT, Pillari A, Yandava CN. Simple tandem repeat polymorphisms in the neuronal nitric oxide synthase gene in different ethnic populations. Hum Hered 1999;49:139-141. [PubMed: 10364677]

469. Graves JE, Bates JN, Kooy NW, Lewis SJ. Vasodilator actions of the endothelium-derived relaxing factor L-S-nitrosocysteine in anaesthetized rats are markedly diminished by peroxynitrite. Clin Exp Pharmacol Physiol 2005;32:1137-1141. [PubMed: 16445582]

470. Graves JE, Kooy NW, Lewis SJ. L-beta,beta-Dimethylcysteine attenuates the haemodynamic responses elicited by systemic injections of peroxynitrite in anaesthetized rats. Br J Pharmacol. In press

471. Graves JE, Lewis SJ, Kooy NW. Loss of K+ATP-channel-mediated vasodilation after induction of tachyphylaxis to peroxynitrite. J Cardiovasc Pharmacol 2005;46:646-652. [PubMed: 16220072]

472. Graves JE, Lewis SJ, Kooy NW. Peroxynitrite-mediated vasorelaxation: evidence against the formation of circulating S-nitrosothiols. Am J Physiol Heart Circ Physiol 1998;274:H1001-H1008.

473. Graves JE, Lewis SJ, Kooy NW. Role of ATP-sensitive $\mathrm{K}^{+}$-channels in hemodynamic effects of peroxynitrite in anesthetized rats. J Cardiovasc Pharmacol 2005;46:653-659. [PubMed: 16220073]

474. Green IC, Cunningham JM, Delaney CA, Elphick MR, Mabley JG, Green MH. Effects of cytokines and nitric oxide donors on insulin secretion, cyclic GMP and DNA damage: relation to nitric oxide production. Biochem Soc Trans 1994;22:30-37. [PubMed: 7515831]

475. Green LC, Ruiz de Luzuriaga K, Wagner DA, Rand W, Istfan N, Young VR, Tannenbaum SR. Nitrate biosynthesis in man. Proc Natl Acad Sci USA 1981;78:7764-7768. [PubMed: 6950416]

476. Greenacre SA, Ischiropoulos H. Tyrosine nitration: localisation, quantification, consequences for protein function and signal transduction. Free Radic Res 2001;34:541-581. [PubMed: 11697033] 
477. Greten FR, Eckmann L, Greten TF, Park JM, Li ZW, Egan LJ, Kagnoff MF, Karin M. IKKbeta links inflammation and tumorigenesis in a mouse model of colitis-associated cancer. Cell 2004;118:285296. [PubMed: 15294155]

478. Griendling KK, Sorescu D, Ushio-Fukai M. NAD(P)H oxidase: role in cardiovascular biology and disease. Circ Res 2000;86:494-501. [PubMed: 10720409]

479. Griffith TM, Edwards DH, Davies RL, Harrison TJ, Evans KT. EDRF coordinates the behaviour of vascular resistance vessels. Nature 1987;329:442-445. [PubMed: 3498901]

480. Grishko V, Pastukh V, Solodushko V, Gillespie M, Azuma J, Schaffer S. Apoptotic cascade initiated by angiotensin II in neonatal cardiomyocytes: role of DNA damage. Am J Physiol Heart Circ Physiol 2003;285:H2364-H2372. [PubMed: 12919932]

481. Groenendaal F, Lammers H, Smit D, Nikkels PG. Nitrotyrosine in brain tissue of neonates after perinatal asphyxia. Arch Dis Child Fetal Neonatal Ed. In press

482. Grover AK, Samson SE. Effect of superoxide radical on $\mathrm{Ca}^{2+}$ pumps of coronary artery. Am J Physiol Cell Physiol 1988;255:C297-C303.

483. Grover AK, Samson SE, Robinson S, Kwan CY. Effects of peroxynitrite on sarcoplasmic reticulum $\mathrm{Ca}^{2+}$ pump in pig coronary artery smooth muscle. Am J Physiol Cell Physiol 2003;284:C294-C301. [PubMed: 12529249]

484. Gryglewski RJ, Palmer RM, Moncada S. Superoxide anion is involved in the breakdown of endothelium-derived vascular relaxing factor. Nature 1986;320:454-456. [PubMed: 3007998]

485. Gu X, El-Remessy AB, Brooks SE, Al-Shabrawey M, Tsai NT, Caldwell RB. Hyperoxia induces retinal vascular endothelial cell apoptosis through formation of peroxynitrite. Am J Physiol Cell Physiol 2003;285:C546-C554. [PubMed: 12736139]

486. Gu ZY, Ling YL, Xu XH, Meng AH, Li SJ. Effect of peroxynitrite on the reactivity of rabbit pulmonary arteries in vitro. Sheng Li Xue Bao 2003;55:469-474. [PubMed: 12937830]

487. Gu ZY, Ling YL, Xu XH, Zhu TN, Cong B. Endogenous peroxynitrite mediates lipopolysaccharideinduced injury in cultured pulmonary artery endothelial cells. Sheng Li Xue Bao 2003;55:475-480. [PubMed: 12937831]

488. Gugliucci A. Human plasminogen is highly susceptible to peroxynitrite inactivation. Clin Chem Lab Med 2003;41:1064-1068. [PubMed: 12964815]

489. Guidarelli A, Fiorani M, Cantoni O. Enhancing effects of intracellular ascorbic acid on peroxynitriteinduced U937 cell death are mediated by mitochondrial events resulting in enhanced sensitivity to peroxynitrite-dependent inhibition of complex III and formation of hydrogen peroxide. Biochem J 2004;378:959-966. [PubMed: 14627438]

490. Guittet O, Ducastel B, Salem JS, Henry Y, Rubin H, Lemaire G, Lepoivre M. Differential sensitivity of the tyrosyl radical of mouse ribonucleotide reductase to nitric oxide and peroxynitrite. J Biol Chem 1998;273:22136-22144. [PubMed: 9705359]

491. Guix FX, Uribesalgo I, Coma M, Munoz FJ. The physiology and pathophysiology of nitric oxide in the brain. Prog Neurobiol 2005;76:126-152. [PubMed: 16115721]

492. Guo W, Adachi T, Matsui R, Xu S, Jiang B, Zou MH, Kirber M, Lieberthal W, Cohen RA. Quantitative assessment of tyrosine nitration of manganese superoxide dismutase in angiotensin IIinfused rat kidney. Am J Physiol Heart Circ Physiol 2003;285:H1396-H1403. [PubMed: 12791589]

493. Gurney ME, Pu H, Chiu AY, Dal Canto MC, Polchow CY, Alexander DD, Caliendo J, Hentati A, Kwon YW, Deng HX, Chen W, Zhai P, Sufit RL, Siddique T. Motor neuron degeneration in mice that express a human $\mathrm{Cu}, \mathrm{Zn}$ superoxide dismutase mutation. Science 1994;264:1772-1775. [PubMed: 8209258]

494. Gursoy-Ozdemir Y, Bolay H, Saribas O, Dalkara T. Role of endothelial nitric oxide generation and peroxynitrite formation in reperfusion injury after focal cerebral ischemia. Stroke 2000;31:19741981. [PubMed: 10926966]

495. Gursoy-Ozdemir Y, Can A, Dalkara T. Reperfusion-induced oxidative/nitrative injury to neurovascular unit after focal cerebral ischemia. Stroke 2004;35:1449-1453. [PubMed: 15073398]

496. Gutierrez-Martin Y, Martin-Romero FJ, Inesta-Vaquera FA, Gutierrez-Merino C, Henao F. Modulation of sarcoplasmic reticulum $\mathrm{Ca}(2+)$-ATPase by chronic and acute exposure to peroxynitrite. Eur J Biochem 2004;271:2647-2657. [PubMed: 15206930] 
497. Gutkind JS. Regulation of mitogen-activated protein kinase signaling networks by G protein-coupled receptors. Sci STKE 2000 2000:RE1.

498. Guy RA, Maguire GF, Crandall I, Connelly PW, Kain KC. Characterization of peroxynitriteoxidized low density lipoprotein binding to human CD36. Atherosclerosis 2001;155:19-28. [PubMed: 11223422]

499. Guyenet PG. The sympathetic control of blood pressure. Nat Rev Neurosci 2006;7:335-346. [PubMed: 16760914]

500. Guzik TJ, Mussa S, Gastaldi D, Sadowski J, Ratnatunga C, Pillai R, Channon KM. Mechanisms of increased vascular superoxide production in human diabetes mellitus: role of $\mathrm{NAD}(\mathrm{P}) \mathrm{H}$ oxidase and endothelial nitric oxide synthase. Circulation 2002;105:1656-1662. [PubMed: 11940543]

501. Ha HC. Defective transcription factor activation for proinflammatory gene expression in poly(ADPribose) polymerase 1-deficient glia. Proc Natl Acad Sci USA 2004;101:5087-5092. [PubMed: 15041747]

502. Ha HC, Hester LD, Snyder SH. Poly(ADP-ribose) polymerase-1 dependence of stress-induced transcription factors and associated gene expression in glia. Proc Natl Acad Sci USA 2002;99:32703275. [PubMed: 11854472]

503. Ha HC, Snyder SH. Poly(ADP-ribose) polymerase is a mediator of necrotic cell death by ATP depletion. Proc Natl Acad Sci USA 1999;96:13978-13982. [PubMed: 10570184]

504. Hadjivassiliou V, Green MH, James RF, Swift SM, Clayton HA, Green IC. Insulin secretion, DNA damage, and apoptosis in human and rat islets of Langerhans following exposure to nitric oxide, peroxynitrite, and cytokines. Nitric Oxide 1998;2:429-441. [PubMed: 10342486]

505. Hafezi-Moghadam A, Simoncini T, Yang Z, Limbourg FP, Plumier JC, Rebsamen MC, Hsieh CM, Chui DS, Thomas KL, Prorock AJ, Laubach VE, Moskowitz MA, French BA, Ley K, Liao JK. Acute cardiovascular protective effects of corticosteroids are mediated by non-transcriptional activation of endothelial nitric oxide synthase. Nat Med 2002;8:473-479. [PubMed: 11984591]

506. Hajjar I, Kotchen JM, Kotchen TA. Hypertension: trends in prevalence, incidence, and control. Annu Rev Public Health 2006;27:465-490. [PubMed: 16533126]

507. Hall ED, Detloff MR, Johnson K, Kupina NC. Peroxynitrite-mediated protein nitration and lipid peroxidation in a mouse model of traumatic brain injury. J Neurotrauma 2004;21:9-20. [PubMed: 14987461]

508. Hall ED, Kupina NC, Althaus JS. Peroxynitrite scavengers for the acute treatment of traumatic brain injury. Ann NY Acad Sci 1999;890:462-468. [PubMed: 10668450]

509. Hamilton CA, Brosnan MJ, McIntyre M, Graham D, Dominiczak AF. Superoxide excess in hypertension and aging: a common cause of endothelial dysfunction. Hypertension 2001;37:529534. [PubMed: 11230330]

510. Hamilton ML, Van Remmen H, Drake JA, Yang H, Guo ZM, Kewitt K, Walter CA, Richardson A. Does oxidative damage to DNA increase with age? Proc Natl Acad Sci USA 2001;98:10469-10474. [PubMed: 11517304]

511. Han D, Canali R, Garcia J, Aguilera R, Gallaher TK, Cadenas E. Sites and mechanisms of aconitase inactivation by peroxynitrite: modulation by citrate and glutathione. Biochemistry 2005;44:1198611996. [PubMed: 16142896]

512. Hanada M, Feng J, Hemmings BA. Structure, regulation and function of PKB/AKT - a major therapeutic target. Biochim Biophys Acta 2004;1697:3-16. [PubMed: 15023346]

513. Hanauer SB, Sandborn WJ, Rutgeerts P, Fedorak RN, Lukas M, MacIntosh D, Panaccione R, Wolf D, Pollack P. Human anti-tumor necrosis factor monoclonal antibody (adalimumab) in Crohn's disease: the CLASSIC-I trial. Gastroenterology 2006;130:323-333. [PubMed: 16472588]

514. Hansson GK. Inflammation, atherosclerosis, and coronary artery disease. N Engl J Med 2005;352:1685-1695. [PubMed: 15843671]

515. Haqqani AS, Kelly JF, Birnboim HC. Selective nitration of histone tyrosine residues in vivo in mutatect tumors. J Biol Chem 2002;277:3614-3621. [PubMed: 11723112]

516. Hare JM, Stamler JS. NO/redox disequilibrium in the failing heart and cardiovascular system. J Clin Invest 2005;115:509-517. [PubMed: 15765132]

517. Harman D. Aging: a theory based on free radical and radiation chemistry. J Gerontol 1956;11:298300. [PubMed: 13332224] 
518. Harrison D, Griendling KK, Landmesser U, Hornig B, Drexler H. Role of oxidative stress in atherosclerosis. Am J Cardiol 2003;91:7A-11A.

519. Haskew RE, Mongin AA, Kimelberg HK. Peroxynitrite enhances astrocytic volume-sensitive excitatory amino acid release via a src tyrosine kinase-dependent mechanism. J Neurochem 2002;82:903-912. [PubMed: 12358796]

520. Hassa PO, Hottiger MO. The functional role of poly(ADP-ribose) polymerase 1 as novel coactivator of NF-kappaB in inflammatory disorders. Cell Mol Life Sci 2002;59:1534-1553. [PubMed: 12440774]

521. Hatake K, Kakishita E, Wakabayashi I, Sakiyama N, Hishida S. Effect of aging on endotheliumdependent vascular relaxation of isolated human basilar artery to thrombin and bradykinin. Stroke 1990;21:1039-1043. [PubMed: 2114673]

522. Hattori N, Mizuno Y. Pathogenetic mechanisms of parkin in Parkinson's disease. Lancet 2004;364:722-724. [PubMed: 15325839]

523. Hausladen A, Fridovich I. Superoxide and peroxynitrite inactivate aconitases, but nitric oxide does not. J Biol Chem 1994;269:29405-29408. [PubMed: 7961919]

524. Hayashi T, Sumi D, Juliet PA, Matsui-Hirai H, Asai-Tanaka Y, Kano H, Fukatsu A, Tsunekawa T, Miyazaki A, Iguchi A, Ignarro LJ. Gene transfer of endothelial NO synthase, but not eNOS plus inducible NOS, regressed atherosclerosis in rabbits. Cardiovasc Res 2004;61:339-351. [PubMed: 14736551]

525. Hayashi Y, Sawa Y, Fukuyama N, Miyamoto Y, Takahashi T, Nakazawa H, Matsuda H. Leukocytedepleted terminal blood cardioplegia provides superior myocardial protective effects in association with myocardium-derived nitric oxide and peroxynitrite production for patients undergoing prolonged aortic cross-clamping for more than 120 minutes. J Thorac Cardiovasc Surg 2003;126:1813-1821. [PubMed: 14688692]

526. Hayashi Y, Sawa Y, Ohtake S, Fukuyama N, Nakazawa H, Matsuda H. Peroxynitrite formation from human myocardium after ischemia-reperfusion during open heart operation. Ann Thorac Surg 2001;72:571-576. [PubMed: 11515899]

527. Hayat SA, Patel B, Khattar RS, Malik RA. Diabetic cardiomyopathy: mechanisms, diagnosis and treatment. Clin Sci 2004;107:539-557. [PubMed: 15341511]

528. Haynes V, Elfering S, Traaseth N, Giulivi C. Mitochondrial nitric-oxide synthase: enzyme expression, characterization, and regulation. J Bioenerget Biomembr 2004;36:341-346.

529. Haywood GA, Tsao PS, von der Leyen HE, Mann MJ, Keeling PJ, Trindade PT, Lewis NP, Byrne CD, Rickenbacher PR, Bishopric NH, Cooke JP, McKenna WJ, Fowler MB. Expression of inducible nitric oxide synthase in human heart failure. Circulation 1996;93:1087-1094. [PubMed: 8653828]

530. Hazen SL, Zhang R, Shen Z, Wu W, Podrez EA, MacPherson JC, Schmitt D, Mitra SN, Mukhopadhyay C, Chen Y, Cohen PA, Hoff HF, Abu-Soud HM. Formation of nitric oxide-derived oxidants by myeloperoxidase in monocytes: pathways for monocyte-mediated protein nitration and lipid peroxidation in vivo. Circ Res 1999;85:950-958. [PubMed: 10559142]

531. He K, Nukada H, McMorran PD, Murphy MP. Protein carbonyl formation and tyrosine nitration as markers of oxidative damage during ischaemia-reperfusion injury to rat sciatic nerve. Neuroscience 1999;94:909-916. [PubMed: 10579583]

532. Heales SJ, Bolanos JP, Stewart VC, Brookes PS, Land JM, Clark JB. Nitric oxide, mitochondria and neurological disease. Biochim Biophys Acta 1999;1410:215-228. [PubMed: 10076028]

533. Heck DE, Kagan VE, Shvedova AA, Laskin JD. An epigrammatic (abridged) recounting of the myriad tales of astonishing deeds and dire consequences pertaining to nitric oxide and reactive oxygen species in mitochondria with an ancillary missive concerning the origins of apoptosis. Toxicology 2005;208:259-271. [PubMed: 15691590]

534. Heger J, Godecke A, Flogel U, Merx MW, Molojavyi A, Kuhn-Velten WN, Schrader J. Cardiacspecific overexpression of inducible nitric oxide synthase does not result in severe cardiac dysfunction. Circ Res 2002;90:93-99. [PubMed: 11786524]

535. Heitzer T, Wenzel U, Hink U, Krollner D, Skatchkov M, Stahl RA, MacHarzina R, Brasen JH, Meinertz T, Munzel T. Increased NAD(P)H oxidase-mediated superoxide production in re- 
novascular hypertension: evidence for an involvement of protein kinase C. Kidney Int 1999;55:252_ 260. [PubMed: 9893134]

536. Hellberg CB, Boggs SE, Lapetina EG. Phosphatidylinositol 3-kinase is a target for protein tyrosine nitration. Biochem Biophys Res Commun 1998;252:313-317. [PubMed: 9826526]

537. Henrotin YE, Bruckner P, Pujol JP. The role of reactive oxygen species in homeostasis and degradation of cartilage. Osteoarthritis Cartilage 2003;11:747-755. [PubMed: 13129694]

538. Hensley K, Maidt ML, Yu Z, Sang H, Markesbery WR, Floyd RA. Electrochemical analysis of protein nitrotyrosine and dityrosine in the Alzheimer brain indicates region-specific accumulation. J Neurosci 1998;18:8126-8132. [PubMed: 9763459]

539. Herceg Z, Wang Z. Functions of poly(ADP-ribose) polymerase (PARP) in DNA repair, genomic integrity and cell death. Mutat Res 2001;477:97-110. [PubMed: 11376691]

540. Herold S, Exner M, Boccini F. The mechanism of the peroxynitrite-mediated oxidation of myoglobin in the absence and presence of carbon dioxide. Chem Res Toxicol 2003;16:390-402. [PubMed: 12641440]

541. Herskowitz A, Choi S, Ansari AA, Wesselingh S. Cytokine mRNA expression in postischemic/ reperfused myocardium. Am J Pathol 1995;146:419-428. [PubMed: 7856752]

542. Hibbs JB Jr, Taintor RR, Vavrin Z. Macrophage cytotoxicity: role of L-arginine deminiase and imino nitrogen oxidation to nitrite. Science 1987;235:473-476. [PubMed: 2432665]

543. Hibbs JB Jr, Taintor RR, Vavrin Z, Rachlin EM. Nitric oxide: a cytotoxic activated macrophage effector molecule. Biochem Biophys Res Commun 1988;157:87-94. [PubMed: 3196352]

544. Hierholzer C, Billiar TR. Molecular mechanisms in the early phase of hemorrhagic shock. Langenbecks Arch Surg 2001;386:302-308. [PubMed: 11466573]

545. Higashi Y, Oshima T, Ozono R, Matsuura H, Kajiyama G. Aging and severity of hypertension attenuate endothelium-dependent renal vascular relaxation in humans. Hypertension 1997;30:252258. [PubMed: 9260989]

546. Hill KE, Zollinger LV, Watt HE, Carlson NG, Rose JW. Inducible nitric oxide synthase in chronic active multiple sclerosis plaques: distribution, cellular expression and association with myelin damage. J Neuroimmunol 2004;151:171-179. [PubMed: 15145615]

547. Hillier BJ, Christopherson KS, Prehoda KE, Bredt DS, Lim WA. Unexpected modes of PDZ domain scaffolding revealed by structure of nNOS-syntrophin complex. Science 1999;284:812-815. [PubMed: 10221915]

548. Hines IN, Harada H, Bharwani S, Pavlick KP, Hoffman JM, Grisham MB. Enhanced post-ischemic liver injury in iNOS-deficient mice: a cautionary note. Biochem Biophys Res Commun 2001;284:972-976. [PubMed: 11409889]

549. Hink U, Oelze M, Kolb P, Bachschmid M, Zou MH, Daiber A, Mollnau H, August M, Baldus S, Tsilimingas N, Walter U, Ullrich V, Munzel T. Role for peroxynitrite in the inhibition of prostacyclin synthase in nitrate tolerance. J Am Coll Cardiol 2003;42:1826-1834. [PubMed: 14642695]

550. Hirabayashi H, Takizawa S, Fukuyama N, Nakazawa H, Shinohara Y. 7-Nitroindazole attenuates nitrotyrosine formation in the early phase of cerebral ischemia-reperfusion in mice. Neurosci Lett 1999;268:111-113. [PubMed: 10400091]

551. Hirabayashi H, Takizawa S, Fukuyama N, Nakazawa H, Shinohara Y. N-methyl-D-aspartate receptor antagonist reduces nitrotyrosine formation in caudate-putamen in rat focal cerebral ischemia-reperfusion. Neurosci Lett 2001;299:159-161. [PubMed: 11166962]

552. Hirabayashi H, Takizawa S, Fukuyama N, Nakazawa H, Shinohara Y. Nitrotyrosine generation via inducible nitric oxide synthase in vascular wall in focal ischemia-reperfusion. Brain Res 2000;852:319-325. [PubMed: 10678758]

553. Hoeldtke RD, Bryner KD, McNeill DR, Hobbs GR, Baylis C. Peroxynitrite versus nitric oxide in early diabetes. Am J Hypertens 2003;16:761-766. [PubMed: 12944035]

554. Hoeldtke RD, Bryner KD, McNeill DR, Hobbs GR, Riggs JE, Warehime SS, Christie I, Ganser G, Van Dyke K. Nitrosative stress, uric acid, and peripheral nerve function in early type 1 diabetes. Diabetes 2002;51:2817-2825. [PubMed: 12196476] 
555. Hoeldtke RD, Bryner KD, McNeill DR, Warehime SS, Van Dyke K, Hobbs G. Oxidative stress and insulin requirements in patients with recent-onset type 1 diabetes. J Clin Endocrinol Metab 2003;88:1624-1628. [PubMed: 12679448]

556. Hoffmann J, Haendeler J, Aicher A, Rossig L, Vasa M, Zeiher AM, Dimmeler S. Aging enhances the sensitivity of endothelial cells toward apoptotic stimuli: important role of nitric oxide. Circ Res 2001;89:709-715. [PubMed: 11597994]

557. Hogaboam CM, Jacobson K, Collins SM, Blennerhassett MG. The selective beneficial effects of nitric oxide inhibition in experimental colitis. Am J Physiol Gastrointest Liver Physiol 1995;268:G673-G684.

558. Hogg N, Darley-Usmar VM, Graham A, Moncada S. Peroxynitrite and atherosclerosis. Biochem Soc Trans 1993;21:358-362. [PubMed: 8359496]

559. Hogg N, Darley-Usmar VM, Wilson MT, Moncada S. Production of hydroxyl radicals from the simultaneous generation of superoxide and nitric oxide. Biochem J 1992;281:419-424. [PubMed: 1310595]

560. Hogg N, Kalyanaraman B. Nitric oxide and lipid peroxidation. Biochim Biophys Acta 1999;1411:378-384. [PubMed: 10320670]

561. Hogg N, Kalyanaraman B, Joseph J, Struck A, Parthasarathy S. Inhibition of low-density lipoprotein oxidation by nitric oxide. Potential role in atherogenesis. FEBS Lett 1993;334:170-174. [PubMed: 8224243]

562. Hokari R, Kato S, Matsuzaki K, Kuroki M, Iwai A, Kawaguchi A, Nagao S, Miyahara T, Itoh K, Sekizuka E, Nagata H, Ishii H, Miura S. Reduced sensitivity of inducible nitric oxide synthasedeficient mice to chronic colitis. Free Radic Biol Med 2001;31:153-163. [PubMed: 11440827]

563. Holm L, Cassidy JD, Carroll LJ, Borg J. Summary of the WHO collaborating centre for neurotrauma task force on mild traumatic brain injury. J Rehabil Med 2005;37:137-141. [PubMed: 16040469]

564. Holscher C. Possible causes of Alzheimer's disease: amyloid fragments, free radicals, and calcium homeostasis. Neurobiol Dis 1998;5:129-141. [PubMed: 9848086]

565. Hong HJ, Hsiao G, Cheng TH, Yen MH. Supplemention with tetrahydrobiopterin suppresses the development of hypertension in spontaneously hypertensive rats. Hypertension 2001;38:10441048. [PubMed: 11711495]

566. Hooper DC, Bagasra O, Marini JC, Zborek A, Ohnishi ST, Kean R, Champion JM, Sarker AB, Bobroski L, Farber JL, Akaike T, Maeda H, Koprowski H. Prevention of experimental allergic encephalomyelitis by targeting nitric oxide and peroxynitrite: implications for the treatment of multiple sclerosis. Proc Natl Acad Sci USA 1997;94:2528-2533. [PubMed: 9122229]

567. Hooper DC, Spitsin S, Kean RB, Champion JM, Dickson GM, Chaudhry I, Koprowski H. Uric acid, a natural scavenger of peroxynitrite, in experimental allergic encephalomyelitis and multiple sclerosis. Proc Natl Acad Sci USA 1998;95:675-680. [PubMed: 9435251]

568. Hope BT, Michael GJ, Knigge KM, Vincent SR. Neuronal NADPH diaphorase is a nitric oxide synthase. Proc Natl Acad Sci USA 1991;88:2811-2814. [PubMed: 1707173]

569. Hotchkiss RS, Karl IE. The pathophysiology and treatment of sepsis. N Engl J Med 2003;348:138150. [PubMed: 12519925]

570. Hsu CM, Wang JS, Liu CH, Chen LW. Kupffer cells protect liver from ischemia-reperfusion injury by an inducible nitric oxide synthase-dependent mechanism. Shock 2002;17:280-285. [PubMed: 11954827]

571. Hsu HC, Zhou T, Kim H, Barnes S, Yang P, Wu Q, Zhou J, Freeman BA, Luo M, Mountz JD. Production of a novel class of polyreactive pathogenic autoantibodies in BXD2 mice causes glomerulonephritis and arthritis. Arthritis Rheum 2006;54:343-355. [PubMed: 16385526]

572. Hu P, Ischiropoulos H, Beckman JS, Matalon S. Peroxynitrite inhibition of oxygen consumption and sodium transport in alveolar type II cells. Am J Physiol Lung Cell Mol Physiol 1994;266:L628L634.

573. Huang CH, Vatner SF, Peppas AP, Yang G, Kudej RK. Cardiac nerves affect myocardial stunning through reactive oxygen and nitric oxide mechanisms. Circ Res 2003;93:866-873. [PubMed: 14512445]

574. Huang PL, Dawson TM, Bredt DS, Snyder SH, Fishman MC. Targeted disruption of the neuronal nitric oxide synthase gene. Cell 1993;75:1273-1286. [PubMed: 7505721] 
575. Huang Z, Huang PL, Ma J, Meng W, Ayata C, Fishman MC, Moskowitz MA. Enlarged infarcts in endothelial nitric oxide synthase knockout mice are attenuated by nitro-L-arginine. J Cereb Blood Flow Metab 1996;16:981-987. [PubMed: 8784243]

576. Huang Z, Huang PL, Panahian N, Dalkara T, Fishman MC, Moskowitz MA. Effects of cerebral ischemia in mice deficient in neuronal nitric oxide synthase. Science 1994;265:1883-1885. [PubMed: 7522345]

577. Hubbard SR, Till JH. Protein tyrosine kinase structure and function. Annu Rev Biochem 2000;69:373-398. [PubMed: 10966463]

578. Huber W, Menander-Huber KB, Saifer MG, Williams LD. Bioavailability of superoxide dismutase: implications for the anti-inflammatory action mechanism of orgotein. Agents Actions Suppl 1980;7:185-195. [PubMed: 6454334]

579. Hucks D, Thuraisingham RC, Raftery MJ, Yaqoob MM. Homocysteine induced impairment of nitric oxide-dependent vasorelaxation is reversible by the superoxide dismutase mimetic TEM-POL. Nephrol Dial Transplant 2004;19:1999-2005. [PubMed: 15252169]

580. Hughes MN, Nicklin HG. The chemistry of peroxonitrites. Part II. Copper (II)-catalysed reaction between hydroxylamine and peroxonitrite in alkali. J Chem Soc 1970;1970:925-928.

581. Hughes MN, Nicklin HG. A possible role for the species peroxonitrite in nitrification. Biochim Biophys Acta 1970;222:660-661. [PubMed: 5496491]

582. Huhmer AF, Nishida CR, Ortiz de Montellano PR, Schoneich C. Inactivation of the inducible nitric oxide synthase by peroxynitrite. Chem Res Toxicol 1997;10:618-626. [PubMed: 9168261]

583. Huie RE, Padmaja S. The reaction rate of nitric oxide with superoxide. Free Rad Res Commun 1993;18:195-199.

584. Hunter GC, Henderson AM, Westerband A, Kobayashi H, Suzuki F, Yan ZQ, Sirsjo A, Putnam $\mathrm{CW}$, Hansson GK. The contribution of inducible nitric oxide and cytomegalovirus to the stability of complex carotid plaque. J Vasc Surg 1999;30:36-50. [PubMed: 10394152]

585. Hussain SP, Trivers GE, Hofseth LJ, He P, Shaikh I, Mechanic LE, Doja S, Jiang W, Subleski J, Shorts L, Haines D, Laubach VE, Wiltrout RH, Djurickovic D, Harris CC. Nitric oxide, a mediator of inflammation, suppresses tumorigenesis. Cancer Res 2004;64:6849-6853. [PubMed: 15466171]

586. Hutchinson $F$. The distance that a radical formed by ionizing radiation can diffuse in a yeast cell. Radiat Res 1957;7:473-483. [PubMed: 13485388]

587. Iadecola C, Alexander M. Cerebral ischemia and inflammation. Curr Opin Neurol 2001;14:89-94. [PubMed: 11176223]

588. Iadecola C, Xu X, Zhang F, el-Fakahany EE, Ross ME. Marked induction of calcium-independent nitric oxide synthase activity after focal cerebral ischemia. J Cereb Blood Flow Metab 1995; 15:5259. [PubMed: 7528224]

589. Iadecola C, Zhang F, Casey R, Clark HB, Ross ME. Inducible nitric oxide synthase gene expression in vascular cells after transient focal cerebral ischemia. Stroke 1996;27:1373-1380. [PubMed: 8711805]

590. Iadecola C, Zhang F, Casey R, Nagayama M, Ross ME. Delayed reduction of ischemic brain injury and neurological deficits in mice lacking the inducible nitric oxide synthase gene. J Neurosci 1997;17:9157-9164. [PubMed: 9364062]

591. Iadecola C, Zhang F, Xu X. Inhibition of inducible nitric oxide synthase ameliorates cerebral ischemic damage. Am J Physiol Regul Integr Comp Physiol 1995;268:R286-R292.

592. Ialenti A, Ianaro A, Moncada S, Di Rosa M. Modulation of acute inflammation by endogenous nitric oxide. Eur J Pharmacol 1992;211:177-182. [PubMed: 1612108]

593. Ichinose F, Roberts JD Jr, Zapol WM. Inhaled nitric oxide: a selective pulmonary vasodilator: current uses and therapeutic potential. Circulation 2004;109:3106-3111. [PubMed: 15226227]

594. Ignarro LJ. Heme-dependent activation of soluble guanylate cyclase by nitric oxide: regulation of enzyme activity by porphyrins and metalloporphyrins. Semin Hematol 1989;26:63-76. [PubMed: 2564216]

595. Ignarro LJ. Nitric oxide: a unique endogenous signaling molecule in vascular biology. Biosci Rep 1999;19:51-71. [PubMed: 10888468] 
596. Ignarro LJ, Adams JB, Horwitz PM, Wood KS. Activation of soluble guanylate cyclase by NOhemoproteins involves NO-heme exchange. J Biol Chem 1986;261:4997-5002. [PubMed: 2870064]

597. Ignarro LJ, Harbison RG, Wood KS, Kadowitz PJ. Activation of purified soluble guanylate cyclase by endothelium-derived relaxing factor from intrapulmonary artery and vein: stimulation by acetylcholine, bradykinin and arachidonic acid. J Pharmacol Exp Ther 1986;237:893-900. [PubMed: 2872327]

598. Ilnytska O, Lyzogubov VV, Stevens MJ, Drel VR, Mashtalir N, Pacher P, Yorek MA, Obrosova IG. Poly(ADP-ribose) polymerase inhibition alleviates experimental diabetic sensory neuropathy. Diabetes 2006;55:1686-1694. [PubMed: 16731831]

599. Imai H, Graham DI, Masayasu H, Macrae IM. Antioxidant ebselen reduces oxidative damage in focal cerebral ischemia. Free Radic Biol Med 2003;34:56-63. [PubMed: 12498979]

600. Inauen W, Granger DN, Meininger CJ, Schelling ME, Granger HJ, Kvietys PR. Anoxiareoxygenation-induced, neutrophil-mediated endothelial cell injury: role of elastase. Am J Physiol Heart Circ Physiol 1990;259:H925-H931.

601. Inoue M, Inoue K. Role of free radicals in and around vascular endothelial cells in the mechanism of aging. Ann NY Acad Sci 1996;786:224-232. [PubMed: 8687022]

602. Inoue T, Kato T, Hikichi Y, Hashimoto S, Hirase T, Morooka T, Imoto Y, Takeda Y, Sendo F, Node K. Stent-induced neutrophil activation is associated with an oxidative burst in the inflammatory process, leading to neointimal thickening. Thromb Haemost 2006;95:43-48. [PubMed: 16543960]

603. Ip JH, Fuster V, Badimon L, Badimon J, Taubman MB, Chesebro JH. Syndromes of accelerated atherosclerosis: role of vascular injury and smooth muscle cell proliferation. J Am Coll Cardiol 1990;15:1667-1687. [PubMed: 2188991]

604. Iqbal M, Cohen RI, Marzouk K, Liu SF. Time course of nitric oxide, peroxynitrite, antioxidants in the endotoxemic heart. Crit Care Med 2002;30:1291-1296. [PubMed: 12072683]

605. Ischiropoulos H. Biological selectivity and functional aspects of protein tyrosine nitration. Biochem Biophys Res Commun 2003;305:776-783. [PubMed: 12763060]

606. Ischiropoulos H. Biological tyrosine nitration: a pathophysiological function of nitric oxide and reactive oxygen species. Arch Biochem Biophys 1998;356:1-11. [PubMed: 9681984]

607. Ischiropoulos H, al-Mehdi AB, Fisher AB. Reactive species in ischemic rat lung injury: contribution of peroxynitrite. Am J Physiol Lung Cell Mol Physiol 1995;269:L158-L164.

608. Ischiropoulos H, Beckman JS. Oxidative stress and nitration in neurodegeneration: cause, effect, or association? J Clin Invest 2003;111:163-169. [PubMed: 12531868]

609. Ischiropoulos H, Chen J, Tsai JHM, Martin JC, Smith CD, Beckman JS. Peroxynitrite (ONOO$\left.{ }^{-}\right)$ reacts with superoxide dismutase to give the reactive nitronium ion (Abstract). Free Rad Biol Med 1990;9:131.

610. Ischiropoulos H, Gow A, Thom SR, Kooy NW, Royall JA, Crow JP. Detection of reactive nitrogen species using 2,7-dichlorodihydrofluorescein and dihydrorhodamine 123. Methods Enzymol 1999;301:367-373. [PubMed: 9919585]

611. Ischiropoulos H, Zhu L, Beckman JS. Peroxynitrite formation from macrophage-derived nitric oxide. Arch Biochem Biophys 1992;298:446-451. [PubMed: 1329657]

612. Ischiropoulos H, Zhu L, Chen J, Tsai M, Martin JC, Smith CD, Beckman JS. Peroxynitrite-mediated tyrosine nitration catalyzed by superoxide dismutase. Arch Biochem Biophys 1992;298:431-437. [PubMed: 1416974]

613. Ishida H, Genka C, Nakazawa H. Application of authentic peroxynitrite to biological materials. Methods Enzymol 1999;301:402-409. [PubMed: 9919589]

614. Ishida H, Ichimori K, Hirota Y, Fukahori M, Nakazawa H. Peroxynitrite-induced cardiac myocyte injury. Free Radic Biol Med 1996;20:343-350. [PubMed: 8720904]

615. Ishii N, Patel KP, Lane PH, Taylor T, Bian K, Murad F, Pollock JS, Carmines PK. Nitric oxide synthesis and oxidative stress in the renal cortex of rats with diabetes mellitus. J Am Soc Nephrol 2001;12:1630-1639. [PubMed: 11461935]

616. Ishiyama S, Hiroe M, Nishikawa T, Abe S, Shimojo T, Ito H, Ozasa S, Yamakawa K, Matsuzaki M, Mohammed MU, Nakazawa H, Kasajima T, Marumo F. Nitric oxide contributes to the 
progression of myocardial damage in experimental autoimmune myocarditis in rats. Circulation 1997;95:489-496. [PubMed: 9008468]

617. Ito K, Hanazawa T, Tomita K, Barnes PJ, Adcock IM. Oxidative stress reduces histone deacetylase 2 activity and enhances IL- 8 gene expression: role of tyrosine nitration. Biochem Biophys Res Commun 2004;315:240-245. [PubMed: 15013452]

618. Jacobson GM, Dourron HM, Liu J, Carretero OA, Reddy DJ, Andrzejewski T, Pagano PJ. Novel $\mathrm{NAD}(\mathrm{P}) \mathrm{H}$ oxidase inhibitor suppresses angioplasty-induced superoxide and neointimal hyperplasia of rat carotid artery. Circ Res 2003;92:637-643. [PubMed: 12609967]

619. Jadeski LC, Chakraborty C, Lala PK. Nitric oxide-mediated promotion of mammary tumour cell migration requires sequential activation of nitric oxide synthase, guanylate cyclase and mitogenactivated protein kinase. Int J Cancer 2003;106:496-504. [PubMed: 12845643]

620. Jaeschke H. Glutathione disulfide formation and oxidant stress during acetaminophen-induced hepatotoxicity in mice in vivo: the protective effect of allopurinol. J Pharmacol Exp Ther 1990;255:935-941. [PubMed: 2262912]

621. Jaeschke H. Molecular mechanisms of hepatic ischemia-reperfusion injury and preconditioning. Am J Physiol Gastrointest Liver Physiol 2003;284:G15-G26. [PubMed: 12488232]

622. Jaeschke H, Bajt ML. Intracellular signaling mechanisms of acetaminophen-induced liver cell death. Toxicol Sci 2006;89:31-41. [PubMed: 16177235]

623. Jagtap P, Soriano FG, Virag L, Liaudet L, Mabley J, Szabo E, Hasko G, Marton A, Lorigados CB, Gallyas F Jr, Sumegi B, Hoyt DG, Baloglu E, VanDuzer J, Salzman AL, Southan GJ, Szabo C. Novel phenanthridinone inhibitors of poly (adenosine 5'-diphosphate-ribose) synthetase: potent cytoprotective and antishock agents. Crit Care Med 2002;30:1071-1082. [PubMed: 12006805]

624. Jagtap PG, Baloglu E, Southan GJ, Mabley JG, Li H, Zhou J, van Duzer J, Salzman AL, Szabo C. Discovery of potent poly-(ADP-ribose) polymerase-1 inhibitors from the modification of indeno [1,2-c]isoquinolinone. J Med Chem 2005;48:5100-5103. [PubMed: 16078828]

625. Jaiswal M, LaRusso NF, Nishioka N, Nakabeppu Y, Gores GJ. Human Ogg1, a protein involved in the repair of 8-oxoguanine, is inhibited by nitric oxide. Cancer Res 2001;61:6388-6393. [PubMed: $11522631]$

626. Jaiswal M, LaRusso NF, Shapiro RA, Billiar TR, Gores GJ. Nitric oxide-mediated inhibition of DNA repair potentiates oxidative DNA damage in cholangiocytes. Gastroenterology 2001;120:190-199. [PubMed: 11208728]

627. Jang B, Han S. Biochemical properties of cytochrome $c$ nitrated by peroxynitrite. Biochimie 2006;88:53-58. [PubMed: 16040185]

628. Janssen-Heininger YM, Persinger RL, Korn SH, Pantano C, McElhinney B, Reynaert NL, Langen RC, Ckless K, Shrivastava P, Poynter ME. Reactive nitrogen species and cell signaling: implications for death or survival of lung epithelium. Am J Respir Crit Care Med 2002;166:S9-S16. [PubMed: 12471083]

629. Javesghani D, Hussain SN, Scheidel J, Quinn MT, Magder SA. Superoxide production in the vasculature of lipopolysaccharide-treated rats and pigs. Shock 2003;19:486-493. [PubMed: 12744495]

630. Jay D, Hitomi H, Griendling KK. Oxidative stress and diabetic cardiovascular complications. Free Radic Biol Med 2006;40:183-192. [PubMed: 16413400]

631. Jenkins DC, Charles IG, Thomsen LL, Moss DW, Holmes LS, Baylis SA, Rhodes P, Westmore K, Emson PC, Moncada S. Roles of nitric oxide in tumor growth. Proc Natl Acad Sci USA 1995;92:4392-4396. [PubMed: 7538668]

632. Jenner P. Oxidative stress in Parkinson's disease. Ann Neurol 2003;53(Suppl 3):S26-S36. [PubMed: 12666096]

633. Ji Y, Neverova I, Van Eyk JE, Bennett BM. Nitration of tyrosine 92 mediates the activation of rat microsomal glutathione $S$-transferase by peroxynitrite. J Biol Chem 2006;281:1986-1991. [PubMed: 16314419]

634. Jijon HB, Churchill T, Malfair D, Wessler A, Jewell LD, Parsons HG, Madsen KL. Inhibition of poly(ADP-ribose) polymerase attenuates inflammation in a model of chronic colitis. Am J Physiol Gastrointest Liver Physiol 2000;279:G641-G651. [PubMed: 10960365] 
635. Jin L, Abou-Mohamed G, Caldwell RB, Caldwell RW. Endothelial cell dysfunction in a model of oxidative stress. Med Sci Monit 2001;7:585-591. [PubMed: 11433181]

636. Jones SP, Bolli R. The ubiquitous role of nitric oxide in cardioprotection. J Mol Cell Cardiol 2006;40:16-23. [PubMed: 16288777]

637. Jones SP, Greer JJ, Ware PD, Yang J, Walsh K, Lefer DJ. Deficiency of iNOS does not attenuate severe congestive heart failure in mice. Am J Physiol Heart Circ Physiol 2005;288:H365-H370. [PubMed: 15319210]

638. Jope RS, Zhang L, Song L. Peroxynitrite modulates the activation of p38 and extracellular regulated kinases in PC12 cells. Arch Biochem Biophys 2000;376:365-370. [PubMed: 10775424]

639. Joshi MS, Ferguson TB Jr, Han TH, Hyduke DR, Liao JC, Rassaf T, Bryan N, Feelisch M, Lancaster JR Jr. Nitric oxide is consumed, rather than conserved, by reaction with oxyhemoglobin under physiological conditions. Proc Natl Acad Sci USA 2002;99:10341-10346. [PubMed: 12124398]

640. Jozsef L, Khreiss T, El Kebir D, Filep JG. Activation of TLR-9 induces IL-8 secretion through peroxynitrite signaling in human neutrophils. J Immunol 2006;176:1195-1202. [PubMed: 16394009]

641. Jozsef L, Zouki C, Petasis NA, Serhan CN, Filep JG. Lipoxin A4 and aspirin-triggered 15-epi-lipoxin A4 inhibit peroxynitrite formation, NF-kappa B and AP-1 activation, and IL-8 gene expression in human leukocytes. Proc Natl Acad Sci USA 2002;99:13266-13271. [PubMed: 12235371]

642. Kacimi R, Karliner JS, Koudssi F, Long CS. Expression and regulation of adhesion molecules in cardiac cells by cytokines: response to acute hypoxia. Circ Res 1998;82:576-586. [PubMed: 9529162]

643. Kagota S, Yamaguchi Y, Tanaka N, Kubota Y, Kobayashi K, Nejime N, Nakamura K, Kunitomo M, Shinozuka K. Disturbances in nitric oxide/cyclic guanosine monophosphate system in SHR/ NDmcr-cp rats, a model of metabolic syndrome. Life Sci 2006;78:1187-1196. [PubMed: 16188278]

644. Kahl KG, Zielasek J, Uttenthal LO, Rodrigo J, Toyka KV, Schmidt HH. Protective role of the cytokine-inducible isoform of nitric oxide synthase induction and nitrosative stress in experimental autoimmune encephalomyelitis of the DA rat. J Neurosci Res 2003;73:198-205. [PubMed: 12836162]

645. Kaji T, Kaieda I, Hisatsune T, Kaminogawa S. 3-Morpholino-sydnonimine hydrochloride induces p53-dependent apoptosis in murine primary neural cells: a critical role for p21(ras)-MAPK-p19 (ARF) pathway. Nitric Oxide 2002;6:125-134. [PubMed: 11890736]

646. Kajstura J, Fiordaliso F, Andreoli AM, Li B, Chimenti S, Medow MS, Limana F, Nadal-Ginard B, Leri A, Anversa P. IGF-1 overexpression inhibits the development of diabetic cardiomyopathy and angiotensin II-mediated oxidative stress. Diabetes 2001;50:1414-1424. [PubMed: 11375343]

647. Kalb RG, Agostini J. Molecular evidence for nitric oxide-mediated motor neuron development. Neuroscience 1993;57:1-8. [PubMed: 7506398]

648. Kalia N, Pockley AG, Wood RF, Brown NJ. Effects of FK409 on intestinal ischemia-reperfusion injury and ischemia-induced changes in the rat mucosal villus microcirculation. Transplantation 2001;72:1875-1880. [PubMed: 11773883]

649. Kameoka M, Ota K, Tetsuka T, Tanaka Y, Itaya A, Okamoto T, Yoshihara K. Evidence for regulation of NF-kappaB by poly-(ADP-ribose) polymerase. Biochem J 2000;346:641-649. [PubMed: 10698690]

650. Kamisaki Y, Waldman SA, Murad F. The involvement of catalytic site thiol groups in the activation of soluble guanylate cyclase by sodium nitroprusside. Arch Biochem Biophys 1986;251:709-714. [PubMed: 2879512]

651. Kaneto H, Fujii J, Seo HG, Suzuki K, Matsuoka T, Nakamura M, Tatsumi H, Yamasaki Y, Kamada $\mathrm{T}$, Taniguchi N. Apoptotic cell death triggered by nitric oxide in pancreatic beta-cells. Diabetes 1995;44:733-738. [PubMed: 7540572]

652. Kang KW, Choi SH, Kim SG. Peroxynitrite activates NF-E2-related factor 2/antioxidant response element through the pathway of phosphatidylinositol 3-kinase: the role of nitric oxide synthase in rat glutathione $S$-transferase A2 induction. Nitric Oxide 2002;7:244-253. [PubMed: 12446173] 
653. Kano H, Hayashi T, Sumi D, Matusi-Hirai H, Tsunekawa T, Endo H, Iguchi A. Estriol retards and stabilizes atherosclerosis through an NO-mediated system. Life Sci 2002;71:31-42. [PubMed: 12020746]

654. Kanski J, Behring A, Pelling J, Schoneich C. Proteomic identification of 3-nitrotyrosine-containing rat cardiac proteins: effects of biological aging. Am J Physiol Heart Circ Physiol 2005;288:H371H381. [PubMed: 15345482]

655. Kanski J, Hong SJ, Schoneich C. Proteomic analysis of protein nitration in aging skeletal muscle and identification of nitrotyrosine-containing sequences in vivo by nanoelectrospray ionization tandem mass spectrometry. J Biol Chem 2005;280:24261-24266. [PubMed: 15851474]

656. Kase H, Hashikabe Y, Uchida K, Nakanishi N, Hattori Y. Supplementation with tetrahydrobiopterin prevents the cardiovascular effects of angiotensin II-induced oxidative and nitrosative stress. J Hypertens 2005;23:1375-1382. [PubMed: 15942460]

657. Kashiwagi S, Izumi Y, Gohongi T, Demou ZN, Xu L, Huang PL, Buerk DG, Munn LL, Jain RK, Fukumura D. NO mediates mural cell recruitment and vessel morphogenesis in murine melanomas and tissue-engineered blood vessels. J Clin Invest 2005;115:1816-1827. [PubMed: 15951843]

658. Kasina S, Rizwani W, Radhika KV, Singh SS. Nitration of profilin effects its interaction with poly (L-proline) and actin. J Biochem 2005;138:687-695. [PubMed: 16428297]

659. Kasina S, Wasia R, Fasim A, Radhika KV, Singh SS. Phorbol ester mediated activation of inducible nitric oxide synthase results in platelet profilin nitration. Nitric Oxide 2006;14:65-71. [PubMed: 16288977]

660. Kawachi S, Hines IN, Laroux FS, Hoffman J, Bharwani S, Gray L, Leffer D, Grisham MB. Nitric oxide synthase and post-ischemic liver injury. Biochem Biophys Res Commun 2000;276:851-854. [PubMed: 11027558]

661. Kawano T, Anrather J, Zhou P, Park L, Wang G, Frys KA, Kunz A, Cho S, Orio M, Iadecola C. Prostaglandin $\mathrm{E}_{2}$ EP1 receptors: downstream effectors of COX-2 neurotoxicity. Nat Med 2006;12:225-229. [PubMed: 16432513]

662. Keel M, Trentz O. Pathophysiology of polytrauma. Injury 2005;36:691-709. [PubMed: 15910820]

663. Keira N, Tatsumi T, Matoba S, Shiraishi J, Yamanaka S, Akashi K, Kobara M, Asayama J, Fushiki $\mathrm{S}$, Fliss H, Nakagawa M. Lethal effect of cytokine-induced nitric oxide and peroxynitrite on cultured rat cardiac myocytes. J Mol Cell Cardiol 2002;34:583-596. [PubMed: 12056861]

664. Keller JN, Kindy MS, Holtsberg FW, St Clair DK, Yen HC, Germeyer A, Steiner SM, Bruce-Keller AJ, Hutchins JB, Mattson MP. Mitochondrial manganese superoxide dismutase prevents neural apoptosis and reduces ischemic brain injury: suppression of peroxynitrite production, lipid peroxidation, mitochondrial dysfunction. J Neurosci 1998;18:687-697. [PubMed: 9425011]

665. Keller JN, Mattson MP. Roles of lipid peroxidation in modulation of cellular signaling pathways, cell dysfunction, death in the nervous system. Rev Neurosci 1998;9:105-116. [PubMed: 9711902]

666. Kennedy M, Denenberg AG, Szabo C, Salzman AL. Poly(ADP-ribose) synthetase activation mediates increased permeability induced by peroxynitrite in Caco-2BBe cells. Gastroenterology 1998;114:510-518. [PubMed: 9496941]

667. Kennedy S, Preston AA, McPhaden AR, Miller AM, Wainwright CL, Wadsworth RM. Correlation of changes in nitric oxide synthase, superoxide dismutase and nitrotyrosine with endothelial regeneration and neointimal hyperplasia in the balloon-injured rabbit subclavian artery. Coron Artery Dis 2004;15:337-346. [PubMed: 15346092]

668. Kettle AJ, van Dalen CJ, Winterbourn CC. Peroxynitrite and myeloperoxidase leave the same footprint in protein nitration. Redox Rep 1997;3:257-258. [PubMed: 9754322]

669. Keyer K, Imlay JA. Inactivation of dehydratase [4Fe-4S] clusters and disruption of iron homeostasis upon cell exposure to peroxynitrite. J Biol Chem 1997;272:27652-27659. [PubMed: 9346904]

670. Khadour FH, Panas D, Ferdinandy P, Schulze C, Csont T, Lalu MM, Wildhirt SM, Schulz R. Enhanced $\mathrm{NO}$ and superoxide generation in dysfunctional hearts from endotoxemic rats. Am $\mathrm{J}$ Physiol Heart Circ Physiol 2002;283:H1108-H1115. [PubMed: 12181141]

671. Khan F, Ali R. Antibodies against nitric oxide damaged poly L-tyrosine and 3-nitrotyrosine levels in systemic lupus erythematosus. J Biochem Mol Biol 2006;39:189-196. [PubMed: 16584635] 
672. Khan M, Jatana M, Elango C, Singh Paintlia A, Singh AK, Singh I. Cerebrovascular protection by various nitric oxide donors in rats after experimental stroke. Nitric Oxide 2006;15:114-124. [PubMed: 16524750]

673. Khor HK, Fisher MT, Schoneich C. Potential role of methionine sulfoxide in the inactivation of the chaperone GroEL by hypochlorous acid $(\mathrm{HOCl})$ and peroxynitrite $\left(\mathrm{ONOO}^{-}\right)$. J Biol Chem 2004;279:19486-19493. [PubMed: 14757771]

674. Khreiss T, Jozsef L, Potempa LA, Filep JG. Loss of pentameric symmetry in C-reactive protein induces interleukin-8 secretion through peroxynitrite signaling in human neutrophils. Circ Res 2005;97:690-697. [PubMed: 16123332]

675. Killestein J, Polman CH. Current trials in multiple sclerosis: established evidence and future hopes. Curr Opin Neurol 2005;18:253-260. [PubMed: 15891408]

676. Kim CH, Zou Y, Kim DH, Kim ND, Yu BP, Chung HY. Proteomic analysis of nitrated and 4hydroxy-2-nonenal-modified serum proteins during aging. J Gerontol A Biol Sci Med Sci 2006;61:332-338. [PubMed: 16611698]

677. Kim GW, Kondo T, Noshita N, Chan PH. Manganese superoxide dismutase deficiency exacerbates cerebral infarction after focal cerebral ischemia/reperfusion in mice: implications for the production and role of superoxide radicals. Stroke 2002;33:809-815. [PubMed: 11872908]

678. King PA, Anderson VE, Edwards JO, Gustafson G, Plumb RC, Suggs JW. A stable solid that generates hydroxyl radical upon dissolution in aqueous solutions: reaction with proteins and nucleic acid. J Am Chem Soc 1992;114:5430-5432.

679. Kinobe R, Ji Y, Nakatsu K. Peroxynitrite-mediated inactivation of heme oxygenases. BMC Pharmacol 2004;4:26. [PubMed: 15498099]

680. Kinsella JP. Inhaled nitric oxide therapy in premature newborns. Curr Opin Pediatr 2006;18:107111. [PubMed: 16601487]

681. Kinsella JP, Cutter GR, Walsh WF, Gerstmann DR, Bose CL, Hart C, Sekar KC, Auten RL, Bhutani VK, Gerdes JS, George TN, Southgate WM, Carriedo H, Couser RJ, Mammel MC, Hall DC, Pappagallo M, Sardesai S, Strain JD, Baier M, Abman SH. Early inhaled nitric oxide therapy in premature newborns with respiratory failure. N Engl J Med 2006;355:354-364. [PubMed: 16870914]

682. Kisley LR, Barrett BS, Bauer AK, Dwyer-Nield LD, Barthel B, Meyer AM, Thompson DC, Malkinson AM. Genetic ablation of inducible nitric oxide synthase decreases mouse lung tumorigenesis. Cancer Res 2002;62:6850-6856. [PubMed: 12460898]

683. Kiss J, Lamarque D, Delchier JC, Whittle BJ. Time-dependent actions of nitric oxide synthase inhibition on colonic inflammation induced by trinitrobenzene sulphonic acid in rats. Eur $\mathrm{J}$ Pharmacol 1997;336:219-224. [PubMed: 9384236]

684. Kiziltepe T, Yan A, Dong M, Jonnalagadda VS, Dedon PC, Engelward BP. Delineation of the chemical pathways underlying nitric oxide-induced homologous recombination in mammalian cells. Chem Biol 2005;12:357-369. [PubMed: 15797220]

685. Kleemann R, Rothe H, Kolb-Bachofen V, Xie QW, Nathan C, Martin S, Kolb H. Transcription and translation of inducible nitric oxide synthase in the pancreas of prediabetic BB rats. FEBS Lett 1993;328:9-12. [PubMed: 7688327]

686. Klein JA, Ackerman SL. Oxidative stress, cell cycle, neurodegeneration. J Clin Invest 2003;111:785-793. [PubMed: 12639981]

687. Klotz LO, Schieke SM, Sies H, Holbrook NJ. Peroxynitrite activates the phosphoinositide 3-kinase/ Akt pathway in human skin primary fibroblasts. Biochem J 2000;352:219-225. [PubMed: 11062076]

688. Klug D, Rabani J, Fridovich I. A direct demonstration of the catalytic action of superoxide dismutase through the use of pulse radiolysis. J Biol Chem 1972;247:4839-4842. [PubMed: 4626367]

689. Knapp LT, Kanterewicz BI, Hayes EL, Klann E. Peroxynitrite-induced tyrosine nitration and inhibition of protein kinase C. Biochem Biophys Res Commun 2001;286:764-770. [PubMed: 11520063]

690. Knight TR, Kurtz A, Bajt ML, Hinson JA, Jaeschke H. Vascular and hepatocellular peroxynitrite formation during acetaminophen toxicity: role of mitochondrial oxidant stress. Toxicol Sci 2001;62:212-220. [PubMed: 11452133] 
691. Knight-Lozano CA, Young CG, Burow DL, Hu ZY, Uyeminami D, Pinkerton KE, Ischiropoulos $\mathrm{H}$, Ballinger SW. Cigarette smoke exposure and hypercholesterolemia increase mitochondrial damage in cardiovascular tissues. Circulation 2002;105:849-854. [PubMed: 11854126]

692. Knyushko TV, Sharov VS, Williams TD, Schoneich C, Bigelow DJ. 3-Nitrotyrosine modification of SERCA2a in the aging heart: a distinct signature of the cellular redox environment. Biochemistry 2005;44:13071-13081. [PubMed: 16185075]

693. Kobayashi N, Horinaka S, Mita S, Yoshida K, Honda T, Kobayashi T, Hara K, Nishikimi T, Matsuoka H. Aminoguanidine inhibits mitogen-activated protein kinase and improves cardiac performance and cardiovascular remodeling in failing hearts of salt-sensitive hypertensive rats. $\mathbf{J}$ Hypertens 2002;20:2475-2485. [PubMed: 12473873]

694. Koch M, De Keyser J. Uric acid in multiple sclerosis. Neurol Res 2006;28:316-319. [PubMed: 16687059]

695. Koeck T, Levison B, Hazen SL, Crabb JW, Stuehr DJ, Aulak KS. Tyrosine nitration impairs mammalian aldolase A activity. Mol Cell Proteomics 2004;3:548-557. [PubMed: 14978198]

696. Koh DW, Dawson TM, Dawson VL. Mediation of cell death by poly(ADP-ribose) polymerase-1. Pharmacol Res 2005;52:5-14. [PubMed: 15911329]

697. Koh SH, Park Y, Song CW, Kim JG, Kim K, Kim J, Kim MH, Lee SR, Kim DW, Yu HJ, Chang DI, Hwang SJ, Kim SH. The effect of PARP inhibitor on ischaemic cell death, its related inflammation and survival signals. Eur J Neurosci 2004;20:1461-1472. [PubMed: 15355313]

698. Koller A. Signaling pathways of mechanotransduction in arteriolar endothelium and smooth muscle cells in hypertension. Microcirculation 2002;9:277-294. [PubMed: 12152104]

699. Koller A, Huang A. Impaired nitric oxide-mediated flow-induced dilation in arterioles of spontaneously hypertensive rats. Circ Res 1994;74:416-421. [PubMed: 8118950]

700. Koller WC. Paraquat and Parkinson's disease. Neurology 1986;36:1147. [PubMed: 3736891]

701. Kondo T, Reaume AG, Huang TT, Carlson E, Murakami K, Chen SF, Hoffman EK, Scott RW, Epstein CJ, Chan PH. Reduction of CuZn-superoxide dismutase activity exacerbates neuronal cell injury and edema formation after transient focal cerebral ischemia. J Neurosci 1997;17:4180-4189. [PubMed: 9151735]

702. Kong SK, Yim MB, Stadtman ER, Chock PB. Peroxynitrite disables the tyrosine phosphorylation regulatory mechanism: lymphocyte-specific tyrosine kinase fails to phosphorylate nitrated cdc2(620)NH2 peptide. Proc Natl Acad Sci USA 1996;93:3377-3382. [PubMed: 8622943]

703. Konorev EA, Hogg N, Kalyanaraman B. Rapid and irreversible inhibition of creatine kinase by peroxynitrite. FEBS Lett 1998;427:171-174. [PubMed: 9607305]

704. Kooy NW, Lewis SJ. Elevation in arterial blood pressure following the development of tachyphylaxis to peroxynitrite. Eur J Pharmacol 1996;307:R5-R7. [PubMed: 8836625]

705. Kooy NW, Lewis SJ. Nitrotyrosine attenuates the hemodynamic effects of adrenoceptor agonists in vivo: relevance to the pathophysiology of peroxynitrite. Eur J Pharmacol 1996;310:155-161. [PubMed: 8884212]

706. Kooy NW, Lewis SJ. The peroxynitrite product 3-nitro-L-tyrosine attenuates the hemodynamic responses to angiotensin II in vivo. Eur J Pharmacol 1996;315:165-170. [PubMed: 8960880]

707. Kooy NW, Lewis SJ, Royall JA, Ye YZ, Kelly DR, Beckman JS. Extensive tyrosine nitration in human myocardial inflammation: evidence for the presence of peroxynitrite. Crit Care Med 1997;25:812-819. [PubMed: 9187601]

708. Koppenol WH. 100 years of peroxynitrite chemistry and 11 years of peroxynitrite biochemistry. Redox Rep 2001;6:339-341. [PubMed: 11865973]

709. Koppenol WH, Moreno JJ, Pryor WA, Ischiropoulos H, Beckman JS. Peroxynitrite, a cloaked oxidant formed by nitric oxide and superoxide. Chem Res Toxicol 1992;5:834-842. [PubMed: 1336991]

710. Koprowski H, Spitsin SV, Hooper DC. Prospects for the treatment of multiple sclerosis by raising serum levels of uric acid, a scavenger of peroxynitrite. Ann Neurol 2001;49:139. [PubMed: 11198290]

711. Kossenjans W, Eis A, Sahay R, Brockman D, Myatt L. Role of peroxynitrite in altered fetal-placental vascular reactivity in diabetes or preeclampsia. Am J Physiol Heart Circ Physiol 2000;278:H1311H1319. [PubMed: 10749729] 
712. Kowluru RA. Effect of reinstitution of good glycemic control on retinal oxidative stress and nitrative stress in diabetic rats. Diabetes 2003;52:818-823. [PubMed: 12606525]

713. Kowluru RA, Koppolu P, Chakrabarti S, Chen S. Diabetes-induced activation of nuclear transcriptional factor in the retina, its inhibition by antioxidants. Free Radic Res 2003;37:11691180. [PubMed: 14703729]

714. Krieglstein CF, Cerwinka WH, Laroux FS, Salter JW, Russell JM, Schuermann G, Grisham MB, Ross CR, Granger DN. Regulation of murine intestinal inflammation by reactive metabolites of oxygen and nitrogen: divergent roles of superoxide and nitric oxide. J Exp Med 2001;194:12071218. [PubMed: 11696587]

715. Kroncke KD, Funda J, Berschick B, Kolb H, Kolb-Bachofen V. Macrophage cytotoxicity towards isolated rat islet cells: neither lysis nor its protection by nicotinamide are beta-cell specific. Diabetologia 1991;34:232-238. [PubMed: 1829693]

716. Krupinski J, Vodovotz Y, Li C, Slowik A, Beevers D, Flanders KC, Lip G, Kumar P, Szczudlik A. Inducible nitric oxide production and expression of transforming growth factor-beta1 in serum and CSF after cerebral ischaemic stroke in man. Nitric Oxide 1998;2:442-453. [PubMed: 10342487]

717. Kubes P. Ischemia-reperfusion in feline small intestine: a role for nitric oxide. Am J Physiol Gastrointest Liver Physiol 1993;264:G143-G149.

718. Kuhn DM, Aretha CW, Geddes TJ. Peroxynitrite inactivation of tyrosine hydroxylase: mediation by sulfhydryl oxidation, not tyrosine nitration. J Neurosci 1999;19:10289-10294. [PubMed: 10575026]

719. Kuhn DM, Geddes TJ. Peroxynitrite inactivates tryptophan hydroxylase via sulfhydryl oxidation. Coincident nitration of enzyme tyrosyl residues has minimal impact on catalytic activity. J Biol Chem 1999;274:29726-29732. [PubMed: 10514446]

720. Kuhn DM, Geddes TJ. Reduced nicotinamide nucleotides prevent nitration of tyrosine hydroxylase by peroxynitrite. Brain Res 2002;933:85-89. [PubMed: 11929639]

721. Kuhn DM, Sadidi M, Liu X, Kreipke C, Geddes T, Borges C, Watson JT. Peroxynitrite-induced nitration of tyrosine hydroxylase: identification of tyrosines $423,428,432$ as sites of modification by matrix-assisted laser desorption ionization time-of-flight mass spectrometry and tyrosinescanning mutagenesis. J Biol Chem 2002;277:14336-14342. [PubMed: 11834745]

722. Kuroki I, Miyazaki T, Mizukami I, Matsumoto N, Matsumoto I. Effect of sodium nitroprusside on ischemia-reperfusion injuries of the rat liver. Hepatogastroenterology 2004;51:1404-1407. [PubMed: 15362764]

723. Kurzelewski M, Czarnowska E, Beresewicz A. Superoxide-and nitric oxide-derived species mediate endothelial dysfunction, endothelial glycocalyx disruption, enhanced neutrophil adhesion in the post-ischemic guinea-pig heart. J Physiol Pharmacol 2005;56:163-178. [PubMed: 15985700]

724. Kuster GM, Kotlyar E, Rude MK, Siwik DA, Liao R, Colucci WS, Sam F. Mineralocorticoid receptor inhibition ameliorates the transition to myocardial failure and decreases oxidative stress and inflammation in mice with chronic pressure overload. Circulation 2005;111:420-427. [PubMed: 15687129]

725. Kuzkaya N, Weissmann N, Harrison DG, Dikalov S. Interactions of peroxynitrite, tetrahydrobiopterin, ascorbic acid, thiols: implications for uncoupling endothelial nitric-oxide synthase. J Biol Chem 2003;278:22546-22554. [PubMed: 12692136]

726. Lakatta EG. Arterial and cardiac aging: major shareholders in cardiovascular disease enterprises. Part III: cellular and molecular clues to heart and arterial aging. Circulation 2003;107:490-497. [PubMed: 12551876]

727. Lakatta EG, Levy D. Arterial and cardiac aging: major shareholders in cardiovascular disease enterprises. Part II: the aging heart in health: links to heart disease. Circulation 2003;107:346-354. [PubMed: 12538439]

728. Lancaster JR. Simulation of the diffusion and reaction of endogenously produced nitric oxide. Proc Natl Acad Sci USA 1994;91:8137-8141. [PubMed: 8058769]

729. Lancel S, Tissier S, Mordon S, Marechal X, Depontieu F, Scherpereel A, Chopin C, Neviere R. Peroxynitrite decomposition catalysts prevent myocardial dysfunction and inflammation in endotoxemic rats. J Am Coll Cardiol 2004;43:2348-2358. [PubMed: 15193704] 
730. Landino LM, Iwig JS, Kennett KL, Moynihan KL. Repair of peroxynitrite damage to tubulin by the thioredoxin reductase system. Free Radic Biol Med 2004;36:497-506. [PubMed: 14975452]

731. Landmesser U, Drexler H. The clinical significance of endothelial dysfunction. Curr Opin Cardiol 2005;20:547-551. [PubMed: 16234629]

732. Lanone S, Manivet P, Callebert J, Launay JM, Payen D, Aubier M, Boczkowski J, Mebazaa A. Inducible nitric oxide synthase (NOS2) expressed in septic patients is nitrated on selected tyrosine residues: implications for enzymic activity. Biochem J 2002;366:399-404. [PubMed: 12097137]

733. Lassegue B, Sorescu D, Szocs K, Yin Q, Akers M, Zhang Y, Grant SL, Lambeth JD, Griendling KK. Novel gp91(phox) homologues in vascular smooth muscle cells: nox1 mediates angiotensin II-induced superoxide formation and redox-sensitive signaling pathways. Circ Res 2001;88:888894. [PubMed: 11348997]

734. Laursen JB, Somers M, Kurz S, McCann L, Warnholtz A, Freeman BA, Tarpey M, Fukai T, Harrison DG. Endothelial regulation of vasomotion in apoE-deficient mice: implications for interactions between peroxynitrite and tetrahydrobiopterin. Circulation 2001;103:1282-1288. [PubMed: 11238274]

735. Lee C, Miura K, Liu X, Zweier JL. Biphasic regulation of leukocyte superoxide generation by nitric oxide and peroxynitrite. J Biol Chem 2000;275:38965-38972. [PubMed: 10976106]

736. Lee CI, Liu X, Zweier JL. Regulation of xanthine oxidase by nitric oxide and peroxynitrite. J Biol Chem 2000;275:9369-9376. [PubMed: 10734080]

737. Lee CK, Allison DB, Brand J, Weindruch R, Prolla TA. Transcriptional profiles associated with aging and middle age-onset caloric restriction in mouse hearts. Proc Natl Acad Sci USA 2002;99:14988-14993. [PubMed: 12419851]

738. Lee JH, Yang ES, Park JW. Inactivation of $\mathrm{NADP}^{+}$-dependent isocitrate dehydrogenase by peroxynitrite. Implications for cytotoxicity and alcohol-induced liver injury. J Biol Chem 2003;278:51360-51371. [PubMed: 14551203]

739. Lee VG, Johnson ML, Baust J, Laubach VE, Watkins SC, Billiar TR. The roles of iNOS in liver ischemia-reperfusion injury. Shock 2001;16:355-360. [PubMed: 11699073]

740. Lee WH, Gounarides JS, Roos ES, Wolin MS. Influence of peroxynitrite on energy metabolism and cardiac function in a rat ischemia-reperfusion model. Am J Physiol Heart Circ Physiol 2003;285:H1385-H1395. [PubMed: 12816754]

741. Lees KR, Zivin JA, Ashwood T, Davalos A, Davis SM, Diener HC, Grotta J, Lyden P, Shuaib A, Hardemark HG, Wasiewski WW. NXY-059 for acute ischemic stroke. N Engl J Med 2006;354:588600. [PubMed: 16467546]

742. Leeuwenburgh C, Hardy MM, Hazen SL, Wagner P, Ohishi S, Steinbrecher UP, Heinecke JW. Reactive nitrogen intermediates promote low density lipoprotein oxidation in human atherosclerotic intima. J Biol Chem 1997;272:1433-1436. [PubMed: 8999808]

743. Lefer AM, Lefer DJ. The role of nitric oxide and cell adhesion molecules on the microcirculation in ischaemia-reperfusion. Cardiovasc Res 1996;32:743-751. [PubMed: 8915192]

744. Lefer DJ, Scalia R, Campbell B, Nossuli T, Hayward R, Salamon M, Grayson J, Lefer AM. Peroxynitrite inhibits leukocyte-endothelial cell interactions and protects against ischemiareperfusion injury in rats. J Clin Invest 1997;99:684-691. [PubMed: 9045871]

745. Lehnert M, Arteel GE, Smutney OM, Conzelmann LO, Zhong Z, Thurman RG, Lemasters JJ. Dependence of liver injury after hemorrhage/resuscitation in mice on NADPH oxidase-derived superoxide. Shock 2003;19:345-351. [PubMed: 12688546]

746. Lei SZ, Pan ZH, Aggarwal SK, Chen HS, Hartman J, Sucher NJ, Lipton SA. Effect of nitric oxide production on the redox modulatory site of the NMDA receptor-channel complex. Neuron 1992;8:1087-1099. [PubMed: 1376999]

747. Leite PF, Danilovic A, Moriel P, Dantas K, Marklund S, Dantas AP, Laurindo FR. Sustained decrease in superoxide dismutase activity underlies constrictive remodeling after balloon injury in rabbits. Arterioscler Thromb Vasc Biol 2003;23:2197-2202. [PubMed: 12958042]

748. Lepoivre M, Houee-Levin C, Coeytaux K, Decottignies P, Auger G, Lemaire G. Nitration of the tyrosyl radical in ribonucleotide reductase by nitrogen dioxide: a gamma radiolysis study. Free Radic Biol Med 2005;38:1511-1517. [PubMed: 15890625] 
749. Levrand S, Pesse B, Feihl F, Waeber B, Pacher P, Rolli J, Schaller MD, Liaudet L. Peroxynitrite is a potent inhibitor of NF-kappa B activation triggered by inflammatory stimuli in cardiac and endothelial cell lines. J Biol Chem 2005;280:34878-34887. [PubMed: 16079150]

750. Levrand S, Vannay-Bouchiche C, Pesse B, Pacher P, Feihl F, Waeber B, Liaudet L. Peroxynitrite is a major trigger of cardiomyocyte apoptosis in vitro and in vivo. Free Radic Biol Med 2006;41:886-895. [PubMed: 16934671]

751. Lewis SJ, Hoque A, Sandock K, Robertson TP, Bates JN, Kooy NW. Differential effects of peroxynitrite on the function of arginine vasopressin $\mathrm{V}(1 \mathrm{a})$ receptors and alpha(1)-adrenoceptors in vivo. Vasc Pharmacol. In press

752. Lewis SJ, Hoque A, Walton TM, Kooy NW. Potential role of nitration and oxidation reactions in the effects of peroxynitrite on the function of beta-adrenoceptor sub-types in the rat. Eur J Pharmacol 2005;518:187-194. [PubMed: 16043170]

753. Li F, Szabo C, Pacher P, Southan GJ, Abatan OI, Char-niauskaya T, Stevens MJ, Obrosova IG. Evaluation of orally active poly(ADP-ribose) polymerase inhibitor in streptozotocin-diabetic rat model of early peripheral neuropathy. Diabetologia 2004;47:710-717. [PubMed: 15298348]

754. Li H, Gutterman DD, Rusch NJ, Bubolz A, Liu Y. Nitration and functional loss of voltage-gated K ${ }^{+}$channels in rat coronary microvessels exposed to high glucose. Diabetes 2004;53:2436-2442. [PubMed: 15331556]

755. Li J, Li W, Su J, Liu W, Altura BT, Altura BM. Peroxynitrite induces apoptosis in rat aortic smooth muscle cells: possible relation to vascular diseases. Exp Biol Med 2004;229:264-269.

756. Li J, Su J, Li W, Liu W, Altura BT, Altura BM. Peroxynitrite induces apoptosis in canine cerebral vascular muscle cells: possible relation to neurodegenerative diseases and strokes. Neurosci Lett 2003;350:173-177. [PubMed: 14550922]

757. Li JM, Shah AM. Endothelial cell superoxide generation: regulation and relevance for cardiovascular pathophysiology. Am J Physiol Regul Integr Comp Physiol 2004;287:R1014-R1030. [PubMed: 15475499]

758. Li MH, Cha YN, Surh YJ. Carbon monoxide protects PC12 cells from peroxynitrite-induced apoptotic death by preventing the depolarization of mitochondrial transmembrane potential. Biochem Biophys Res Commun 2006;342:984-990. [PubMed: 16598857]

759. Li N, Karin M. Is NF-kappaB the sensor of oxidative stress? FASEB J 1999;13:1137-1143. [PubMed: 10385605]

760. Li X, De Sarno P, Song L, Beckman JS, Jope RS. Peroxynitrite modulates tyrosine phosphorylation and phosphoinositide signalling in human neuroblastoma SH-SY5Y cells: attenuated effects in human 1321N1 astrocytoma cells. Biochem J 1998;331:599-606. [PubMed: 9531503]

761. Li Y, Huang TT, Carlson EJ, Melov S, Ursell PC, Olson JL, Noble LJ, Yoshimura MP, Berger C, Chan PH, Wallace DC, Epstein CJ. Dilated cardiomyopathy and neonatal lethality in mutant mice lacking manganese superoxide dismutase. Nature Genet 1995;11:376-381. [PubMed: 7493016]

762. Liaudet L. Poly(adenosine 5'-diphosphate) ribose polymerase activation as a cause of metabolic dysfunction in critical illness. Curr Opin Clin Nutr Metab Care 2002;5:175-184. [PubMed: 11844985]

763. Liaudet L, Mabley JG, Soriano FG, Pacher P, Marton A, Hasko G, Szabo C. Inosine reduces systemic inflammation and improves survival in septic shock induced by cecal ligation and puncture. Am J Respir Crit Care Med 2001;164:1213-1220. [PubMed: 11673212]

764. Liaudet L, Murthy KG, Mabley JG, Pacher P, Soriano FG, Salzman AL, Szabo C. Comparison of inflammation, organ damage, oxidant stress induced by Salmonella enterica serovar Muenchen flagellin and serovar Enteritidis lipopolysaccharide. Infect Immun 2002;70:192-198. [PubMed: 11748182]

765. Liaudet L, Oddo M. Role of poly(adenosine diphosphate-ribose) polymerase 1 in septic peritonitis. Curr Opin Crit Care 2003;9:152-158. [PubMed: 12657979]

766. Liaudet L, Soriano FG, Szabo C. Biology of nitric oxide signaling. Crit Care Med 2000;28:N3752. [PubMed: 10807315]

767. Liaudet L, Soriano FG, Szabo E, Virag L, Mabley JG, Salz-man AL, Szabo C. Protection against hemorrhagic shock in mice genetically deficient in poly(ADP-ribose)polymerase. Proc Natl Acad Sci USA 2000;97:10203-10208. [PubMed: 10954738] 
768. Liaudet L, Szabo A, Soriano FG, Zingarelli B, Szabo C, Salzman AL. Poly(ADP-ribose) synthetase mediates intestinal mucosal barrier dysfunction after mesenteric ischemia. Shock 2000;14:134-141. [PubMed: 10947156]

769. Liaudet L, Szabo G, Szabo C. Oxidative stress and regional ischemia-reperfusion injury: the peroxynitrite-poly(ADP-ribose) polymerase connection. Coron Artery Dis 2003;14:115-122. [PubMed: 12655275]

770. Liaudet L, Yang Z, Al-Affar EB, Szabo C. Myocardial ischemic preconditioning in rodents is dependent on poly (ADP-ribose) synthetase. Mol Med 2001;7:406-417. [PubMed: 11474134]

771. Libermann TA, Baltimore D. Activation of interleukin-6 gene expression through the NF-kappa B transcription factor. Mol Cell Biol 1990;10:2327-2334. [PubMed: 2183031]

772. Lin HL, Kent UM, Zhang H, Waskell L, Hollenberg PF. Mutation of tyrosine 190 to alanine eliminates the inactivation of cytochrome $\mathrm{P} 450$ 2B1 by peroxynitrite. Chem Res Toxicol 2003;16:129-136. [PubMed: 12588183]

773. Lin KT, Xue JY, Nomen M, Spur B, Wong PY. Peroxynitrite-induced apoptosis in HL-60 cells. J Biol Chem 1995;270:16487-16490. [PubMed: 7622447]

774. Ling H, Gengaro PE, Edelstein CL, Martin PY, Wangsiripaisan A, Nemenoff R, Schrier RW. Effect of hypoxia on proximal tubules isolated from nitric oxide synthase knockout mice. Kidney Int 1998;53:1642-1646. [PubMed: 9607195]

775. Linke A, Recchia F, Zhang X, Hintze TH. Acute and chronic endothelial dysfunction: implications for the development of heart failure. Heart Fail Rev 2003;8:87-97. [PubMed: 12652162]

776. Linz W, Itter G, Dobrucki LW, Malinski T, Wiemer G. Ramipril improves nitric oxide availability in hypertensive rats with failing hearts after myocardial infarction. J Renin Angiotensin Aldosterone Syst 2003;4:180-185. [PubMed: 14608524]

777. Liochev SI, Fridovich I. Reversal of the superoxide dismutase reaction revisited. Free Radic Biol Med 2003;34:908-910. [PubMed: 12654479]

778. Lipton P. Ischemic cell death in brain neurons. Physiol Rev 1999;79:1431-1568. [PubMed: 10508238]

779. Lipton SA, Choi YB, Pan ZH, Lei SZ, Chen HS, Sucher NJ, Loscalzo J, Singel DJ, Stamler JS. A redox-based mechanism for the neuroprotective and neurodestructive effects of nitric oxide and related nitroso-compounds. Nature 1993;364:626-632. [PubMed: 8394509]

780. Liu JS, Zhao ML, Brosnan CF, Lee SC. Expression of inducible nitric oxide synthase and nitrotyrosine in multiple sclerosis lesions. Am J Pathol 2001;158:2057-2066. [PubMed: 11395383]

781. Liu P, Hock CE, Nagele R, Wong PY. Formation of nitric oxide, superoxide, peroxynitrite in myocardial ischemia-reperfusion injury in rats. Am J Physiol Heart Circ Physiol 1997;272:H2327$\mathrm{H} 2336$.

782. Liu P, Xu B, Quilley J, Wong PY. Peroxynitrite attenuates hepatic ischemia-reperfusion injury. Am J Physiol Cell Physiol 2000;279:C1970-C1977. [PubMed: 11078713]

783. Liu S, Beckman JS, Ku DD. Peroxynitrite, a product of superoxide and nitric oxide, produces coronary vasorelaxation in dogs. J Pharmacol Exp Ther 1994;268:1114-1121. [PubMed: 8138924]

784. Liu X, Miller MJ, Joshi MS, Thomas DD, Lancaster JR Jr. Accelerated reaction of nitric oxide with $\mathrm{O}_{2}$ within the hydrophobic interior of biological membranes. Proc Natl Acad Sci USA 1998;95:2175-2179. [PubMed: 9482858]

785. Liu Y, Gutterman DD. Oxidative stress and potassium channel function. Clin Exp Pharmacol Physiol 2002;29:305-311. [PubMed: 11985541]

786. Liu Y, Terata K, Chai Q, Li H, Kleinman LH, Gutterman DD. Peroxynitrite inhibits $\mathrm{Ca}^{2+}$-activated $\mathrm{K}^{+}$channel activity in smooth muscle of human coronary arterioles. Circ Res 2002;91:1070-1076. [PubMed: 12456494]

787. Lo CC, Chen JC, Chen HM, Shyr MH, Lau YT, Lin JN, Chen MF. Aminoguanidine attenuates hemodynamic and microcirculatory derangement in rat intestinal ischemia and reperfusion. $\mathrm{J}$ Trauma 1999;47:1108-1113. [PubMed: 10608542]

788. Loehe F, Preissler G, Annecke T, Bittmann I, Jauch KW, Messmer K. Continuous infusion of nitroglycerin improves pulmonary graft function of non-heart-beating donor lungs. Transplantation 2004;77:1803-1808. [PubMed: 15223895] 
789. Lokuta AJ, Maertz NA, Meethal SV, Potter KT, Kamp TJ, Valdivia HH, Haworth RA. Increased nitration of sarcoplasmic reticulum $\mathrm{Ca}^{2+}$-ATPase in human heart failure. Circulation 2005;111:988-995. [PubMed: 15710754]

790. Lopez BL, Liu GL, Christopher TA, Ma XL. Peroxynitrite, the product of nitric oxide and superoxide, causes myocardial injury in the isolated perfused rat heart. Coron Artery Dis 1997;8:149-153. [PubMed: 9237024]

791. Lopez CJ, Qayyum I, Mishra OP, Delivoria-Papadopoulos M. Effect of nitration on protein tyrosine phosphatase and protein phosphatase activity in neuronal cell membranes of newborn piglets. Neurosci Lett 2005;386:78-81. [PubMed: 16039061]

792. Los M, Mozoluk M, Ferrari D, Stepczynska A, Stroh C, Renz A, Herceg Z, Wang ZQ, SchulzeOsthoff K. Activation and caspase-mediated inhibition of PARP: a molecular switch between fibroblast necrosis and apoptosis in death receptor signaling. Mol Biol Cell 2002;13:978-988. [PubMed: 11907276]

793. Love S, Barber R, Wilcock GK. Neuronal accumulation of poly-(ADP-ribose) after brain ischaemia. Neuropathol Appl Neurobiol 1999;25:98-103. [PubMed: 10215997]

794. Love S, Barber R, Wilcock GK. Neuronal death in brain infarcts in man. Neuropathol Appl Neurobiol 2000;26:55-66. [PubMed: 10736067]

795. Low SY, Sabetkar M, Bruckdorfer KR, Naseem KM. The role of protein nitration in the inhibition of platelet activation by peroxynitrite. FEBS Lett 2002;511:59-64. [PubMed: 11821049]

796. Lowell CA. Src-family kinases: rheostats of immune cell signaling. Mol Immunol 2004;41:631643. [PubMed: 15220000]

797. Lowenstein CJ, Hill SL, Lafond-Walker A, Wu J, Allen G, Landavere M, Rose NR, Herskowitz A. Nitric oxide inhibits viral replication in murine myocarditis. J Clin Invest 1996;97:1837-1843. [PubMed: 8621766]

798. Lu Y, Wang X, Cederbaum AI. Lipopolysaccharide-induced liver injury in rats treated with the CYP2E1 inducer pyrazole. Am J Physiol Gastrointest Liver Physiol 2005;289:G308-G319. [PubMed: 15845871]

799. Lucchesi BR. Modulation of leukocyte-mediated myocardial reperfusion injury. Annu Rev Physiol 1990;52:561-576. [PubMed: 2184767]

800. Lucchesi BR. Myocardial ischemia, reperfusion and free radical injury. Am J Cardiol 1990;65:14I23I. [PubMed: 2294677]

801. Lundberg JO, Hellstrom PM, Lundberg JM, Alving K. Greatly increased luminal nitric oxide in ulcerative colitis. Lancet 1994;344:1673-1674. [PubMed: 7996962]

802. Luoma JS, Stralin P, Marklund SL, Hiltunen TP, Sarkioja T, Yla-Herttuala S. Expression of extracellular SOD and iNOS in macrophages and smooth muscle cells in human and rabbit atherosclerotic lesions: colocalization with epitopes characteristic of oxidized LDL and peroxynitrite-modified proteins. Arterioscler Thromb Vasc Biol 1998;18:157-167. [PubMed: 9484979]

803. Lush CW, Cepinskas G, Kvietys PR. Regulation of intestinal nuclear factor-kappaB activity and Eselectin expression during sepsis: a role for peroxynitrite. Gastroenterology 2003;124:118-128. [PubMed: 12512036]

804. Luth HJ, Holzer M, Gartner U, Staufenbiel M, Arendt T. Expression of endothelial and inducible NOS-isoforms is increased in Alzheimer's disease, in APP23 transgenic mice and after experimental brain lesion in rat: evidence for an induction by amyloid pathology. Brain Res 2001;913:57-67. [PubMed: 11532247]

805. Luth HJ, Holzer M, Gertz HJ, Arendt T. Aberrant expression of nNOS in pyramidal neurons in Alzheimer's disease is highly co-localized with p21ras and p16INK4a. Brain Res 2000;852:45-55. [PubMed: 10661494]

806. Luth HJ, Munch G, Arendt T. Aberrant expression of NOS isoforms in Alzheimer's disease is structurally related to nitrotyrosine formation. Brain Res 2002;953:135-143. [PubMed: 12384247]

807. Lyall F, Gibson JL, Greer IA, Brockman DE, Eis AL, Myatt L. Increased nitrotyrosine in the diabetic placenta: evidence for oxidative stress. Diabetes Care 1998;21:1753-1758. [PubMed: 9773743]

808. Lymar S, Wishart J. Reactivity of peroxynitrite: implications for hanford waste management and remediation. Environmental Management Science Program Project 1998:59982. 
809. Lymar SV, Hurst JK. Carbon dioxide: physiological catalyst for peroxynitrite-mediated cellular damage or cellular protectant? Chem Res Toxicol 1996;9:845-850. [PubMed: 8828919]

810. Lymar SV, Hurst JK. Role of compartmentation in promoting toxicity of leukocyte-generated strong oxidants. Chem Res Toxicol 1995;8:833-840. [PubMed: 7492732]

811. Lymar SV, Jiang Q, Hurst JK. Mechanism of carbon dioxide-catalyzed oxidation of tyrosine by peroxynitrite. Biochemistry 1996;35:7855-7861. [PubMed: 8672486]

812. Lymar SV, Khairutdinov RF, Hurst JK. Hydroxyl radical formation by O-O bond homolysis in peroxynitrous acid. Inorg Chem 2003;42:5259-5266. [PubMed: 12924897]

813. Ma N, Adachi Y, Hiraku Y, Horiki N, Horiike S, Imoto I, Pinlaor S, Murata M, Semba R, Kawanishi S. Accumulation of 8-nitroguanine in human gastric epithelium induced by Helicobacter pylori infection. Biochem Biophys Res Commun 2004;319:506-510. [PubMed: 15178435]

814. Ma N, Tagawa T, Hiraku Y, Murata M, Ding X, Kawanishi S. 8-Nitroguanine formation in oral leukoplakia, a premalignant lesion. Nitric Oxide 2006;14:137-143. [PubMed: 16290060]

815. Ma XL, Gao F, Nelson AH, Lopez BL, Christopher TA, Yue TL, Barone FC. Oxidative inactivation of nitric oxide and endothelial dysfunction in stroke-prone spontaneous hypertensive rats. $\mathrm{J}$ Pharmacol Exp Ther 2001;298:879-885. [PubMed: 11504780]

816. Ma XL, Lopez BL, Liu GL, Christopher TA, Ischiropoulos H. Peroxynitrite aggravates myocardial reperfusion injury in the isolated perfused rat heart. Cardiovasc Res 1997;36:195-204. [PubMed: 9463631]

817. Maas AI, Marmarou A, Murray GD, Steyerberg EW. Clinical trials in traumatic brain injury: current problems and future solutions. Acta Neurochir Suppl 2004;89:113-118. [PubMed: 15335110]

818. Mabley JG, Jagtap P, Perretti M, Getting SJ, Salzman AL, Virag L, Szabo E, Soriano FG, Liaudet L, Abdelkarim GE, Hasko G, Marton A, Southan GJ, Szabo C. Anti-inflammatory effects of a novel, potent inhibitor of poly(ADP-ribose) polymerase. Inflamm Res 2001;50:561-569. [PubMed: 11766996]

819. Mabley JG, Liaudet L, Pacher P, Southan GJ, Groves JT, Salzman AL, Szabo C. Part II: beneficial effects of the peroxynitrite decomposition catalyst FP15 in murine models of arthritis and colitis. Mol Med 2002;8:581-590. [PubMed: 12477968]

820. Mabley JG, Pacher P, Bai P, Wallace R, Goonesekera S, Virag L, Southan GJ, Szabo C. Suppression of intestinal polyposis in Apcmin/+mice by targeting the nitric oxide or poly(ADP-ribose) pathways. Mutat Res 2004;548:107-116. [PubMed: 15063141]

821. Mabley JG, Soriano FG. Role of nitrosative stress and poly-(ADP-ribose) polymerase activation in diabetic vascular dysfunction. Curr Vasc Pharmacol 2005;3:247-252. [PubMed: 16026321]

822. Mabley JG, Southan GJ, Salzman AL, Szabo C. The combined inducible nitric oxide synthase inhibitor and free radical scavenger guanidinoethyldisulfide prevents multiple low-dose streptozotocin-induced diabetes in vivo and interleukin-1beta-induced suppression of islet insulin secretion in vitro. Pancreas 2004;28:E39-44. [PubMed: 15028959]

823. Maccaglia A, Mallozzi C, Minetti M. Differential effects of quercetin and resveratrol on Band 3 tyrosine phosphorylation signalling of red blood cells. Biochem Biophys Res Commun 2003;305:541-547. [PubMed: 12763027]

824. Macfadyen AJ, Reiter C, Zhuang Y, Beckman JS. A novel superoxide dismutase-based trap for peroxynitrite used to detect entry of peroxynitrite into erythrocyte ghosts. Chem Res Toxicol 1999;12:223-229. [PubMed: 10077484]

825. MacMicking JD, Nathan C, Hom G, Chartrain N, Fletcher DS, Trumbauer M, Stevens K, Xie QW, Sokol K, Hutchinson N, Chen H, Mudget JS. Altered responses to bacterial infection and endotoxic shock in mice lacking inducible nitric oxide synthase. Cell 1995;81:641-650. [PubMed: 7538909]

826. MacMillan-Crow LA, Crow JP, Kerby JD, Beckman JS, Thompson JA. Nitration and inactivation of manganese superoxide dismutase in chronic rejection of human renal allografts. Proc Natl Acad Sci USA 1996;93:11853-11858. [PubMed: 8876227]

827. MacMillan-Crow LA, Cruthirds DL. Invited review: manganese superoxide dismutase in disease. Free Radic Res 2001;34:325-336. [PubMed: 11328670]

828. MacMillan-Crow LA, Cruthirds DL, Ahki KM, Sanders PW, Thompson JA. Mitochondrial tyrosine nitration precedes chronic allograft nephropathy. Free Radic Biol Med 2001;31:1603-1608. [PubMed: 11744334] 
829. MacMillan-Crow LA, Greendorfer JS, Vickers SM, Thompson JA. Tyrosine nitration of c-SRC tyrosine kinase in human pancreatic ductal adenocarcinoma. Arch Biochem Biophys 2000;377:350-356. [PubMed: 10845713]

830. MacMillan-Crow LA, Thompson JA. Tyrosine modifications and inactivation of active site manganese superoxide dismutase mutant (Y34F) by peroxynitrite. Arch Biochem Biophys 1999;366:82-88. [PubMed: 10334867]

831. Madesh M, Ramachandran A, Pulimood A, Vadranam M, Balasubramanian KA. Attenuation of intestinal ischemia/reperfusion injury with sodium nitroprusside: studies on mitochondrial function and lipid changes. Biochim Biophys Acta 2000;1500:204-216. [PubMed: 10657590]

832. Mahoney LR. Evidence for the formation of hydroxyl radical in the isomerization of pernitrous acid to nitric acid in aqueous solution. J Am Chem Soc 1970;92:5262-5263.

833. Maiese K, Boccone L. Neuroprotection by peptide growth factors against anoxia and nitric oxide toxicity requires modulation of protein kinase C. J Cereb Blood Flow Metab 1995;15:440-449. [PubMed: 7714002]

834. Malinski T, Bailey F, Zhang ZG, Chopp M. Nitric oxide measured by a porphyrinic microsensor in rat brain after transient middle cerebral artery occlusion. J Cereb Blood Flow Metab 1993;13:355358. [PubMed: 8478395]

835. Malle E, Waeg G, Schreiber R, Grone EF, Sattler W, Grone HJ. Immunohistochemical evidence for the myeloperoxidase $/ \mathrm{H}_{2} \mathrm{O}_{2}$ /halide system in human atherosclerotic lesions: colocalization of myeloperoxidase and hypochlorite-modified proteins. Eur J Biochem 2000;267:4495-4503. [PubMed: 10880973]

836. Mallozzi C, De Franceschi L, Brugnara C, Di Stasi AM. Protein phosphatase 1alpha is tyrosinephosphorylated and inactivated by peroxynitrite in erythrocytes through the src family kinase fgr. Free Radic Biol Med 2005;38:1625-1636. [PubMed: 15917191]

837. Mallozzi C, Di Stasi AM, Minetti M. Activation of src tyrosine kinases by peroxynitrite. FEBS Lett 1999;456:201-206. [PubMed: 10452558]

838. Mallozzi C, Di Stasi AM, Minetti M. Nitrotyrosine mimics phosphotyrosine binding to the SH2 domain of the src family tyrosine kinase lyn. FEBS Lett 2001;503:189-195. [PubMed: 11513880]

839. Mallozzi C, Di Stasi AM, Minetti M. Peroxynitrite modulates tyrosine-dependent signal transduction pathway of human erythrocyte band 3. FASEB J 1997;11:1281-1290. [PubMed: 9409547]

840. Mallozzi C, Di Stasi MA, Minetti M. Peroxynitrite-dependent activation of src tyrosine kinases lyn and hck in erythrocytes is under mechanistically different pathways of redox control. Free Radic Biol Med 2001;30:1108-1117. [PubMed: 11369500]

841. Maneen MI, Hannah R, Vitullo L, Delance N, Cipolla MJ. Peroxynitrite diminishes myogenic activity and is associated with decreased vascular smooth muscle F-actin in rat posterior cerebral arteries. Stroke 2006;37:894-899. [PubMed: 16456123]

842. Mapp PI, Klocke R, Walsh DA, Chana JK, Stevens CR, Gallagher PJ, Blake DR. Localization of 3-nitrotyrosine to rheumatoid and normal synovium. Arthritis Rheum 2001;44:1534-1539. [PubMed: 11465704]

843. Marcillat O, Zhang Y, Davies KJ. Oxidative and non-oxidative mechanisms in the inactivation of cardiac mitochondrial electron transport chain components by doxorubicin. Biochem $\mathrm{J}$ 1989;259:181-189. [PubMed: 2719642]

844. Marcondes S, Turko IV, Murad F. Nitration of succinyl-CoA:3-oxoacid CoA-transferase in rats after endotoxin administration. Proc Natl Acad Sci USA 2001;98:7146-7151. [PubMed: 11416199]

845. Markesbery WR. Oxidative stress hypothesis in Alzheimer's disease. Free Radic Biol Med 1997;23:134-147. [PubMed: 9165306]

846. Marks DS, Vita JA, Folts JD, Keaney JF Jr, Welch GN, Loscalzo J. Inhibition of neointimal proliferation in rabbits after vascular injury by a single treatment with a protein adduct of nitric oxide. J Clin Invest 1995;96:2630-2638. [PubMed: 8675628]

847. Marshall KA, Reist M, Jenner P, Halliwell B. The neuronal toxicity of sulfite plus peroxynitrite is enhanced by glutathione depletion: implications for Parkinson's disease. Free Radic Biol Med 1999;27:515-520. [PubMed: 10490270]

848. Martindale JL, Holbrook NJ. Cellular response to oxidative stress: signaling for suicide and survival. J Cell Physiol 2002;192:1-15. [PubMed: 12115731] 
849. Massion PB, Feron O, Dessy C, Balligand JL. Nitric oxide and cardiac function: ten years after, continuing. Circ Res 2003;93:388-398. [PubMed: 12958142]

850. Matata BM, Galinanes M. Cardiopulmonary bypass exacerbates oxidative stress but does not increase proinflammatory cytokine release in patients with diabetes compared with patients without diabetes: regulatory effects of exogenous nitric oxide. J Thorac Cardiovasc Surg 2000;120:1-11. [PubMed: 10884648]

851. Matata BM, Galinanes M. Peroxynitrite is an essential component of cytokines production mechanism in human monocytes through modulation of nuclear factor-kappa B DNA binding activity. J Biol Chem 2002;277:2330-2335. [PubMed: 11706022]

852. Mathru M, Lang JD. Endothelial dysfunction in trauma patients: a preliminary communication. Shock 2005;24:210-213. [PubMed: 16135958]

853. Matsushita H, Chang E, Glassford AJ, Cooke JP, Chiu CP, Tsao PS. eNOS activity is reduced in senescent human endothelial cells: preservation by hTERT immortalization. Circ Res 2001;89:793798. [PubMed: 11679409]

854. Mayer B, Schrammel A, Klatt P, Koesling D, Schmidt K. Peroxynitrite-induced accumulation of cyclic GMP in endothelial cells and stimulation of purified soluble guanylyl cyclase. Dependence on glutathione and possible role of $S$-nitrosation. J Biol Chem 1995;270:17355-17360. [PubMed: 7615539]

855. McCall TB, Boughton-Smith NK, Palmer RMJ, Whittle BJR, Moncada S. Synthesis of nitric oxide from L-arginine by neutrophils. Biochem J 1989;261:293-296. [PubMed: 2549965]

856. McCartney-Francis N, Allen JB, Mizel DE, Albina JE, Xie QW, Nathan CF, Wahl SM. Suppression of arthritis by an inhibitor of nitric oxide synthase. J Exp Med 1993;178:749-754. [PubMed: 7688035]

857. McCartney-Francis NL, Song XY, Mizel DE, Wahl CL, Wahl SM. Hemoglobin protects from streptococcal cell wall-induced arthritis. Arthritis Rheum 1999;42:1119-1127. [PubMed: 10366104]

858. McCord JM. Oxygen-derived free radicals in postischemic tissue injury. N Engl J Med 1985;312:159-163. [PubMed: 2981404]

859. McCord JM, Fridovich I. Superoxide dismutase: an enzymic function for erythrocuprein (hemocuprein). J Biol Chem 1969;244:6049-6055. [PubMed: 5389100]

860. McInnes IB, Leung BP, Field M, Wei XQ, Huang FP, Sturrock RD, Kinninmonth A, Weidner J, Mumford R, Liew FY. Production of nitric oxide in the synovial membrane of rheumatoid and osteoarthritis patients. J Exp Med 1996;184:1519-1524. [PubMed: 8879223]

861. McLean GW, Carragher NO, Avizienyte E, Evans J, Brunton VG, Frame MC. The role of focaladhesion kinase in cancer: a new therapeutic opportunity. Nat Rev Cancer 2005;5:505-515. [PubMed: 16069815]

862. Meade MO, Granton JT, Matte-Martyn A, McRae K, Weaver B, Cripps P, Keshavjee SH. A randomized trial of inhaled nitric oxide to prevent ischemia-reperfusion injury after lung transplantation. Am J Respir Crit Care Med 2003;167:1483-1489. [PubMed: 12770854]

863. Meguro M, Katsuramaki T, Kimura H, Isobe M, Nagayama M, Kukita K, Nui A, Hirata K. Apoptosis and necrosis after warm ischemia-reperfusion injury of the pig liver and their inhibition by ONO-1714. Transplantation 2003;75:703-710. [PubMed: 12640313]

864. Mehlhorn U, Krahwinkel A, Geissler HJ, LaRosee K, Fischer UM, Klass O, Suedkamp M, Hekmat $\mathrm{K}$, Tossios P, Bloch W. Nitrotyrosine and 8-isoprostane formation indicate free radical-mediated injury in hearts of patients subjected to cardioplegia. J Thorac Cardiovasc Surg 2003;125:178-183. [PubMed: 12539002]

865. Melichar VO, Behr-Roussel D, Zabel U, Uttenthal LO, Rodrigo J, Rupin A, Verbeuren TJ, Kumar HSA, Schmidt HH. Reduced cGMP signaling associated with neointimal proliferation and vascular dysfunction in late-stage atherosclerosis. Proc Natl Acad Sci USA 2004;101:16671-16676. [PubMed: 15546990]

866. Melone MA, Jori FP, Peluso G. Huntington's disease: new frontiers for molecular and cell therapy. Curr Drug Targets 2005;6:43-56. [PubMed: 15720212] 
867. Menconi MJ, Unno N, Smith M, Aguirre DE, Fink MP. Nitric oxide donor-induced hyperpermeability of cultured intestinal epithelial monolayers: role of superoxide radical, hydroxyl radical, peroxynitrite. Biochim Biophys Acta 1998;1425:189-203. [PubMed: 9813320]

868. Merényi G, Lind J, Goldstein S, Czapski G. Mechanism and thermochemistry of peroxynitrite decomposition in water. J Phys Chem 1999;103:3085-3091.

869. Merrill G, McConnell P, Vandyke K, Powell S. Coronary and myocardial effects of acetaminophen: protection during ischemia-reperfusion. Am J Physiol Heart Circ Physiol 2001;280:H2631-H2638. [PubMed: 11356619]

870. Merrill GF. Acetaminophen and low-flow myocardial ischemia: efficacy and antioxidant mechanisms. Am J Physiol Heart Circ Physiol 2002;282:H1341-H1349. [PubMed: 11893570]

871. Merrill GF, Goldberg E. Antioxidant properties of acetaminophen and cardioprotection. Basic Res Cardiol 2001;96:423-430. [PubMed: 11605988]

872. Mhatre M, Floyd RA, Hensley K. Oxidative stress and neuroinflammation in Alzheimer's disease and amyotrophic lateral sclerosis: common links and potential therapeutic targets. J Alzheimers Dis 2004;6:147-157. [PubMed: 15096698]

873. Michelson AM, Maral J. Carbonate anions: effects on the oxidation of luminol, oxidative hemolysis, $\gamma$-irradiation and the reaction of activated oxygen species with enzymes containing various active centres. Biochomie 1983;65:95-104.

874. Middleton SJ, Shorthouse M, Hunter JO. Increased nitric oxide synthesis in ulcerative colitis. Lancet 1993;341:465-466. [PubMed: 8094492]

875. Miesel R, Kurpisz M, Kroger H. Modulation of inflammatory arthritis by inhibition of poly(ADP ribose) polymerase. Inflammation 1995;19:379-387. [PubMed: 7628865]

876. Migita K. Nitric oxide in rheumatoid arthritis. Nippon Rinsho 2005;63(Suppl 1):106-109. [PubMed: 15799327]

877. Mihm MJ, Bauer JA. Peroxynitrite-induced inhibition and nitration of cardiac myofibrillar creatine kinase. Biochimie 2002;84:1013-1019. [PubMed: 12504281]

878. Mihm MJ, Coyle CM, Schanbacher BL, Weinstein DM, Bauer JA. Peroxynitrite induced nitration and inactivation of myofibrillar creatine kinase in experimental heart failure. Cardiovasc Res 2001;49:798-807. [PubMed: 11230979]

879. Mihm MJ, Jing L, Bauer JA. Nitrotyrosine causes selective vascular endothelial dysfunction and DNA damage. J Cardiovasc Pharmacol 2000;36:182-187. [PubMed: 10942159]

880. Mihm MJ, Wattanapitayakul SK, Piao SF, Hoyt DG, Bauer JA. Effects of angiotensin II on vascular endothelial cells: formation of receptor-mediated reactive nitrogen species. Biochem Pharmacol 2003;65:1189-1197. [PubMed: 12663054]

881. Mihm MJ, Yu F, Carnes CA, Reiser PJ, McCarthy PM, Van Wagoner DR, Bauer JA. Impaired myofibrillar energetics and oxidative injury during human atrial fibrillation. Circulation 2001;104:174-180. [PubMed: 11447082]

882. Mihm MJ, Yu F, Reiser PJ, Bauer JA. Effects of peroxynitrite on isolated cardiac trabeculae: selective impact on myofibrillar energetic controllers. Biochimie 2003;85:587-596. [PubMed: 12829376]

883. Mihm MJ, Yu F, Weinstein DM, Reiser PJ, Bauer JA. Intracellular distribution of peroxynitrite during doxorubicin cardiomyopathy: evidence for selective impairment of myofibrillar creatine kinase. Br J Pharmacol 2002;135:581-588. [PubMed: 11834605]

884. Mikami S, Kawashima S, Kanazawa K, Hirata K, Katayama Y, Hotta H, Hayashi Y, Ito H, Yokoyama M. Expression of nitric oxide synthase in a murine model of viral myocarditis induced by coxsackievirus B3. Biochem Biophys Res Commun 1996;220:983-989. [PubMed: 8607880]

885. Miller MJ, Thompson JH, Zhang XJ, Sadowska-Krowicka H, Kakkis JL, Munshi UK, Sandoval M, Rossi JL, Eloby-Childress S, Beckman JS, Ye YZ, Rodi CP, Manning PT, Currie MG, Clark DA. Role of inducible nitric oxide synthase expression and peroxynitrite formation in guinea pig ileitis. Gastroenterology 1995;109:1475-1483. [PubMed: 7557128]

886. Minchenko AG, Stevens MJ, White L, Abatan OI, Komjati K, Pacher P, Szabo C, Obrosova IG. Diabetes-induced overexpression of endothelin-1 and endothelin receptors in the rat renal cortex is mediated via poly(ADP-ribose) polymerase activation. FASEB J 2003;17:1514-1516. [PubMed: 12824290] 
887. Minetti M, Mallozzi C, Di Stasi AM. Peroxynitrite activates kinases of the src family and upregulates tyrosine phosphorylation signaling. Free Radic Biol Med 2002;33:744-754. [PubMed: 12208363]

888. Mohr S, Stamler JS, Brune B. Mechanism of covalent modification of glyceraldehyde-3-phosphate dehydrogenase at its active site thiol by nitric oxide, peroxynitrite and related nitrosating agents. FEBS Lett 1994;348:223-227. [PubMed: 8034046]

889. Mollnau H, Wendt M, Szocs K, Lassegue B, Schulz E, Oelze M, Li H, Bodenschatz M, August M, Kleschyov AL, Tsilimingas N, Walter U, Forstermann U, Meinertz T, Griendling K, Munzel T. Effects of angiotensin II infusion on the expression and function of NAD(P)H oxidase and components of nitric oxide/cGMP signaling. Circ Res 2002;90:E58-65. [PubMed: 11884382]

890. Moncada S, Bolanos JP. Nitric oxide, cell bioenergetics and neurodegeneration. J Neurochem 2006;97:1676-1689. [PubMed: 16805776]

891. Moncada S, Erusalimsky JD. Does nitric oxide modulate mitochondrial energy generation and apoptosis? Nat Rev Mol Cell Biol 2002;3:214-220. [PubMed: 11994742]

892. Moncada S, Herman AG, Vanhoutte PM. Endothelium-derived relaxing factor is identified as nitric oxide. Trends Pharmacol Sci 1987;8:365-368.

893. Moncada S, Higgs EA. The discovery of nitric oxide and its role in vascular biology. Br J Pharmacol 2006;147(Suppl 1):S193-S201. [PubMed: 16402104]

894. Moncada S, Palmer RM, Gryglewski RJ. Mechanism of action of some inhibitors of endotheliumderived relaxing factor. Proc Natl Acad Sci USA 1986;83:9164-9168. [PubMed: 3024168]

895. Moncada S, Palmer RMJ, Higgs EA. Nitric oxide: physiology, pathophysiology, pharmacology. Pharmacol Rev 1991;43:109-142. [PubMed: 1852778]

896. Mondoro TH, Shafer BC, Vostal JG. Peroxynitrite-induced tyrosine nitration and phosphorylation in human platelets. Free Radic Biol Med 1997;22:1055-1063. [PubMed: 9034245]

897. Montague PR, Gally JA, Edelman GM. Spatial signaling in the development and function of neural connections. Cereb Cortex 1991;1:199-220. [PubMed: 1822733]

898. Moochhala SM, Lu J, Xing MC, Anuar F, Ng KC, Yang KL, Whiteman M, Atan S. Mercaptoethylguanidine inhibition of inducible nitric oxide synthase and cyclooxygenase-2 expressions induced in rats after fluid-percussion brain injury. J Trauma 2005;59:450-457. [PubMed: 16294091]

899. Moore KP, Darley-Usmar V, Morrow J, Roberts LJ 2nd. Formation of F2-isoprostanes during oxidation of human low-density lipoprotein and plasma by peroxynitrite. Circ Res 1995;77:335341. [PubMed: 7614720]

900. Moreira PI, Siedlak SL, Aliev G, Zhu X, Cash AD, Smith MA, Perry G. Oxidative stress mechanisms and potential therapeutics in Alzheimer disease. J Neural Transm 2005;112:921-932. [PubMed: 15583960]

901. Moreira PI, Smith MA, Zhu X, Nunomura A, Castellani RJ, Perry G. Oxidative stress and neurodegeneration. Ann NY Acad Sci 2005;1043:545-552. [PubMed: 16037277]

902. Mori E, Haramaki N, Ikeda H, Imaizumi T. Intra-coronary administration of L-arginine aggravates myocardial stunning through production of peroxynitrite in dogs. Cardiovasc Res 1998;40:113123. [PubMed: 9876323]

903. Moriel P, Abdalla DS. Nitrotyrosine bound to beta-VLDL-apoproteins: a biomarker of peroxynitrite formation in experimental atherosclerosis. Biochem Biophys Res Commun 1997;232:332-335. [PubMed: 9125175]

904. Morisue A, Wakabayashi G, Shimazu M, Tanabe M, Mukai M, Matsumoto K, Kawachi S, Yoshida M, Yamamoto S, Kitajima M. The role of nitric oxide after a short period of liver ischemiareperfusion. J Surg Res 2003;109:101-109. [PubMed: 12643850]

905. Morita H, Seidman J, Seidman CE. Genetic causes of human heart failure. J Clin Invest 2005;115:518-526. [PubMed: 15765133]

906. Moro MA, Almeida A, Bolanos JP, Lizasoain I. Mitochondrial respiratory chain and free radical generation in stroke. Free Radic Biol Med 2005;39:1291-1304. [PubMed: 16257638]

907. Moro MA, Cardenas A, Hurtado O, Leza JC, Lizasoain I. Role of nitric oxide after brain ischaemia. Cell Calcium 2004;36:265-275. [PubMed: 15261482] 
908. Moro MA, Darley-Usmar VM, Goodwin DA, Read NG, Zamora-Pino R, Feelisch M, Radomski MW, Moncada S. Paradoxical fate and biological action of peroxynitrite on human platelets. Proc Natl Acad Sci USA 1994;91:6702-6706. [PubMed: 7517561]

909. Moro MA, Darley-Usmar VM, Lizasoain I, Su Y, Knowles RG, Radomski MW, Moncada S. The formation of nitric oxide donors from peroxynitrite. Br J Pharmacol 1995;116:1999-2004. [PubMed: 8640338]

910. Morten KJ, Ackrell BA, Melov S. Mitochondrial reactive oxygen species in mice lacking superoxide dismutase 2: attenuation via antioxidant treatment. J Biol Chem 2006;281:3354-3359. [PubMed: 16326710]

911. Mota-Filipe H, McDonald MC, Cuzzocrea S, Thiemermann C. A membrane-permeable radical scavenger reduces the organ injury in hemorrhagic shock. Shock 1999;12:255-261. [PubMed: 10509626]

912. Mujumdar VS, Aru GM, Tyagi SC. Induction of oxidative stress by homocyst(e)ine impairs endothelial function. J Cell Biochem 2001;82:491-500. [PubMed: 11500925]

913. Mungrue IN, Gros R, You X, Pirani A, Azad A, Csont T, Schulz R, Butany J, Stewart DJ, Husain M. Cardiomyocyte overexpression of iNOS in mice results in peroxynitrite generation, heart block, sudden death. J Clin Invest 2002;109:735-743. [PubMed: 11901182]

914. Mungrue IN, Stewart DJ, Husain M. The Janus faces of iNOS. Circ Res 2003;93:e74. [PubMed: 14525922]

915. Murakami K, Kondo T, Kawase M, Li Y, Sato S, Chen SF, Chan PH. Mitochondrial susceptibility to oxidative stress exacerbates cerebral infarction that follows permanent focal cerebral ischemia in mutant mice with manganese superoxide dismutase deficiency. J Neurosci 1998;18:205-213. [PubMed: 9412501]

916. Muriel P, Castaneda G, Ortega M, Noel F. Insights into the mechanism of erythrocyte $\mathrm{Na}^{+} / \mathrm{K}^{+}$ATPase inhibition by nitric oxide and peroxynitrite anion. J Appl Toxicol 2003;23:275-278. [PubMed: 12884412]

917. Muriel P, Sandoval G. Hepatic basolateral plasma high-affinity $\mathrm{Ca}^{2+}$-ATPase is inhibited by nitric oxide and peroxynitrite anion. J Appl Toxicol 2000;20:435-439. [PubMed: 11180264]

918. Muriel P, Sandoval G. Nitric oxide and peroxynitrite anion modulate liver plasma membrane fluidity and $\mathrm{Na}(+) / \mathrm{K}(+)-A T P a s e$ activity. Nitric Oxide 2000;4:333-342. [PubMed: 10944417]

919. Murray J, Taylor SW, Zhang B, Ghosh SS, Capaldi RA. Oxidative damage to mitochondrial complex I due to peroxynitrite: identification of reactive tyrosines by mass spectrometry. J Biol Chem 2003;278:37223-37230. [PubMed: 12857734]

920. Murry CE, Jennings RB, Reimer KA. Preconditioning with ischemia: a delay of lethal cell injury in ischemic myocardium. Circulation 1986;74:1124-1136. [PubMed: 3769170]

921. Muscoli C, Sacco I, Alecce W, Palma E, Nistico R, Costa N, Clementi F, Rotiroti D, Romeo F, Salvemini D, Mehta JL, Mollace V. The protective effect of superoxide dismutase mimetic M40401 on balloon injury-related neointima formation: role of the lectin-like oxidized low-density lipoprotein receptor-1. J Pharmacol Exp Ther 2004;311:44-50. [PubMed: 15220383]

922. Nabeyrat E, Jones GE, Fenwick PS, Barnes PJ, Donnelly LE. Mitogen-activated protein kinases mediate peroxynitrite-induced cell death in human bronchial epithelial cells. Am J Physiol Lung Cell Mol Physiol 2003;284:L1112-L1120. [PubMed: 12598225]

923. Nagayama T, Simon RP, Chen D, Henshall DC, Pei W, Stetler RA, Chen J. Activation of poly(ADPribose) polymerase in the rat hippocampus may contribute to cellular recovery following sub-lethal transient global ischemia. J Neurochem 2000;74:1636-1645. [PubMed: 10737622]

924. Naidu BV, Farivar AS, Woolley SM, Fraga C, Salzman AL, Szabo C, Groves JT, Mulligan MS. Enhanced peroxynitrite decomposition protects against experimental obliterative bronchiolitis. Exp Mol Pathol 2003;75:12-17. [PubMed: 12834621]

925. Naidu BV, Fraga C, Salzman AL, Szabo C, Verrier ED, Mulligan MS. Critical role of reactive nitrogen species in lung ischemia-reperfusion injury. J Heart Lung Transplant 2003;22:784-793. [PubMed: 12873547]

926. Naito Y, Takagi T, Ichikawa H, Tomatsuri N, Kuroda M, Isozaki Y, Katada K, Uchiyama K, Kokura S, Yoshida N, Okanoue T, Yoshikawa T. A novel potent inhibitor of inducible nitric oxide inhibitor, 
ONO-1714, reduces intestinal ischemia-reperfusion injury in rats. Nitric Oxide 2004;10:170-177. [PubMed: 15158697]

927. Najjar SS, Scuteri A, Lakatta EG. Arterial aging: is it an immutable cardiovascular risk factor? Hypertension 2005;46:454-462. [PubMed: 16103272]

928. Nakajima A, Ueda K, Takaoka M, Yoshimi Y, Matsumura Y. Opposite effects of pre- and postischemic treatments with nitric oxide donor on ischemia/reperfusion-induced renal injury. $\mathrm{J}$ Pharmacol Exp Ther 2006;316:1038-1046. [PubMed: 16306274]

929. Nakamura H, Tsukada H, Oya M, Onomura M, Saito T, Fukuda K, Kodama M, Taniguchi T, Tominaga M, Hosokawa M, Seino Y. Aminoguanidine has both an anti-inflammatory effect on experimental colitis and a proliferative effect on colonic mucosal cells. Scand J Gastroenterol 1999;34:1117-1122. [PubMed: 10582763]

930. Naoi M, Maruyama W. Future of neuroprotection in Parkinson's disease. Parkinsonism Relat Disord 2001;8:139-145. [PubMed: 11489679]

931. Napoli C, de Nigris F, Williams-Ignarro S, Pignalosa O, Sica V, Ignarro LJ. Nitric oxide and atherosclerosis: an update. Nitric Oxide. In press

932. Naruse K, Shimizu K, Muramatsu M, Toki Y, Miyazaki Y, Okumura K, Hashimoto H, Ito T. Longterm inhibition of NO synthesis promotes atherosclerosis in the hypercholesterolemic rabbit thoracic aorta. $\mathrm{PGH}_{2}$ does not contribute to impaired endothelium-dependent relaxation. Arterioscler Thromb 1994;14:746-752. [PubMed: 8172852]

933. Nathan C. Specificity of a third kind: reactive oxygen and nitrogen intermediates in cell signaling. J Clin Invest 2003;111:769-778. [PubMed: 12639979]

934. Nathan C, Xie QW. Nitric oxide synthases: roles, tolls, controls. Cell 1994;78:915-918. [PubMed: 7522969]

935. Nathan C, Xie QW. Regulation of biosynthesis of nitric oxide. J Biol Chem 1994;269:13725-13728. [PubMed: 7514592]

936. Nathan CF, Hibbs JBJ. Role of nitric oxide synthesis in macrophage antimicrobial activity. Curr Opin Immunol 1991;3:65-70. [PubMed: 1711326]

937. Nauser T, Koppenol WH. The rate constant of the reaction of superoxide with nitrogen monoxide: approaching the diffusion limit. J Phys Chem A 2002;106:4084-4086.

938. Navarro A, Gomez C, Lopez-Cepero JM, Boveris A. Beneficial effects of moderate exercise on mice aging: survival, behavior, oxidative stress, mitochondrial electron transfer. Am J Physiol Regul Integr Comp Physiol 2004;286:R505-R511. [PubMed: 14615275]

939. Neeley WL, Delaney JC, Henderson PT, Essigmann JM. In vivo bypass efficiencies and mutational signatures of the guanine oxidation products 2-aminoimidazolone and 5-guanidino-4-nitroimidazole. J Biol Chem 2004;279:43568-43573. [PubMed: 15299010]

940. Neumann P, Gertzberg N, Vaughan E, Weisbrot J, Woodburn R, Lambert W, Johnson A. Peroxynitrite mediates TNF- $\alpha$-induced endothelial barrier dysfunction and nitration of actin. Am J Physiol Lung Cell Mol Physiol 2006;290:L674-L684. [PubMed: 16284212]

941. Newman DK, Hoffman S, Kotamraju S, Zhao T, Wakim B, Kalyanaraman B, Newman PJ. Nitration of PECAM-1 ITIM tyrosines abrogates phosphorylation and SHP-2 binding. Biochem Biophys Res Commun 2002;296:1171-1179. [PubMed: 12207897]

942. Newmeyer DD, Ferguson-Miller S. Mitochondria: releasing power for life and unleashing the machineries of death. Cell 2003;112:481-490. [PubMed: 12600312]

943. Nielsen VG, Crow JP, Mogal A, Zhou F, Parks DA. Peroxynitrite decreases hemostasis in human plasma in vitro. Anesth Analg 2004;99:21-26. [PubMed: 15281495]

944. Nieuwenhuijzen GA, Goris RJ. The gut: the "motor" of multiple organ dysfunction syndrome? Curr Opin Clin Nutr Metab Care 1999;2:399-404. [PubMed: 10589382]

945. Niles JC, Wishnok JS, Tannenbaum SR. Peroxynitrite-induced oxidation and nitration products of guanine and 8-oxoguanine: structures and mechanisms of product formation. Nitric Oxide 2006;14:109-121. [PubMed: 16352449]

946. Nishikawa T, Edelstein D, Brownlee M. The missing link: a single unifying mechanism for diabetic complications. Kidney Int Suppl 2000;77:S26-S30. [PubMed: 10997687]

947. Nohl H. Generation of superoxide radicals as byproduct of cellular respiration. Ann Biol Clin 1994;52:199-204. 
948. Noiri E, Peresleni T, Miller F, Goligorsky MS. In vivo targeting of inducible NO synthase with oligodeoxynucleotides protects rat kidney against ischemia. J Clin Invest 1996;97:2377-2383. [PubMed: 8636419]

949. Nomiyama T, Igarashi Y, Taka H, Mineki R, Uchida T, Ogihara T, Choi JB, Uchino H, Tanaka Y, Maegawa H, Kashiwagi A, Murayama K, Kawamori R, Watada H. Reduction of insulin-stimulated glucose uptake by peroxynitrite is concurrent with tyrosine nitration of insulin receptor substrate-1. Biochem Biophys Res Commun 2004;320:639-647. [PubMed: 15240096]

950. Nossuli TO, Hayward R, Jensen D, Scalia R, Lefer AM. Mechanisms of cardioprotection by peroxynitrite in myocardial ischemia and reperfusion injury. Am J Physiol Heart Circ Physiol 1998;275:H509-H519.

951. Nossuli TO, Hayward R, Scalia R, Lefer AM. Peroxynitrite reduces myocardial infarct size and preserves coronary endothelium after ischemia and reperfusion in cats. Circulation 1997;96:23172324. [PubMed: 9337206]

952. Nowak P, Kolodziejczyk J, Wachowicz B. Peroxynitrite and fibrinolytic system: the effect of peroxynitrite on plasmin activity. Mol Cell Biochem 2004;267:141-146. [PubMed: 15663195]

953. Nowak P, Wachowicz B. Peroxynitrite-mediated modification of fibrinogen affects platelet aggregation and adhesion. Platelets 2002;13:293-299. [PubMed: 12189015]

955. Oates JC, Gilkeson GS. The biology of nitric oxide and other reactive intermediates in systemic lupus erythematosus. Clin Immunol. In press

956. Obermeier F, Kojouharoff G, Hans W, Scholmerich J, Gross V, Falk W. Interferon-gamma (IFNgamma)- and tumour necrosis factor (TNF)-induced nitric oxide as toxic effector molecule in chronic dextran sulphate sodium (DSS)-induced colitis in mice. Clin Exp Immunol 1999;116:238245. [PubMed: 10337013]

957. Obrosova IG. Increased sorbitol pathway activity generates oxidative stress in tissue sites for diabetic complications. Antioxid Redox Signal 2005;7:1543-1552. [PubMed: 16356118]

958. Obrosova IG, Drel VR, Pacher P, Ilnytska O, Wang ZQ, Stevens MJ, Yorek MA. Oxidativenitrosative stress and poly-(ADP-ribose) polymerase (PARP) activation in experimental diabetic neuropathy: the relation is revisited. Diabetes 2005;54:3435-3441. [PubMed: 16306359]

959. Obrosova IG, Julius UA. Role for poly(ADP-ribose) polymerase activation in diabetic nephropathy, neuropathy and retinopathy. Curr Vasc Pharmacol 2005;3:267-283. [PubMed: 16026323]

960. Obrosova IG, Li F, Abatan OI, Forsell MA, Komjati K, Pacher P, Szabo C, Stevens MJ. Role of poly(ADP-ribose) polymerase activation in diabetic neuropathy. Diabetes 2004;53:711-720. [PubMed: 14988256]

961. Obrosova IG, Mabley JG, Zsengeller Z, Charniauskaya T, Abatan OI, Groves JT, Szabo C. Role for nitrosative stress in diabetic neuropathy: evidence from studies with a peroxynitrite decomposition catalyst. FASEB J 2005;19:401-403. [PubMed: 15611153]

962. Obrosova IG, Minchenko AG, Frank RN, Seigel GM, Zsengeller Z, Pacher P, Stevens MJ, Szabo C. Poly(ADP-ribose) polymerase inhibitors counteract diabetes- and hypoxia-induced retinal vascular endothelial growth factor overexpression. Int J Mol Med 2004;14:55-64. [PubMed: 15202016]

963. Obrosova IG, Pacher P, Szabo C, Zsengeller Z, Hirooka H, Stevens MJ, Yorek MA. Aldose reductase inhibition counteracts oxidative-nitrosative stress and poly(ADP-ribose) polymerase activation in tissue sites for diabetes complications. Diabetes 2005;54:234-242. [PubMed: 15616034]

963a. O’Donnell VB, Eiserich JP, Chumley PH, Jablonsky MJ, Krishna NR, Kirk M, Barnes S, DarleyUsmar VM, Freeman BA. Nitration of unsaturated fatty acids by nitric oxide-derived reactive nitrogen species peroxynitrite, nitrous acid, nitrogen dioxide, nitronium ion. Chem Res Toxicol 1999;12:83-92. [PubMed: 9894022]

964. Oeckler RA, Wolin MS. New concepts in vascular nitric oxide signaling. Curr Atheroscler Rep 2000;2:437-444. [PubMed: 11122776]

965. Oh-Hashi K, Maruyama W, Isobe K. Peroxynitrite induces GADD34, 45, 153 VIA p38 MAPK in human neuroblastoma SH-SY5Y cells. Free Radic Biol Med 2001;30:213-221. [PubMed: 11163539]

966. Ohmori H, Kanayama N. Immunogenicity of an inflammation-associated product, tyrosine nitrated self-proteins. Autoimmun Rev 2005;4:224-229. [PubMed: 15893716] 
967. Ohshima H, Friesen M, Brouet I, Bartsch H. Nitrotyrosine as a new marker for endogenous nitrosation and nitration of proteins. Food Chem Toxicol 1990;28:647-652. [PubMed: 2272563]

968. Ohtaki H, Funahashi H, Dohi K, Oguro T, Horai R, Asano M, Iwakura Y, Yin L, Matsunaga M, Goto N, Shioda S. Suppression of oxidative neuronal damage after transient middle cerebral artery occlusion in mice lacking interleukin-1. Neurosci Res 2003;45:313-324. [PubMed: 12631467]

969. Ohtsu H, Frank GD, Utsunomiya H, Eguchi S. Redox-dependent protein kinase regulation by angiotensin II: mechanistic insights and its pathophysiology. Antioxid Redox Signal 2005;7:13151326. [PubMed: 16115037]

970. Ohtsuka M, Konno F, Honda H, Oikawa T, Ishikawa M, Iwase N, Isomae K, Ishii F, Hemmi H, Sato S. PPA250 [3-(2,4-diflu-orophenyl)-6-[2-[4-(1H-imidazol-1-ylmethyl) phenoxy] ethoxy]-2phenylpyridine], a novel orally effective inhibitor of the dimerization of inducible nitric-oxide synthase, exhibits an anti-inflammatory effect in animal models of chronic arthritis. J Pharmacol Exp Ther 2002;303:52-57. [PubMed: 12235232]

971. Okamoto T, Akaike T, Sawa T, Miyamoto Y, van der Vliet A, Maeda H. Activation of matrix metalloproteinases by peroxynitrite-induced protein $S$-glutathiolation via disulfide $S$-oxide formation. J Biol Chem 2001;276:29596-29602. [PubMed: 11395496]

972. Oleszak EL, Zaczynska E, Bhattacharjee M, Butunoi C, Legido A, Katsetos CD. Inducible nitric oxide synthase and nitrotyrosine are found in monocytes/macrophages and/or astrocytes in acute, but not in chronic, multiple sclerosis. Clin Diagn Lab Immunol 1998;5:438-445. [PubMed: 9665945]

973. Oliver FJ, Menissier-de Murcia J, Nacci C, Decker P, Andriantsitohaina R, Muller S, de la Rubia G, Stoclet JC, de Murcia G. Resistance to endotoxic shock as a consequence of defective NF-kappaB activation in poly (ADP-ribose) polymerase-1 deficient mice. EMBO J 1999;18:4446-4454. [PubMed: 10449410]

974. Onozato ML, Tojo A, Goto A, Fujita T, Wilcox CS. Oxidative stress and nitric oxide synthase in rat diabetic nephropathy: effects of ACEI and ARB. Kidney Int 2002;61:186-194. [PubMed: 11786100]

975. Orucevic A, Bechberger J, Green AM, Shapiro RA, Billiar TR, Lala PK. Nitric-oxide production by murine mammary adenocarcinoma cells promotes tumor-cell invasiveness. Int J Cancer 1999;81:889-896. [PubMed: 10362135]

976. Osuka K, Feustel PJ, Mongin AA, Tranmer BI, Kimelberg HK. Tamoxifen inhibits nitrotyrosine formation after reversible middle cerebral artery occlusion in the rat. J Neurochem 2001;76:18421850. [PubMed: 11259502]

977. Ovechkin AV, Lominadze D, Sedoris KC, Gozal E, Robinson TW, Roberts AM. Inhibition of inducible nitric oxide synthase attenuates platelet adhesion in subpleural arterioles caused by lung ischemia-reperfusion in rabbits. J Appl Physiol 2005;99:2423-2432. [PubMed: 16037396]

978. Oyama J, Shimokawa H, Momii H, Cheng X, Fukuyama N, Arai Y, Egashira K, Nakazawa H, Takeshita A. Role of nitric oxide and peroxynitrite in the cytokine-induced sustained myocardial dysfunction in dogs in vivo. J Clin Invest 1998;101:2207-2214. [PubMed: 9593776]

979. Oyanagui Y. Nitric oxide and superoxide radical are involved in both initiation and development of adjuvant arthritis in rats. Life Sci 1994;54:PL285-289. [PubMed: 8164499]

980. Ozdulger A, Cinel I, Unlu A, Cinel L, Mavioglu I, Tamer L, Atik U, Oral U. Poly(ADP-ribose) synthetase inhibition prevents lipopolysaccharide-induced peroxynitrite mediated damage in diaphragm. Pharmacol Res 2002;46:67-73. [PubMed: 12208123]

981. Ozturk OH, Cetin A, Ozdem SS, Uysal N, Kayisli UA, Senturk UK, Yesilkaya A. Plasma levels of nitrites, PGF1alpha, nitrotyrosine in LPS-treated rats: functional and histochemical implications in aorta. J Physiol Biochem 2006;62:27-34. [PubMed: 16909929]

982. Pacher P, Csordas G, Hajnoczky G. Mitochondrial Ca(2+) signaling and cardiac apoptosis. Biol Signals Recept 2001;10:200-223. [PubMed: 11351129]

983. Pacher P, Cziraki A, Mabley JG, Liaudet L, Papp L, Szabo C. Role of poly(ADP-ribose) polymerase activation in endotoxin-induced cardiac collapse in rodents. Biochem Pharmacol 2002;64:17851791. [PubMed: 12445868]

984. Pacher P, Hajnoczky G. Propagation of the apoptotic signal by mitochondrial waves. EMBO J 2001;20:4107-4121. [PubMed: 11483514] 
985. Pacher P, Liaudet L, Bai P, Mabley JG, Kaminski PM, Virag L, Deb A, Szabo E, Ungvari Z, Wolin MS, Groves JT, Szabo C. Potent metalloporphyrin peroxynitrite decomposition catalyst protects against the development of doxorubicin-induced cardiac dysfunction. Circulation 2003;107:896904. [PubMed: 12591762]

986. Pacher P, Liaudet L, Bai P, Virag L, Mabley JG, Hasko G, Szabo C. Activation of poly(ADP-ribose) polymerase contributes to development of doxorubicin-induced heart failure. J Pharmacol Exp Ther 2002;300:862-867. [PubMed: 11861791]

987. Pacher P, Liaudet L, Mabley J, Komjati K, Szabo C. Pharmacologic inhibition of poly(adenosine diphosphate-ribose) polymerase may represent a novel therapeutic approach in chronic heart failure. J Am Coll Cardiol 2002;40:1006-1016. [PubMed: 12225730]

988. Pacher P, Liaudet L, Mabley JG, Cziraki A, Hasko G, Szabo C. Beneficial effects of a novel ultrapotent poly(ADP-ribose) polymerase inhibitor in murine models of heart failure. Int J Mol Med 2006;17:369-375. [PubMed: 16391839]

989. Pacher P, Liaudet L, Soriano FG, Mabley JG, Szabo E, Szabo C. The role of poly(ADP-ribose) polymerase activation in the development of myocardial and endothelial dysfunction in diabetes. Diabetes 2002;51:514-521. [PubMed: 11812763]

990. Pacher P, Mabley JG, Liaudet L, Evgenov OV, Marton A, Hasko G, Kollai M, Szabo C. Left ventricular pressure-volume relationship in a rat model of advanced aging-associated heart failure. Am J Physiol Heart Circ Physiol 2004;287:H2132-H2137. [PubMed: 15231502]

991. Pacher P, Mabley JG, Soriano FG, Liaudet L, Komjati K, Szabo C. Endothelial dysfunction in aging animals: the role of poly(ADP-ribose) polymerase activation. Br J Pharmacol 2002;135:1347-1350. [PubMed: 11906946]

992. Pacher P, Mabley JG, Soriano FG, Liaudet L, Szabo C. Activation of poly(ADP-ribose) polymerase contributes to the endothelial dysfunction associated with hypertension and aging. Int J Mol Med 2002;9:659-664. [PubMed: 12011985]

993. Pacher P, Nivorozhkin A, Szabo C. Therapeutic effects of xanthine oxidase inhibitors: renaissance half a century after the discovery of allopurinol. Pharmacol Rev 2006;58:87-114. [PubMed: 16507884]

994. Pacher P, Obrosova IG, Mabley JG, Szabo C. Role of nitrosative stress and peroxynitrite in the pathogenesis of diabetic complications. Emerging new therapeutical strategies. Curr Med Chem 2005;12:267-275. [PubMed: 15723618]

995. Pacher P, Schulz R, Liaudet L, Szabo C. Nitrosative stress and pharmacological modulation of heart failure. Trends Pharmacol Sci 2005;26:302-310. [PubMed: 15925705]

996. Pacher P, Szabo C. Role of peroxynitrite in the pathogenesis of cardiovascular complications of diabetes. Curr Opin Pharmacol 2006;6:136-141. [PubMed: 16483848]

997. Pacher P, Szabo C. Role of poly(ADP-ribose) polymerase-1 activation in the pathogenesis of diabetic complications: endothelial dysfunction, as a common underlying theme. Antioxid Redox Signal 2005;7:1568-1580. [PubMed: 16356120]

998. Pacher P, Vaslin A, Benko R, Mabley JG, Liaudet L, Hasko G, Marton A, Batkai S, Kollai M, Szabo C. A new, potent poly-(ADP-ribose) polymerase inhibitor improves cardiac and vascular dysfunction associated with advanced aging. J Pharmacol Exp Ther 2004;311:485-491. [PubMed: 15213249]

999. Packer MA, Murphy MP. Peroxynitrite causes calcium efflux from mitochondria which is prevented by cyclosporin A. FEBS Lett 1994;345:237-240. [PubMed: 8200460]

1000. Packer MA, Murphy MP. Peroxynitrite formed by simultaneous nitric oxide and superoxide generation causes cyclosporin-A-sensitive mitochondrial calcium efflux and depolarisation. Eur J Biochem 1995;234:231-239. [PubMed: 8529645]

1001. Palacios-Callender M, Quintero M, Hollis VS, Springett RJ, Moncada S. Endogenous NO regulates superoxide production at low oxygen concentrations by modifying the redox state of cytochrome $c$ oxidase. Proc Natl Acad Sci USA 2004;101:7630-7635. [PubMed: 15136725]

1002. Pannala AS, Rice-Evans C, Sampson J, Singh S. Interaction of peroxynitrite with carotenoids and tocopherols within low density lipoprotein. FEBS Lett 1998;423:297-301. [PubMed: 9515727] 
1003. Pannu R, Singh I. Pharmacological strategies for the regulation of inducible nitric oxide synthase: neurodegenerative versus neuroprotective mechanisms. Neurochem Int 2006;49:170-182. [PubMed: 16765486]

1004. Paravicini TM, Touyz RM. Redox signaling in hypertension. Cardiovasc Res 2006;71:247-258. [PubMed: 16765337]

1005. Park S, Geddes TJ, Javitch JA, Kuhn DM. Dopamine prevents nitration of tyrosine hydroxylase by peroxynitrite and nitrogen dioxide: is nitrotyrosine formation an early step in dopamine neuronal damage? J Biol Chem 2003;278:28736-28742. [PubMed: 12771134]

1006. Park SW, Huq MD, Hu X, Wei LN. Tyrosine nitration on p65: a novel mechanism to rapidly inactivate nuclear factor-kappaB. Mol Cell Proteomics 2005;4:300-309. [PubMed: 15657065]

1007. Park SW, Wei LN. Regulation of c-myc gene by nitric oxide via inactivating NF-kappa B complex in P19 mouse embryonal carcinoma cells. J Biol Chem 2003;278:29776-29782. [PubMed: 12783888]

1008. Parmentier S, Bohme GA, Lerouet D, Damour D, Stutzmann JM, Margaill I, Plotkine M. Selective inhibition of inducible nitric oxide synthase prevents ischaemic brain injury. Br J Pharmacol 1999;127:546-552. [PubMed: 10385257]

1009. Patel RP, Darley-Usmar VM. Using peroxynitrite as oxidant with low-density lipoprotein. Methods Enzymol 1996;269:375-384. [PubMed: 8791666]

1010. Patel RP, Diczfalusy U, Dzeletovic S, Wilson MT, Darley-Usmar VM. Formation of oxysterols during oxidation of low density lipoprotein by peroxynitrite, myoglobin, copper. J Lipid Res 1996;37:2361-2371. [PubMed: 8978488]

1011. Patel RP, McAndrew J, Sellak H, White CR, Jo H, Freeman BA, Darley-Usmar VM. Biological aspects of reactive nitrogen species. Biochim Biophys Acta 1999;1411:385-400. [PubMed: 10320671]

1012. Patel RP, Moellering D, Murphy-Ullrich J, Jo H, Beckman JS, Darley-Usmar VM. Cell signaling by reactive nitrogen and oxygen species in atherosclerosis. Free Radic Biol Med 2000;28:17801794. [PubMed: 10946220]

1013. Pauling L. The discovery of the superoxide radical. Trends Biochem Sci 1979;4:N270-N271.

1014. Paulus WJ, Bronzwaer JG. Myocardial contractile effects of nitric oxide. Heart Fail Rev 2002;7:371-383. [PubMed: 12379822]

1015. Pearce LL, Kanai AJ, Birder LA, Pitt BR, Peterson J. The catabolic fate of nitric oxide: the nitric oxide oxidase and peroxynitrite reductase activities of cytochrome oxidase. J Biol Chem 2002;277:13556-13562. [PubMed: 11825895]

1016. Pearce LL, Kanai AJ, Epperly MW, Peterson J. Nitrosative stress results in irreversible inhibition of purified mitochondrial complexes I and III without modification of cofactors. Nitric Oxide 2005;13:254-263. [PubMed: 16185902]

1017. Pearce LL, Pitt BR, Peterson J. The peroxynitrite reductase activity of cytochrome $c$ oxidase involves a two-electron redox reaction at the heme a(3)-Cu(B) site. J Biol Chem 1999;274:3576335767. [PubMed: 10585458]

1018. Pehar M, Cassina P, Vargas MR, Castellanos R, Viera L, Beckman JS, Estevez AG, Barbeito L. Astrocytic production of nerve growth factor in motor neuron apoptosis: implications for amyotrophic lateral sclerosis. J Neurochem 2004;89:464-473. [PubMed: 15056289]

1019. Pennathur S, Bergt C, Shao B, Byun J, Kassim SY, Singh P, Green PS, McDonald TO, Brunzell J, Chait A, Oram JF, O’Brien K, Geary RL, Heinecke JW. Human atherosclerotic intima and blood of patients with established coronary artery disease contain high density lipoprotein damaged by reactive nitrogen species. J Biol Chem 2004;279:42977-42983. [PubMed: 15292228]

1020. Pennathur S, Jackson-Lewis V, Przedborski S, Heinecke JW. Mass spectrometric quantification of 3-nitrotyrosine, ortho-tyrosine, $o, o^{\prime}$-dityrosine in brain tissue of 1-methyl-4-phenyl-1,2,3, 6tetrahydropyridine-treated mice, a model of oxidative stress in Parkinson's disease. J Biol Chem 1999;274:34621-34628. [PubMed: 10574926]

1021. Pennathur S, Wagner JD, Leeuwenburgh C, Litwak KN, Heinecke JW. A hydroxyl radical-like species oxidizes cynomolgus monkey artery wall proteins in early diabetic vascular disease. J Clin Invest 2001;107:853-860. [PubMed: 11285304] 
1022. Perez-De La Cruz V, Gonzalez-Cortes C, Galvan-Arzate S, Medina-Campos ON, Perez-Severiano F, Ali SF, Pedraza-Chaverri J, Santamaria A. Excitotoxic brain damage involves early peroxynitrite formation in a model of Huntington's disease in rats: protective role of iron porphyrinate 5,10,15,20tetrakis (4-sulfonatophenyl)porphyrinate iron (III). Neuroscience 2005;135:463-474. [PubMed: 16111817]

1023. Perez-Severiano F, Escalante B, Vergara P, Rios C, Segovia J. Age-dependent changes in nitric oxide synthase activity and protein expression in striata of mice transgenic for the Huntington's disease mutation. Brain Res 2002;951:36-42. [PubMed: 12231454]

1024. Pesse B, Levrand S, Feihl F, Waeber B, Gavillet B, Pacher P, Liaudet L. Peroxynitrite activates ERK via Raf-1 and MEK, independently from EGF receptor and p21Ras in H9C2 cardiomyocytes. J Mol Cell Cardiol 2005;38:765-775. [PubMed: 15850570]

1025. Peters O, Back T, Lindauer U, Busch C, Megow D, Dreier J, Dirnagl U. Increased formation of reactive oxygen species after permanent and reversible middle cerebral artery occlusion in the rat. J Cereb Blood Flow Metab 1998;18:196-205. [PubMed: 9469163]

1026. Pick M, Rabani J, Yost F, Fridovich I. The catalytic mechanism of the manganese-containing superoxide dismutase of Escherichia coli studied by pulse radiolysis. J Am Chem Soc 1974;96:7329-7332. [PubMed: 4610039]

1027. Pieper GM, Nilakantan V, Chen M, Zhou J, Khanna AK, Henderson JD Jr, Johnson CP, Roza AM, Szabo C. Protective mechanisms of a metalloporphyrinic peroxynitrite decomposition catalyst, WW85, in rat cardiac transplants. J Pharmacol Exp Ther 2005;314:53-60. [PubMed: 15784653]

1028. Pierson KB, Evenson MA. $200 \mathrm{Kd}$ neurofilament protein binds Al, Cu and Zn. Biochem Biophys Res Commun 1988;152:598-604. [PubMed: 3130052]

1029. Pinlaor S, Ma N, Hiraku Y, Yongvanit P, Semba R, Oikawa S, Murata M, Sripa B, Sithithaworn $\mathrm{P}$, Kawanishi S. Repeated infection with Opisthorchis viverrini induces accumulation of 8nitroguanine and 8-oxo-7,8-dihydro-2'-deoxyguanine in the bile duct of hamsters via inducible nitric oxide synthase. Carcinogenesis 2004;25:1535-1542. [PubMed: 15059927]

1030. Piper HM, Garcia-Dorado D, Ovize M. A fresh look at reperfusion injury. Cardiovasc Res 1998;38:291-300. [PubMed: 9709390]

1031. Plumb RC, Tantayanon R, Libby M, Xu WW. Chemical model for Viking biology experiments: implications for the composition of the martian regolith. Nature 1989;338:633-635.

1032. Podgoreanu MV, Michelotti GA, Sato Y, Smith MP, Lin S, Morris RW, Grocott HP, Mathew JP, Schwinn DA. Differential cardiac gene expression during cardiopulmonary bypass: ischemiaindependent upregulation of proinflammatory genes. J Thorac Cardiovasc Surg 2005;130:330-339. [PubMed: 16077395]

1033. Podrez EA, Schmitt D, Hoff HF, Hazen SL. Myeloperoxidase-generated reactive nitrogen species convert LDL into an atherogenic form in vitro. J Clin Invest 1999;103:1547-1560. [PubMed: 10359564]

1034. Poladia DP, Bauer JA. Early cell-specific changes in nitric oxide synthases, reactive nitrogen species formation, ubiquitinylation during diabetes-related bladder remodeling. Diabetes Metab Res Rev 2003;19:313-319. [PubMed: 12879409]

1035. Pollock DM, Pollock JS. Endothelin and oxidative stress in the vascular system. Curr Vasc Pharmacol 2005;3:365-367. [PubMed: 16248780]

1036. Polman CH, Uitdehaag BM. Drug treatment of multiple sclerosis. BMJ 2000;321:490-494. [PubMed: 10948033]

1037. Pong K. Oxidative stress in neurodegenerative diseases: therapeutic implications for superoxide dismutase mimetics. Expert Opin Biol Ther 2003;3:127-139. [PubMed: 12718737]

1038. Potashkin JA, Meredith GE. The role of oxidative stress in the dysregulation of gene expression and protein metabolism in neurodegenerative disease. Antioxid Redox Signal 2006;8:144-151. [PubMed: 16487048]

1039. Pozza M, Bettelli C, Magnani F, Mascia MT, Manzini E, Calza L. Is neuronal nitric oxide involved in adjuvant-induced joint inflammation? Eur J Pharmacol 1998;359:87-93. [PubMed: 9831298]

1040. Pricci F, Leto G, Amadio L, Iacobini C, Cordone S, Catalano S, Zicari A, Sorcini M, Di Mario U, Pugliese G. Oxidative stress in diabetes-induced endothelial dysfunction involvement of nitric oxide and protein kinase C. Free Radic Biol Med 2003;35:683-694. [PubMed: 12957660] 
1041. Pryor WA, Squadrito GL. The chemistry of peroxynitrite: a product from the reaction of nitric oxide with superoxide. Am J Physiol Lung Cell Mol Physiol 1995;268:L699-L722.

1042. Przedborski S, Chen Q, Vila M, Giasson BI, Djaldatti R, Vukosavic S, Souza JM, Jackson-Lewis $\mathrm{V}$, Lee VM, Ischiropoulos H. Oxidative post-translational modifications of alpha-synuclein in the 1-methyl-4-phenyl-1,2,3,6-tetrahydropyridine (MPTP) mouse model of Parkinson's disease. J Neurochem 2001;76:637-640. [PubMed: 11208927]

1043. Przedborski S, Jackson-Lewis V, Djaldetti R, Liberatore G, Vila M, Vukosavic S, Almer G. The parkinsonian toxin MPTP: action and mechanism. Restor Neurol Neurosci 2000;16:135-142. [PubMed: 12671216]

1044. Przedborski S, Jackson-Lewis V, Yokoyama R, Shibata T, Dawson VL, Dawson TM. Role of neuronal nitric oxide in 1-methyl-4-phenyl-1,2,3,6-tetrahydropyridine (MPTP)-induced dopaminergic neurotoxicity. Proc Natl Acad Sci USA 1996;93:4565-4571. [PubMed: 8643444]

1045. Pu M, Akhand AA, Kato M, Hamaguchi M, Koike T, Iwata H, Sabe H, Suzuki H, Nakashima I. Evidence of a novel redox-linked activation mechanism for the Src kinase which is independent of tyrosine 527-mediated regulation. Oncogene 1996;13:2615-2622. [PubMed: 9000135]

1046. Pulido EJ, Shames BD, Selzman CH, Barton HA, Banerjee A, Bensard DD, McIntyre RC Jr. Inhibition of PARS attenuates endotoxin-induced dysfunction of pulmonary vasorelaxation. Am J Physiol Lung Cell Mol Physiol 1999;277:L769-L776.

1047. Qayyum I, Zubrow AB, Ashraf QM, Kubin J, Delivoria-Papadopoulos M, Mishra OP. Nitration as a mechanism of $\mathrm{Na}^{+}, \mathrm{K}^{+}$-ATPase modification during hypoxia in the cerebral cortex of the guinea pig fetus. Neurochem Res 2001;26:1163-1169. [PubMed: 11700960]

1048. Qi M, Elion EA. MAP kinase pathways. J Cell Sci 2005;118:3569-3572. [PubMed: 16105880]

1049. Quagliaro L, Piconi L, Assaloni R, Martinelli L, Motz E, Ceriello A. Intermittent high glucose enhances apoptosis related to oxidative stress in human umbilical vein endothelial cells: the role of protein kinase C and NAD(P)H-oxidase activation. Diabetes 2003;52:2795-2804. [PubMed: 14578299]

1050. Rabini RA, Vignini A, Salvolini E, Staffolani R, Martarelli D, Moretti N, Mazzanti L. Activation of human aortic endothelial cells by LDL from Type 1 diabetic patients: an in vitro study. Atherosclerosis 2002;165:69-77. [PubMed: 12208472]

1051. Rabinovitch A, Suarez-Pinzon WL, Sorensen O, Bleackley RC. Inducible nitric oxide synthase (iNOS) in pancreatic islets of nonobese diabetic mice: identification of iNOS-expressing cells and relationships to cytokines expressed in the islets. Endocrinology 1996;137:2093-2099. [PubMed: 8612552]

1052. Rachmilewitz D, Stamler JS, Karmeli F, Mullins ME, Singel DJ, Loscalzo J, Xavier RJ, Podolsky DK. Peroxynitrite-induced rat colitis: a new model of colonic inflammation. Gastroenterology 1993;105:1681-1688. [PubMed: 8253344]

1053. Radi R. Nitric oxide, oxidants, protein tyrosine nitration. Proc Natl Acad Sci USA 2004;101:40034008. [PubMed: 15020765]

1054. Radi R. Peroxynitrite reactions and diffusion in biology. Chem Res Toxicol 1998;11:720-721. [PubMed: 9671533]

1055. Radi R, Beckman JS, Bush KM, Freeman BA. Peroxynitrite-induced membrane lipid peroxidation: the cytotoxic potential of superoxide and nitric oxide. Arch Biochem Biophys 1991;288:481-487. [PubMed: 1654835]

1056. Radi R, Beckman JS, Bush KM, Freeman BA. Peroxynitrite-mediated sulfhydryl oxidation: the cytotoxic potential of superoxide and nitric oxide. J Biol Chem 1991;266:4244-4250. [PubMed: 1847917]

1057. Radi R, Beckman JS, Bush KM, Freeman BA. Peroxynitrite oxidation of sulfhydryls. The cytotoxic potential of superoxide and nitric oxide. J Biol Chem 1991;266:4244-4250. [PubMed: 1847917]

1058. Radi R, Cassina A, Hodara R. Nitric oxide and peroxynitrite interactions with mitochondria. Biol Chem 2002;383:401-409. [PubMed: 12033431]

1059. Radi R, Cassina A, Hodara R, Quijano C, Castro L. Peroxynitrite reactions and formation in mitochondria. Free Radic Biol Med 2002;33:1451-1464. [PubMed: 12446202]

1060. Radi R, Cosgrove TP, Beckman JS, Freeman BA. Peroxynitrite-induced luminol chemiluminescence. Biochem J 1993;290:51-57. [PubMed: 8382481] 
1061. Radi R, Peluffo G, Alvarez MN, Naviliat M, Cayota A. Unraveling peroxynitrite formation in biological systems. Free Radic Biol Med 2001;30:463-488. [PubMed: 11182518]

1062. Radi R, Rodriguez M, Castro L, Telleri R. Inhibition of mitochondrial electron transport by peroxynitrite. Arch Biochem Biophys 1994;308:89-95. [PubMed: 8311480]

1063. Radons J, Heller B, Burkle A, Hartmann B, Rodriguez ML, Kroncke KD, Burkart V, Kolb H. Nitric oxide toxicity in islet cells involves poly(ADP-ribose) polymerase activation and concomitant NAD ${ }^{+}$depletion. Biochem Biophys Res Commun 1994;199:1270-1277. [PubMed: 8147870]

1064. Raghuram N, Fortenberry JD, Owens ML, Brown LA. Effects of exogenous nitric oxide and hyperoxia on lung fibroblast viability and DNA fragmentation. Biochem Biophys Res Commun 1999;262:685-691. [PubMed: 10471386]

1065. Rajagopalan S, Kurz S, Munzel T, Tarpey M, Freeman BA, Griendling KK, Harrison DG. Angiotensin II-mediated hypertension in the rat increases vascular superoxide production via membrane NADH/NADPH oxidase activation. Contribution to alterations of vasomotor tone. J Clin Invest 1996;97:1916-1923. [PubMed: 8621776]

1066. Rajfer J, Aronson WJ, Bush PA, Dorey FJ, Ignarro LJ. Nitric oxide as a mediator of relaxation of the corpus cavernosum in response to nonadrenergic, noncholinergic neurotransmission. $\mathrm{N}$ Engl $\mathrm{J}$ Med 1992;326:90-94. [PubMed: 1309211]

1067. Rakesh K, Agrawal DK. Controlling cytokine signaling by constitutive inhibitors. Biochem Pharmacol 2005;70:649-657. [PubMed: 15936728]

1068. Raoul C, Estevez AG, Nishimune H, Cleveland DW, deLapeyriere O, Henderson CE, Haase G, Pettmann B. Motoneuron death triggered by a specific pathway downstream of Fas. Potentiation by ALS-linked SOD1 mutations. Neuron 2002;35:1067-1083. [PubMed: 12354397]

1069. Ravalli S, Albala A, Ming M, Szabolcs M, Barbone A, Michler RE, Cannon PJ. Inducible nitric oxide synthase expression in smooth muscle cells and macrophages of human transplant coronary artery disease. Circulation 1998;97:2338-2345. [PubMed: 9639378]

1070. Reaume AG, Elliott JL, Hoffman EK, Kowall NW, Ferrante RJ, Siwek DF, Wilcox HM, Flood DG, Beal MF, Brown RH Jr, Scott RW, Snider WD. Motor neurons in Cu/Zn superoxide dismutasedeficient mice develop normally but exhibit enhanced cell death after axonal injury. Nat Genet 1996;13:43-47. [PubMed: 8673102]

1071. Reid AB, Kurten RC, McCullough SS, Brock RW, Hinson JA. Mechanisms of acetaminopheninduced hepatotoxicity: role of oxidative stress and mitochondrial permeability transition in freshly isolated mouse hepatocytes. J Pharmacol Exp Ther 2005;312:509-516. [PubMed: 15466245]

1072. Reinehr R, Gorg B, Hongen A, Haussinger D. CD95-tyrosine nitration inhibits hyperosmotic and CD95 ligand-induced CD95 activation in rat hepatocytes. J Biol Chem 2004;279:10364-10373. [PubMed: 14679192]

1073. Reynolds MR, Berry RW, Binder LI. Site-specific nitration and oxidative dityrosine bridging of the tau protein by peroxynitrite: implications for Alzheimer's disease. Biochemistry 2005;44:16901700. [PubMed: 15683253]

1074. Reynolds MR, Lukas TJ, Berry RW, Binder LI. Peroxynitrite-mediated tau modifications stabilize preformed filaments and destabilize microtubules through distinct mechanisms. Biochemistry 2006;45:4314-4326. [PubMed: 16566606]

1075. Richter C. Biophysical consequences of lipid peroxidation in membranes. Chem Phys Lipids 1987;44:175-189. [PubMed: 3311416]

1076. Ridnour LA, Isenberg JS, Espey MG, Thomas DD, Roberts DD, Wink DA. Nitric oxide regulates angiogenesis through a functional switch involving thrombospondin-1. Proc Natl Acad Sci USA 2005;102:13147-13152. [PubMed: 16141331]

1077. Roberts CK, Vaziri ND, Ni Z, Barnard RJ. Correction of long-term diet-induced hypertension and nitrotyrosine accumulation by diet modification. Atherosclerosis 2002;163:321-327. [PubMed: 12052479]

1078. Robinson VK, Sato E, Nelson DK, Camhi SL, Robbins RA, Hoyt JC. Peroxynitrite inhibits inducible (type 2) nitric oxide synthase in murine lung epithelial cells in vitro. Free Radic Biol Med 2001;30:986-991. [PubMed: 11316578]

1079. Rodrigo J, Fernandez AP, Serrano J, Peinado MA, Martinez A. The role of free radicals in cerebral hypoxia and ischemia. Free Radic Biol Med 2005;39:26-50. [PubMed: 15925277] 
1080. Rodriguez-Iturbe B, Zhan CD, Quiroz Y, Sindhu RK, Vaziri ND. Antioxidant-rich diet relieves hypertension and reduces renal immune infiltration in spontaneously hypertensive rats. Hypertension 2003;41:341-346. [PubMed: 12574105]

1081. Rodriguez-Martinez MA, Alonso MJ, Redondo J, Salaices M, Marin J. Role of lipid peroxidation and the glutathione-dependent antioxidant system in the impairment of endothelium-dependent relaxations with age. Br J Pharmacol 1998;123:113-121. [PubMed: 9484861]

1082. Rojas J, Paya M, Dominguez JN, Ferrandiz ML. ttCH, a selective inhibitor of inducible nitric oxide synthase expression with antiarthritic properties. Eur J Pharmacol 2003;465:183-189. [PubMed: 12650848]

1083. Ronson RS, Nakamura M, Vinten-Johansen J. The cardiovascular effects and implications of peroxynitrite. Cardiovasc Res 1999;44:47-59. [PubMed: 10615389]

1084. Ronson RS, Thourani VH, Ma XL, Katzmark SL, Han D, Zhao ZQ, Nakamura M, Guyton RA, Vinten-Johansen J. Peroxynitrite, the breakdown product of nitric oxide, is beneficial in blood cardioplegia but injurious in crystalloid cardioplegia. Circulation 1999;100(Suppl II):II-384II-391. [PubMed: 10567334]

1085. Rork TH, Hadzimichalis NM, Kappil MA, Merrill GF. Acetaminophen attenuates peroxynitriteactivated matrix metalloproteinase-2-mediated troponin I cleavage in the isolated guinea pig myocardium. J Mol Cell Cardiol 2006;40:553-561. [PubMed: 16530785]

1086. Rosen DR, Siddique T, Patterson D, Figlewicz DA, Sapp P, Hentati A, Donaldson D, Goto J, O'Regan JP, Deng HX, Rahmani Z, Krizus A, McKenna-Yasek D, Cayabyab A, Gaston SM, Berger R, Tanzi RE, Halperin JJ, Herzfeldt B, Van den Bergh R, Hung WY, Bird T, Deng G, Mulder DW, Smyth C, Laing NG, Soriano E, Pericak-Vance MA, Haines J, Rouleau GA, Gusella JS, Horvitz $\mathrm{HR}$, Brown RH Jr. Mutations in $\mathrm{Cu} / \mathrm{Zn}$ superoxide dismutase gene are associated with familial amyotrophic lateral sclerosis. Nature 1993;362:59-62. [PubMed: 8446170]

1087. Roskoski R Jr. Src kinase regulation by phosphorylation and dephosphorylation. Biochem Biophys Res Commun 2005;331:1-14. [PubMed: 15845350]

1088. Rowland LP, Shneider NA. Amyotrophic lateral sclerosis. N Engl J Med 2001;344:1688-1700. [PubMed: 11386269]

1089. Rubanyi GM, Vanhoutte PM. Superoxide anions and hyperoxia inactivate endothelium-derived relaxing factor. Am J Physiol Heart Circ Physiol 1986;250:H822-H827.

1090. Rubbo H, Batthyany C, Radi R. Nitric oxide-oxygen radicals interactions in atherosclerosis. Biol Res 2000;33:167-175. [PubMed: 15693284]

1091. Rubbo H, Darley-Usmar V, Freeman BA. Nitric oxide regulation of tissue free radical injury. Chem Res Toxicol 1996;9:809-820. [PubMed: 8828915]

1092. Rubbo H, Denicola A, Radi R. Peroxynitrite inactivates thiol-containing enzymes of Trypanosoma cruzi energetic metabolism and inhibits cell respiration. Arch Biochem Biophys 1994;308:96-102. [PubMed: 8311481]

1093. Rubbo H, O’Donnell V. Nitric oxide, peroxynitrite and lipoxygenase in atherogenesis: mechanistic insights. Toxicology 2005;208:305-317. [PubMed: 15691594]

1094. Rubbo H, Trostchansky A, Botti H, Batthyany C. Interactions of nitric oxide and peroxynitrite with low-density lipoprotein. Biol Chem 2002;383:547-552. [PubMed: 12033442]

1095. Ryberg H, Soderling AS, Davidsson P, Blennow K, Caidahl K, Persson LI. Cerebrospinal fluid levels of free 3-nitrotyrosine are not elevated in the majority of patients with amyotrophic lateral sclerosis or Alzheimer's disease. Neurochem Int 2004;45:57-62. [PubMed: 15082222]

1096. Ryu JK, Kim SU, McLarnon JG. Blockade of quinolinic acid-induced neurotoxicity by pyruvate is associated with inhibition of glial activation in a model of Huntington's disease. Exp Neurol 2004;187:150-159. [PubMed: 15081596]

1097. Sabetkar M, Low SY, Naseem KM, Bruckdorfer KR. The nitration of proteins in platelets: significance in platelet function. Free Radic Biol Med 2002;33:728-736. [PubMed: 12208361]

1098. Sacksteder CA, Qian WJ, Knyushko TV, Wang H, Chin MH, Lacan G, Melega WP, Camp DG 2nd, Smith RD, Smith DJ, Squier TC, Bigelow DJ. Endogenously nitrated proteins in mouse brain: links to neurodegenerative disease. Biochemistry 2006;45:8009-8022. [PubMed: 16800626] 
1099. Saeki M, Kamisaki Y, Maeda S. Involvement of mitogen-activated protein kinase in peroxynitriteinduced cell death of human neuroblastoma SH-SY5Y cells. Neurosci Res 2000;38:213-216. [PubMed: 11000449]

1100. Saeki M, Maeda S. p130cas is a cellular target protein for tyrosine nitration induced by peroxynitrite. Neurosci Res 1999;33:325-328. [PubMed: 10401986]

1101. Saeki M, Maeda S, Kamisaki Y. Vanadate protects human neuroblastoma SH-SY5Y cells against peroxynitrite-induced cell death. J Cell Biochem 2002;85:721-727. [PubMed: 11968012]

1102. Saez TE, Pehar M, Vargas M, Barbeito L, Maccioni RB. Astrocytic nitric oxide triggers tau hyperphosphorylation in hippocampal neurons. In Vivo 2004;18:275-280. [PubMed: 15341183]

1103. Sakurai H, Kohsaka H, Liu MF, Higashiyama H, Hirata Y, Kanno K, Saito I, Miyasaka N. Nitric oxide production and inducible nitric oxide synthase expression in inflammatory arthritides. J Clin Invest 1995;96:2357-2363. [PubMed: 7593623]

1104. Sakurai M, Fukuyama N, Iguchi A, Akimoto H, Ohmi M, Yokoyama H, Nakazawa H, Tabayashi K. Quantitative analysis of cardiac 3-L-nitrotyrosine during acute allograft rejection in an experimental heart transplantation. Transplantation 1999;68:1818-1822. [PubMed: 10609964]

1105. Salgo MG, Squadrito GL, Pryor WA. Peroxynitrite causes apoptosis in rat thymocytes. Biochem Biophys Res Commun 1995;215:1111-1118. [PubMed: 7488038]

1106. Salom JB, Orti M, Centeno JM, Torregrosa G, Alborch E. Reduction of infarct size by the NO donors sodium nitroprusside and spermine/NO after transient focal cerebral ischemia in rats. Brain Res 2000;865:149-156. [PubMed: 10821916]

1107. Salvemini D, Cuzzocrea S. Oxidative stress in septic shock and disseminated intravascular coagulation. Free Radic Biol Med 2002;33:1173-1185. [PubMed: 12398925]

1108. Salvemini D, Mazzon E, Dugo L, Serraino I, De Sarro A, Caputi AP, Cuzzocrea S. Amelioration of joint disease in a rat model of collagen-induced arthritis by M40403, a superoxide dismutase mimetic. Arthritis Rheum 2001;44:2909-2921. [PubMed: 11762952]

1109. Salvemini D, Riley DP, Lennon PJ, Wang ZQ, Currie MG, Macarthur H, Misko TP. Protective effects of a superoxide dismutase mimetic and peroxynitrite decomposition catalysts in endotoxininduced intestinal damage. Br J Pharmacol 1999;127:685-692. [PubMed: 10401559]

1110. Salvemini D, Wang ZQ, Bourdon DM, Stern MK, Currie MG, Manning PT. Evidence of peroxynitrite involvement in the carrageenan-induced rat paw edema. Eur J Pharmacol 1996;303:217-220. [PubMed: 8813572]

1111. Salvemini D, Wang ZQ, Stern MK, Currie MG, Misko TP. Peroxynitrite decomposition catalysts: therapeutics for peroxynitrite-mediated pathology. Proc Natl Acad Sci USA 1998;95:2659-2663. [PubMed: 9482943]

1112. Sampson JB, Rosen H, Beckman JS. Peroxynitrite-dependent tyrosine nitration catalyzed by superoxide dismutase, myeloperoxidase, horseradish peroxidase. Methods Enzymol 1996;269:210-218. [PubMed: 8791651]

1113. Sampson JB, Ye Y, Rosen H, Beckman JS. Myeloperoxidase and horseradish peroxidase catalyze tyrosine nitration in proteins from nitrite and hydrogen peroxide. Arch Biochem Biophys 1998;356:207-213. [PubMed: 9705211]

1114. Sanchez-Ramos JR, Hefti F, Weiner WJ. Paraquat and Parkinson's disease. Neurology 1987;37:728. [PubMed: 3561790]

1115. Sandhu JK, Robertson S, Birnboim HC, Goldstein R. Distribution of protein nitrotyrosine in synovial tissues of patients with rheumatoid arthritis and osteoarthritis. J Rheumatol 2003;30:11731181. [PubMed: 12784386]

1116. Sangha O. Epidemiology of rheumatic diseases. Rheumatology 2000;39(Suppl 2):3-12. [PubMed: 11276800]

1117. Santiago-Lopez D, Vazquez-Roman B, Perez-de La Cruz V, Barrera D, Rembao D, Salinas-Lara C, Pedraza-Chaverri J, Galvan-Arzate S, Ali SF, Santamaria A. Peroxynitrite decomposition catalyst, iron metalloporphyrin, reduces quinolinate-induced neurotoxicity in rats. Synapse 2004;54:233-238. [PubMed: 15484207]

1118. Saraswathi V, Wu G, Toborek M, Hennig B. Linoleic acid-induced endothelial activation: role of calcium and peroxynitrite signaling. J Lipid Res 2004;45:794-804. [PubMed: 14993245] 
1119. Sarchielli P, Galli F, Floridi A, Floridi A, Gallai V. Relevance of protein nitration in brain injury: a key pathophysiological mechanism in neurodegenerative, autoimmune, or inflammatory CNS diseases and stroke. Amino Acids 2003;25:427-436. [PubMed: 14661102]

1120. Sarker KP, Nakata M, Kitajima I, Nakajima T, Maruyama I. Inhibition of caspase-3 activation by SB 203580, p38 mitogen-activated protein kinase inhibitor in nitric oxide-induced apoptosis of PC-12 cells. J Mol Neurosci 2000;15:243-250. [PubMed: 11303787]

1121. Sasaki S, Warita H, Abe K, Iwata M. Inducible nitric oxide synthase (iNOS) and nitrotyrosine immunoreactivity in the spinal cords of transgenic mice with a G93A mutant SOD1 gene. J Neuropathol Exp Neurol 2001;60:839-846. [PubMed: 11556540]

1122. Sase K, Michel T. Expression and regulation of endothelial nitric oxide synthase. Trends Cardiovasc Med 1997;7:28-37.

1123. Satsangi J, Silverberg MS, Vermeire S, Colombel JF. The Montreal classification of inflammatory bowel disease: controversies, consensus, implications. Gut 2006;55:749-753. [PubMed: 16698746]

1124. Sawa T, Ohshima H. Nitrative DNA damage in inflammation and its possible role in carcinogenesis. Nitric Oxide 2006;14:91-100. [PubMed: 16099698]

1125. Sawyer DT, Valentine J. How super is superoxide? Acct Chem Res 1981;14:393-400.

1126. Saxena M, Mustelin T. Extracellular signals and scores of phosphatases: all roads lead to MAP kinase. Semin Immunol 2000;12:387-396. [PubMed: 10995585]

1127. Scarlett JL, Packer MA, Porteous CM, Murphy MP. Alterations to glutathione and nicotinamide nucleotides during the mitochondrial permeability transition induced by peroxynitriten. Biochem Pharmacol 1996;52:1047-1055. [PubMed: 8831724]

1128. Schieke SM, Briviba K, Klotz LO, Sies H. Activation pattern of mitogen-activated protein kinases elicited by peroxynitrite: attenuation by selenite supplementation. FEBS Lett 1999;448:301-303. [PubMed: 10218497]

1129. Schlessinger J. Cell signaling by receptor tyrosine kinases. Cell 2000;103:211-225. [PubMed: 11057895]

1130. Schmidt P, Youhnovski N, Daiber A, Balan A, Arsic M, Bachschmid M, Przybylski M, Ullrich V. Specific nitration at tyrosine 430 revealed by high resolution mass spectrometry as basis for redox regulation of bovine prostacyclin synthase. J Biol Chem 2003;278:12813-12819. [PubMed: 12562775]

1131. Schoneich C, Viner RI, Ferrington DA, Bigelow DJ. Age-related chemical modification of the skeletal muscle sarcoplasmic reticulum Ca-ATPase of the rat. Mech Ageing Dev 1999;107:221231. [PubMed: 10360678]

1132. Schopfer FJ, Baker PR, Freeman BA. NO-dependent protein nitration: a cell signaling event or an oxidative inflammatory response? Trends Biochem Sci 2003;28:646-654. [PubMed: 14659696]

1133. Schroeder P, Klotz LO, Buchczyk DP, Sadik CD, Schewe T, Sies H. Epicatechin selectively prevents nitration but not oxidation reactions of peroxynitrite. Biochem Biophys Res Commun 2001;285:782-787. [PubMed: 11453660]

1134. Schroeder P, Zhang H, Klotz LO, Kalyanaraman B, Sies H. (-)-Epicatechin inhibits nitration and dimerization of tyrosine in hydrophilic as well as hydrophobic environments. Biochem Biophys Res Commun 2001;289:1334-1338. [PubMed: 11741342]

1135. Schulz JB, Matthews RT, Klockgether T, Dichgans J, Beal MF. The role of mitochondrial dysfunction and neuronal nitric oxide in animal models of neurodegenerative diseases. Mol Cell Biochem 1997;174:193-197. [PubMed: 9309687]

1136. Schulz R, Dodge KL, Lopaschuk GD, Clanachan AS. Peroxynitrite impairs cardiac contractile function by decreasing cardiac efficiency. Am J Physiol Heart Circ Physiol 1997;272:H1212H1219.

1137. Schuman EM, Madison DV. A requirement for the intercellular messenger nitric oxide in longterm potentiation. Science 1991;254:1503-1506. [PubMed: 1720572]

1138. Schutte H, Mayer K, Burger H, Witzenrath M, Gessler T, Seeger W, Grimminger F. Endogenous nitric oxide synthesis and vascular leakage in ischemic-reperfused rabbit lungs. Am J Respir Crit Care Med 2001;164:412-418. [PubMed: 11500342] 
1139. Schutte H, Witzenrath M, Mayer K, Rosseau S, Seeger W, Grimminger F. Short-term "preconditioning" with inhaled nitric oxide protects rabbit lungs against ischemia-reperfusion injury. Transplantation 2001;72:1363-1370. [PubMed: 11685104]

1140. Scott DJ, Hull MA, Cartwright EJ, Lam WK, Tisbury A, Poulsom R, Markham AF, Bonifer C, Coletta PL. Lack of inducible nitric oxide synthase promotes intestinal tumorigenesis in the Apc (Min/+) mouse. Gastroenterology 2001;121:889-899. [PubMed: 11606502]

1141. Scott GS, Hooper DC. The role of uric acid in protection against peroxynitrite-mediated pathology. Med Hypotheses 2001;56:95-100. [PubMed: 11133262]

1142. Scott GS, Kean RB, Southan GJ, Szabo C, Hooper DC. Effect of mercaptoethylguanidine scavengers of peroxynitrite on the development of experimental allergic encephalomyelitis in PLSJL mice. Neurosci Lett 2001;311:125-128. [PubMed: 11567794]

1143. Sekar K. Inhaled nitric oxide in term and preterm infants. J Perinatol 2006;26(Suppl 1):S4-S23. [PubMed: 16625225]

1144. Seki J, Nishio M, Kato Y, Motoyama Y, Yoshida K. FK409, a new nitricoxide donor, suppresses smooth muscle proliferation in the rat model of balloon angioplasty. Atherosclerosis 1995;117:97106. [PubMed: 8546759]

1145. Senftleben U, Cao Y, Xiao G, Greten FR, Krahn G, Bonizzi G, Chen Y, Hu Y, Fong A, Sun SC, Karin M. Activation by IKKalpha of a second, evolutionary conserved, NF-kappa B signaling pathway. Science 2001;293:1495-1499. [PubMed: 11520989]

1146. Serracino-Inglott F, Virlos IT, Habib NA, Williamson RC, Mathie RT. Differential nitric oxide synthase expression during hepatic ischemia-reperfusion. Am J Surg 2003;185:589-595. [PubMed: 12781892]

1147. Serrano F, Kolluri NS, Wientjes FB, Card JP, Klann E. NADPH oxidase immunoreactivity in the mouse brain. Brain Res 2003;988:193-198. [PubMed: 14519542]

1148. Shacka JJ, Sahawneh MA, Gonzalez JD, Ye YZ, D’Alessandro TL, Estevez AG. Two distinct signaling pathways regulate peroxynitrite-induced apoptosis in PC12 cells. Cell Death Differ 2006;13:1506-1514. [PubMed: 16410804]

1149. Shah AM, MacCarthy PA. Paracrine and autocrine effects of nitric oxide on myocardial function. Pharmacol Ther 2000;86:49-86. [PubMed: 10760546]

1150. Shall S, de Murcia G. Poly(ADP-ribose) polymerase-1: what have we learned from the deficient mouse model? Mutat Res 2000;460:1-15. [PubMed: 10856830]

1151. Sharma VS, Traylor TG, Gardiner R, Mizukami H. Reaction of nitric oxide with heme proteins and model compounds of hemoglobin. Biochemistry 1987;26:3837-3843. [PubMed: 3651417]

1152. Shears LL 2nd, Kibbe MR, Murdock AD, Billiar TR, Lizonova A, Kovesdi I, Watkins SC, Tzeng E. Efficient inhibition of intimal hyperplasia by adenovirus-mediated inducible nitric oxide synthase gene transfer to rats and pigs in vivo. J Am Coll Surg 1998;187:295-306. [PubMed: 9740187]

1153. Sheng H, Enghild JJ, Bowler R, Patel M, Batinic-Haberle I, Calvi CL, Day BJ, Pearlstein RD, Crapo JD, Warner DS. Effects of metalloporphyrin catalytic antioxidants in experimental brain ischemia. Free Radic Biol Med 2002;33:947-961. [PubMed: 12361805]

1154. Sherman MP, Griscavage JM, Ignarro LJ. Nitric oxide-mediated neuronal injury in multiple sclerosis. Med Hypotheses 1992;39:143-146. [PubMed: 1461176]

1155. Shi H, Noguchi N, Xu Y, Niki E. Formation of phospholipid hydroperoxides and its inhibition by alpha-tocopherol in rat brain synaptosomes induced by peroxynitrite. Biochem Biophys Res Commun 1999;257:651-656. [PubMed: 10208838]

1156. Shibata M, Araki N, Hamada J, Sasaki T, Shimazu K, Fukuu-chi Y. Brain nitrite production during global ischemia and reperfusion: an in vivo microdialysis study. Brain Res 1996;734:86-90. [PubMed: 8896812]

1157. Shibuya A, Wada K, Nakajima A, Saeki M, Katayama K, Mayumi T, Kadowaki T, Niwa H, Kamisaki Y. Nitration of PPARgamma inhibits ligand-dependent translocation into the nucleus in a macrophage-like cell line, RAW 264. FEBS Lett 2002;525:43-47. [PubMed: 12163159]

1158. Shimizu K, Rajapakse N, Horiguchi T, Payne RM, Busija DW. Neuroprotection against hypoxiaischemia in neonatal rat brain by novel superoxide dismutase mimetics. Neurosci Lett 2003;346:4144. [PubMed: 12850543] 
1159. Shimosawa T, Shibagaki Y, Ishibashi K, Kitamura K, Kangawa K, Kato S, Ando K, Fujita T. Adrenomedullin, an endogenous peptide, counteracts cardiovascular damage. Circulation 2002;105:106-111. [PubMed: 11772884]

1160. Shin T, Tanuma N, Kim S, Jin J, Moon C, Kim K, Kohyama K, Matsumoto Y, Hyun B. An inhibitor of inducible nitric oxide synthase ameliorates experimental autoimmune myocarditis in Lewis rats. J Neuroimmunol 1998;92:133-138. [PubMed: 9916888]

1161. Shiva S, Brookes PS, Patel RP, Anderson PG, Darley-Usmar VM. Nitric oxide partitioning into mitochondrial membranes and the control of respiration at cytochrome $c$ oxidase. Proc Natl Acad Sci USA 2001;98:7212-7217. [PubMed: 11416204]

1162. Shrivastava P, Pantano C, Watkin R, McElhinney B, Guala A, Poynter ML, Persinger RL, Budd $\mathrm{R}$, Janssen-Heininger Y. Reactive nitrogen species-induced cell death requires Fas-dependent activation of c-Jun N-terminal kinase. Mol Cell Biol 2004;24:6763-6772. [PubMed: 15254243]

1163. Siegert A, Rosenberg C, Schmitt WD, Denkert C, Hauptmann S. Nitric oxide of human colorectal adenocarcinoma cell lines promotes tumour cell invasion. Br J Cancer 2002;86:1310-1315. [PubMed: 11953890]

1164. Silvagno F, Xia H, Bredt DS. Neuronal nitricoxide synthase- $\mu$, an alternatively spliced isoform expressed in differentiated skeletal muscle. J Biol Chem 1996;271:11204-11208. [PubMed: 8626668]

1165. Simbulan-Rosenthal CM, Ly DH, Rosenthal DS, Konopka G, Luo R, Wang ZQ, Schultz PG, Smulson E. Misregulation of gene expression in primary fibroblasts lacking poly(ADP-ribose) polymerase. Proc Natl Acad Sci USA 2000;97:11274-11279. [PubMed: 11016956]

1166. Simic G, Lucassen PJ, Krsnik Z, Kruslin B, Kostovic I, Winblad B, Bogdanovi. nNOS expression in reactive astrocytes correlates with increased cell death related DNA damage in the hippocampus and entorhinal cortex in Alzheimer's disease. Exp Neurol 2000;165:12-26. [PubMed: 10964481]

1167. Simonian NA, Coyle JT. Oxidative stress in neurodegenerative diseases. Annu Rev Pharmacol Toxicol 1996;36:83-106. [PubMed: 8725383]

1168. Simpson EP. Antioxidant treatment for amyotrophic lateral sclerosis. Lancet Neurol 2005;4:266. [PubMed: 15847835]

1169. Sims NR, Anderson MF. Mitochondrial contributions to tissue damage in stroke. Neurochem Int 2002;40:511-526. [PubMed: 11850108]

1170. Singer II, Kawka DW, Scott S, Weidner JR, Mumford RA, Riehl TE, Stenson WF. Expression of inducible nitric oxide synthase and nitrotyrosine in colonic epithelium in inflammatory bowel disease. Gastroenterology 1996;111:871-885. [PubMed: 8831582]

1171. Singh IN, Sullivan PG, Deng Y, Mbye LH, Hall ED. Time course of post-traumatic mitochondrial oxidative damage and dysfunction in a mouse model of focal traumatic brain injury: implications for neuroprotective therapy. J Cereb Blood Flow Metab. In press

1172. Sinnaeve P, Chiche JD, Nong Z, Varenne O, Van Pelt N, Gillijns H, Collen D, Bloch KD, Janssens S. Soluble guanylate cyclase alpha(1) and beta(1) gene transfer increases NO responsiveness and reduces neointima formation after balloon injury in rats via antiproliferative and antimigratory effects. Circ Res 2001;88:103-109. [PubMed: 11139481]

1173. Sironi L, Cimino M, Guerrini U, Calvio AM, Lodetti B, Asdente M, Balduini W, Paoletti R, Tremoli E. Treatment with statins after induction of focal ischemia in rats reduces the extent of brain damage. Arterioscler Thromb Vasc Biol 2003;23:322-327. [PubMed: 12588778]

1174. Smith CD, Carson M, van der Woerd M, Chen J, Ischiropoulos H, Beckman JS. Crystal structure of peroxynitrite-modified bovine $\mathrm{Cu}, \mathrm{Zn}$ superoxide dismutase. Arch Biochem Biophys 1992;299:350-355. [PubMed: 1444476]

1175. Smith KJ, Kapoor R, Felts PA. Demyelination: the role of reactive oxygen and nitrogen species. Brain Pathol 1999;9:69-92. [PubMed: 9989453]

1176. Smith MA, Richey Harris PL, Sayre LM, Beckman JS, Perry G. Widespread peroxynitrite-mediated damage in Alzheimer's disease. J Neurosci 1997;17:2653-2657. [PubMed: 9092586]

1177. Sobel BE, Schneider DJ. Cardiovascular complications in diabetes mellitus. Curr Opin Pharmacol 2005;5:143-148. [PubMed: 15780822] 
1178. Sohn HY, Krotz F, Zahler S, Gloe T, Keller M, Theisen K, Schiele TM, Klauss V, Pohl U. Crucial role of local peroxynitrite formation in neutrophil-induced endothelial cell activation. Cardiovasc Res 2003;57:804-815. [PubMed: 12618242]

1179. Soloviev A, Stefanov A, Parshikov A, Khromov A, Moibenko A, Kvotchina L, Balavoine G, Geletii Y. Arrhythmogenic peroxynitrite-induced alterations in mammalian heart contractility and its prevention with quercetin-filled liposomes. Cardiovasc Toxicol 2002;2:129-139. [PubMed: 12271156]

1180. Somers MJ, Mavromatis K, Galis ZS, Harrison DG. Vascular superoxide production and vasomotor function in hypertension induced by deoxycorticosterone acetate-salt. Circulation 2000;101:17221728. [PubMed: 10758056]

1181. Sood HS, Cox MJ, Tyagi SC. Generation of nitrotyrosine precedes activation of metalloproteinase in myocardium of hyperhomocysteinemic rats. Antioxid Redox Signal 2002;4:799-804. [PubMed: 12470508]

1182. Sorescu D, Griendling KK. Reactive oxygen species, mitochondria, NAD $(\mathrm{P}) \mathrm{H}$ oxidases in the development and progression of heart failure. Congest Heart Fail 2002;8:132-140. [PubMed: 12045381]

1183. Soriano FG, Liaudet L, Szabo E, Virag L, Mabley JG, Pacher P, Szabo C. Resistance to acute septic peritonitis in poly(ADP-ribose) polymerase-1-deficient mice. Shock 2002;17:286-292. [PubMed: 11954828]

1184. Soriano FG, Nogueira AC, Caldini EG, Lins MH, Teixeira AC, Cappi SB, Lotufo PA, Bernik MM, Zsengeller Z, Chen M, Szabo C. Potential role of poly(adenosine 5'-diphosphate-ribose) polymerase activation in the pathogenesis of myocardial contractile dysfunction associated with human septic shock. Crit Care Med 2006;34:1073-1079. [PubMed: 16484919]

1185. Soriano FG, Pacher P, Mabley J, Liaudet L, Szabo C. Rapid reversal of the diabetic endothelial dysfunction by pharmacological inhibition of poly(ADP-ribose) polymerase. Circ Res 2001;89:684-691. [PubMed: 11597991]

1186. Soriano FG, Virag L, Szabo C. Diabetic endothelial dysfunction: role of reactive oxygen and nitrogen species production and poly-(ADP-ribose) polymerase activation. J Mol Med 2001;79:437-448. [PubMed: 11511974]

1187. Sospedra M, Martin R. Immunology of multiple sclerosis. Annu Rev Immunol 2005;23:683-747. [PubMed: 15771584]

1188. Southam E, East SJ, Garthwaite J. Excitatory amino acid receptors coupled to the nitric oxide/ cyclic GMP pathway in rat cerebellum during development. J Neurochem 1991;56:2072-2081. [PubMed: 1673999]

1189. Southern C, Schulster D, Green IC. Inhibition of insulin secretion from rat islets of Langerhans by interleukin-6. An effect distinct from that of interleukin-1. Biochem J 1990;272:243-245. [PubMed: 2264829]

1190. Souza JM, Daikhin E, Yudkoff M, Raman CS, Ischiropoulos H. Factors determining the selectivity of protein tyrosine nitration. Arch Biochem Biophys 1999;371:169-178. [PubMed: 10545203]

1191. Souza JM, Giasson BI, Chen Q, Lee VM, Ischiropoulos H. Dityrosine cross-linking promotes formation of stable alpha-synuclein polymers. Implication of nitrative and oxidative stress in the pathogenesis of neurodegenerative synucleinopathies. J Biol Chem 2000;275:18344-18349. [PubMed: 10747881]

1192. Souza JM, Radi R. Glyceraldehyde-3-phosphate dehydrogenase inactivation by peroxynitrite. Arch Biochem Biophys 1998;360:187-194. [PubMed: 9851830]

1193. Spear N, Estevez AG, Barbeito L, Beckman JS, Johnson GV. Nerve growth factor protects PC12 cells against peroxynitrite-induced apoptosis via a mechanism dependent on phosphatidylinositol 3-kinase. J Neurochem 1997;69:53-59. [PubMed: 9202293]

1194. Spitaler MM, Graier WF. Vascular targets of redox signalling in diabetes mellitus. Diabetologia 2002;45:476-494. [PubMed: 12032623]

1195. Spitsin S, Hooper DC, Mikheeva T, Koprowski H. Uric acid levels in patients with multiple sclerosis: analysis in mono- and dizygotic twins. Mult Scler 2001;7:165-166. [PubMed: 11475439] 
1196. St Clair EW, Wilkinson WE, Lang T, Sanders L, Misukonis MA, Gilkeson GS, Pisetsky DS, Granger DL, Weinberg JB. Increased expression of blood mononuclear cell nitric oxide synthase type 2 in rheumatoid arthritis patients. J Exp Med 1996;184:1173-1178. [PubMed: 9064335]

1197. Stachowiak O, Dolder M, Wallimann T, Richter C. Mitochondrial creatine kinase is a prime target of peroxynitrite-induced modification and inactivation. J Biol Chem 1998;273:16694-16699. [PubMed: 9642223]

1198. Stadler K, Jenei V, von Bolcshazy G, Somogyi A, Jakus J. Increased nitric oxide levels as an early sign of premature aging in diabetes. Free Radic Biol Med 2003;35:1240-1251. [PubMed: 14607523]

1199. Stadtman ER, Moskovitz J, Levine RL. Oxidation of methionine residues of proteins: biological consequences. Antioxid Redox Signal 2003;5:577-582. [PubMed: 14580313]

1200. Stamler JS, Lamas S, Fang FC. Nitrosylation, the prototypic redox-based signaling mechanism. Cell 2001;106:675-683. [PubMed: 11572774]

1201. Stamler JS, Singel DJ, Loscalzo J. Biochemistry of nitric oxide and its redox-activated forms. Science 1992;258:1898-1902. [PubMed: 1281928]

1202. Stanger O, Weger M. Interactions of homocysteine, nitric oxide, folate and radicals in the progressively damaged endothelium. Clin Chem Lab Med 2003;41:1444-1454. [PubMed: 14656024]

1203. Stefanovic-Racic M, Meyers K, Meschter C, Coffey JW, Hoffman RA, Evans CH. Comparison of the nitric oxide synthase inhibitors methylarginine and aminoguanidine as prophylactic and therapeutic agents in rat adjuvant arthritis. J Rheumatol 1995;22:1922-1928. [PubMed: 8991992]

1204. Stevens MJ. Oxidative-nitrosative stress as a contributing factor to cardiovascular disease in subjects with diabetes. Curr Vasc Pharmacol 2005;3:253-266. [PubMed: 16026322]

1205. Stewart VC, Heales SJ. Nitric oxide-induced mitochondrial dysfunction: implications for neurodegeneration. Free Radic Biol Med 2003;34:287-303. [PubMed: 12543245]

1206. Stoker AW. Protein tyrosine phosphatases and signalling. J Endocrinol 2005;185:19-33. [PubMed: 15817824]

1207. Strong JP, Malcom GT, McMahan CA, Tracy RE, Newman WP 3rd, Herderick EE, Cornhill JF. Prevalence and extent of atherosclerosis in adolescents and young adults: implications for prevention from the Pathobiological Determinants of Atherosclerosis in Youth Study. JAMA 1999;281:727-735. [PubMed: 10052443]

1208. Stuehr DJ, Nathan CF. Nitric oxide. A macrophage product responsible for cytostasis and respiratory inhibition in tumor cells. J Exp Med 1989;169:1543-1555. [PubMed: 2497225]

1209. Suarez-Pinzon WL, Mabley JG, Strynadka K, Power RF, Szabo C, Rabinovitch A. An inhibitor of inducible nitric oxide synthase and scavenger of peroxynitrite prevents diabetes development in NOD mice. J Autoimmun 2001;16:449-455. [PubMed: 11437493]

1210. Suarez-Pinzon WL, Szabo C, Rabinovitch A. Development of autoimmune diabetes in NOD mice is associated with the formation of peroxynitrite in pancreatic islet beta-cells. Diabetes 1997;46:907-911. [PubMed: 9133563]

1211. Sucu N, Unlu A, Tamer L, Aytacoglu B, Ercan B, Dikmengil M, Atik U. 3-Nitrotyrosine in atherosclerotic blood vessels. Clin Chem Lab Med 2003;41:23-25. [PubMed: 12636045]

1212. Suh JH, Shigeno ET, Morrow JD, Cox B, Rocha AE, Frei B, Hagen TM. Oxidative stress in the aging rat heart is reversed by dietary supplementation with $(R)$-(alpha)-lipoic acid. FASEB $\mathrm{J}$ 2001;15:700-706. [PubMed: 11259388]

1213. Sun D, Huang A, Yan EH, Wu Z, Yan C, Kaminski PM, Oury TD, Wolin MS, Kaley G. Reduced release of nitric oxide to shear stress in mesenteric arteries of aged rats. Am J Physiol Heart Circ Physiol 2004;286:H2249-H2256. [PubMed: 14751861]

1214. Sun Y, Zhang J, Lu L, Chen SS, Quinn MT, Weber KT. Aldosterone-induced inflammation in the rat heart: role of oxidative stress. Am J Pathol 2002;161:1773-1781. [PubMed: 12414524]

1215. Suschek C, Fehsel K, Kroncke KD, Sommer A, Kolb-Bachofen V. Primary cultures of rat islet capillary endothelial cells. Constitutive and cytokine-inducible macrophagelike nitric oxide synthases are expressed and activities regulated by glucose concentration. Am J Pathol 1994;145:685-695. [PubMed: 7521579] 
1216. Susin SA, Lorenzo HK, Zamzami N, Marzo I, Snow BE, Brothers GM, Mangion J, Jacotot E, Costantini P, Loeffler M, Larochette N, Goodlett DR, Aebersold R, Siderovski DP, Penninger JM, Kroemer G. Molecular characterization of mitochondrial apoptosis-inducing factor. Nature 1999;397:441-446. [PubMed: 9989411]

1217. Suzuki A, Yamamoto N, Jokura H, Yamamoto M, Fujii A, Tokimitsu I, Saito I. Chlorogenic acid attenuates hypertension and improves endothelial function in spontaneously hypertensive rats. J Hypertens 2006;24:1065-1073. [PubMed: 16685206]

1218. Suzuki M, Tabuchi M, Ikeda M, Tomita T. Concurrent formation of peroxynitrite with the expression of inducible nitric oxide synthase in the brain during middle cerebral artery occlusion and reperfusion in rats. Brain Res 2002;951:113-120. [PubMed: 12231464]

1219. Suzuki N, Yasui M, Geacintov NE, Shafirovich V, Shibutani S. Miscoding events during DNA synthesis past the nitration-damaged base 8-nitroguanine. Biochemistry 2005;44:9238-9245. [PubMed: 15966748]

1220. Suzuki Y, Deitch EA, Mishima S, Lu Q, Xu D. Inducible nitric oxide synthase gene knockout mice have increased resistance to gut injury and bacterial translocation after an intestinal ischemiareperfusion injury. Crit Care Med 2000;28:3692-3696. [PubMed: 11098975]

1221. Suzuki YJ, Forman HJ, Sevanian A. Oxidants as stimulators of signal transduction. Free Radic Biol Med 1997;22:269-285. [PubMed: 8958153]

1222. Symmons D, Turner G, Webb R, Asten P, Barrett E, Lunt M, Scott D, Silman A. The prevalence of rheumatoid arthritis in the United Kingdom: new estimates for a new century. Rheumatology 2002;41:793-800. [PubMed: 12096230]

1223. Szabo A, Hake P, Salzman AL, Szabo C. 3-Aminobenzamide, an inhibitor of poly (ADP-ribose) synthetase, improves hemodynamics and prolongs survival in a porcine model of hemorrhagic shock. Shock 1998;10:347-353. [PubMed: 9840650]

1224. Szabo A, Hake P, Salzman AL, Szabo C. Beneficial effects of mercaptoethylguanidine, an inhibitor of the inducible isoform of nitric oxide synthase and a scavenger of peroxynitrite, in a porcine model of delayed hemorrhagic shock. Crit Care Med 1999;27:1343-1350. [PubMed: 10446830]

1225. Szabo A, Salzman AL, Szabo C. Poly(ADP-ribose) synthetase activation mediates pulmonary microvascular and intestinal mucosal dysfunction in endotoxin shock. Life Sci 1998;63:2133-2139. [PubMed: 9839537]

1226. Szabo C. Alterations in nitric oxide production in various forms of circulatory shock. New Horizons 1995;3:2-32. [PubMed: 7535648]

1227. Szabo C. Multiple pathways of peroxynitrite cytotoxicity. Toxicol Lett 2003;140-141:105-112.

1228. Szabo C. Pharmacological inhibition of poly(ADP-ribose) polymerase in cardiovascular disorders: future directions. Curr Vasc Pharmacol 2005;3:301-303. [PubMed: 16026326]

1229. Szabo C. Physiological and pathophysiological roles of nitric oxide in the central nervous system. Brain Res Bull 1996;41:131-141. [PubMed: 8886382]

1230. Szabo C. Poly(ADP-ribose) polymerase activation by reactive nitrogen species-Relevance for the pathogenesis of inflammation. Nitric Oxide 2006;14:169-179. [PubMed: 16111903]

1231. Szabo C. Potential role of the peroxynitrate-poly(ADP-ribose) synthetase pathway in a rat model of severe hemorrhagic shock. Shock 1998;9:341-344. [PubMed: 9617883]

1232. Szabo C. Roles of poly(ADP-ribose) polymerase activation in the pathogenesis of diabetes mellitus and its complications. Pharmacol Res 2005;52:60-71. [PubMed: 15911334]

1233. Szabo C, Cuzzocrea S, Zingarelli B, O’Connor M, Salzman AL. Endothelial dysfunction in a rat model of endotoxic shock. Importance of the activation of poly (ADP-ribose) synthetase by peroxynitrite. J Clin Invest 1997;100:723-735. [PubMed: 9239421]

1234. Szabo C, Mabley JG, Moeller SM, Shimanovich R, Pacher P, Virag L, Soriano FG, Van Duzer JH, Williams W, Salzman AL, Groves JT. Part I: pathogenetic role of peroxynitrite in the development of diabetes and diabetic vascular complications: studies with FP15, a novel potent peroxynitrite decomposition catalyst. Mol Med 2002;8:571-580. [PubMed: 12477967]

1235. Szabo C, Ohshima H. DNA damage induced by peroxynitrite: subsequent biological effects. Nitric Oxide 1997;1:373-385. [PubMed: 9441908] 
1236. Szabo C, Pacher P, Zsengeller Z, Vaslin A, Komjati K, Benko R, Chen M, Mabley JG, Kollai M. Angiotensin II-mediated endothelial dysfunction: role of poly(ADP-ribose) polymerase activation. Mol Med 2004;10:28-35. [PubMed: 15502880]

1237. Szabo C, Salzman AL, Ischiropoulos H. Endotoxin triggers the expression of an inducible isoform of nitric oxide synthase and the formation of peroxynitrite in the rat aorta in vivo. FEBS Lett 1995;363:235-238. [PubMed: 7537701]

1238. Szabo C, Salzman AL, Ischiropoulos H. Peroxynitrite-mediated oxidation of dihydrorhodamine 123 occurs in early stages of endotoxic and hemorrhagic shock and ischemia-reperfusion injury. FEBS Lett 1995;372:229-232. [PubMed: 7556674]

1239. Szabo C, Saunders C, O'Connor M, Salzman AL. Peroxynitrite causes energy depletion and increases permeability via activation of poly(ADP-ribose) synthetase in pulmonary epithelial cells. Am J Respir Cell Mol Biol 1997;16:105-109. [PubMed: 9032115]

1240. Szabo C, Virag L, Cuzzocrea S, Scott GJ, Hake P, O’Connor MP, Zingarelli B, Salzman AL, Kun E. Protection against peroxynitrite-induced fibroblast injury and arthritis development by inhibition of poly(ADP-ribose) synthetase. Proc Natl Acad Sci USA 1998;95:3867-3872. [PubMed: 9520459]

1241. Szabo C, Virag L, Cuzzocrea S, Scott GS, Hake P, O’Connor MP, Zingarelli B, Salzman A, Kun E. Protection against peroxynitrite-induced fibroblast injury and arthritis development by inhibition of poly(ADP-ribose) synthase. Proc Natl Acad Sci USA 1998;95:3867-3872. [PubMed: 9520459]

1242. Szabo C, Zanchi A, Komjati K, Pacher P, Krolewski AS, Quist WC, LoGerfo FW, Horton ES, Veves A. Poly(ADP-ribose) polymerase is activated in subjects at risk of developing type 2 diabetes and is associated with impaired vascular reactivity. Circulation 2002;106:2680-2686. [PubMed: 12438293]

1243. Szabo C, Zingarelli B, O'Connor M, Salzman AL. DNA strand breakage, activation of poly (ADPribose) synthetase, cellular energy depletion are involved in the cytotoxicity of macrophages and smooth muscle cells exposed to peroxynitrite. Proc Natl Acad Sci USA 1996;93:1753-1758. [PubMed: 8700830]

1244. Szabo C, Zingarelli B, Salzman AL. Role of poly-ADP ribosyl-transferase activation in the vascular contractile and energetic failure elicited by exogenous and endogenous nitric oxide and peroxynitrite. Circ Res 1996;78:1051-1063. [PubMed: 8635236]

1245. Szabo G, Bahrle S. Role of nitrosative stress and poly(ADP-ribose) polymerase activation in myocardial reperfusion injury. Curr Vasc Pharmacol 2005;3:215-220. [PubMed: 16026318]

1246. Szabo G, Liaudet L, Hagl S, Szabo C. Poly(ADP-ribose) polymerase activation in the reperfused myocardium. Cardiovasc Res 2004;61:471-480. [PubMed: 14962478]

1247. Szabolcs M, Michler RE, Yang X, Aji W, Roy D, Athan E, Sciacca RR, Minanov OP, Cannon PJ. Apoptosis of cardiac myocytes during cardiac allograft rejection. Relation to induction of nitric oxide synthase. Circulation 1996;94:1665-1673. [PubMed: 8840859]

1248. Szabolcs MJ, Ma N, Athan E, Zhong J, Ming M, Sciacca RR, Husemann J, Albala A, Cannon PJ. Acute cardiac allograft rejection in nitric oxide synthase-2(-/-) and nitric oxide synthase-2(+/+) mice: effects of cellular chimeras on myocardial inflammation and cardiomyocyte damage and apoptosis. Circulation 2001;103:2514-2520. [PubMed: 11369694]

1249. Szabolcs MJ, Ravalli S, Minanov O, Sciacca RR, Michler RE, Cannon PJ. Apoptosis and increased expression of inducible nitric oxide synthase in human allograft rejection. Transplantation 1998;65:804-812. [PubMed: 9539092]

1250. Szilvassy Z, Csont T, Pali T, Droy-Lefaix MT, Ferdinandy P. Nitric oxide, peroxynitrite and cGMP in atherosclerosis-induced hypertension in rabbits: beneficial effects of cicletanine. J Vasc Res 2001;38:39-46. [PubMed: 11173993]

1251. Tabner BJ, El-Agnaf OM, German MJ, Fullwood NJ, Allsop D. Protein aggregation, metals and oxidative stress in neurodegenerative diseases. Biochem Soc Trans 2005;33:1082-1086. [PubMed: 16246050]

1252. Tabuchi M, Umegaki K, Ito T, Suzuki M, Tomita I, Ikeda M, Tomita T. Fluctuation of serum NO (x) concentration at stroke onset in a rat spontaneous stroke model (M-SHRSP). Peroxynitrite formation in brain lesions. Brain Res 2002;949:147-156. [PubMed: 12213310] 
1253. Taddei S, Virdis A, Mattei P, Ghiadoni L, Gennari A, Fasolo CB, Sudano I, Salvetti A. Aging and endothelial function in normotensive subjects and patients with essential hypertension. Circulation 1995;91:1981-1987. [PubMed: 7895356]

1254. Takakura K, Beckman JS, MacMillan-Crow LA, Crow JP. Rapid and irreversible inactivation of protein tyrosine phosphatases PTP1B, CD45, LAR by peroxynitrite. Arch Biochem Biophys 1999;369:197-207. [PubMed: 10486138]

1255. Takakura K, Xiaohong W, Takeuchi K, Fukuda S. Peroxynitrite decreases dopamine's vasoconstrictive activity. Anesth Analg 2003;97:1492-1496. [PubMed: 14570672]

1256. Takakura K, Xiaohong W, Takeuchi K, Yasuda Y, Fukuda S. Deactivation of norepinephrine by peroxynitrite as a new pathogenesis in the hypotension of septic shock. Anesthesiology 2003;98:928-934. [PubMed: 12657855]

1257. Takamatsu Y, Shimada K, Yamaguchi K, Kuroki S, Chijiiwa K, Tanaka M. Inhibition of inducible nitric oxide synthase prevents hepatic, but not pulmonary, injury following ischemia-reperfusion of rat liver. Dig Dis Sci 2006;51:571-579. [PubMed: 16614969]

1258. Takizawa S, Aratani Y, Fukuyama N, Maeda N, Hirabayashi H, Koyama H, Shinohara Y, Nakazawa H. Deficiency of myeloperoxidase increases infarct volume and nitrotyrosine formation in mouse brain. J Cereb Blood Flow Metab 2002;22:50-54. [PubMed: 11807393]

1259. Takizawa S, Fukuyama N, Hirabayashi H, Nakazawa H, Shinohara Y. Dynamics of nitrotyrosine formation and decay in rat brain during focal ischemia-reperfusion. J Cereb Blood Flow Metab 1999;19:667-672. [PubMed: 10366197]

1260. Takizawa S, Hirabayashi H, Fukuyama N, Nakazawa H, Shinohara Y. Peroxynitrite production in cerebral ischemia. Rinsho Shinkeigaku 1999;39:1295-1297. [PubMed: 10791101]

1261. Tan S, Yokoyama Y, Dickens E, Cash TG, Freeman BA, Parks DA. Xanthine oxidase activity in the circulation of rats following hemorrhagic shock. Free Radic Biol Med 1993;15:407-414. [PubMed: 8225022]

1262. Tanabe T, Maeda S, Miyauchi T, Iemitsu M, Takanashi M, Irukayama-Tomobe Y, Yokota T, Ohmori H, Matsuda M. Exercise training improves ageing-induced decrease in eNOS expression of the aorta. Acta Physiol Scand 2003;178:3-10. [PubMed: 12713509]

1263. Taner AS, Cinel I, Ozer L, Onde U, Taner D, Koksoy C. Poly(ADP-ribose) synthetase inhibition reduces bacterial translocation in rats after endotoxin challenge. Shock 2001;16:159-162. [PubMed: 11508870]

1264. Tannous M, Cheung R, Vignini A, Mutus B. Atorvastatin increases ecNOS levels in human platelets of hyperlipidemic subjects. Thromb Haemost 1999;82:1390-1394. [PubMed: 10595624]

1265. Tannous M, Rabini RA, Vignini A, Moretti N, Fumelli P, Zielinski B, Mazzanti L, Mutus B. Evidence for iNOS-dependent peroxynitrite production in diabetic platelets. Diabetologia 1999;42:539-544. [PubMed: 10333045]

1266. Tauskela JS, Brunette E, O’Reilly N, Mealing G, Comas T, Gendron TF, Monette R, Morley P. An alternative $\mathrm{Ca}^{2+}$-dependent mechanism of neuroprotection by the metalloporphyrin class of superoxide dismutase mimetics. FASEB J 2005;19:1734-1736. [PubMed: 16081500]

1267. Taylor EL, Rossi AG, Shaw CA, Dal Rio FP, Haslett C, Megson IL. GEA 3162 decomposes to cogenerate nitric oxide and superoxide and induces apoptosis in human neutrophils via a peroxynitritedependent mechanism. Br J Pharmacol 2004;143:179-185. [PubMed: 15289284]

1268. Tecder-Unal M, Kanzyk Y. Peroxynitrite in reperfusion arrhythmias and its whole blood chemiluminescence results. Pharmacol Res 2004;49:7-16. [PubMed: 14597146]

1269. Tedeschi G, Cappelletti G, Negri A, Pagliato L, Maggioni MG, Maci R, Ronchi S. Characterization of nitroproteome in neuron-like PC12 cells differentiated with nerve growth factor: identification of two nitration sites in alpha-tubulin. Proteomics 2005;5:2422-2432. [PubMed: 15887183]

1270. Tedgui A, Mallat Z. Anti-inflammatory mechanisms in the vascular wall. Circ Res 2001;88:877887. [PubMed: 11348996]

1271. Teng RJ, Ye YZ, Parks DA, Beckman JS. Urate produced during hypoxia protects heart proteins from peroxynitrite-mediated protein nitration. Free Radic Biol Med 2002;33:1243-1249. [PubMed: 12398932] 
1272. Thatcher GR, Nicolescu AC, Bennett BM, Toader V. Nitrates and NO release: contemporary aspects in biological and medicinal chemistry. Free Radic Biol Med 2004;37:1122-1143. [PubMed: 15451053]

1273. Thiyagarajan M, Kaul CL, Sharma SS. Neuroprotective efficacy and therapeutic time window of peroxynitrite decomposition catalysts in focal cerebral ischemia in rats. Br J Pharmacol 2004;142:899-911. [PubMed: 15197101]

1274. Thomas SM, Brugge JS. Cellular functions regulated by Src family kinases. Annu Rev Cell Dev Biol 1997;13:513-609. [PubMed: 9442882]

1275. Thomson L, Trujillo M, Telleri R, Radi R. Kinetics of cytochrome $c$ oxidation by peroxynitrite: implications for superoxide measurements in nitric oxide-producing biological systems. Arch Biochem Biophys 1995;319:491-497. [PubMed: 7786032]

1276. Thorns V, Hansen L, Masliah E. nNOS expressing neurons in the entorhinal cortex and hippocampus are affected in patients with Alzheimer's disease. Exp Neurol 1998;150:14-20. [PubMed: 9514829]

1277. Thuraisingham RC, Nott CA, Dodd SM, Yaqoob MM. Increased nitrotyrosine staining in kidneys from patients with diabetic nephropathy. Kidney Int 2000;57:1968-1972. [PubMed: 10792615]

1278. Togo T, Katsuse O, Iseki E. Nitric oxide pathways in Alzheimer's disease and other neurodegenerative dementias. Neurol Res 2004;26:563-566. [PubMed: 15265275]

1279. Tohgi H, Abe T, Yamazaki K, Murata T, Ishizaki E, Isobe C. Alterations of 3-nitrotyrosine concentration in the cerebrospinal fluid during aging and in patients with Alzheimer's disease. Neurosci Lett 1999;269:52-54. [PubMed: 10821643]

1280. Tohgi H, Abe T, Yamazaki K, Murata T, Ishizaki E, Isobe C. Remarkable increase in cerebrospinal fluid 3-nitrotyrosine in patients with sporadic amyotrophic lateral sclerosis. Ann Neurol 1999;46:129-131. [PubMed: 10401792]

1281. Tokime T, Nozaki K, Sugino T, Kikuchi H, Hashimoto N, Ueda K. Enhanced poly(ADP-ribosyl) ation after focal ischemia in rat brain. J Cereb Blood Flow Metab 1998;18:991-997. [PubMed: 9740102]

1282. Tominaga T, Sato S, Ohnishi T, Ohnishi ST. Electron paramagnetic resonance (EPR) detection of nitric oxide produced during forebrain ischemia of the rat. J Cereb Blood Flow Metab 1994;14:715722. [PubMed: 8063867]

1283. Tonks NK. Redox redux: revisiting PTPs and the control of cell signaling. Cell 2005;121:667-670. [PubMed: 15935753]

1284. Torreilles F, Salman-Tabcheh S, Guerin M, Torreilles J. Neurodegenerative disorders: the role of peroxynitrite. Brain Res 1999;30:153-163.

1285. Tran MH, Yamada K, Nakajima A, Mizuno M, He J, Kamei H, Nabeshima T. Tyrosine nitration of a synaptic protein synaptophysin contributes to amyloid beta-peptide-induced cholinergic dysfunction. Mol Psychiatry 2003;8:407-412. [PubMed: 12740598]

1286. Traylor TG, Sharma VS. Why NO? Biochemistry 1992;31:2847-2849. [PubMed: 1348002]

1287. Trostchansky A, Ferrer-Sueta G, Batthyany C, Botti H, Batinic-Haberle I, Radi R, Rubbo H. Peroxynitrite flux-mediated LDL oxidation is inhibited by manganese porphyrins in the presence of uric acid. Free Radic Biol Med 2003;35:1293-1300. [PubMed: 14607528]

1288. Trostchansky A, Lind S, Hodara R, Oe T, Blair IA, Ischiropoulos H, Rubbo H, Souza JM. Interaction with phospholipids modulates alpha-synuclein nitration and lipid-protein adduct formation. Biochem J 2006;393:343-349. [PubMed: 16146428]

1289. Trujillo M, Folkes L, Bartesaghi S, Kalyanaraman B, Wardman P, Radi R. Peroxynitrite-derived carbonate and nitrogen dioxide radicals readily react with lipoic and dihydrolipoic acid. Free Radic Biol Med 2005;39:279-288. [PubMed: 15964519]

1290. Tsai HH, Hamilton TP, Tsai JHM, Beckman JS. Ab initio studies of peroxynitrite anion-water complexes. Struct Chem 1995;6:323-332.

1291. Tsai JHM, Harrison JG, Martin JC, Hamilton TP, van der Woerd M, Jablonsky MJ, Beckman JS. Role of conformation of peroxynitrite anion $\left(\mathrm{ONOO}^{-}\right)$in its stability and toxicity. $\mathrm{J}$ Am Chem Soc 1994;116:4115-4116. 
1292. Tsao PS, Aoki N, Lefer DJ, Johnson G 3rd, Lefer AM. Time course of endothelial dysfunction and myocardial injury during myocardial ischemia and reperfusion in the cat. Circulation 1990;82:1402-1412. [PubMed: 2401073]

1293. Tsoukias NM, Kavdia M, Popel AS. A theoretical model of nitric oxide transport in arterioles: frequency- vs. amplitude-dependent control of cGMP formation. Am J Physiol Heart Circ Physiol 2004;286:H1043-H1056. [PubMed: 14592938]

1294. Tsoukias NM, Popel AS. Erythrocyte consumption of nitric oxide in presence and absence of plasma-based hemoglobin. Am J Physiol Heart Circ Physiol 2002;282:H2265-H2277. [PubMed: 12003837]

1295. Tsuchihashi S, Kaldas F, Chida N, Sudo Y, Tamura K, Zhai Y, Qiao B, Busuttil RW, KupiecWeglinski JW. FK330, a novel inducible nitric oxide synthase inhibitor, prevents ischemia and reperfusion injury in rat liver transplantation. Am J Transplant. In press

1296. Tsukada K, Hasegawa T, Tsutsumi S, Katoh H, Kuwano H, Miyazaki T, Yamamoto Y. Effect of uric acid on liver injury during hemorrhagic shock. Surgery 2000;127:439-446. [PubMed: 10776436]

1297. Turan N, Csonka C, Csont T, Giricz Z, Fodor G, Bencsik P, Gyongyosi M, Cakici I, Ferdinandy $\mathrm{P}$. The role of peroxynitrite in chemical preconditioning with 3-nitropropionic acid in rat hearts. Cardiovasc Res. In press

1298. Turko IV, Li L, Aulak KS, Stuehr DJ, Chang JY, Murad F. Protein tyrosine nitration in the mitochondria from diabetic mouse heart. Implications to dysfunctional mitochondria in diabetes. $\mathbf{J}$ Biol Chem 2003;278:33972-33977. [PubMed: 12821649]

1299. Turko IV, Marcondes S, Murad F. Diabetes-associated nitration of tyrosine and inactivation of succinyl-CoA:3-oxoacid CoA-transferase. Am J Physiol Heart Circ Physiol 2001;281:H2289H2294. [PubMed: 11709394]

1300. Turko IV, Murad F. Protein nitration in cardiovascular diseases. Pharmacol Rev 2002;54:619-634. [PubMed: 12429871]

1301. Uc A, Kooy NW, Conklin JL, Bishop WP. Peroxynitrite inhibits epidermal growth factor receptor signaling in Caco-2 cells. Dig Dis Sci 2003;48:2353-2359. [PubMed: 14714624]

1302. Uchigata Y, Yamamoto H, Kawamura A, Okamoto H. Protection by superoxide dismutase, catalase, poly(ADP-ribose) synthetase inhibitors against alloxan- and streptozotocin-induced islet DNA strand breaks and against the inhibition of proinsulin synthesis. J Biol Chem 1982;257:60846088. [PubMed: 6281256]

1303. Ungvari Z, Csiszar A, Bagi Z, Koller A. Impaired nitric oxide-mediated flow-induced coronary dilation in hyperhomocysteinemia: morphological and functional evidence for increased peroxynitrite formation. Am J Pathol 2002;161:145-153. [PubMed: 12107099]

1304. Ungvari Z, Csiszar A, Huang A, Kaminski PM, Wolin MS, Koller A. High pressure induces superoxide production in isolated arteries via protein kinase $\mathrm{C}$-dependent activation of $\mathrm{NAD}(\mathrm{P}) \mathrm{H}$ oxidase. Circulation 2003;108:1253-1258. [PubMed: 12874194]

1305. Ungvari Z, Csiszar A, Kaminski PM, Wolin MS, Koller A. Chronic high pressure-induced arterial oxidative stress: involvement of protein kinase C-dependent NAD(P)H oxidase and local reninangiotensin system. Am J Pathol 2004;165:219-226. [PubMed: 15215177]

1306. Ungvari Z, Gupte SA, Recchia FA, Batkai S, Pacher P. Role of oxidative-nitrosative stress and downstream pathways in various forms of cardiomyopathy and heart failure. Curr Vasc Pharmacol 2005;3:221-229. [PubMed: 16026319]

1307. Upmacis RK, Deeb RS, Resnick MJ, Lindenbaum R, Gamss C, Mittar D, Hajjar DP. Involvement of the mitogen-activated protein kinase cascade in peroxynitrite-mediated arachidonic acid release in vascular smooth muscle cells. Am J Physiol Cell Physiol 2004;286:C1271-C1280. [PubMed: 14749211]

1308. Valdez LB, Zaobornyj T, Alvarez S, Bustamante J, Costa LE, Boveris A. Heart mitochondrial nitric oxide synthase. Effects of hypoxia and aging. Mol Aspects Med 2004;25:49-59. [PubMed: 15051316]

1309. Valentine JS, Doucette PA, Potter SZ. Copper-zinc superoxide dismutase and amyotrophic lateral sclerosis. Annu Rev Biochem 2005;74:563-593. [PubMed: 15952898] 
1310. Vallet P, Charnay Y, Steger K, Ogier-Denis E, Kovari E, Herrmann F, Michel JP, Szanto I. Neuronal expression of the NADPH oxidase NOX4, its regulation in mouse experimental brain ischemia. Neuroscience 2005;132:233-238. [PubMed: 15802177]

1311. Van der Loo B, Labugger R, Skepper JN, Bachschmid M, Kilo J, Powell JM, Palacios-Callender M, Erusalimsky JD, Quaschning T, Malinski T, Gygi D, Ullrich V, Luscher TF. Enhanced peroxynitrite formation is associated with vascular aging. J Exp Med 2000;192:1731-1744. [PubMed: 11120770]

1312. Van der Veen RC, Hinton DR, Incardonna F, Hofman FM. Extensive peroxynitrite activity during progressive stages of central nervous system inflammation. J Neuroimmunol 1997;77:1-7. [PubMed: 9209262]

1313. Van der Veen RC, Roberts LJ. Contrasting roles for nitric oxide and peroxynitrite in the peroxidation of myelin lipids. J Neuro-immunol 1999;95:1-7.

1314. Van der Vliet A, Eiserich JP, Halliwell B, Cross CE. Formation of reactive nitrogen species during peroxidase-catalyzed oxidation of nitrite. A potential additional mechanism of nitric oxidedependent toxicity. J Biol Chem 1997;272:7617-7625. [PubMed: 9065416]

1315. Van der Vliet A, Eiserich JP, Shigenaga MK, Cross CE. Reactive nitrogen species and tyrosine nitration in the respiratory tract: epiphenomena or a pathobiologic mechanism of disease? Am J Respir Crit Care Med 1999;160:1-9. [PubMed: 10390372]

1316. Van der Vliet A, Hristova M, Cross CE, Eiserich JP, Goldkorn T. Peroxynitrite induces covalent dimerization of epidermal growth factor receptors in A431 epidermoid carcinoma cells. J Biol Chem 1998;273:31860-31866. [PubMed: 9822654]

1317. Vanderheyden M, Bartunek J, Knaapen M, Kockx M, De Bruyne B, Goethals M. Hemodynamic effects of inducible nitric oxide synthase and nitrotyrosine generation in heart failure. J Heart Lung Transplant 2004;23:723-728. [PubMed: 15366433]

1318. Vanhoutte PM. Endothelial dysfunction in hypertension. J Hypertens Suppl 1996;14:S83-S93. [PubMed: 9120689]

1319. Varadarajan R, Golden-Mason L, Young L, McLoughlin P, Nolan N, McEntee G, Traynor O, Geoghegan J, Hegarty JE, O'Farrelly C. Nitric oxide in early ischaemia reperfusion injury during human orthotopic liver transplantation. Transplantation 2004;78:250-256. [PubMed: 15280686]

1320. Varela M, Herrera M, Garvin JL. Inhibition of Na-K-ATPase in thick ascending limbs by NO depends on $\mathrm{O}_{2}{ }^{-}$and is diminished by a high-salt diet. Am J Physiol Renal Physiol 2004;287:F224F230. [PubMed: 15113751]

1321. Vargas MR, Pehar M, Cassina P, Beckman JS, Barbeito L. Increased glutathione biosynthesis by Nrf2 activation in astrocytes prevents p75NTR-dependent motor neuron apoptosis. J Neurochem 2006;97:687-696. [PubMed: 16524372]

1322. Vargas MR, Pehar M, Cassina P, Estevez AG, Beckman JS, Barbeito L. Stimulation of nerve growth factor expression in astrocytes by peroxynitrite. In Vivo 2004;18:269-274. [PubMed: 15341182]

1323. Vaziri ND, Liang K, Ding Y. Increased nitric oxide inactivation by reactive oxygen species in leadinduced hypertension. Kidney Int 1999;56:1492-1498. [PubMed: 10504500]

1324. Vaziri ND, Ni Z. Expression of NOX-I, gp91phox, p47phox and P67phox in the aorta segments above and below coarctation. Biochim Biophys Acta 2005;1723:321-327. [PubMed: 15814300]

1325. Vaziri ND, Ni Z, Oveisi F, Liang K, Pandian R. Enhanced nitric oxide inactivation and protein nitration by reactive oxygen species in renal insufficiency. Hypertension 2002;39:135-141. [PubMed: 11799092]

1326. Vieira HL, Belzacq AS, Haouzi D, Bernassola F, Cohen I, Jacotot E, Ferri KF, El Hamel C, Bartle LM, Melino G, Brenner C, Goldmacher V, Kroemer G. The adenine nucleotide translocator: a target of nitric oxide, peroxynitrite, 4-hydroxynonenal. Oncogene 2001;20:4305-4316. [PubMed: 11466611]

1327. Viera, L.; Ye, Y.; Estévez, A.; Beckman, J. Immunohistochemical methods to detect nitrotyrosine. In: Packer, L., editor. Nitric Oxide: Biological and Antioxidant Activities. San Diego, CA: Academic; 1998. p. 373-381.

1328. Villa LM, Salas E, Darley-Usmar VM, Radomski MW, Moncada S. Peroxynitrite induces both vasodilatation and impaired vascular relaxation in the isolated perfused rat heart. Proc Natl Acad Sci USA 1994;91:12383-12387. [PubMed: 7809045] 
1329. Viner RI, Huhmer AF, Bigelow DJ, Schoneich C. The oxidative inactivation of sarcoplasmic reticulum Ca(2+)-ATPase by peroxynitrite. Free Radic Res 1996;24:243-259. [PubMed: 8731009]

1330. Viner RI, Williams TD, Schoneich C. Peroxynitrite modification of protein thiols: oxidation, nitrosylation, $S$-glutathiolation of functionally important cysteine residue(s) in the sarcoplasmic reticulum Ca-ATPase. Biochemistry 1999;38:12408-12415. [PubMed: 10493809]

1331. Virag L. Structure and function of poly(ADP-ribose) polymerase-1: role in oxidative stress-related pathologies. Curr Vasc Pharmacol 2005;3:209-214. [PubMed: 16026317]

1332. Virag L, Szabo C. BCL-2 protects peroxynitrite-treated thymocytes from poly(ADP-ribose) synthase (PARS)-independent apoptotic but not from PARS-mediated necrotic cell death. Free Radic Biol Med 2000;29:704-713. [PubMed: 11053771]

1333. Virag L, Szabo C. The therapeutic potential of poly(ADP-ribose) polymerase inhibitors. Pharmacol Rev 2002;54:375-429. [PubMed: 12223530]

1334. Virag L, Szabo E, Gergely P, Szabo C. Peroxynitrite-induced cytotoxicity: mechanism and opportunities for intervention. Toxicol Lett 2003;140-141:113-124.

1335. Virdis A, Neves MF, Amiri F, Viel E, Touyz RM, Schiffrin EL. Spironolactone improves angiotensin-induced vascular changes and oxidative stress. Hypertension 2002;40:504-510. [PubMed: 12364354]

1336. Von der Leyen HE, Gibbons GH, Morishita R, Lewis NP, Zhang L, Nakajima M, Kaneda Y, Cooke JP, Dzau VJ. Gene therapy inhibiting neointimal vascular lesion: in vivo transfer of endothelial cell nitric oxide synthase gene. Proc Natl Acad Sci USA 1995;92:1137-1141. [PubMed: 7532305]

1337. Walder CE, Green SP, Darbonne WC, Mathias J, Rae J, Dinauer MC, Curnutte JT, Thomas GR. Ischemic stroke injury is reduced in mice lacking a functional NADPH oxidase. Stroke 1997;28:2252-2258. [PubMed: 9368573]

1338. Waldow T, Alexiou K, Witt W, Wagner FM, Kappert U, Knaut M, Matschke K. Protection of lung tissue against ischemia/reper-fusion injury by preconditioning with inhaled nitric oxide in an in situ pig model of normothermic pulmonary ischemia. Nitric Oxide 2004;10:195-201. [PubMed: 15275865]

1339. Walford GA, Moussignac RL, Scribner AW, Loscalzo J, Leopold JA. Hypoxia potentiates nitric oxide-mediated apoptosis in endothelial cells via peroxynitrite-induced activation of mitochondriadependent and -independent pathways. J Biol Chem 2004;279:4425-4432. [PubMed: 14597620]

1340. Wallace MN, Geddes JG, Farquhar DA, Masson MR. Nitric oxide synthase in reactive astrocytes adjacent to beta-amyloid plaques. Exp Neurol 1997;144:266-272. [PubMed: 9168828]

1341. Wang HC, Zhang HF, Guo WY, Su H, Zhang KR, Li QX, Yan W, Ma XL, Lopez BL, Christopher TA, Gao F. Hypoxic postconditioning enhances the survival and inhibits apoptosis of cardiomyocytes following reoxygenation: role of peroxynitrite formation. Apoptosis 2006;11:1453-1460. [PubMed: 16761110]

1342. Wang HD, Xu S, Johns DG, Du Y, Quinn MT, Cayatte AJ, Cohen RA. Role of NADPH oxidase in the vascular hypertrophic and oxidative stress response to angiotensin II in mice. Circ Res 2001;88:947-953. [PubMed: 11349005]

1343. Wang JS, Singh H, Zhang F, Ishizuka T, Deng H, Kemp R, Wolin MS, Hintze TH, Abraham NG, Nasjletti A, Laniado-Schwartzman M. Endothelial dysfunction and hypertension in rats transduced with CYP4A2 adenovirus. Circ Res 2006;98:962-969. [PubMed: 16543501]

1344. Wang P, Zweier JL. Measurement of nitric oxide and peroxynitrite generation in the postischemic heart. Evidence for peroxynitrite-mediated reperfusion injury. J Biol Chem 1996;271:2922329230. [PubMed: 8910581]

1345. Wang W, Sawicki G, Schulz R. Peroxynitrite-induced myocardial injury is mediated through matrix metalloproteinase-2. Cardiovasc Res 2002;53:165-174. [PubMed: 11744025]

1346. Wang X, Feuerstein GZ. Role of immune and inflammatory mediators in CNS injury. Drug News Perspect 2000;13:133-140. [PubMed: 12937603]

1347. Wang Y, Lawson JA, Jaeschke H. Differential effect of 2-aminoethyl-isothiourea, an inhibitor of the inducible nitric oxide synthase, on microvascular blood flow and organ injury in models of hepatic ischemia-reperfusion and endotoxemia. Shock 1998;10:20-25. [PubMed: 9688086] 
1348. Watanabe H, Muramatsu Y, Kurosaki R, Michimata M, Matsubara M, Imai Y, Araki T. Protective effects of neuronal nitric oxide synthase inhibitor in mouse brain against MPTP neurotoxicity: an immunohistological study. Eur Neuropsychopharmacol 2004;14:93-104. [PubMed: 15013024]

1349. Wattanapitayakul SK, Weinstein DM, Holycross BJ, Bauer JA. Endothelial dysfunction and peroxynitrite formation are early events in angiotensin-induced cardiovascular disorders. FASEB J 2000;14:271-278. [PubMed: 10657983]

1350. Watts JA, Grattan RM, Whitlow BS, Kline JA. Activation of poly(ADP-ribose) polymerase in severe hemorrhagic shock and resuscitation. Am J Physiol Gastrointest Liver Physiol 2001;281:G498-G506. [PubMed: 11447030]

1351. Weber M, Lauer N, Mulsch A, Kojda G. The effect of peroxynitrite on the catalytic activity of soluble guanylyl cyclase. Free Radic Biol Med 2001;31:1360-1367. [PubMed: 11728807]

1352. Wei XQ, Charles IG, Smith A, Ure J, Feng GJ, Huang FP, Xu D, Muller W, Moncada S, Liew FY. Altered immune responses in mice lacking inducible nitric oxide synthase. Nature 1995;375:408411. [PubMed: 7539113]

1353. Weinberg JB. Nitric oxide production and nitric oxide synthase type 2 expression by human mononuclear phagocytes: a review. Mol Med 1998;4:557-591. [PubMed: 9848075]

1354. Weinberg JB, Granger DL, Pisetsky DS, Seldin MF, Misukonis MA, Mason SN, Pippen AM, Ruiz P, Wood ER, Gilkeson GS. The role of nitric oxide in the pathogenesis of spontaneous murine autoimmune disease: increased nitric oxide production and nitric oxide synthase expression in MRL-lpr/lpr mice, reduction of spontaneous glomerulonephritis and arthritis by orally administered $N^{\mathrm{G}}$-monomethyl-L-arginine. J Exp Med 1994;179:651-660. [PubMed: 7507509]

1355. Weinstein DM, Mihm MJ, Bauer JA. Cardiac peroxynitrite formation and left ventricular dysfunction following doxorubicin treatment in mice. J Pharmacol Exp Ther 2000;294:396-401. [PubMed: 10871338]

1356. Weiss JN, Korge P, Honda HM, Ping P. Role of the mitochondrial permeability transition in myocardial disease. Circ Res 2003;93:292-301. [PubMed: 12933700]

1357. Weksler ME, Gouras G, Relkin NR, Szabo P. The immune system, amyloid-beta peptide, Alzheimer's disease. Immunol Rev 2005;205:244-256. [PubMed: 15882358]

1358. Wendt S, Schlattner U, Wallimann T. Differential effects of peroxynitrite on human mitochondrial creatine kinase isoenzymes. Inactivation, octamer destabilization, identification of involved residues. J Biol Chem 2003;278:1125-1130. [PubMed: 12401781]

1359. Weydt P, Moller T. Neuroinflammation in the pathogenesis of amyotrophic lateral sclerosis. Neuroreport 2005;16:527-531. [PubMed: 15812300]

1360. Whalen MJ, Clark RS, Dixon CE, Robichaud P, Marion DW, Vagni V, Graham SH, Virag L, Hasko G, Stachlewitz R, Szabo C, Kochanek PM. Reduction of cognitive and motor deficits after traumatic brain injury in mice deficient in poly(ADP-ribose) polymerase. J Cereb Blood Flow Metab 1999;19:835-842. [PubMed: 10458590]

1361. Wheeler MD, Katuna M, Smutney OM, Froh M, Dikalova A, Mason RP, Samulski RJ, Thurman RG. Comparison of the effect of adenoviral delivery of three superoxide dismutase genes against hepatic ischemia-reperfusion injury. Hum Gene Ther 2001;12:2167-2177. [PubMed: 11779401]

1362. Wheeler-Jones CP. Cell signalling in the cardiovascular system: an overview. Heart 2005;91:13661374. [PubMed: 16162635]

1363. White BC, Sullivan JM, DeGracia DJ, O’Neil BJ, Neumar RW, Grossman LI, Rafols JA, Krause GS. Brain ischemia and reper-fusion: molecular mechanisms of neuronal injury. J Neurol Sci 2000;179:1-33. [PubMed: 11054482]

1364. White CR, Brock TA, Chang LY, Crapo J, Briscoe P, Ku D, Bradley WA, Gianturco SH, Gore J, Freeman BA, Tarpey MM. Superoxide and peroxynitrite in atherosclerosis. Proc Natl Acad Sci USA 1994;91:1044-1048. [PubMed: 8302829]

1365. White CR, Moellering D, Patel RP, Kirk M, Barnes S, Darley-Usmar VM. Formation of the NO donors glyceryl mononitrate and glyceryl mononitrite from the reaction of peroxynitrite with glycerol. Biochem J 1997;328:517-524. [PubMed: 9371710]

1366. Whiteman M, Armstrong JS, Cheung NS, Siau JL, Rose P, Schantz JT, Jones DP, Halliwell B. Peroxynitrite mediates calcium-dependent mitochondrial dysfunction and cell death via activation of calpains. FASEB J 2004;18:1395-1397. [PubMed: 15240564] 
1367. Whiteman M, Halliwell B. Protection against peroxynitrite-dependent tyrosine nitration and alpha 1-antiproteinase inactivation by ascorbic acid. A comparison with other biological antioxidants. Free Radic Res 1996;25:275-283. [PubMed: 8889493]

1368. Whiteman M, Kaur H, Halliwell B. Protection against peroxynitrite dependent tyrosine nitration and alpha 1-antiproteinase inactivation by some anti-inflammatory drugs and by the antibiotic tetracycline. Ann Rheum Dis 1996;55:383-387. [PubMed: 8694578]

1369. Whiteman M, Tritschler H, Halliwell B. Protection against peroxynitrite-dependent tyrosine nitration and alpha 1-antiproteinase inactivation by oxidized and reduced lipoic acid. FEBS Lett 1996;379:74-76. [PubMed: 8566234]

1370. Wild S, Roglic G, Green A, Sicree R, King H. Global prevalence of diabetes: estimates for the year 2000 and projections for 2030. Diabetes Care 2004;27:1047-1053. [PubMed: 15111519]

1371. Wildhirt SM, Weis M, Schulze C, Conrad N, Pehlivanli S, Rieder G, Enders G, von Scheidt W, Reichart B. Expression of endomyocardial nitric oxide synthase and coronary endothelial function in human cardiac allografts. Circulation 2001;104(Suppl I):I-336-I-343. [PubMed: 11568079]

1372. Willerson JT, Kereiakes DJ. Endothelial dysfunction. Circulation 2003;108:2060-2061. [PubMed: 14581385]

1373. Willmot M, Gibson C, Gray L, Murphy S, Bath P. Nitric oxide synthase inhibitors in experimental ischemic stroke and their effects on infarct size and cerebral blood flow: a systematic review. Free Radic Biol Med 2005;39:412-425. [PubMed: 15993340]

1374. Willmot M, Gray L, Gibson C, Murphy S, Bath PM. A systematic review of nitric oxide donors and L-arginine in experimental stroke; effects on infarct size and cerebral blood flow. Nitric Oxide 2005;12:141-149. [PubMed: 15797842]

1375. Wink D, Kasprzak K, Maragos C, Elespuru R, Misra M, Dunams T, Cebula T, Koch W, Andrews A, Allen J, Keefer L. DNA deaminating ability and genotoxicity of nitric oxide and its progenitors. Science 1991;254:1001-1003. [PubMed: 1948068]

1376. Wink DA, Darbyshire JF, Nims RW, Saavedra JE, Ford PC. Reactions of the bioregulatory agent nitric oxide in oxygenated aqueous media: determination of the kinetics for oxidation and nitrosation by intermediates generated in the $\mathrm{NO} / \mathrm{O}_{2}$ reaction. Chem Res Toxicol 1993;6:23-27. [PubMed: 8448345]

1377. Wise DL, Houghton G. Diffusion coefficients of neon, krypton, xenon, carbon monoxide and nitric oxide in water at $10-60^{\circ} \mathrm{C}$. Chem Eng Sci 1968;23:1211-1216.

1378. Wolin MS. Interactions of oxidants with vascular signaling systems. Arterioscler Thromb Vasc Biol 2000;20:1430-1442. [PubMed: 10845855]

1379. Wolin MS. Loss of vascular regulation by soluble guanylate cyclase is emerging as a key target of the hypertensive disease process. Hypertension 2005;45:1068-1069. [PubMed: 15883231]

1380. Wolin MS. Reactive oxygen species and vascular signal transduction mechanisms. Microcirculation 1996;3:1-17. [PubMed: 8846267]

1381. Wolin MS, Ahmad M, Gupte SA. Oxidant and redox signaling in vascular oxygen sensing mechanisms: basic concepts, current controversies, potential importance of cytosolic NADPH. Am J Physiol Lung Cell Mol Physiol 2005;289:L159-L173. [PubMed: 16002998]

1382. Wolin MS, Ahmad M, Gupte SA. The sources of oxidative stress in the vessel wall. Kidney Int 2005;67:1659-1661. [PubMed: 15840006]

1383. Wolin MS, Xie YW, Hintze TH. Nitric oxide as a regulator of tissue oxygen consumption. Curr Opin Nephrol Hypertens 1999;8:97-103. [PubMed: 9914866]

1384. Wong NK, Strong MJ. Nitric oxide synthase expression in cervical spinal cord in sporadic amyotrophic lateral sclerosis. Eur J Cell Biol 1998;77:338-343. [PubMed: 9930658]

1385. Wood J, Garthwaite J. Models of the diffusional spread of nitric oxide: implications for neural nitric oxide signalling and its pharmacological properties. Neuropharmacology 1994;33:12351244. [PubMed: 7870284]

1386. Woodman CR, Price EM, Laughlin MH. Aging induces muscle-specific impairment of endothelium-dependent dilation in skeletal muscle feed arteries. J Appl Physiol 2002;93:16851690. [PubMed: 12381754]

1387. Woolum JC, Tiezzi E, Commoner B. Electron spin resonance of iron-nitric oxide complexes with amino acids, peptides and proteins. Biochim Biophys Acta 1968;160:311-320. [PubMed: 4300590] 
1388. Wu G. Nitric oxide synthesis and the effect of aminoguanidine and $N^{\mathrm{G}}$-monomethyl-L-arginine on the onset of diabetes in the spontaneously diabetic BB rat. Diabetes 1995;44:360-364. [PubMed: 7533735]

1389. Wu M, Pritchard KA Jr, Kaminski PM, Fayngersh RP, Hintze TH, Wolin MS. Involvement of nitric oxide and nitrosothiols in relaxation of pulmonary arteries to peroxynitrite. Am J Physiol Heart Circ Physiol 1994;266:H2108-H2113.

1390. Wu W, Chen Y, Hazen SL. Eosinophil peroxidase nitrates protein tyrosyl residues. Implications for oxidative damage by nitrating intermediates in eosinophilic inflammatory disorders. J Biol Chem 1999;274:25933-25944. [PubMed: 10464338]

1391. Wykes V, Bellamy TC, Garthwaite J. Kinetics of nitric oxide-cyclic GMP signalling in CNS cells and its possible regulation by cyclic GMP. J Neurochem 2002;83:37-47. [PubMed: 12358727]

1392. Xia Y, Dawson VL, Dawson TM, Snyder SH, Zweier JL. Nitric oxide synthase generates superoxide and nitric oxide in arginine-depleted cells leading to peroxynitrite-mediated cellular injury. Proc Natl Acad Sci USA 1996;93:6770-6774. [PubMed: 8692893]

1393. Xia Y, Zweier JL. Superoxide and peroxynitrite generation from inducible nitric oxide synthase in macrophages. Proc Natl Acad Sci USA 1997;94:6954-6958. [PubMed: 9192673]

1394. Xie J, Roddy P, Rife TK, Murad F, Young AP. Two closely linked but separable promoters for human neuronal nitric oxide synthase gene transcription. Proc Natl Acad Sci USA 1995;92:12421246. [PubMed: 7532307]

1395. Xie QW, Cho HJ, Calaycay J, Mumford RA, Swiderek KM, Lee TD, Ding A, Troso T, Nathan C. Cloning and characterization of inducible nitric oxide synthase from mouse macrophages. Science 1992;256:225-228. [PubMed: 1373522]

1396. Xie YW, Kaminski PM, Wolin MS. Inhibition of rat cardiac muscle contraction and mitochondrial respiration by endogenous peroxynitrite formation during posthypoxic reoxygenation. Circ Res 1998;82:891-897. [PubMed: 9576108]

1397. Xu S, Ying J, Jiang B, Guo W, Adachi T, Sharov V, Lazar H, Menzoian J, Knyushko TV, Bigelow D, Schoneich C, Cohen RA. Detection of sequence-specific tyrosine nitration of manganese SOD and SERCA in cardiovascular disease and aging. Am J Physiol Heart Circ Physiol 2006;290:H2220-H2227. [PubMed: 16399855]

1398. Xu Y, Armstrong SJ, Arenas IA, Pehowich DJ, Davidge ST. Cardioprotection by chronic estrogen or superoxide dismutase mimetic treatment in the aged female rat. Am J Physiol Heart Circ Physiol 2004;287:H165-H171. [PubMed: 14988070]

1399. Xu Y, Huang S, Liu ZG, Han J. Poly(ADP-ribose) polymerase-1 signaling to mitochondria in necrotic cell death requires RIP1/TRAF2-mediated JNK1 activation. J Biol Chem 2006;281:87888795. [PubMed: 16446354]

1400. Yabe Y, Kobayashi N, Nishihashi T, Takahashi R, Nishikawa M, Takakura Y, Hashida M. Prevention of neutrophil-mediated hepatic ischemia/reperfusion injury by superoxide dismutase and catalase derivatives. J Pharmacol Exp Ther 2001;298:894-899. [PubMed: 11504782]

1401. Yamaguchi T, Yoshida N, Ichiishi E, Sugimoto N, Naito Y, Yoshikawa T. Differing effects of two nitric oxide synthase inhibitors on experimental colitis. Hepatogastroenterology 2001;48:118-122. [PubMed: 11268945]

1402. Yamaguchi Y, Yoshikawa N, Kagota S, Nakamura K, Haginaka J, Kunitomo M. Elevated circulating levels of markers of oxidative-nitrative stress and inflammation in a genetic rat model of metabolic syndrome. Nitric Oxide. In press

1403. Yamakura F, Ikeda K. Modification of tryptophan and tryptophan residues in proteins by reactive nitrogen species. Nitric Oxide 2006;14:152-161. [PubMed: 16140551]

1404. Yamakura F, Matsumoto T, Ikeda K, Taka H, Fujimura T, Murayama K, Watanabe E, Tamaki M, Imai T, Takamori K. ONitrated and oxidized products of a single tryptophan residue in human $\mathrm{Cu}, \mathrm{Zn}$-superoxide dismutase treated with either peroxynitrite-carbon dioxide or myeloperoxidasehydrogen peroxidenitrite. J Biochem 2005;138:57-69. [PubMed: 16046449]

1405. Yamakura F, Taka H, Fujimura T, Murayama K. Inactivation of human manganese-superoxide dismutase by peroxynitrite is caused by exclusive nitration of tyrosine 34 to 3-nitrotyrosine. J Biol Chem 1998;273:14085-14089. [PubMed: 9603906] 
1406. Yamashita H, Akamine S, Sumida Y, Inoue M, Sawada T, Nagayasu T, Oka T. Inhaled nitric oxide attenuates apoptosis in ischemia-reperfusion injury of the rabbit lung. Ann Thorac Surg 2004;78:292-297. [PubMed: 15223447]

1407. Yamashita T, Ando Y, Obayashi K, Uchino M, Ando M. Changes in nitrite and nitrate (NO2-/ NO3-) levels in cerebrospinal fluid of patients with multiple sclerosis. J Neurol Sci 1997;153:3234. [PubMed: 9455975]

1408. Yan C, Huang A, Wu Z, Kaminski PM, Wolin MS, Hintze TH, Kaley G, Sun D. Increased superoxide leads to decreased flow-induced dilation in resistance arteries of Mn-SOD-deficient mice. Am J Physiol Heart Circ Physiol 2005;288:H2225-H2231. [PubMed: 15637112]

1409. Yang B, Larson DF, Watson RR. Modulation of iNOS activity in age-related cardiac dysfunction. Life Sci 2004;75:655-667. [PubMed: 15172175]

1410. Yang G, Chan PH, Chen J, Carlson E, Chen SF, Weinstein P, Epstein CJ, Kamii H. Human copperzinc superoxide dismutase transgenic mice are highly resistant to reperfusion injury after focal cerebral ischemia. Stroke 1994;25:165-170. [PubMed: 8266365]

1411. Yang H, Shi M, Story J, Richardson A, Guo Z. Food restriction attenuates age-related increase in the sensitivity of endothelial cells to oxidized lipids. J Gerontol A Biol Sci Med Sci 2004;59:316323. [PubMed: 15071074]

1412. Yardim-Akaydin S, Kesimer M, Imren E, Sepici A, Simsek B, Torun M. Urate oxidation during percutaneous transluminal coronary angioplasty and thrombolysis in patients with coronary artery disease. Clin Chim Acta 2005;362:131-137. [PubMed: 15992789]

1413. Yasmin W, Strynadka KD, Schulz R. Generation of peroxynitrite contributes to ischemiareperfusion injury in isolated rat hearts. Cardiovasc Res 1997;33:422-432. [PubMed: 9074708]

1414. Ye YZ, Strong M, Huang ZQ, Beckman JS. Antibodies that recognize nitrotyrosine. Methods Enzymol 1996;269:201-209. [PubMed: 8791650]

1415. Yellon DM, Hausenloy DJ. Realizing the clinical potential of ischemic preconditioning and postconditioning. Nat Clin Pract Cardiovasc Med 2005;2:568-575. [PubMed: 16258568]

1416. Yokozawa T, Chen CP, Rhyu DY, Tanaka T, Park JC, Kitani K. Potential of sanguiin H-6 against oxidative damage in renal mitochondria and apoptosis mediated by peroxynitrite in vivo. Nephron 2002;92:133-141. [PubMed: 12187096]

1417. Yokozawa T, Ishida A, Kashiwada Y, Cho EJ, Kim HY, Ikeshiro Y. Coptidis Rhizoma: protective effects against peroxynitrite-induced oxidative damage and elucidation of its active components. J Pharm Pharmacol 2004;56:547-556. [PubMed: 15099450]

1418. Yokozawa T, Rhyu DY, Cho EJ. (-)-Epicatechin 3-O-gallate ameliorates the damages related to peroxynitrite production by mechanisms distinct from those of other free radical inhibitors. J Pharm Pharmacol 2004;56:231-239. [PubMed: 15005882]

1419. Yonekura Y, Koshiishi I, Yamada K, Mori A, Uchida S, Nakamura T, Utsumi H. Association between the expression of inducible nitric oxide synthase by chondrocytes and its nitric oxidegenerating activity in adjuvant arthritis in rats. Nitric Oxide 2003;8:164-169. [PubMed: 12826065]

1420. Yoo K, Choi JW, Choi MS, Ryu MK, Park GH, Jeon MJ, Ko KH. Mitogen-activated protein kinases (MAPKs) mediate SIN-1/glucose deprivation-induced death in rat primary astrocytes. Arch Pharm Res 2005;28:942-947. [PubMed: 16178421]

1421. Yoshida T, Limmroth V, Irikura K, Moskowitz MA. The NOS inhibitor, 7-nitroindazole, decreases focal infarct volume but not the response to topical acetylcholine in pial vessels. J Cereb Blood Flow Metab 1994;14:924-929. [PubMed: 7523430]

1422. Yu H, Venkatarangan L, Wishnok JS, Tannenbaum SR. Quantitation of four guanine oxidation products from reaction of DNA with varying doses of peroxynitrite. Chem Res Toxicol 2005;18:1849-1857. [PubMed: 16359175]

1423. Yu J, deMuinck ED, Zhuang Z, Drinane M, Kauser K, Rubanyi GM, Qian HS, Murata T, Escalante B, Sessa WC. Endothelial nitric oxide synthase is critical for ischemic remodeling, mural cell recruitment, blood flow reserve. Proc Natl Acad Sci USA 2005;102:10999-11004. [PubMed: 16043715]

1424. Yu SW, Wang H, Poitras MF, Coombs C, Bowers WJ, Federoff HJ, Poirier GG, Dawson TM, Dawson VL. Mediation of poly(ADP-ribose) polymerase-1-dependent cell death by apoptosisinducing factor. Science 2002;297:259-263. [PubMed: 12114629] 
1425. Yu ZF, Bruce-Keller AJ, Goodman Y, Mattson MP. Uric acid protects neurons against excitotoxic and metabolic insults in cell culture, against focal ischemic brain injury in vivo. J Neurosci Res 1998;53:613-625. [PubMed: 9726432]

1426. Yue G, Lai PS, Yin K, Sun FF, Nagele RG, Liu X, Linask KK, Wang C, Lin KT, Wong PY. Colon epithelial cell death in 2,4,6-trinitrobenzenesulfonic acid-induced colitis is associated with increased inducible nitric-oxide synthase expression and peroxynitrite production. J Pharmacol Exp Ther 2001;297:915-925. [PubMed: 11356911]

1427. Yuen EC, Gunther EC, Bothwell M. Nitric oxide activation of TrkB through peroxynitrite. Neuroreport 2000;11:3593-3597. [PubMed: 11095525]

1428. Zafiriou O, McFarland M. Determination of trace levels of nitric oxide in aqueous solution. Anal Chem 1980;52:1662-1667.

1429. Zahmatkesh M, Kadkhodaee M, Arab HA, Shams S. Effects of co-administration of an iNOS inhibitor with a broad-spectrum reactive species scavenger in rat renal ischemia/reperfusion injury. Nephron Exp Nephrol 2006;103:119-125.

1430. Zhang C, Walker LM, Mayeux PR. Role of nitric oxide in lipopolysaccharide-induced oxidant stress in the rat kidney. Biochem Pharmacol 2000;59:203-209. [PubMed: 10810455]

1431. Zhang F, Slungaard A, Vercellotti GM, Iadecola C. Superoxide-dependent cerebrovascular effects of homocysteine. Am J Physiol Regul Integr Comp Physiol 1998;274:R1704-R1711.

1432. Zhang F, White JG, Iadecola C. Nitric oxide donors increase blood flow and reduce brain damage in focal ischemia: evidence that nitric oxide is beneficial in the early stages of cerebral ischemia. $\mathrm{J}$ Cereb Blood Flow Metab 1994;14:217-226. [PubMed: 8113318]

1433. Zhang L, Dawson VL, Dawson TM. Role of nitric oxide in Parkinson's disease. Pharmacol Ther 2006;109:33-41. [PubMed: 16005074]

1434. Zhang P, Wang YZ, Kagan E, Bonner JC. Peroxynitrite targets the epidermal growth factor receptor, Raf-1, MEK independently to activate MAPK. J Biol Chem 2000;275:22479-22486. [PubMed: 10801894]

1435. Zhang R, Ma J, Xia M, Zhu H, Ling W. Mild hyperhomocysteinemia induced by feeding rats diets rich in methionine or deficient in folate promotes early atherosclerotic inflammatory processes. $\mathrm{J}$ Nutr 2004;134:825-830. [PubMed: 15051832]

1436. Zhang X, Chen J, Graham SH, Du L, Kochanek PM, Draviam R, Guo F, Nathaniel PD, Szabo C, Watkins SC, Clark RS. Intranuclear localization of apoptosis-inducing factor (AIF) and large scale DNA fragmentation after traumatic brain injury in rats and in neuronal cultures exposed to peroxynitrite. J Neurochem 2002;82:181-191. [PubMed: 12091479]

1437. Zhang Y, Bissing JW, Xu L, Ryan AJ, Martin SM, Miller FJ Jr, Kregel KC, Buettner GR, Kerber RE. Nitric oxide synthase inhibitors decrease coronary sinus-free radical concentration and ameliorate myocardial stunning in an ischemia-reperfusion model. J Am Coll Cardiol 2001;38:546554. [PubMed: 11499751]

1438. Zhang Y, Davies LR, Martin SM, Coddington WJ, Miller FJ Jr, Buettner GR, Kerber RE. The nitric oxide donor $S$-nitroso- $N$-acetylpenicillamine (SNAP) increases free radical generation and degrades left ventricular function after myocardial ischemia-reperfusion. Resuscitation 2003;59:345-352. [PubMed: 14659604]

1439. Zhang Y, Wang H, Li J, Dong L, Xu P, Chen W, Neve RL, Volpe JJ, Rosenberg PA. Intracellular zinc release and ERK phosphorylation are required upstream of 12-lipoxygenase activation in peroxynitrite toxicity to mature rat oligodendrocytes. J Biol Chem 2006;281:9460-9470. [PubMed: 16431921]

1440. Zhang Y, Wang H, Li J, Jimenez DA, Levitan ES, Aizenman E, Rosenberg PA. Peroxynitriteinduced neuronal apoptosis is mediated by intracellular zinc release and 12-lipoxygenase activation. J Neurosci 2004;24:10616-10627. [PubMed: 15564577]

1441. Zhang YH, Lin JX, Vilcek J. Interleukin-6 induction by tumor necrosis factor and interleukin-1 in human fibroblasts involves activation of a nuclear factor binding to a kappa B-like sequence. Mol Cell Biol 1990;10:3818-3823. [PubMed: 2192263]

1442. Zhang YJ, Xu YF, Liu YH, Yin J, Li HL, Wang Q, Wang JZ. Peroxynitrite induces Alzheimerlike tau modifications and accumulation in rat brain and its underlying mechanisms. FASEB J 2006;20:1431-1442. [PubMed: 16816118] 
1443. Zhang ZG, Chopp M, Gautam S, Zaloga C, Zhang RL, Schmidt HH, Pollock JS, Forstermann U. Upregulation of neuronal nitric oxide synthase and mRNA, selective sparing of nitric oxide synthase-containing neurons after focal cerebral ischemia in rat. Brain Res 1994;654:85-95. [PubMed: 7526966]

1444. Zhang ZG, Chopp M, Zaloga C, Pollock JS, Forstermann U. Cerebral endothelial nitric oxide synthase expression after focal cerebral ischemia in rats. Stroke 1993;24:2016-2021. [PubMed: 7504335]

1445. Zhang ZG, Reif D, Macdonald J, Tang WX, Kamp DK, Gentile RJ, Shakespeare WC, Murray RJ, Chopp M. ARL 17477, a potent and selective neuronal NOS inhibitor decreases infarct volume after transient middle cerebral artery occlusion in rats. J Cereb Blood Flow Metab 1996;16:599_ 604. [PubMed: 8964798]

1446. Zhao H, Joseph J, Fales HM, Sokoloski EA, Levine RL, Vasquez-Vivar J, Kalyanaraman B. Detection and characterization of the product of hydroethidine and intracellular superoxide by HPLC and limitations of fluorescence. Proc Natl Acad Sci USA 2005;102:5727-5732. [PubMed: 15824309]

1447. Zhao S, Zhang Y, Gu Y, Lewis DF, Wang Y. Heme oxygenase-1 mediates up-regulation of adhesion molecule expression induced by peroxynitrite in endothelial cells. J Soc Gynecol Invest 2004;11:465-471.

1448. Zhao Y, Patzer A, Herdegen T, Gohlke P, Culman J. Activation of cerebral peroxisome proliferatoractivated receptors gamma promotes neuroprotection by attenuation of neuronal cyclooxygenase-2 overexpression after focal cerebral ischemia in rats. FASEB J 2006;20:1162-1175. [PubMed: 16770015]

1449. Zhao ZQ, Corvera JS, Halkos ME, Kerendi F, Wang NP, Guyton RA, Vinten-Johansen J. Inhibition of myocardial injury by ischemic postconditioning during reperfusion: comparison with ischemic preconditioning. Am J Physiol Heart Circ Physiol 2003;285:H579-H588. [PubMed: 12860564]

1450. Zhou JL, Zhu XG, Ling YL, Li Q. Melatonin reduces peroxynitrite-induced injury in aortic smooth muscle cells. Acta Pharmacol 2004;25:186-190.

1451. Zhu C, Wang X, Qiu L, Peeters-Scholte C, Hagberg H, Blomgren K. Nitrosylation precedes caspase-3 activation and translocation of apoptosis-inducing factor in neonatal rat cerebral hypoxiaischaemia. J Neurochem 2004;90:462-471. [PubMed: 15228602]

1452. Zhu C, Xu F, Wang X, Shibata M, Uchiyama Y, Blomgren K, Hagberg H. Different apoptotic mechanisms are activated in male and female brains after neonatal hypoxia-ischaemia. J Neurochem 2006;96:1016-1027. [PubMed: 16412092]

1453. Zhu S, Basiouny KF, Crow JP, Matalon S. Carbon dioxide enhances nitration of surfactant protein A by activated alveolar macrophages. Am J Physiol Lung Cell Mol Physiol 2000;278:L1025L1031. [PubMed: 10781434]

1454. Zhu T, Ling Y, Zhao R. Role of peroxynitrite on airway hyperresponsiveness in asthmatic guineapigs. Zhonghua Jie He He Hu Xi Za Zhi 2000;23:538-541. [PubMed: 11778526]

1455. Zielonka J, Zhao H, Xu Y, Kalyanaraman B. Mechanistic similarities between oxidation of hydroethidine by Fremy's salt and superoxide: stopped-flow optical and EPR studies. Free Radic Biol Med 2005;39:853-863. [PubMed: 16140206]

1456. Zingarelli B, Cuzzocrea S, Szabo C, Salzman AL. Mercapto-ethylguanidine, a combined inhibitor of nitric oxide synthase and peroxynitrite scavenger, reduces trinitrobenzene sulfonic acid-induced colonic damage in rats. J Pharmacol Exp Ther 1998;287:1048-1055. [PubMed: 9864291]

1457. Zingarelli B, Day BJ, Crapo JD, Salzman AL, Szabo C. The potential role of peroxynitrite in the vascular contractile and cellular energetic failure in endotoxic shock. Br J Pharmacol 1997;120:259_ 267. [PubMed: 9117118]

1458. Zingarelli B, Hake PW, Burroughs TJ, Piraino G, O'Connor M, Denenberg A. Activator protein-1 signalling pathway and apoptosis are modulated by poly(ADP-ribose) polymerase- 1 in experimental colitis. Immunology 2004;113:509-517. [PubMed: 15554929]

1459. Zingarelli B, Hake PW, O’Connor M, Denenberg A, Wong HR, Kong S, Aronow BJ. Differential regulation of activator protein-1 and heat shock factor-1 in myocardial ischemia and reperfusion injury: role of poly(ADP-ribose) polymerase-1. Am J Physiol Heart Circ Physiol 2004;286:H1408H1415. [PubMed: 14670820] 
1460. Zingarelli B, Ischiropoulos H, Salzman AL, Szabo C. Amelioration by mercaptoethylguanidine of the vascular and energetic failure in haemorrhagic shock in the anesthetised rat. Eur J Pharmacol 1997;338:55-65. [PubMed: 9408003]

1461. Zingarelli B, O'Connor M, Hake PW. Inhibitors of poly(ADP-ribose) polymerase modulate signal transduction pathways in colitis. Eur J Pharmacol 2003;469:183-194. [PubMed: 12782201]

1462. Zingarelli B, O’Connor M, Wong H, Salzman AL, Szabo C. Peroxynitrite-mediated DNA strand breakage activates poly-adenosine diphosphate ribosyl synthetase and causes cellular energy depletion in macrophages stimulated with bacterial lipopolysaccharide. J Immunol 1996;156:350358. [PubMed: 8598485]

1463. Zingarelli B, Salzman AL, Szabo C. Protective effects of nicotinamide against nitric oxidemediated delayed vascular failure in endotoxic shock: potential involvement of polyADP-ribosyl synthetase. Shock 1996;5:258-264. [PubMed: 8721385]

1464. Zingarelli B, Szabo C, Salzman AL. Reduced oxidative and nitrosative damage in murine experimental colitis in the absence of inducible nitric oxide synthase. Gut 1999;45:199-209. [PubMed: 10403731]

1465. Ziolo MT, Katoh H, Bers DM. Expression of inducible nitric oxide synthase depresses betaadrenergic-stimulated calcium release from the sarcoplasmic reticulum in intact ventricular myocytes. Circulation 2001;104:2961-2966. [PubMed: 11739313]

1466. Zou M, Jendral M, Ullrich V. Prostaglandin endoperoxide-dependent vasospasm in bovine coronary arteries after nitration of prostacyclin synthase. Br J Pharmacol 1999;126:1283-1292. [PubMed: 10217520]

1467. Zou M, Yesilkaya A, Ullrich V. Peroxynitrite inactivates prostacyclin synthase by hemethiolatecatalyzed tyrosine nitration. Drug Metab Rev 1999;31:343-349. [PubMed: 10335439]

1468. Zou MH, Bachschmid M. Hypoxia-reoxygenation triggers coronary vasospasm in isolated bovine coronary arteries via tyrosine nitration of prostacyclin synthase. J Exp Med 1999;190:135-139. [PubMed: 10429677]

1469. Zou MH, Cohen R, Ullrich V. Peroxynitrite and vascular endothelial dysfunction in diabetes mellitus. Endothelium 2004;11:89-97. [PubMed: 15370068]

1470. Zou MH, Hou XY, Shi CM, Kirkpatick S, Liu F, Goldman MH, Cohen RA. Activation of 5'-AMPactivated kinase is mediated through c-Src and phosphoinositide 3-kinase activity during hypoxiareoxygenation of bovine aortic endothelial cells. Role of peroxynitrite. J Biol Chem 2003;278:34003-34010. [PubMed: 12824177]

1471. Zou MH, Hou XY, Shi CM, Nagata D, Walsh K, Cohen RA. Modulation by peroxynitrite of Aktand AMP-activated kinase-dependent Ser1179 phosphorylation of endothelial nitric oxide synthase. J Biol Chem 2002;277:32552-32557. [PubMed: 12107173]

1472. Zou MH, Leist M, Ullrich V. Selective nitration of prostacyclin synthase and defective vasorelaxation in atherosclerotic bovine coronary arteries. Am J Pathol 1999;154:1359-1365. [PubMed: 10329589]

1473. Zou MH, Shi C, Cohen RA. High glucose via peroxynitrite causes tyrosine nitration and inactivation of prostacyclin synthase that is associated with thromboxane/prostaglandin $\mathrm{H}(2)$ receptor-mediated apoptosis and adhesion molecule expression in cultured human aortic endothelial cells. Diabetes 2002;51:198-203. [PubMed: 11756341]

1474. Zou MH, Shi C, Cohen RA. Oxidation of the zinc-thiolate complex and uncoupling of endothelial nitric oxide synthase by peroxynitrite. J Clin Invest 2002;109:817-826. [PubMed: 11901190]

1475. Zou MH, Ullrich V. Peroxynitrite formed by simultaneous generation of nitric oxide and superoxide selectively inhibits bovine aortic prostacyclin synthase. FEBS Lett 1996;382:101-104. [PubMed: 8612727]

1476. Zouki C, Jozsef L, Ouellet S, Paquette Y, Filep JG. Peroxynitrite mediates cytokine-induced IL-8 gene expression and production by human leukocytes. J Leukoc Biol 2001;69:815-824. [PubMed: 11358991]

1477. Zouki C, Zhang SL, Chan JS, Filep JG. Peroxynitrite induces integrin-dependent adhesion of human neutrophils to endothelial cells via activation of the Raf-1/MEK/Erk pathway. FASEB J 2001;15:25-27. [PubMed: 11099490] 
1478. Zweier JL, Fertmann J, Wei G. Nitric oxide and peroxynitrite in postischemic myocardium. Antioxid Redox Signal 2001;3:11-22. [PubMed: 11294189] 


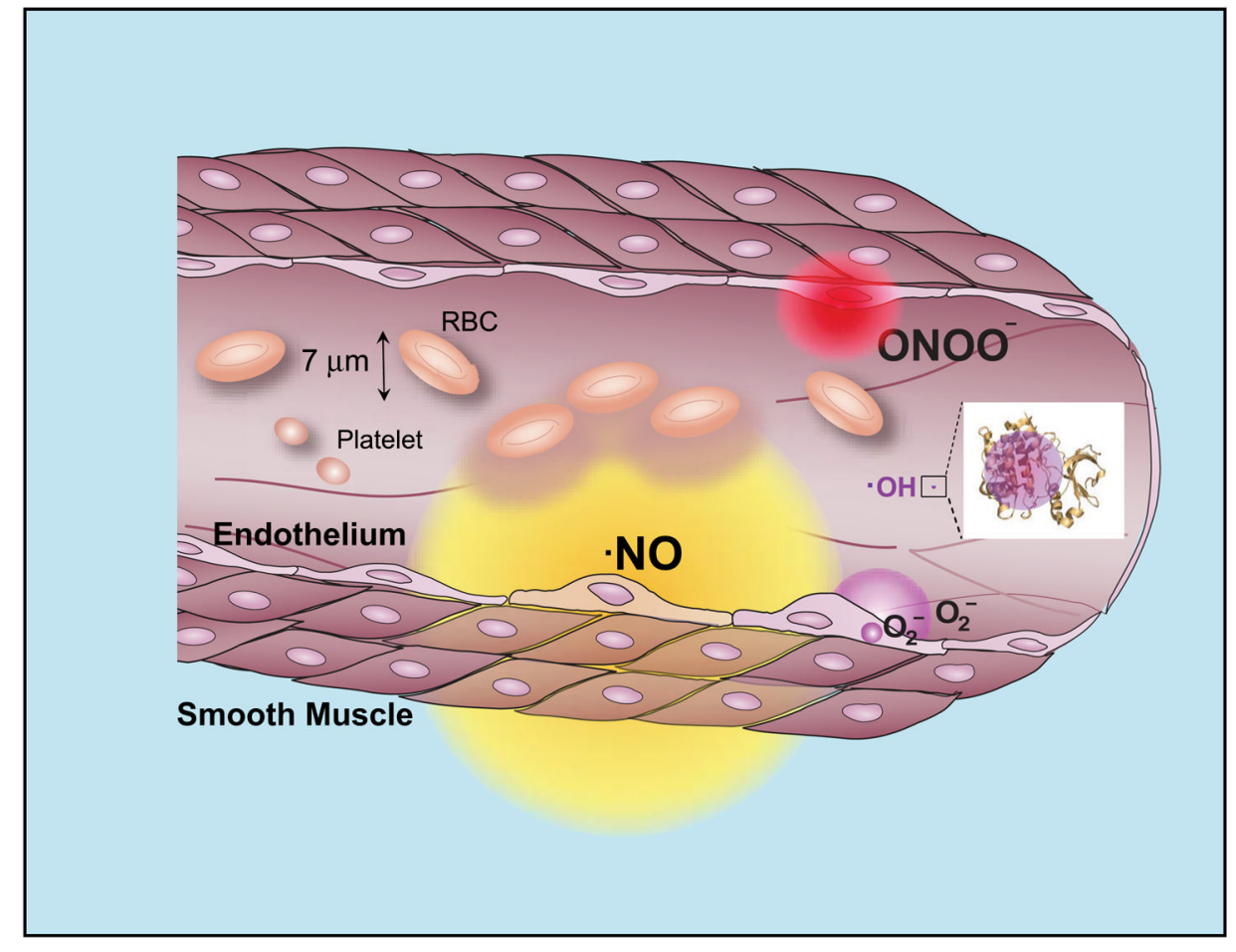

FIG. 1.

Cellular diffusion of superoxide, peroxynitrite, and hydroxyl radical within their estimated first half-lives. These circles indicate the extent to where the concentration of each species from a point source would decrease by $50 \%$. The diffusion of peroxynitrite accounts for its rapid reaction with carbon dioxide and with intracellular thiols. The diffusion distance for nitric oxide is calculated based on its half-life of $1 \mathrm{~s}$ in vivo, which results mostly from its diffusion into red blood cells. The diffusion distance for hydroxyl radical is about the same diameter as a small protein, or 10,000 times smaller than peroxynitrite. All of these estimates involve many approximations, but varying the estimated half-lives by 10 -fold would only alter the diameters by the square root of 10 or by 3.2 -fold. 


$$
\begin{aligned}
2 \mathrm{NO}+\mathrm{O}_{2} & \longrightarrow 2 \mathrm{NO}_{2} \\
\mathrm{NO}+\mathrm{O}_{2}^{-} & \longrightarrow \mathrm{ONOO}^{-}
\end{aligned}
$$

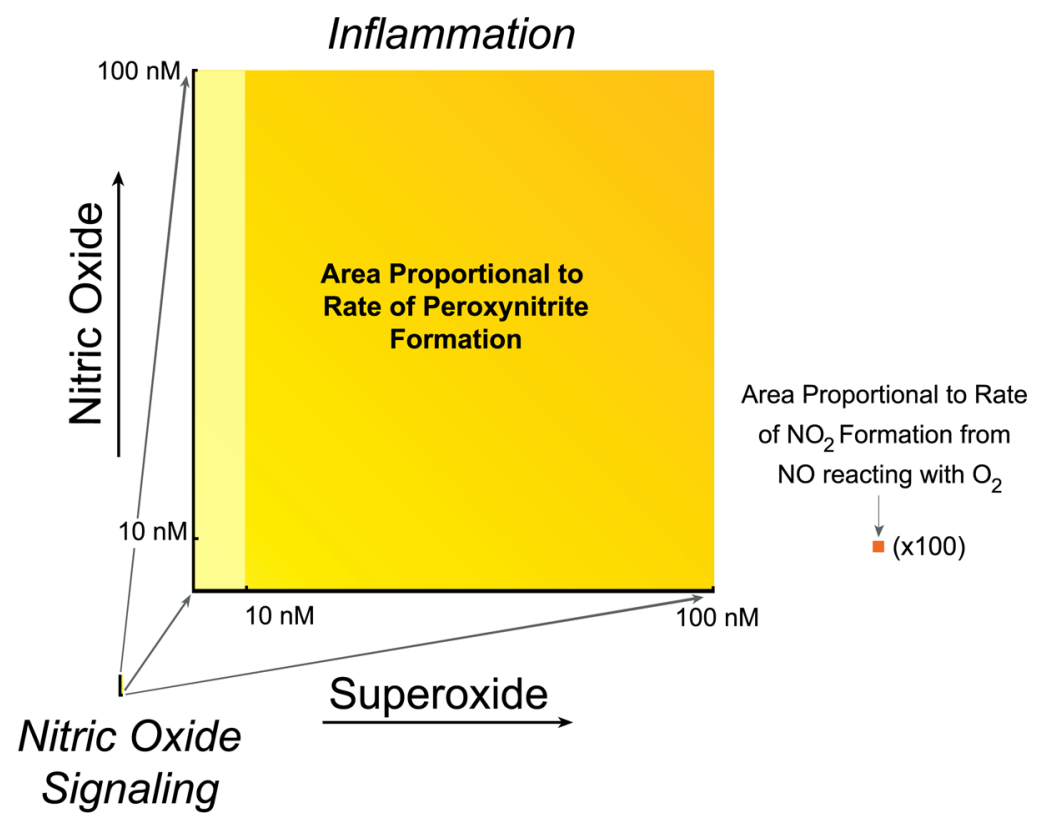

FIG. 2.

Comparison of oxidant production by the reaction of nitric oxide with superoxide versus oxygen. Both reactions are generally given equal weight, but this obscures the vast difference in oxidant productions because of the vast difference in rates. Because the formation of peroxynitrite depends on the product of the concentration of nitric oxide and superoxide, the rate of formation is proportional to the area. Left: estimate of peroxynitrite formation in the cytosol if a cell produces $10 \mathrm{nM}$ nitric oxide, sufficient to activate guanylate cyclase enough to cause at least $10 \%$ relaxation of vessels, using $0.1 \mathrm{nM}$ superoxide as an estimate of the basal steady-state concentration of superoxide (777). Right: increase in peroxynitrite formation if the formation of superoxide production increased either 100-fold (yellow) or 1,000-fold (yellow-orange), increases that can reasonably occur with the activation of NADPH oxidase. Nitric oxide is shown to increase only 10 -fold and could rise to $\sim 1 \mu \mathrm{M}$ in highly inflamed states. Far right (orange square): proportional area of nitrogen dioxide formation from $100 \mathrm{nM}$ nitric oxide reacting with oxygen (estimated to be $50 \mu \mathrm{M}$ in cells), which is magnified 100-fold. This rate is the faster rate occurring in hydrophobic membranes and would be 300 -fold smaller in solution (784). Pathways that stimulate the synthesis of superoxide vastly increase oxidant production compared with the reaction of nitric oxide with oxygen. 


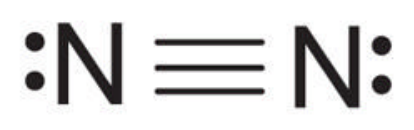

$\cdot \mathrm{N}=\mathrm{O}$

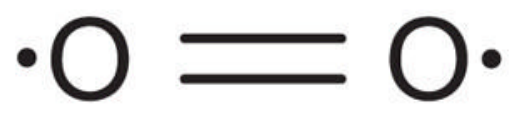

\section{3 bonds}

2.5 bonds

2 bonds

FIG. 3.

The chemical structure of nitric oxide is intermediate between molecular oxygen and nitrogen. The dot illustrates the unpaired electron on nitric oxide and two unpaired electrons on oxygen. These unpaired electrons are in antibonding orbitals, counteracting the three bonding orbitals characteristic of nitrogen gas. Thus nitric oxide has effectively 2.5 bonds and a slightly longer distance separating the nuclei. Oxygen has only two bonds and an even longer intranuclear distance. 


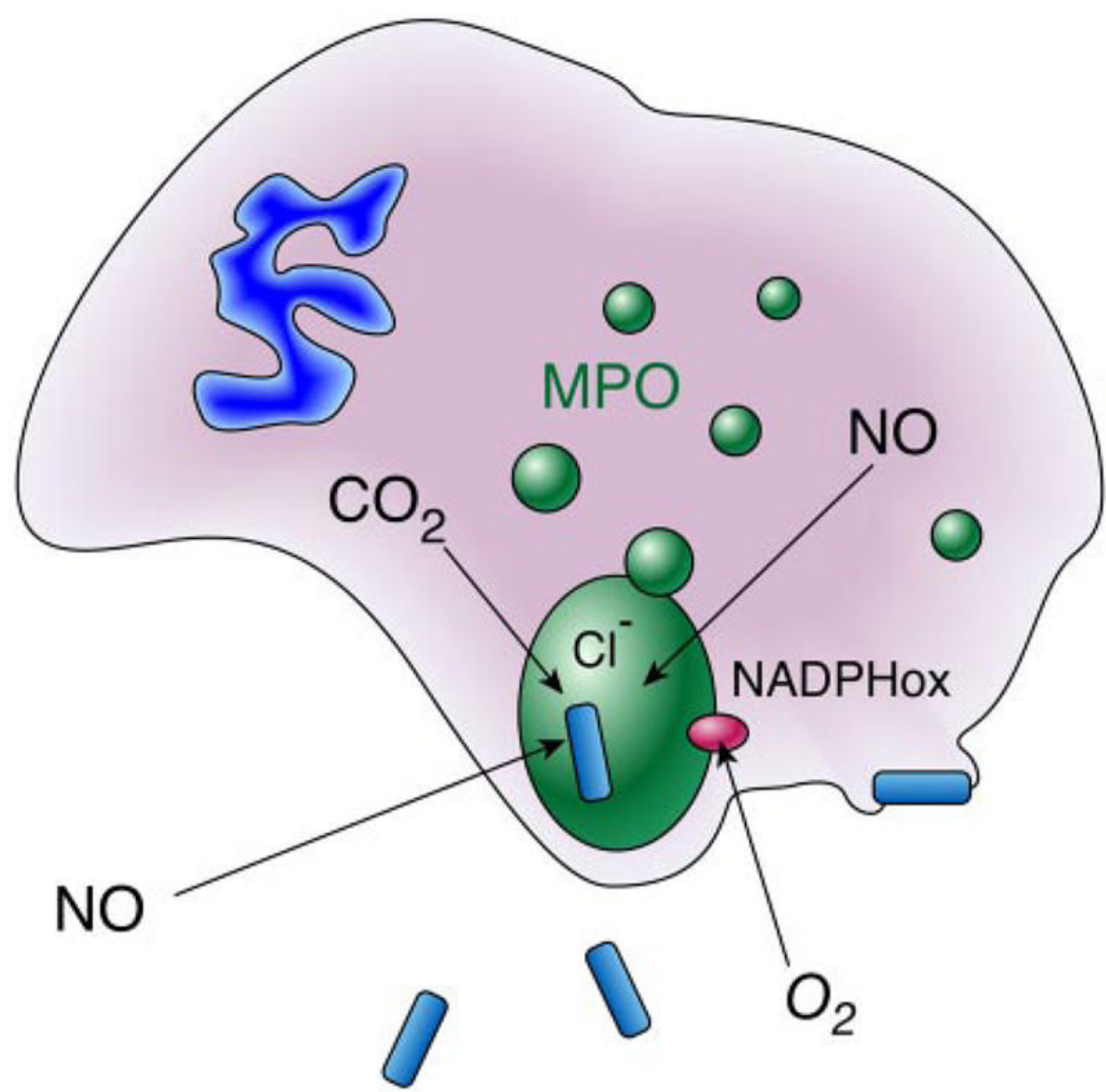

FIG. 4.

Diffusion of nitric oxide into the phagolysosome and the recycling of peroxynitrite-derived nitrite. Only a miniscule volume of extracellular fluid is engulfed into phagocytic vacuoles, which provides a limited amount of chloride as a substrate for myeloperoxidase. In contrast, nitric oxide can readily diffuse into the phagolysosome. Neutrophils produce superoxide by NADPH oxidases, but superoxide is unlikely to penetrate cell membranes or cell walls of pathogens, and can reversibly inactivate myeloperoxidase. Peroxynitrite is a substrate for myeloperoxidase and can reverse this inhibition. In addition, nitrite formed from peroxynitrite decomposition is entrapped within the phagolysosome and serves as an additional substrate for myeloperoxidase. Myeloperoxidase is not a predominant protein in macrophages, where formation of peroxynitrite from superoxide and nitric oxide appears to be a major mechanism of cytotoxicity. 


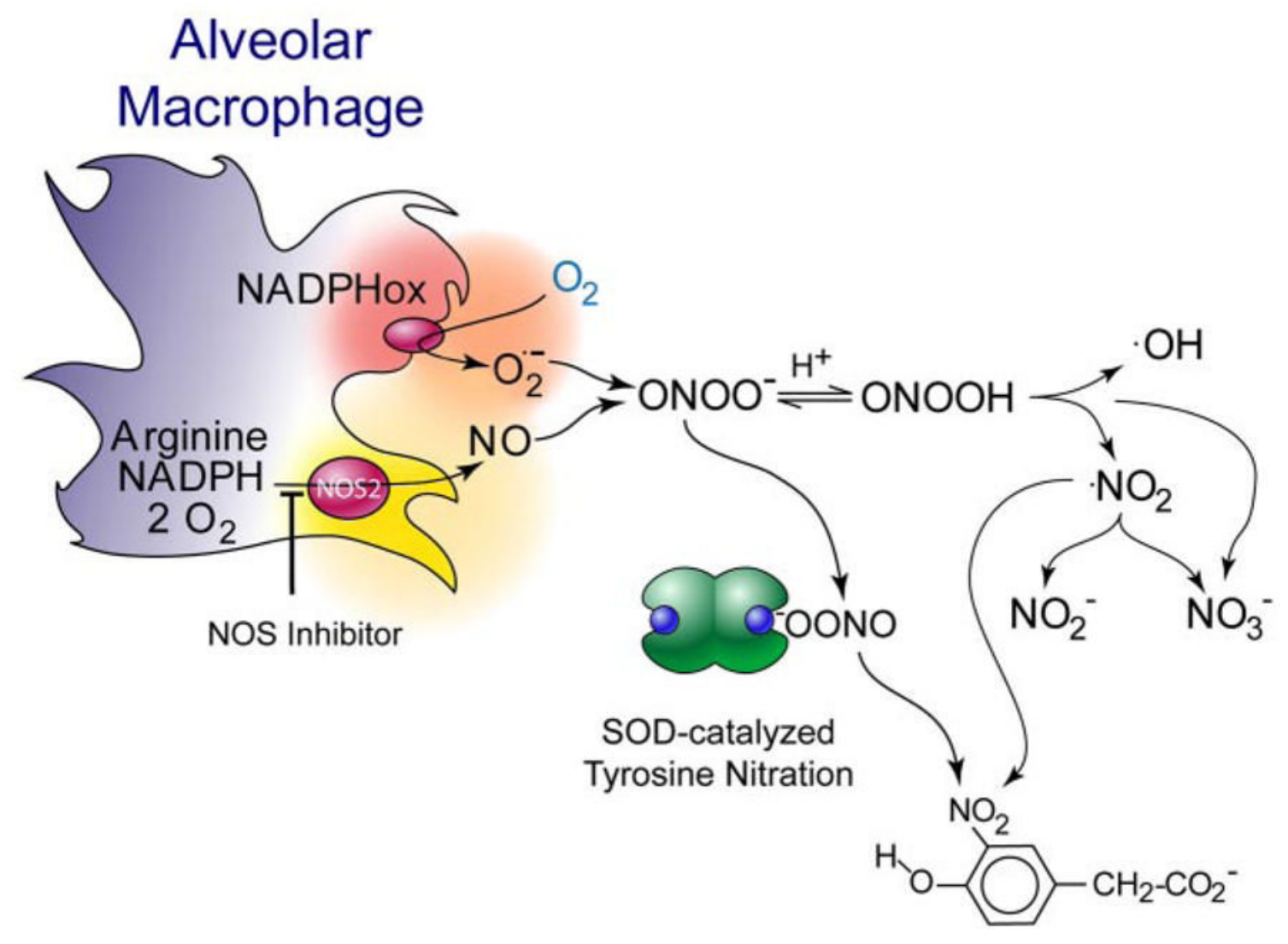

FIG. 5.

Alveolar macrophages produce peroxynitrite. When alveolar macrophages are stimulated to produce both superoxide and nitric oxide, peroxynitrite is quantitatively produced (611) as evidenced by the amount of nitric oxide and superoxide produced and the amount of oxygen consumed. Extracellular addition of superoxide dismutase (SOD) in high concentrations does not significantly reduce the amount of peroxynitrite formed and instead serves as a catalyst of tyrosine nitration. This suggests that superoxide produced at the membrane surface and nitric oxide diffusing through the membrane react at the membrane interface so quickly that SOD in the bulk phase cannot compete. 


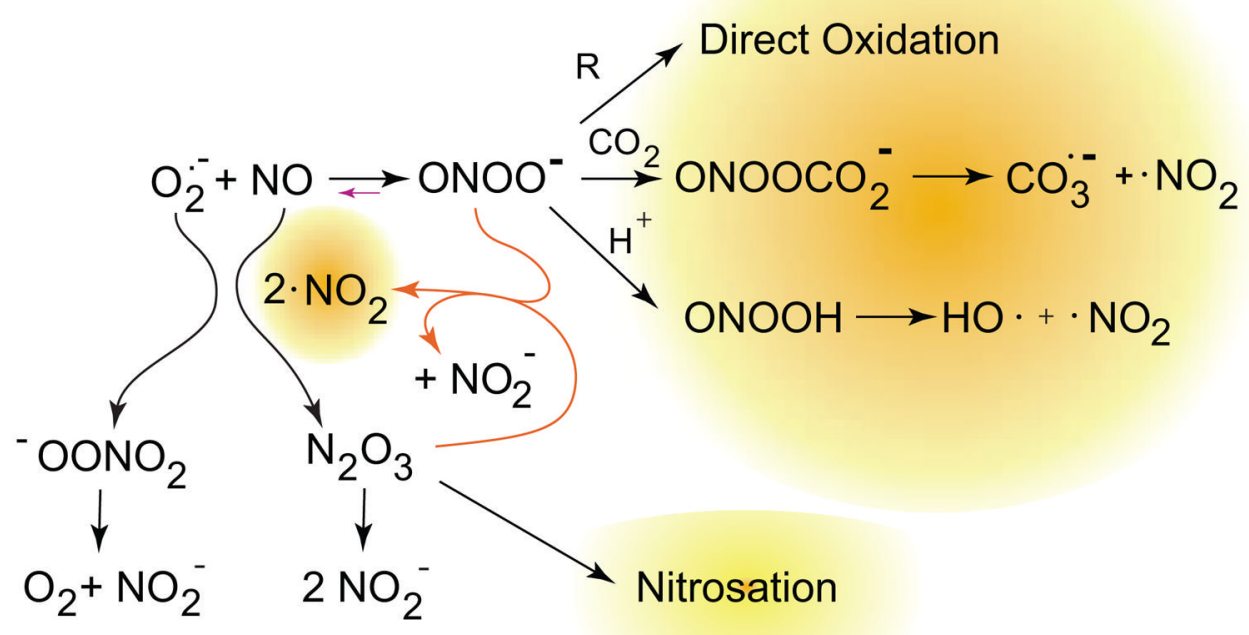

FIG. 6.

The interplay of nitric oxide, superoxide, peroxynitrite, and nitrogen dioxide. When nitric oxide and superoxide are both present, they may also react with nitrogen dioxide to form $\mathrm{N}_{2} \mathrm{O}_{3}$ and peroxynitrate. Peroxynitrate decomposes to give nitrite and oxygen, while $\mathrm{N}_{2} \mathrm{O}_{3}$ can react with thiols to give nitrosothiols or with hydroxide anion to give nitrite. Goldstein et al. (452) showed that it also reacts at a diffusion-limited rate with peroxynitrite to yield two molecules of nitrogen dioxide and one of nitrite. This creates a cycle to generate more nitrogen dioxide when bolus additions of peroxynitrite are added at neutral $\mathrm{pH}$ and substantially increases the number of potential reactions occurring. These same reactions will also occur in vivo, particularly when nitric oxide is produced faster than superoxide. 


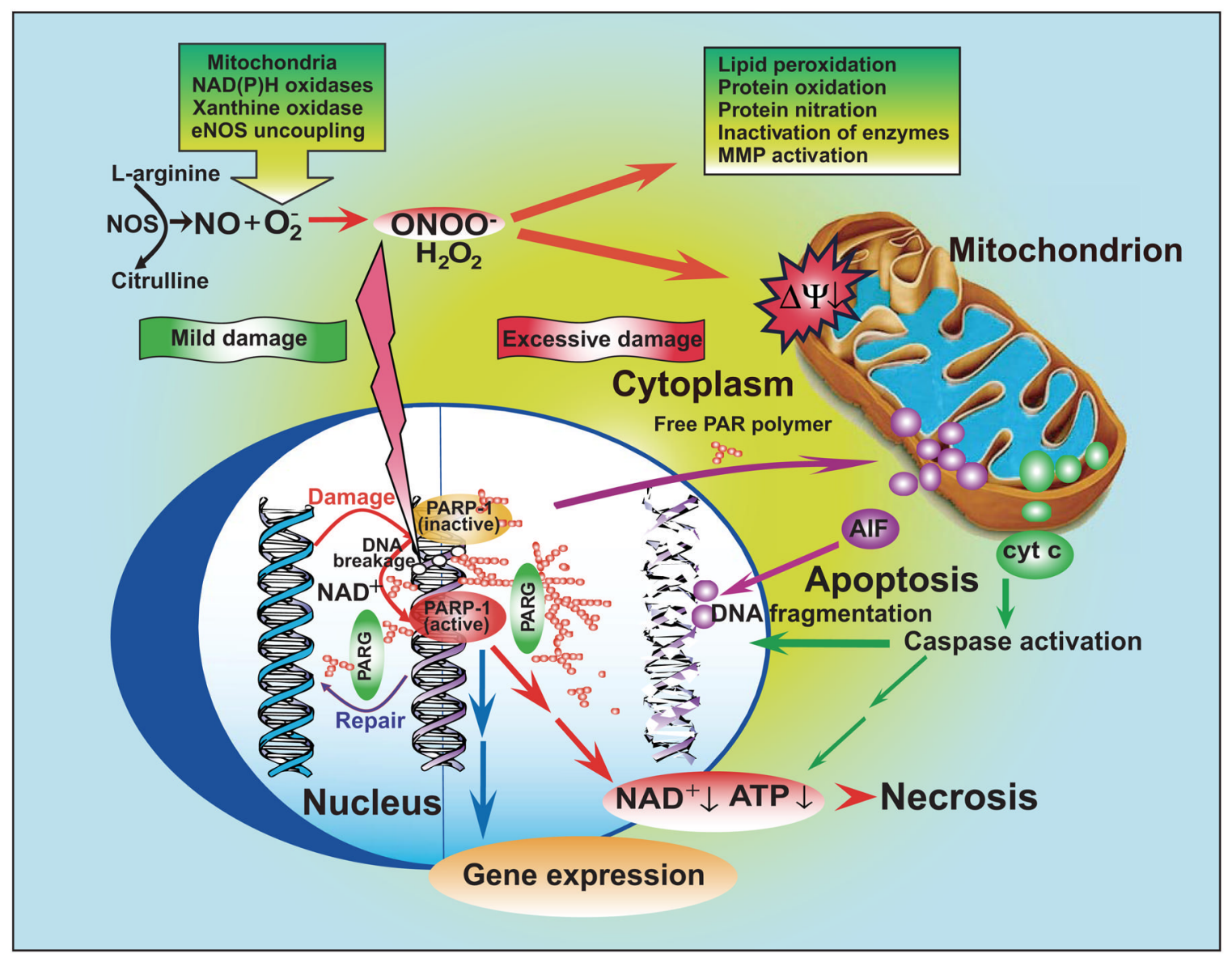

FIG. 7.

Molecular mechanisms of peroxynitrite-mediated cell death. A number of pathological conditions are associated with the simultaneous generation of nitric oxide (NO) and $\mathrm{O}_{2}{ }^{--}$. NO sources are restricted to the activity of the various NO synthases, whereas $\mathrm{O}_{2}{ }^{-}$arises from multiple sources, including electron leak from the mitochondria, NADPH oxidase, xanthine oxidase, and uncoupling of $\mathrm{NO}$ synthases. Once a flux of $\mathrm{NO}$ and $\mathrm{O}_{2}{ }^{--}$is produced simultaeously in close proximity, the generation of peroxynitrite is considerably enhanced. Peroxynitrite-dependent cytotoxicity is then mediated by a myriad of effects including lipid peroxidation, protein nitration and oxidation, DNA oxidative damage, activation of matrix metalloproteinases (MMP), and inactivation of a series of enzymes. Mitochondrial enzymes are particularly vulnerable to attacks by peroxynitrite, leading to reduced ATP formation and induction of mitochondrial permeability transition by opening of the permeability transition pore, which dissipates the mitochondrial membrane potential $\left(\Delta \psi_{\mathrm{m}}\right)$. These events result in cessation of electron transport and ATP formation, mitochondrial swelling, and permeabilization of the outer mitochondrial membrane, allowing the efflux of several proapoptotic molecules, including cytochrome $c$ and apoptosis-inducing factor (AIF). In turn, cytochrome $c$ and AIF activate a series of downstream effectors that eventually lead to the fragmentation of nuclear DNA. In addition to its damaging effects on mitochondria, peroxynitrite inflicts more or less severe oxidative injury to DNA, resulting in DNA strand breakage which in turn activates the nuclear enzyme poly(ADP-ribose) polymerase (PARP). Activated PARP consumes NAD to build-up poly(ADP-ribose) polymers (PAR), which are themselves rapidly metabolized by the activity of poly(ADP-ribose) glycohydrolase (PARG). Some free PAR may exit the nucleus and travel to the mitochondria, where they amplify the 
mitochondrial efflux of AIF (nuclear to mitochondria cross-talk). Mild damage of DNA activates the DNA repair machinery. On the contrary, once excessive oxidative and nitrosative stress-induced DNA damage occurs, like in various forms of reperfusion injury and other pathophysiological conditions, the cell may be executed by apoptosis in case of moderate PTP opening and PARP activation with preservation of cellular ATP, or by necrosis in the case of widespread PTP opening and PARP overactivation, leading to massive NAD consumption and collapse of cellular ATP. [Derived from Pacher et al. (995) with permission from Elsevier.] 


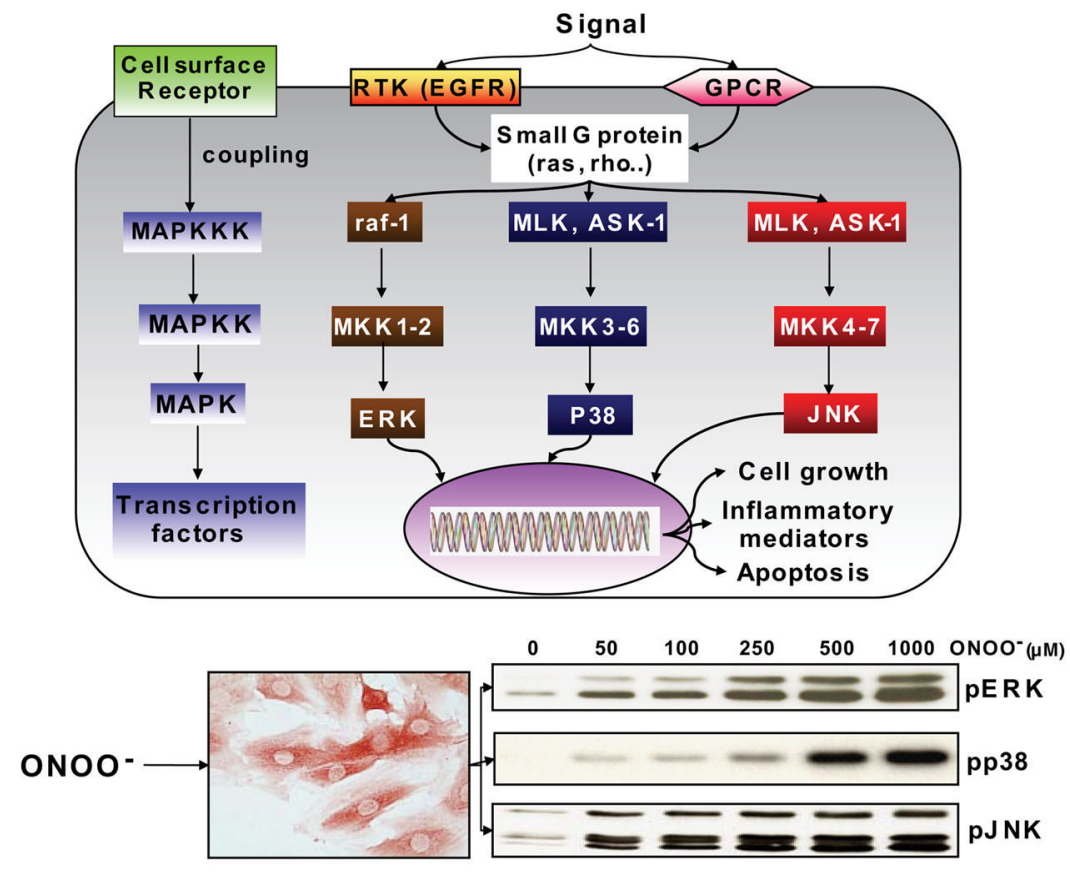

FIG. 8.

Schematic diagram of mitogen-activated protein kinase (MAPK) signaling and stimulating effects of peroxynitrite. MAPKs are activated by a dual phosphorylation at a specific tripeptide motif, as indicated on the left, mediated by a conserved protein kinase cascade, involving MAPK kinases (MAPKK or MKK) and MAPK kinase kinases (MAPKKK or MKKK). The activation of the upstream MKKK is mediated by various cell surface receptors, including $G$ protein-coupled receptors (GPCRs) and receptor tyrosine kinases (RTK), such as the receptor for epidermal growth factor (EGFR), which activate several small G proteins, such as Ras, Rho, Rac, and Cdc42. Three groups of MAPKs exist in mammalian cells, including extracellular signal-regulated protein kinase (ERK), p38 MAPK, and the c-Jun $\mathrm{NH}_{2}$-terminal kinase (JNK), whose upstream signaling intermediates include raf-1 and MKK1-2 (ERK pathway), MLK/Ask-1 (mixed lineage kinase/apoptosis-signal regulating kinase-1) and MEK 3-6 (p38), and MLK-1/Ask-1 and MKK 4-7 (JNK). Downstream targets of MAPKs are transcription factors, enzymes, and various proteins, which regulate cell growth, apoptosis, as well as inflammation. The Western blots at the bottom show the activation pattern of the three MAPKs, evidenced by their phosphorylation, induced in the cardiomyoblast cell line H9C2 by treatment with increasing concentrations of peroxynitrite. [Adapted from Pesse et al. (1024).] 


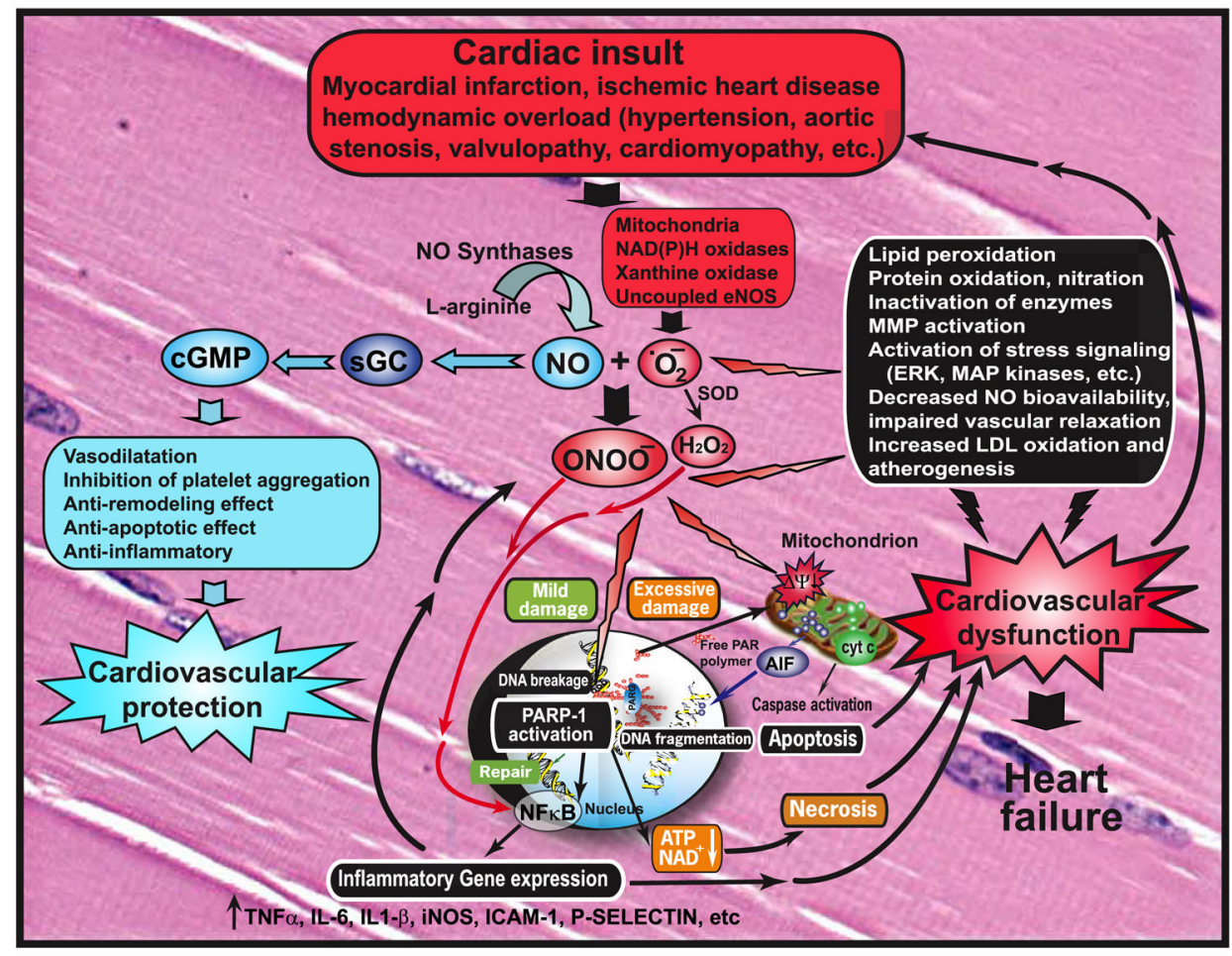

FIG. 9.

Role of nitric oxide (NO) and peroxynitrite in cardiovascular pathophysiology. On the one hand, NO by activating soluble guanylate cyclase (sGC)-cGMP signal transduction pathway mediates various physiological/beneficial effects in the cardiovascular system including vasodilation, inhibition of platelet aggregation, anti-inflammatory, antiremodelling, and antiapoptotic effects. On the other hand, under pathological conditions associated with increased oxidative stress and inflammation (myocardial infarction, ischemic heart disease, myocarditis, cardiomyopathy, hypertension, etc.), $\mathrm{NO}$ and superoxide $\left(\mathrm{O}_{2}{ }^{{ }^{-}-}\right)$react to form peroxynitrite $\left(\mathrm{ONOO}^{-}\right)$which induces cell damage via lipid peroxidation, inactivation of enzymes and other proteins by oxidation and nitration, and also activation of stress signaling, matrix metalloproteinases (MMPs) among others (see also Table 2). Peroxynitrite also triggers the release of proapoptotic factors such as cytochrome $c$ and apoptosis-inducing factor (AIF) from the mitochondria, which mediate caspase-dependent and -independent apoptotic death pathways. Moreover, peroxynitrite, in concert with other oxidants, causes stand breaks in DNA, activating the nuclear enzyme poly(ADP-ribose) polymerase-1 (PARP-1). Mild damage of DNA activates the DNA repair machinery. In contrast, once excessive oxidative and nitrosative stress-induced DNA damage occurs, like in various forms of myocardial reperfusion injury and heart failure, overactivated PARP initiates an energy-consuming cycle by transferring ADP-ribose units from nicotinamide adenine dinucleotide $\left(\mathrm{NAD}^{+}\right)$to nuclear proteins, resulting in rapid depletion of the intracellular $\mathrm{NAD}^{+}$and ATP pools, slowing the rate of glycolysis and mitochondrial respiration, eventually leading to cellular dysfunction and death. Poly(ADP-ribose) glycohydrolase (PARG) degrades poly(ADP-ribose) (PAR) polymers, generating free PAR polymer and ADP-ribose. Overactivated PARP also facilitates the expression of a variety of inflammatory genes leading to increased inflammation and associated oxidative stress, thus facilitating the progression of cardiovascular dysfunction and heart failure. 
A

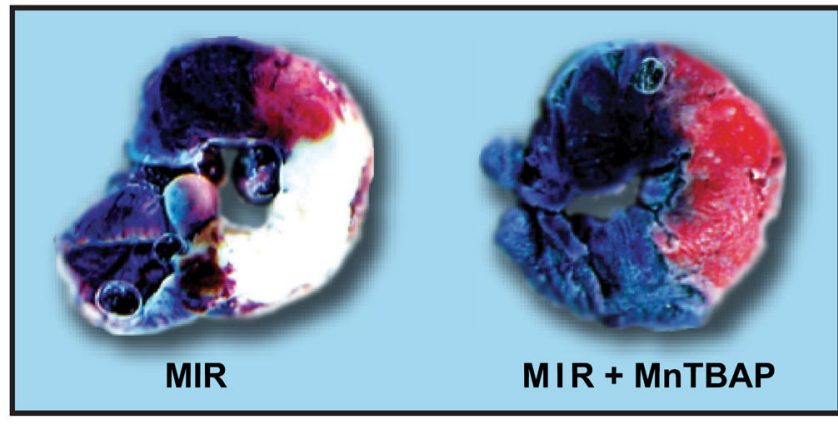

Infarct size ( $\%$ area at risk)

B

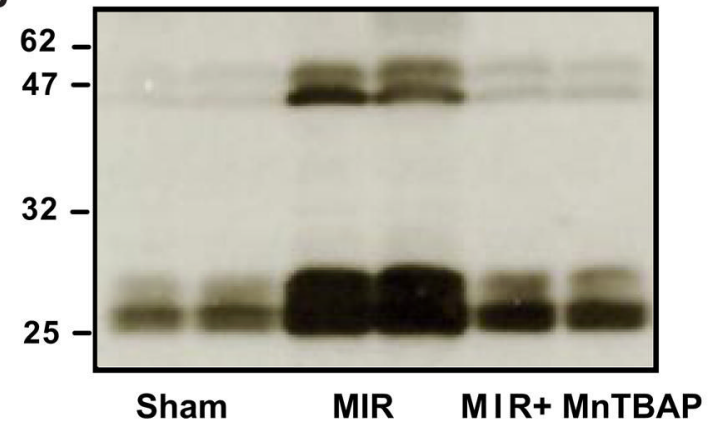

Myocardial 3-NT (fold increase)

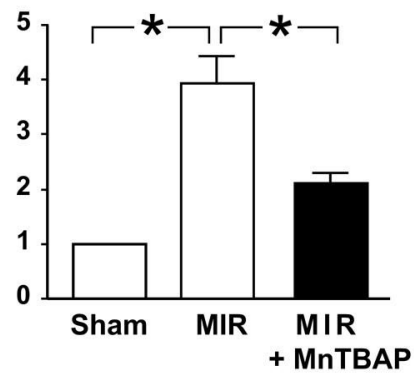

FIG. 10.

Role of peroxynitrite in myocardial infarction. Peroxynitrite scavenger MnTBAP reduces infart size $(A)$ and suppresses myocardial 3-nitrotyrosine formation $(B)$ in rat. [From Levrand et al. (750) with permission from Elsevier.] 

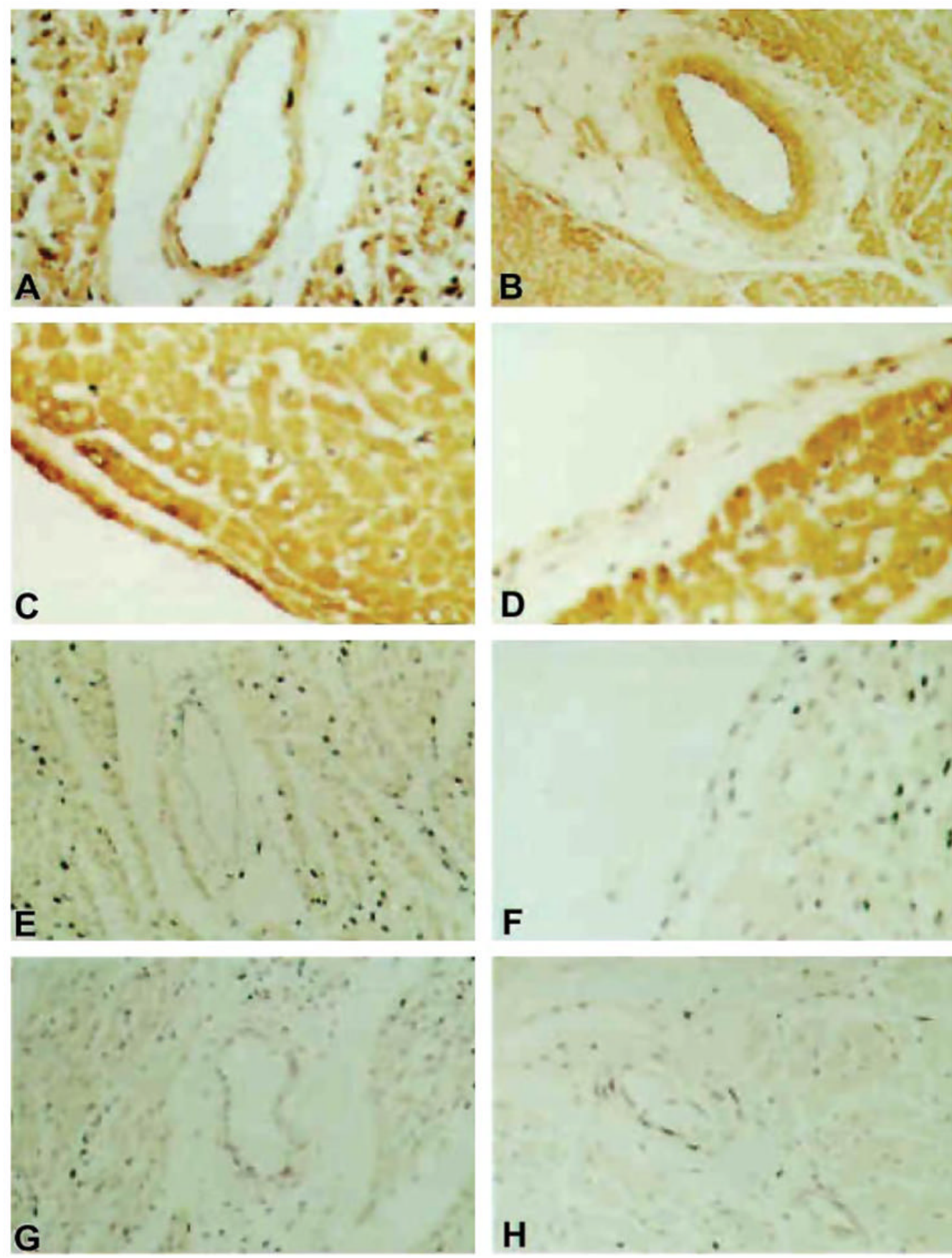

FIG. 11.

Evidence for nitrotyrosine formation in human myocardial inflammation. Representative examples are shown of nitrotyrosine immunoreactivity in cardiac tissue samples from patients with myocarditis, sepsis, or no cardiac disease (control patients). A: low-power $(\times 20)$ photomicrograph of nitrotyrosine immunoreactivity (brown staining) in the myocardium, vascular endothelium, and vascular smooth muscle of myocarditis patient. $B$ : low-power $(\times 20)$ photomicrograph of nitrotyrosine immunoreactivity in the myocardium, vascular endothelium, and vascular smooth muscle of sepsis patient. Note the relative absence of staining of the connective tissue elements. $C$ : higher-power $(\times 40)$ photomicrograph of intense nitrotyrosine immunoreactivity in the endocardium of myocarditis patient. $D$ : higher-power $(\times 40)$ 
photomicrograph of nitrotyrosine immunoreactivity in the endocardium of sepsis patient. $E$ : low-power $(\times 20)$ photomicrograph of minimal nitrotyrosine immunoreactivity in the myocardium and the virtual absence of nitrotyrosine immunoreactivity in the vascular endothelium and vascular smooth muscle of control patient 1C. F: higher-power $(\times 40)$ photomicrograph of nitrotyrosine immunoreactivity in the endocardium of control patient. $G$ : low-power $(\times 20)$ photomicrograph demonstrating the inhibition of nitrotyrosine immunoreactivity by the preincubation of the primary antibody with $10 \mathrm{mM}$ nitrotyrosine before tissue staining in myocarditis patient. $H$ : low-power $(\times 20)$ photomicrograph demonstrating the inhibition of nitrotyrosine immunoreactivity by the preincubation of the primary antibody with $10 \mathrm{mM}$ nitrotyrosine before tissue staining in sepsis patient. [From Kooy et al. (707) with permission from Lippincott Williams \& Wilkins.] 


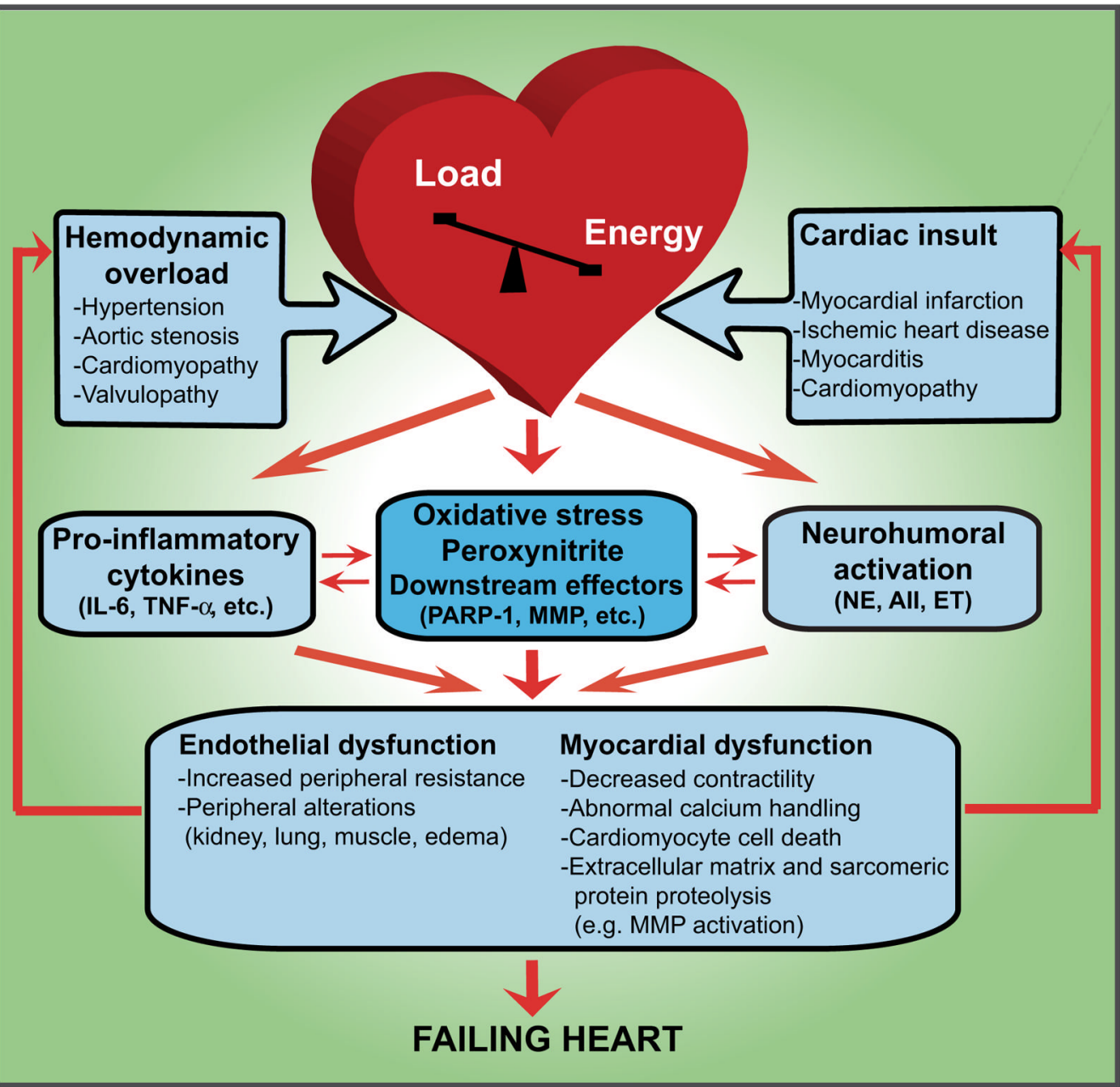

FIG. 12.

Progression of heart failure and the role of oxidative stress and peroxynitrite. The mechanisms leading to heart failure are of multiple origins and include acute and chronic ischemic heart disease, cardiomyopathies, myocarditis, and pressure overload just to mention a few. These diseases result in mismatch between the load applied to the heart and the energy needed for contraction, leading to mechanoenergic uncoupling. After initial insult, secondary mediators such as angiotensin II (AII), norepinephrine (NE), endothelin (ET), proinflammatory cytokines [e.g., tumor necrosis factor- $\alpha$ (TNF- $\alpha$ ) and interleukin 6 (IL-6), in concert with oxidative stress and peroxynitrite, activate downstream effectors (e.g., PARP-1 or MMPs)], act directly on the myocardium or indirectly via changes in hemodynamic loading conditions to cause endothelial and myocardial dysfunction, cardiac and vascular remodeling with hypertrophy, fibrosis, cardiac dilation, and myocardial necrosis, leading eventually to heart failure. The adverse remodeling and increased peripheral resistance further aggravate heart failure. MMPs, matrix metalloproteinases; PARP-1, poly(ADP-ribose) polymerase. [Derived from Pacher et al. (995) with permission from Elsevier.] 

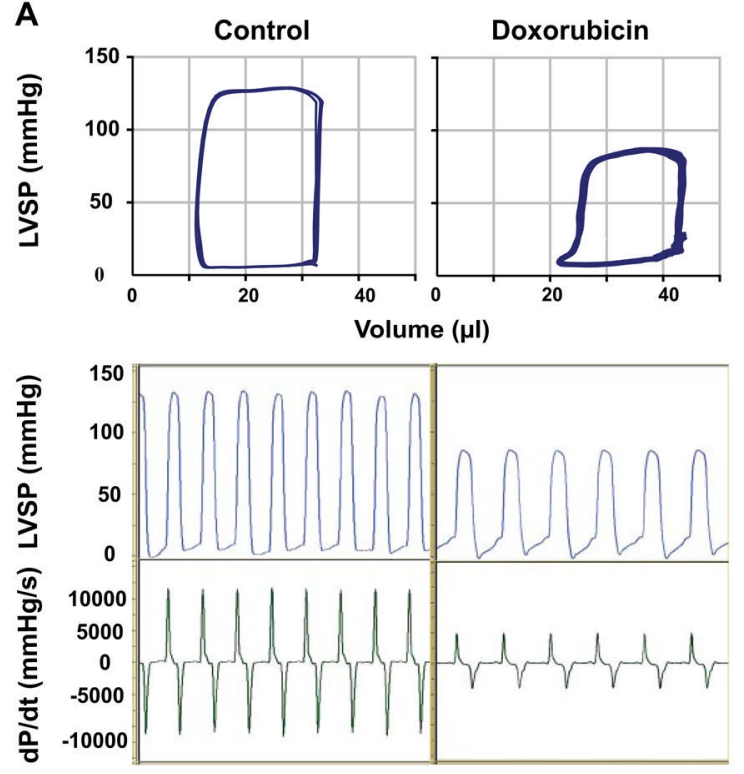
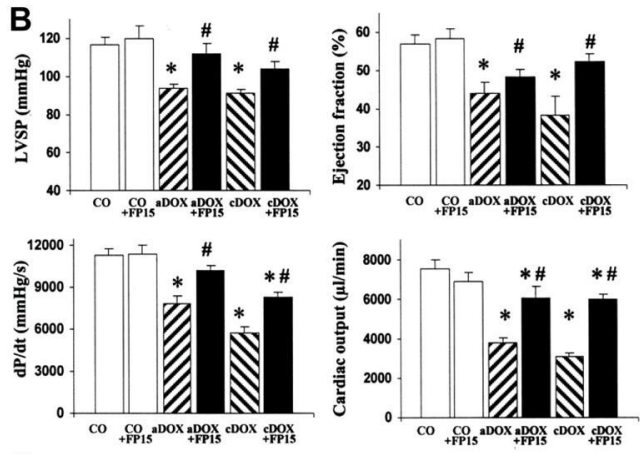

C

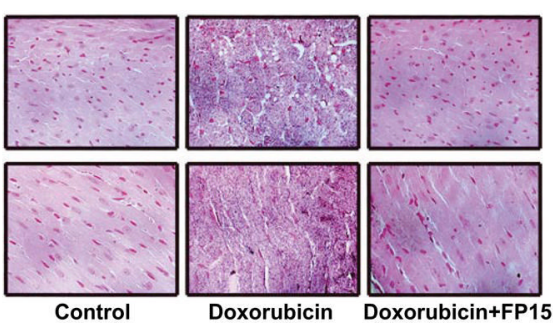

FIG. 13.

Role of peroxynitrite in doxorubicin (DOX)-induced heart failure. $A$ : evidence of severe cardiac dysfunction 5 days after DOX injection in mice. Representative PV loops (top) and left ventricular pressure signal (bottom) from control and DOX and DOX + INO-1001-treated mice. Please note that the rightward shift of PV loops in DOX-treated animals, the decrease of maximal left ventricular pressure, and $+\mathrm{dP} / \mathrm{d} t$ indicate depressed cardiac contractility. $B$ : peroxynitrite scavenger FP15 (black bars) attenuates DOX-induced (hatched bars) acute (aDOX; single dose of $25 \mathrm{mg} / \mathrm{kg}$ ip) and chronic (cDOX; 3 doses of $9 \mathrm{mg} / \mathrm{kg}$ ip every 10th day for 25 days) cardiac dysfunction. Hemodynamic parameters were measured 5 (aDOX) or 25 (cDOX) days after DOX administration. Results are means \pm SE of 10-14 experiments in each group. $* P<0.05$ vs. CO. $\# P<0.05$ vs. aDOX or cDOX. $C$ : evidence of increased myocardial nitrotyrosine formation (widespread dark brown staining) 5 days after DOX injection in mice and reduction by FP15. [From Pacher and co-workers $(985,988)$ with permission from Lippincott Williams \& Wilkins and Prof. Demetrios A. Spandidos.] 


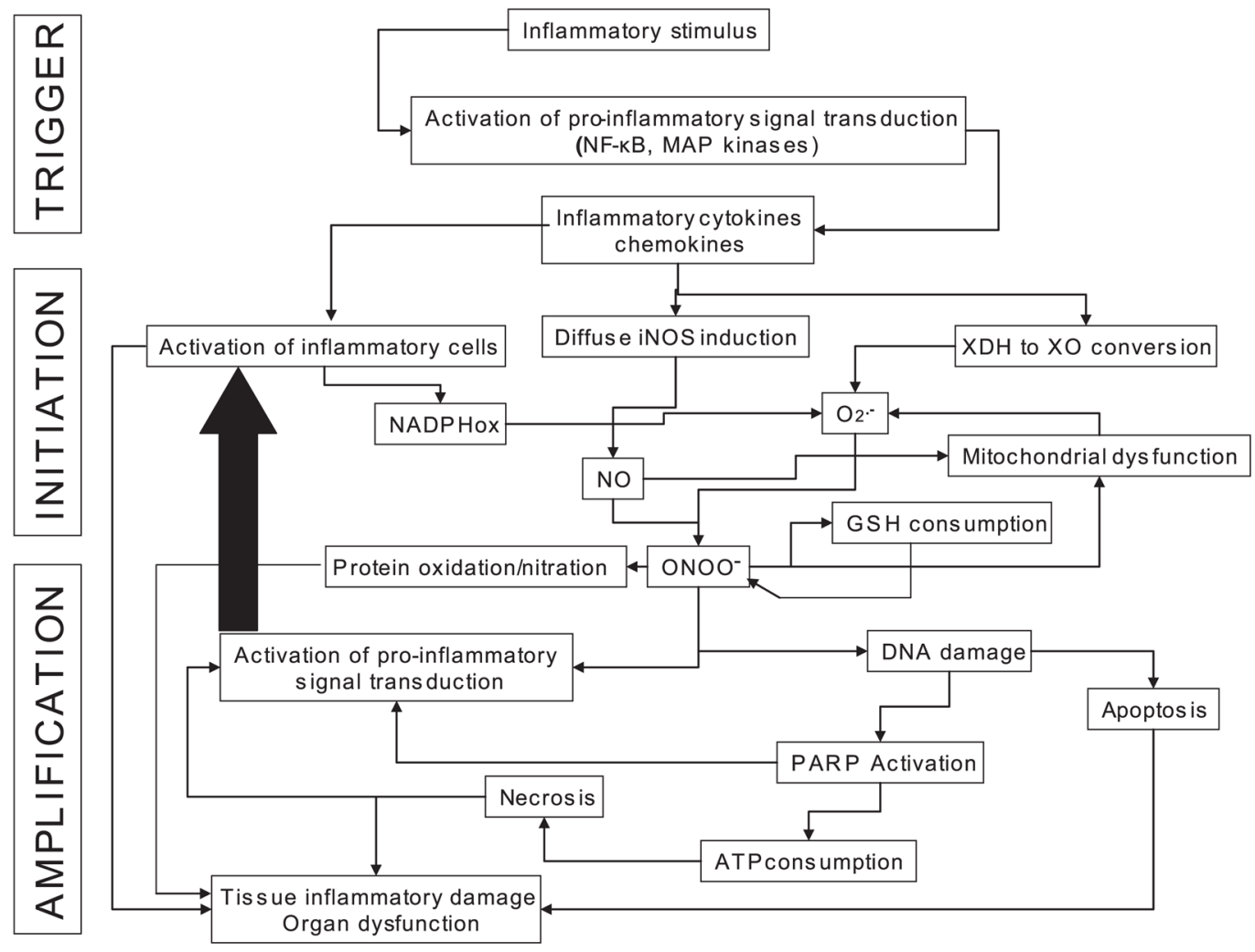

FIG. 14.

Mechanisms of amplification of inflammation by peroxynitrite. Inflammation is triggered by the activation of multiple signaling cascades culminating in the upregulated production of an array of proinflammatory cytokines and chemokines. Those initiate a more complex inflammatory reaction characterized by the activation of inflammatory cells and the stimulated activity of enzymes, including inducible NO synthase (iNOS), which produces high amounts of NO, and the superoxide $\left(\mathrm{O}_{2}{ }^{--}\right)$-producing enzymes NADPH oxidase (NADPHox) and xanthine oxidase (XO). The simultaneous production of $\mathrm{NO}$ and $\mathrm{O}_{2}{ }^{--}$results in the generation of peroxynitrite $\left(\mathrm{ONOO}^{-}\right)$, which in turn damages target molecules including proteins, glutathione (GSH), mitochondria, and DNA. DNA damage can initiate apoptotic cell death and is also the obligatory trigger for the activation of poly(ADP-ribose) polymerase (PARP), which may induce cell necrosis by ATP depletion. Both $\mathrm{ONOO}^{-}$and PARP further participate to the upregulation of proinflammatory signal transduction pathways, thereby producing a selfamplifying cycle of inflammatory cell injury, as indicated by the black arrow. 


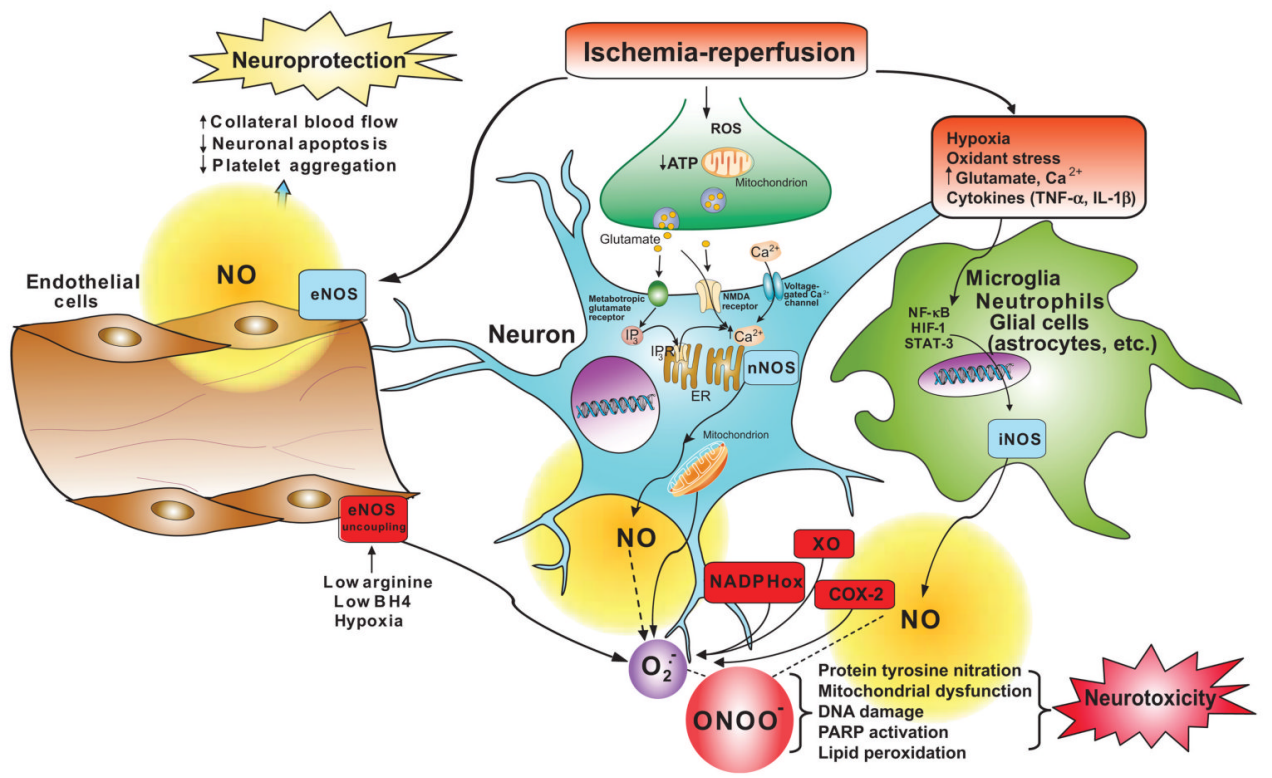

FIG. 15.

Roles of NO and peroxynitrite in the pathophysiology of stroke. Brain ischemia and reperfusion leads to transient stimulation of the activity of endothelial NO synthase (eNOS), resulting in brief increases in endothelial NO generation, associated with neuroprotective actions in stroke. In parallel, ischemic energy depletion and oxidant (ROS) production triggers the release of glutamate, which results in neuronal calcium overload from extracellular (activation of calcium channels) and intracellular (phosphoinositol-3-kinase-endoplasmic reticulum signaling) sources. Calcium overload results in prolonged synthesis of NO, due to stimulated activity of the neuronal isoform of NO synthase (nNOS). Enhanced NO generation also depends on the induced expression of inducible NOS (iNOS) in various types of reactive inflammatory cells, upon the activation of several cell signaling pathways (HIF-1, STAT-3, and NF $\kappa$ B) in response to hypoxia, cytokines, oxidants, and glutamate. During the same period of time, superoxide production is enhanced due to uncoupling of eNOS, mitochondrial dysfunction, and the stimulated activity of NADPH oxidase, xanthine oxidase, and cyclooxygnease-2 (COX-2). Formation of peroxynitrite is then markedly favored, damaging lipids, proteins, DNA, and triggering the activation of poly(ADP-ribose) polymerase (PARP), which all contribute significantly to neurotoxicity in stroke. 

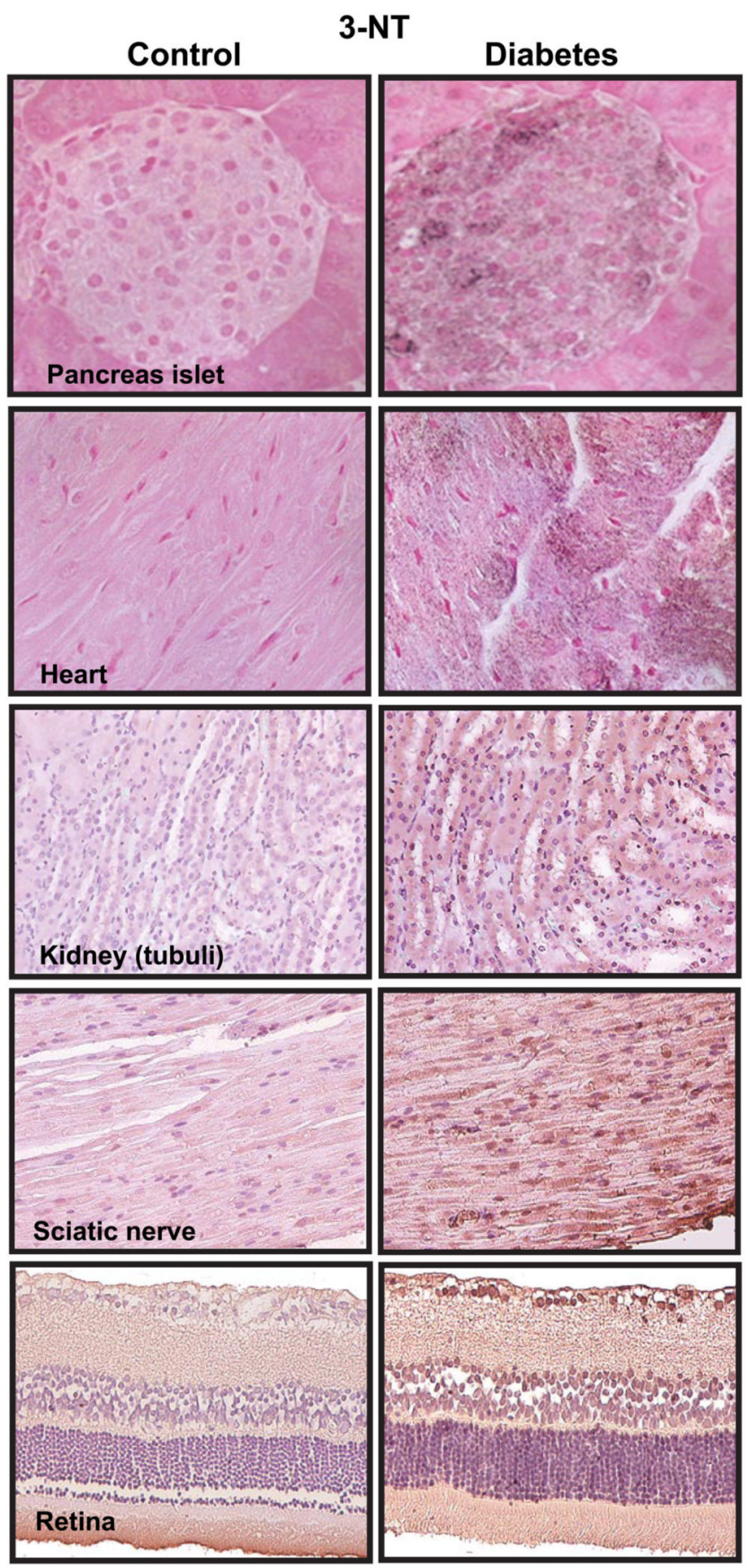

FIG. 16.

Evidence for nitrotyrosine formation from various tissues of diabetic mice and rats. Immunohistochemical staining for nitrotyrosine (dark brown staining) from control (left) and diabetic (right) murine tissues. [Derived from Pacher et al. (994) and Szabo et al. (1234), with permissions from The Feinstein Institute for Medical Research and Bentham Science Publishers.] 


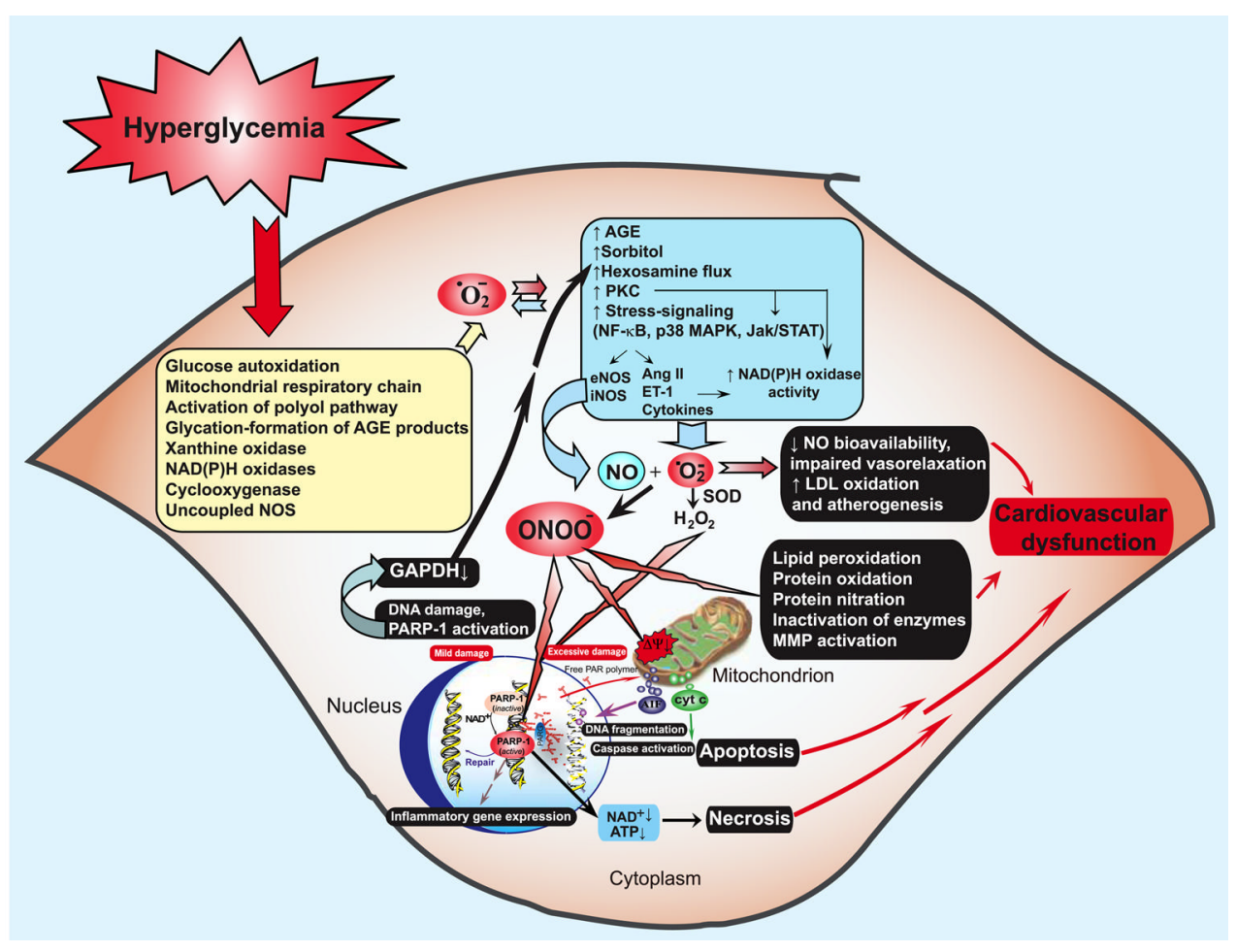

FIG. 17.

Mechanisms of cardiovascular dysfunction in diabetes: role of superoxide and peroxynitrite. Hyperglycemia induces increased superoxide anion $\left(\mathrm{O}_{2}{ }^{-}\right)$production via activation of multiple pathways including xanthine and NAD $(\mathrm{P}) \mathrm{H}$ oxidases, cyclooxygenase, uncoupled nitric oxide synthase (NOS), glucose autoxidation, mitochondrial respiratory chain, polyol pathway, and formation of advanced glycation end products (AGE). Superoxide activates AGE, protein kinase $\mathrm{C}$ (PKC), polyol (sorbitol), hexosamine, and stress-signaling pathways leading to increased expression of inflammatory cytokines, angiotensin II (Ang II), endothelin-1 (ET-1), and NAD(P)H oxidases, which in turn generate more superoxide via multiple mechanisms. Hyperglycemia-induced increased superoxide generation may also favor an increased expression of nitric oxide synthases (NOS) through the activation of $\mathrm{NF} \kappa \mathrm{B}$, which may increase the generation of nitric oxide (NO). Superoxide anion may quench $\mathrm{NO}$, thereby reducing the efficacy of a potent endothelium-derived vasodilator system. Superoxide can also be converted to hydrogen peroxide $\left(\mathrm{H}_{2} \mathrm{O}_{2}\right)$ by superoxide dismutase (SOD) and interact with $\mathrm{NO}$ to form a reactive oxidant peroxynitrite $\left(\mathrm{ONOO}^{-}\right)$, which induces cell damage via lipid peroxidation, inactivation of enzymes and other proteins by oxidation and nitration, and activation of matrix metalloproteinases (MMPs) among others. Peroxynitrite also acts on mitochondria [decreasing the membrane potential $(\Psi)$ ], triggering the release of proapoptotic factors such as cytochrome $c(\mathrm{Cyt} c)$ and apoptosis-inducing factor (AIF). These factors mediate caspase-dependent and caspase-independent apoptotic death pathways. Peroxynitrite, in concert with other oxidants (e.g., $\mathrm{H}_{2} \mathrm{O}_{2}$ ), causes strand breaks in DNA, activating the nuclear enzyme poly(ADP-ribose) polymerase-1 (PARP-1). Mild damage to DNA activates the DNA repair machinery. In contrast, once excessive oxidative and nitrosative stress-induced DNA damage occurs, overactivated PARP-1 initiates an energy-consuming cycle by transferring ADP-ribose units (small red spheres) from $\mathrm{NAD}^{+}$to nuclear proteins, resulting in rapid depletion of the intracellular $\mathrm{NAD}^{+}$and $\mathrm{ATP}$ pools, slowing the rate of glycolysis and mitochondrial respiration, and eventually leading to cellular dysfunction and death. Poly(ADP-ribose) glycohydrolase (PARG) degrades poly(ADP-ribose) (PAR) polymers, generating free $\mathrm{PAR}$ polymer and ADP-ribose, which may signal to the mitochondria 
to induce AIF release. PARP-1 activation also leads to the inhibition of cellular glyceraldehyde-3-phosphate dehydrogenase (GAPDH) activity, which in turn favors the activation of PKC, AGE, and hexosamine pathway leading to increased superoxide generation. PARP-1 also regulates the expression of a variety of inflammatory mediators, which might facilitate the progression of diabetic cardiovascular complications. [From Pacher and Szabo (996), with permission from Elsevier.] 


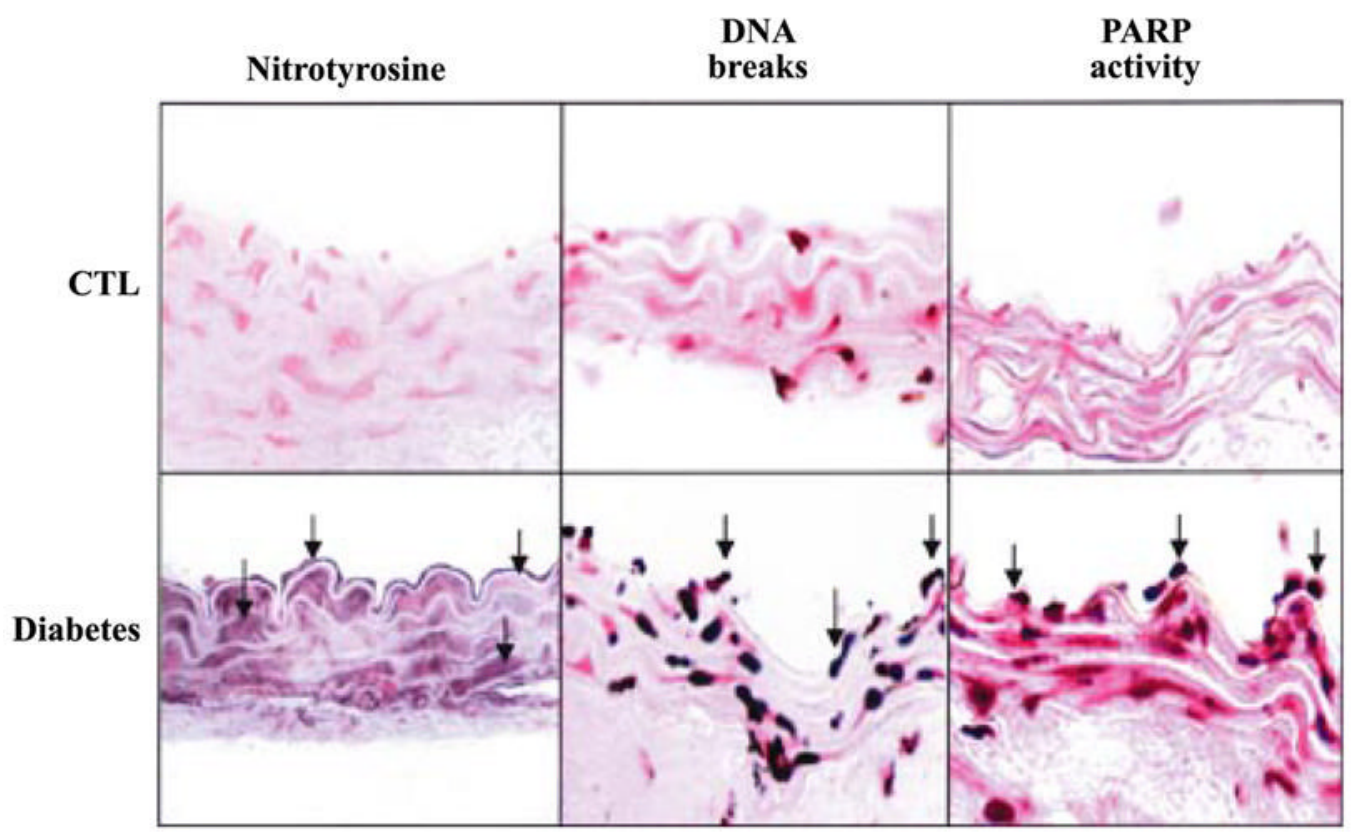

FIG. 18.

Evidence for nitrotyrosine formation and PARP activation in diabetic vasculature. Immunohistochemical staining for nitrotyrosine, terminal deoxyribonucleotidyl transferasemediated dUTP nick-end labeling (indicator of DNA breaks), and poly-(ADP-ribose) (index of PARP activity) in control rings (top row) and in rings from diabetic mice (bottom row). [Derived from Garcia Soriano et al. (430) with permission from Nature Publishing Group.] 
PACHER et al.

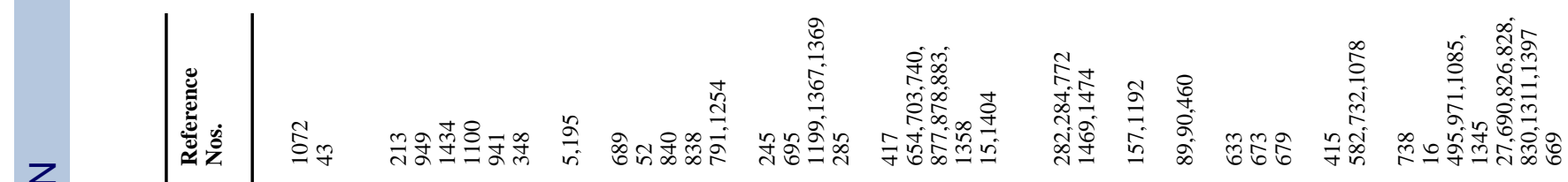
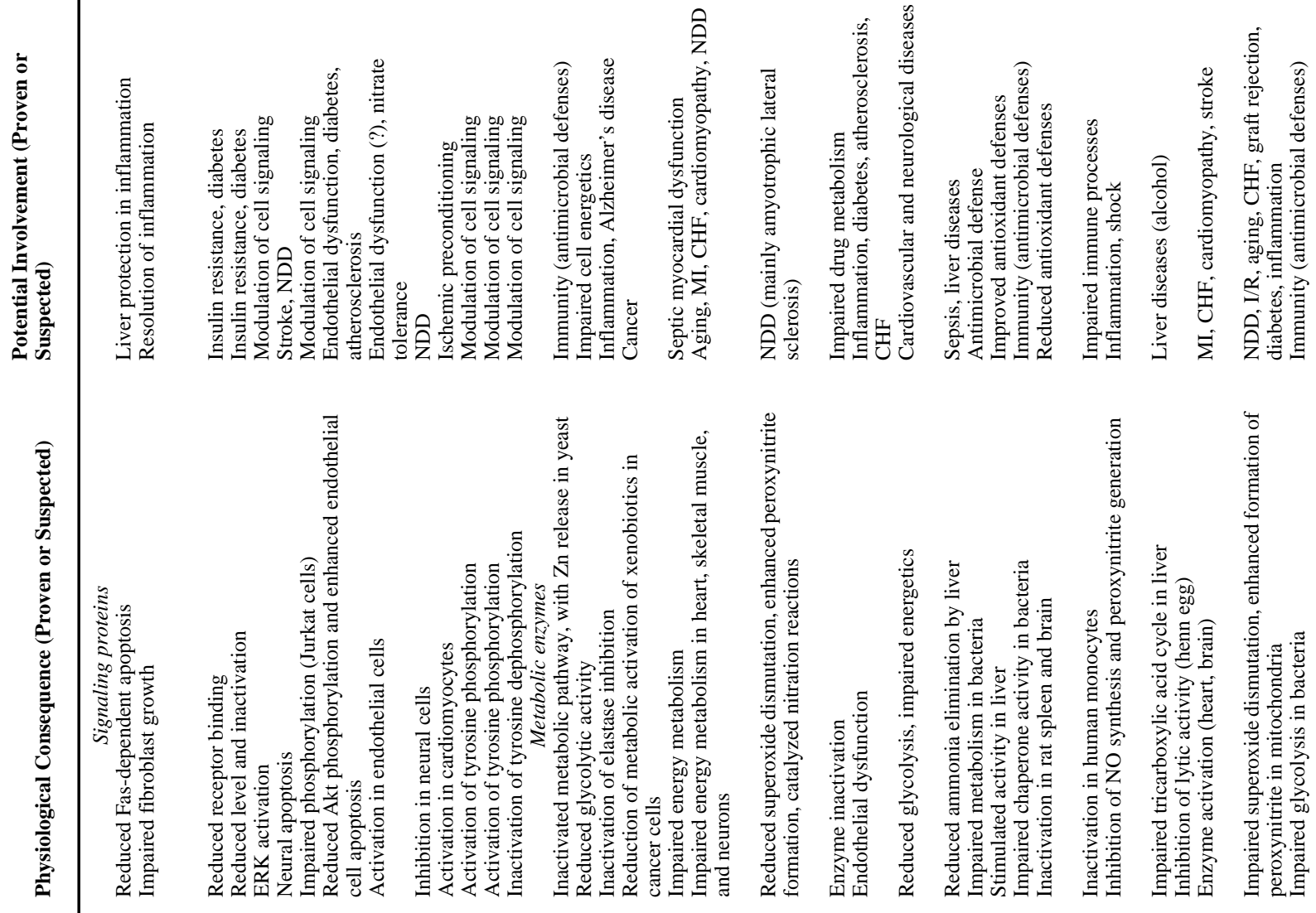

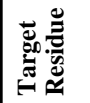

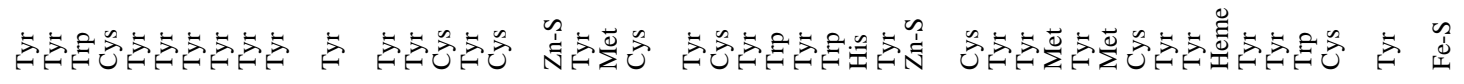

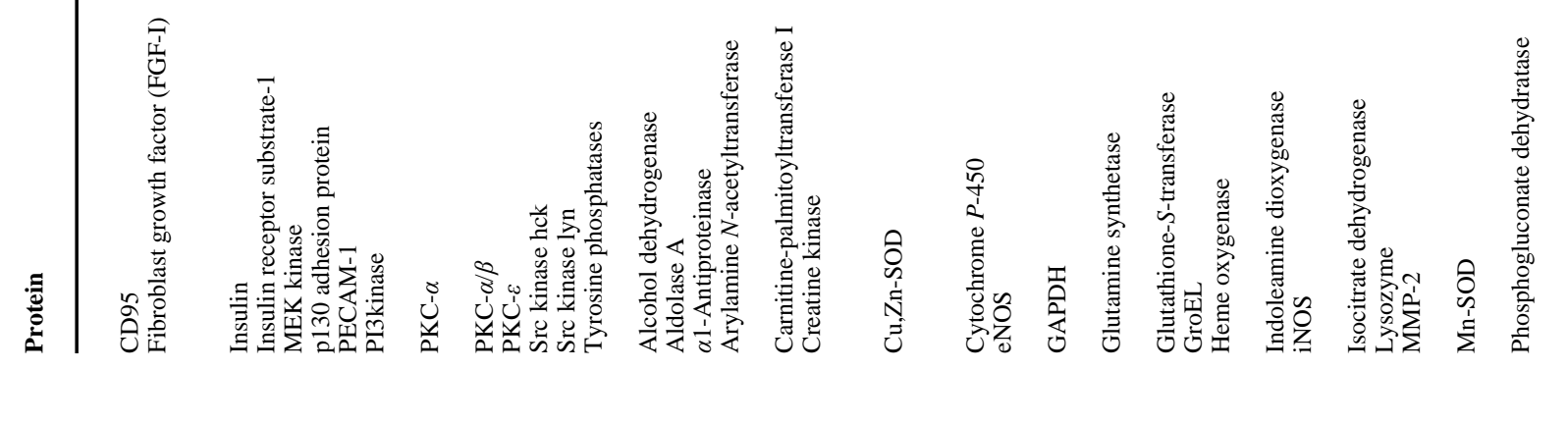

Physiol Rev. Author manuscript; available in PMC 2008 February 20. 
PACHER et al.

Page 175

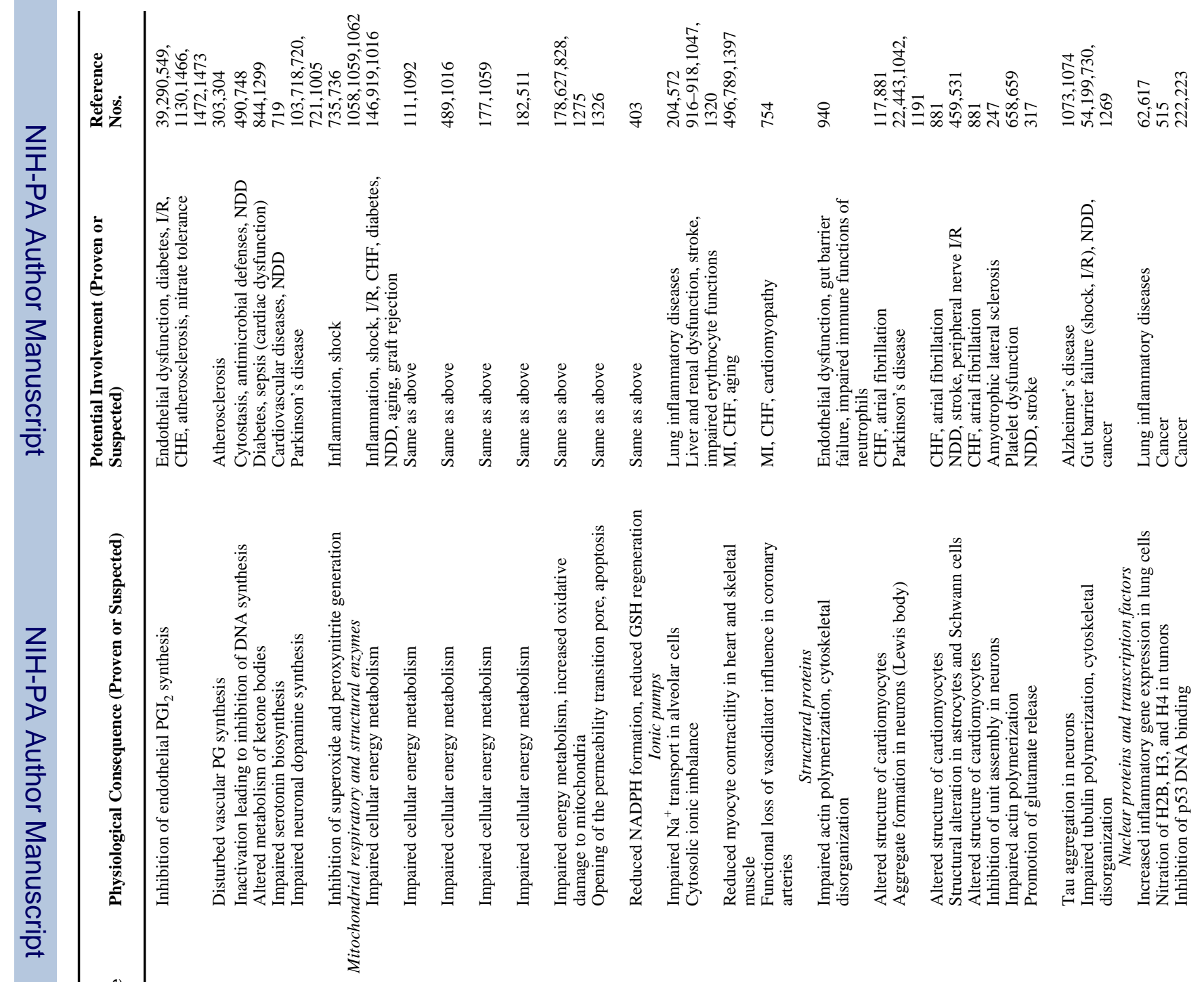

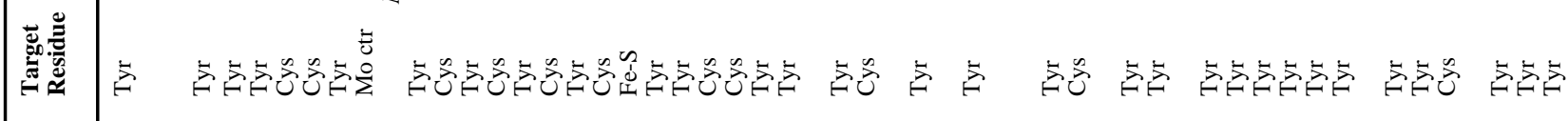

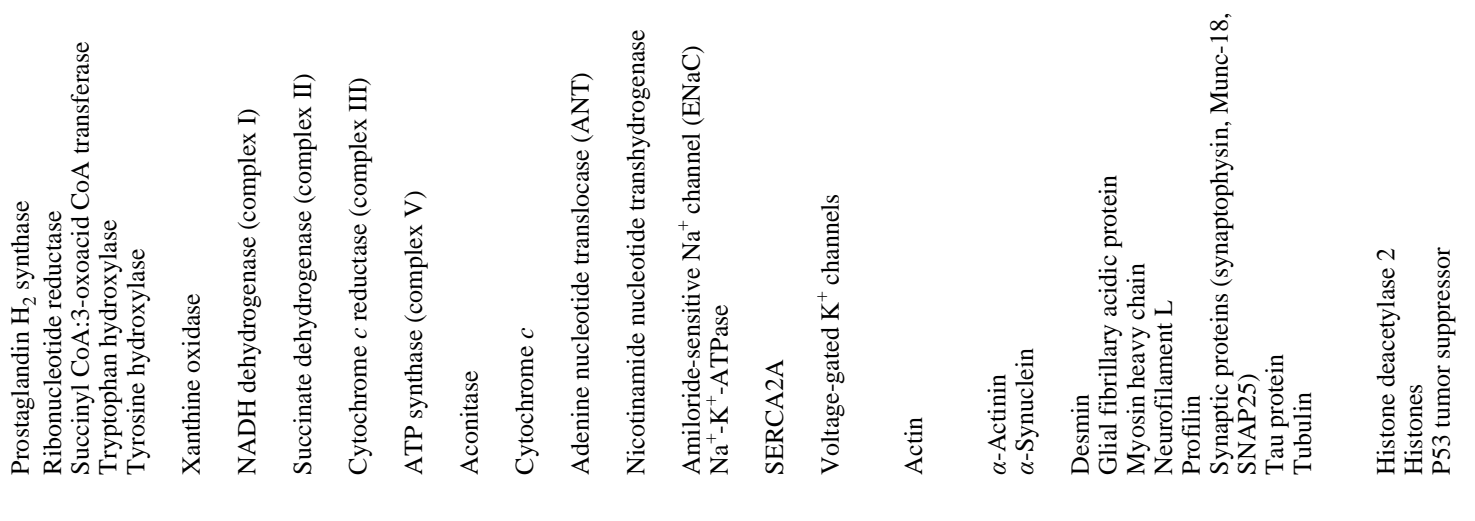


PACHER et al.

Page 176

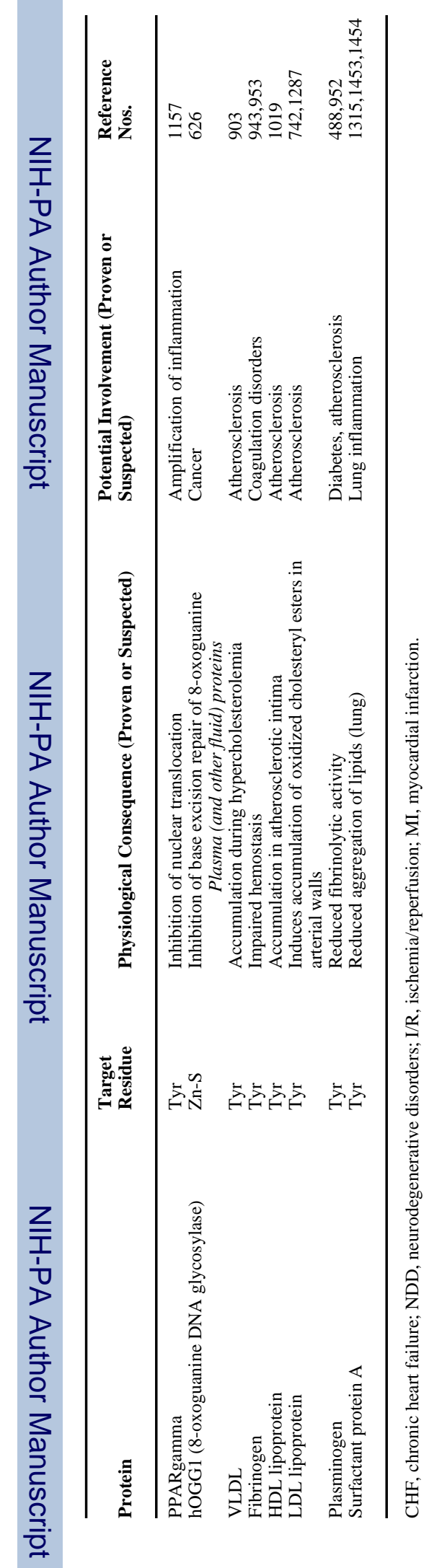

Physiol Rev. Author manuscript; available in PMC 2008 February 20. 
TABLE 2

Major signaling pathways shown to be influenced by peroxynitrite in vitro

\begin{tabular}{|c|c|c|c|}
\hline Signaling Protein & $\begin{array}{l}\text { Mode of Action of } \\
\text { Peroxynitrite } \\
\text { (Activation vs. } \\
\text { Inhibition) }\end{array}$ & Cell Type & Reference Nos. \\
\hline EGFR & A & Fibroblasts, myofibroblasts & 687,1434 \\
\hline EGFR & $\mathrm{I}$ & A431, Caco-2B cells & 1301,1316 \\
\hline PDGFR & A & Fibroblasts & 687 \\
\hline TrkB & A & Fibroblasts & 1427 \\
\hline CD95 & $\mathrm{I}$ & Hepatocytes & 1072 \\
\hline Src & A & $\begin{array}{l}\text { RBCs, astrocytes, synaptososmes, SH-SY5Y, } \\
\text { endothelial cells }\end{array}$ & $\begin{array}{l}519,638,760,829,836-838,840, \\
1470\end{array}$ \\
\hline ERK & A & $\begin{array}{l}\text { Fibroblasts, neutrophils, cardiomyocytes, } \\
\text { VSMCs, endothelial, neural cells }\end{array}$ & $\begin{array}{l}57,191,638,645,735,1024,1099, \\
1307,1434,1477\end{array}$ \\
\hline JNK & A & $\begin{array}{l}\text { Alveolar, endothelial, bronchial cells, } \\
\text { cardiomyocytes, fibroblasts }\end{array}$ & $23,450,922,1024,1128,1162$ \\
\hline p38 & A & $\begin{array}{l}\text { Endothelial, bronchial, neural cells, VSMCs, } \\
\text { cardiomyocytes, hepatocytes }\end{array}$ & $\begin{array}{l}120,354,414,450,638,922,965 \\
1024,1120,1128,1307,1440\end{array}$ \\
\hline PKC & A & Cardiomyocytes, endothelial cells & 52,195 \\
\hline $\mathrm{PKC}$ & $\mathrm{I}$ & Neural cells & 689 \\
\hline $\mathrm{PI} 3 \mathrm{~K} / \mathrm{PKB}$ & A & Hepatocytes, fibroblasts, endothelial cells & $652,687,1133$ \\
\hline $\mathrm{PI} 3 \mathrm{~K} / \mathrm{PKB}$ & $\mathrm{I}$ & $\begin{array}{l}\text { Macrophages, adipocytes, PC12, endothelial, } \\
\text { retinal cells }\end{array}$ & $348,536,949,1193,1471$ \\
\hline $\mathrm{NF} \kappa \mathrm{B}$ & A & Neutrophils, monocytes, L8 rat myoblasts & $58,391,640,674,851,1477$ \\
\hline $\mathrm{NF} \kappa \mathrm{B}$ & $\mathrm{I}$ & $\begin{array}{l}\text { Cardiomyocytes, EAHY-926, HMEC-I, } \\
\text { SHSY-5Y, HEK293, P19 cells }\end{array}$ & 749,1006 \\
\hline
\end{tabular}

A, activation; I, inhibition; NFאB, nuclear factor kappa B; PKC, protein kinase C; PI3K, phosphatidylinositol 3-kinase; ERK, extracellular-regulated kinase; JNK, Janus kinase; EGFR, epidermal growth factor receptor; PDGFR, platelet-derived growth factor receptor; TrkB, truncated tyrosine protein kinase. 
TABLE 3

Selected effects of peroxynitrite with potential relevance to cardiovascular pathophysiology

\begin{tabular}{|c|c|}
\hline Effect(s) & Reference Nos. \\
\hline Inhibition of $\mathrm{BH}_{4}$-dependent, NAD-dependent and antioxidant enzymes & Reviewed in 1227 \\
\hline Depletion of antioxidants (e.g., glutathione, cysteine) & Reviewed in 1227 \\
\hline Increased lipid peroxidation & Reviewed in 1227 \\
\hline $\begin{array}{l}\text { Impairment of mitochondrial respiration and energy metabolism (inactivation of cytochromes, NADH- } \\
\text { COQ1, etc.) }\end{array}$ & $144,740,1380$ \\
\hline Promotion of $\mathrm{Ca}^{2+}$-dependent mitochondrial permeability transition pore opening & $142,999,1000$ \\
\hline Inhibition of myocardial aconitase & 211 \\
\hline Impairment of $\alpha_{1^{-}}, \beta_{1^{-}}$, and $\beta_{2}$-adrenoceptors, vasopressin VIA and ANG II receptor function & $84,704-706,751,752$ \\
\hline Inactivation of myofibrillar creatine kinase and impairment of contractile function & $614,877,882$ \\
\hline Inactivation of $\alpha$-actinin in cardiomyocytes & 117 \\
\hline $\begin{array}{l}\text { Inactivation of sarcoplasmic reticulum } \mathrm{Ca}^{2+} \text {-ATPase in myocytes and vascular smooth muscle, } \mathrm{Ca}^{2+} \\
\text { dysregulation }\end{array}$ & $483,789,1329$ \\
\hline Activation of myocardial matrix metalloproteinases ( $S$-glutoxidation of prometalloproteinases) & 1345 , reviewed in 995 \\
\hline $\begin{array}{l}\text { Alterations in signaling (activation of extracellular signal-related kinase; activation or inhibition of } \\
\mathrm{NF} \kappa \mathrm{B} \text {; nitration of } \mathrm{PKC}-\varepsilon \text {-promoting translocation and activation) }\end{array}$ & $52,749,1024,1178$ \\
\hline Activation or inactivation of cardiovascular $\mathrm{K}^{+} \mathrm{ATP}$ channel(s) & $83,471,473,783$ \\
\hline Inactivation voltage-gated $\mathrm{K}^{+} \mathrm{K}(\mathrm{v})$ and $\mathrm{Ca}^{2+}$-activated $\mathrm{K}^{+}$channels in coronary arterioles & $754,785,786$ \\
\hline Impairment of vascular relaxation by various mechanisms & $83,469,471,473,705,879$ \\
\hline Inactivation of vascular prostacyclin synthase & $228,290,1466,1467,1472,1475$ \\
\hline Uncoupling of NO synthase leading to production of superoxide rather than NO & $405-407,734,889$ \\
\hline Cardiac ion channel modulation?, arrhythmia & 1179,1268 \\
\hline $\begin{array}{l}\text { Upregulation of adhesion molecules in endothelial cells, endothelial glycocalyx disruption, enhanced } \\
\text { neutrophils adhesion (presumably via NF } \kappa \text { B activation) }\end{array}$ & $424,723,1178,1447,1477$ \\
\hline Pro- or antiaggregatory effects on platelets depending on the environment & 908,953; reviewed in 1097 \\
\hline $\begin{array}{l}\text { Apoptosis in cardiomyocytes, vascular endothelial and smooth muscle cells as a consequence of } \\
\text { mitochondrial injury, DNA injury, caspase activation, signal transduction disturbances, calcium } \\
\text { dysregulation }\end{array}$ & $30,319,480,750,755,756,879$ \\
\hline $\begin{array}{l}\text { DNA damage, PARP activation, cell necrosis in cardiomyocytes, vascular endothelial and smooth } \\
\text { muscle cells as a consequence of mitochondrial injury, energetic collapse, oxidation, nitration, } \\
\text { antioxidant depletion, calcium dysregulation }\end{array}$ & $\begin{array}{l}444,1233,1243,1244,1450 ; \text { reviewed in } \\
995,1230,1235\end{array}$ \\
\hline
\end{tabular}

ANG II, angiotensin II; $\mathrm{BH}_{4}$, tetrahydrobiopterin; $\mathrm{NF} \kappa \mathrm{B}$, nuclear factor kappa B; PARP, poly(ADP-ribose) polymerase; NO, nitric oxide. 
TABLE 4

Evidence implicating endogenous peroxynitrite formation and/or protein nitration in cardiac and vascular diseases

\begin{tabular}{|c|c|c|c|}
\hline Disease/Trigger & Experimental Model & Main Findings & Reference Nos. \\
\hline \multicolumn{4}{|c|}{ Myocardial ischemia reperfusion $(I / R)$} \\
\hline Global I/R & Rat heart & $\begin{array}{l}\text { Cardiac dysfunction, decreased efficiency } \\
\text { of increased } \mathrm{O}_{2} \text { utilization, iNOS } \\
\text { expression, increased superoxide and } \\
\text { myocardial peroxynitrite/nitrotyrosine } \\
\text { (NT) formation in hearts subjected to I/R. } \\
\text { NOS or MMP inhibitors, superoxide } \\
\text { dismutase, glutathione or urate greatly } \\
\text { enhance the recovery of contractile } \\
\text { function in postischemic hearts and } \\
\text { reduced myocardial nitrotyrosine } \\
\text { formation. Urate or NOS inhibitor nitro-L- } \\
\text { arginine also improves efficiency of } \mathrm{O}_{2} \\
\text { utilization. }\end{array}$ & $\begin{array}{l}385,429,740,781 \\
816,1344,1345 \\
1398,1413,1478\end{array}$ \\
\hline Regional I/R & Rat & $\begin{array}{l}\text { Peroxynitrite decomposition catalysts } \\
\text { FP- } 15 \text { and MnTBAP reduce myocardial } \\
\text { infarct size and myocyte apoptosis. }\end{array}$ & 750,985 \\
\hline $\begin{array}{l}\text { I/R associated with acute cardiac } \\
\text { transplantation }\end{array}$ & Rat & $\begin{array}{l}\text { Peroxynitrite decomposition catalyst } \\
\text { WW85 reduces lipid peroxidation, NT } \\
\text { formation, PARP activation in grafts, } \\
\text { improves function, and prolongs survival. }\end{array}$ & 1027 \\
\hline Regional I/R & Dog & $\begin{array}{l}\text { Intracoronary administration of L-arginine } \\
\text { or infusion of NO donor SNAP during I/R } \\
\text { aggravates myocardial stunning following } \\
\text { I/R through increased NT formation in the } \\
\text { myocardium of dogs, which is improved by } \\
\text { NOS inhibition. }\end{array}$ & $902,1437,1438$ \\
\hline Regional or repetitive regional I/R & Pig & $\begin{array}{l}\text { Increased nitrotyrosine formation and } \\
\text { cardiac dysfunction following I/R. } \\
\text { Reduction of infarct size, reactive } \\
\text { hyperemia, and myocardial NT formation } \\
\text { by peroxynitrite decomposition catalyst } \\
\text { FP-15. }\end{array}$ & $48,100,573$ \\
\hline Cardiopulmonary bypass surgery & Human & $\begin{array}{l}\text { Increased plasma NT levels, myocardial } \\
\text { NOS expression/activity, and peroxynitrite } \\
\text { formation following myocardial I/R during } \\
\text { open heart surgery. }\end{array}$ & $525,526,864$ \\
\hline Coronary artery disease & Human & $\begin{array}{l}\text { Increased NT formation and cardiac } \\
\text { dysfunction in hibernating myocardium. }\end{array}$ & 47 \\
\hline Coxsackie B3 virus & $\begin{array}{l}\text {, cardiac allograft rejec } \\
\text { Mouse }\end{array}$ & $\begin{array}{l}\text { ransplant coronary artery disease } \\
\text { iNOS expression and myocardial NT } \\
\text { formation correlates with the damage. }\end{array}$ & 96 \\
\hline LPBM5 retrovirus (murine AIDS model) & Mouse & $\begin{array}{l}\text { Progressive cardiac dysfunction correlated } \\
\text { with increased myocardial inflammation } \\
\text { and protein nitration. }\end{array}$ & 201 \\
\hline Porcine cardiac myosin & Mouse, rat & $\begin{array}{l}\text { Autoimmune myocarditis characterized by } \\
\text { myocardial destruction, inflammation, and } \\
\text { increased iNOS expression and } \\
\text { nitrotyrosine formation in inflammatory } \\
\text { macrophages and in cardiomyocytes, } \\
\text { which is attenuated by iNOS inhibitor } \\
\text { aminoguanidine. }\end{array}$ & $38,616,1160$ \\
\hline Mixture of inflammatory cytokines & Mouse heart & $\begin{array}{l}\text { Increased xanthine oxidase- and NADPH } \\
\text { oxidase- dependent superoxide production, } \\
\text { nitrotyrosine formation, and cardiac } \\
\text { dysfunction, which is improved by } \\
\text { superoxide and peroxynitrite scavengers } \\
\text { iron and FeTPPS. }\end{array}$ & 384 \\
\hline Mixture of inflammatory cytokines & Rat heart & $\begin{array}{l}\text { Increased myocardial peroxynitrite } \\
\text { formation and matrix metalloproteinase } 2 \\
\text { activation (MMP-2), LV dysfunction. } \\
\text { MMP-2 antibody, or inhibitors attenuated } \\
\text { the decline in myocardial function. }\end{array}$ & 428 \\
\hline Intracoronary inflammatory cytokine & Dog & $\begin{array}{l}\text { Increased nitrotyrosine formation and } \\
\text { sustained cardiac dysfunction, which is } \\
\text { prevented by iNOS inhibitor } \\
\text { aminoguanidine or superoxide scavenger } \\
\text { OPC-6535. }\end{array}$ & 210,978 \\
\hline Endotoxin & Rat heart, rat & $\begin{array}{l}\text { Enhanced generation of } \mathrm{NO} \text { and superoxide } \\
\text { and peroxynitrite in dysfunctional hearts }\end{array}$ & $\begin{array}{l}265,604,670,729, \\
981\end{array}$ \\
\hline
\end{tabular}




\begin{tabular}{|c|c|c|c|}
\hline Disease/Trigger & Experimental Model & Main Findings & Reference Nos. \\
\hline & & $\begin{array}{l}\text { and aortas from endotoxemic rats. iNOS } \\
\text { inhibitor mercaptoethylguanidine and } \\
\text { peroxynitrite decomposition catalyst } \\
\text { FeTPPS improved contractile function, } \\
\text { endotoxin-induced hypotension, and } \\
\text { decreased myocardial and aortic NT } \\
\text { formation. It also decreased NF } \kappa \text { B } \\
\text { inhibitory protein I } \kappa \text { B degradation and } \\
\text { plasma TNF- } \alpha \text {, and vascular endothelial } \\
\text { cell- leukocyte activation. }\end{array}$ & \\
\hline Viral myocarditis, septic shock & Human heart & $\begin{array}{l}\text { Increased nitrotyrosine immunoreactivity } \\
\text { in myocardial specimens with viral } \\
\text { myocarditis and sepsis. }\end{array}$ & 201,707 \\
\hline Cardiac allograft & $\mathrm{iNOS}^{-/-}$mice heart & $\begin{array}{l}\text { iNOS gene deletion improves graft } \\
\text { function, attenuated myocardial NT } \\
\text { formation and damage. }\end{array}$ & 1248 \\
\hline Cardiac allograft & Rat heart & $\begin{array}{l}\text { Increased iNOS expression and } \\
\text { nitrotyrosine formation during cardiac } \\
\text { allograft rejection correlates with the } \\
\text { damage, and attenuated by iNOS inhibitor } \\
\text { or peroxynitrite decomposition catalyst. }\end{array}$ & 11,1104 \\
\hline $\begin{array}{l}\text { Cardiac allograft, transplant coronary } \\
\text { artery disease }\end{array}$ & Human heart & $\begin{array}{l}\text { Increased iNOS expression and } \\
\text { nitrotyrosine formation in human coronary } \\
\text { arteries of patients with human transplant } \\
\text { coronary artery disease and in human } \\
\text { cardiac allografts following rejection. }\end{array}$ & $\begin{array}{l}342,1027,1069 \\
1249,1371\end{array}$ \\
\hline Cardiac-specific iNOS overexpression & Mouse & $\begin{array}{l}\text { Increased myocardial peroxynitrite } \\
\text { formation, inflammation, cardiac fibrosis, } \\
\text { hypertrophy, and dilatation; increased } \\
\text { sudden cardiac death. }\end{array}$ & 913 \\
\hline Doxorubicin & Mouse & $\begin{array}{l}\text { Increased myocardial iNOS expression and } \\
\text { nitrotyrosine formation, which correlates } \\
\text { with cardiac dysfunction. }\end{array}$ & 883,1355 \\
\hline Doxorubicin & Mouse & $\begin{array}{l}\text { Severe LV dysfunction and increased } \\
\text { myocardial nitrotyrosine formation, matrix } \\
\text { metalloproteinase activation in acute and } \\
\text { chronic models of doxorubicin- induced } \\
\text { heart failure, which are improved by } \\
\text { peroxynitrite decomposition catalyst, } \\
\text { FP-15. }\end{array}$ & 44,985 \\
\hline Chronic myocardial ischemia & Rat & $\begin{array}{l}\text { Increased myocardial nitrotyrosine } \\
\text { formation, decreased myofibrillar creatine } \\
\text { kinase activity. }\end{array}$ & 878,987 \\
\hline Chronic myocardial ischemia & Mouse & $\begin{array}{l}\text { Increased myocardial iNOS expression, } \\
\text { plasma nitrate and nitrite concentrations, } \\
\text { and myocardial and plasma nitrotyrosine } \\
\text { levels in wild-type compared with iNOS(-/ } \\
\text {-) mice. Improved LV function in iNOS(-- } \\
- \text { ) compared with wild-type mice. }\end{array}$ & 383 \\
\hline Pacing & Dog & $\begin{array}{l}\text { Progressively increased cardiac } \\
\text { dysfunction, nitrotyrosine formation, and } \\
\text { cell death in myocytes. }\end{array}$ & 192 \\
\hline${ }^{2}$ & Human & $\begin{array}{l}\text { Increased myocardial nitrotyrosine } \\
\text { formation in patients with HIV-induced } \\
\text { dilated cardiomyopathy. }\end{array}$ & 201 \\
\hline Idiopathic dilated cardiomyopathy & Human & $\begin{array}{l}\text { Increased iNOS expression and/or activity } \\
\text { in heart failure. }\end{array}$ & $\begin{array}{l}389,529,1317 \\
1465\end{array}$ \\
\hline Idiopathic dilated cardiomyopathy & Human & $\begin{array}{l}\text { Increased iNOS protein expression that is } \\
\text { associated with nitrotyrosine formation. } \\
\text { Although iNOS-positive patients are } \\
\text { generally characterized by larger LV } \\
\text { volume and depressed function, the } \\
\text { preserved NO generation appears to be } \\
\text { associated with higher cardiac work due to } \\
\text { the preserved Frank-Starling relationship } \\
\text { in end-stage heart failure. }\end{array}$ & 1317 \\
\hline Idiopathic dilated cardiomyopathy & Human & $\begin{array}{l}\text { Increased nitration of SERCA2a in } \\
\text { idiopathic dilated cardiomyopathic (DCM) } \\
\text { hearts. Positive correlation between the } \\
\text { time to half-relaxation and the } \\
\text { nitrotyrosine/SERCA2a content in } \\
\text { myocytes. }\end{array}$ & 789 \\
\hline
\end{tabular}




\begin{tabular}{|c|c|c|c|}
\hline Disease/Trigger & Experimental Model & Main Findings & Reference Nos. \\
\hline Atrial fibrillation & Atherosclerosis, resten & $\begin{array}{l}\text { Increased nitrotyrosine formation, which } \\
\text { correlates with myofibrillar creatine kinase } \\
\text { inhibition. } \\
\text { yperhomocysteinemia }\end{array}$ & 881 \\
\hline Hypercholesterolemia, hyperlipidemia & Rabbit, mouse & $\begin{array}{l}\text { The cholesterol-enriched diet increased } \\
\text { vascular superoxide and NT formation, } \\
\text { serum NT levels and induced endothelial } \\
\text { dysfunction. Cicletanine, estradiol and } \\
\text { fasting improved endothelial dysfunction, } \\
\text { decreased the atherosclerosis progression } \\
\text { and reduced serum or vascular NT level// } \\
\text { staining. }\end{array}$ & $653,1250,1411$ \\
\hline Hypercholesterolemia & Human & $\begin{array}{l}\text { Increased resting levels of ROS and } \\
\text { peroxynitrite in leukocytes from patients } \\
\text { with untreated hypercholesterolemia. }\end{array}$ & 343 \\
\hline Atherosclerosis & Human & $\begin{array}{l}\text { Increased 3-NT presence and iNOS } \\
\text { expression in human atherosclerotic tissue } \\
\text { that correlates with plaque instability in } \\
\text { patients. }\end{array}$ & $\begin{array}{l}49,80,164,240 \\
313,363,584,742, \\
802,1019,1211\end{array}$ \\
\hline $\begin{array}{l}\text { Vascular ballon injury (a model of } \\
\text { restenosis) }\end{array}$ & Rat, rabbit & $\begin{array}{l}\text { Increased 3-NT immunoreactivity, iNOS } \\
\text { overexpression, and PARP activation in } \\
\text { media and neointima following balloon } \\
\text { injury. }\end{array}$ & $\begin{array}{l}37,82,618,667 \\
747,921\end{array}$ \\
\hline Vascular stent implantation & Human & $\begin{array}{l}\text { Increased 3-NT/tyrosine ratio in the serum } \\
\text { of patients following stent implantation that } \\
\text { appeared to be an independent predictor of } \\
\text { angiographic late lumen loss. }\end{array}$ & 602 \\
\hline Hyperhomocysteinemia & Rat, mouse & $\begin{array}{l}\text { Endothelial dysfunction, impaired flow- } \\
\text { induced vasodilation, enhanced superoxide } \\
\text { and peroxynitrite production and NAD(P) } \\
\text { H oxidase expression in the vasculature, } \\
\text { enhanced myocardial NT formation, and } \\
\text { MMP activation. }\end{array}$ & $\begin{array}{l}42,70,339,579 \\
635,912,1181 \\
1303,1431,1435\end{array}$ \\
\hline Aging & Rat & $\begin{array}{l}\text { Cardiovascular dysfunction, increased } \\
\text { superoxide and peroxynitrite formation, } \\
\text { increased expression of NAD(P)H } \\
\text { oxidases, iNOS, and PARP activation in } \\
\text { aging vasculature and myocardium. } \\
\text { Enhanced age-dependent nitration of } \\
\text { various mitochondrial proteins (e.g., } \\
\text { aconitase, creatine kinase, voltage- } \\
\text { dependent anion channel, ATP synthase), } \\
\text { sarcoplasmic reticular Ca }{ }^{2+} \text {-ATPases, and } \\
\text { other proteins involved in blood } \\
\text { coagulation, lipid transport, blood pressure } \\
\text { regulation, and protease inhibition. }\end{array}$ & $\begin{array}{l}7,249,409,654 \\
676,692,991,992 \\
1131,1213,1311 \\
1329,1330,1397\end{array}$ \\
\hline \multicolumn{4}{|c|}{ Hypertension } \\
\hline Aortic banding & Mouse, rat & $\begin{array}{l}\text { Increased superoxide formation, gene } \\
\text { expression of several subunits of NAD(P) } \\
\text { H oxidases, NOS, and NT in the aorta } \\
\text { segment above aortic coarctation } \\
\text { (hypertensive zone) and in hearts of aortic- } \\
\text { banded rats and mice compared with } \\
\text { normotensive controls. }\end{array}$ & $65,125,724,1324$ \\
\hline $\begin{array}{l}\text { SHR, M-SHRSP, SHR/NDmcr-cp } \\
\text { (SHR/cp) }\end{array}$ & Rat & $\begin{array}{l}\text { Increased peroxynitrite formation or } \\
\text { protein nitration in the serum, vasculature, } \\
\text { and kidneys of SHR, malignant stroke- } \\
\text { prone spontaneously hypertensive (M- } \\
\text { SHRSP) and SHR/NDmcr-cp (SHR/cp) } \\
\text { rats (genetic model of the metabolic } \\
\text { syndrome). Antioxidant rich diet, M } 40403 \\
\text { (superoxide dismutase mimetic), } \\
\text { chlorogenic acid(polyphenol), ramipril } \\
\text { (angiotensin-converting enzyme inhibitor), } \\
\text { carvediiol (adrenoceptor blocker with } \\
\text { antioxidant properties) and } \\
\text { tetrahydrobiopterin (cofactor of eNOS) } \\
\text { attenuated hypertension and NT formation } \\
\text { in vasculature, kidneys, and improved } \\
\text { compromised vascular function and end- } \\
\text { organ damage. }\end{array}$ & $\begin{array}{l}269,565,643,776, \\
815,1080,1217 \\
1252,1402\end{array}$ \\
\hline Chronic ANG I infusion & Rat, mouse & $\begin{array}{l}\text { Increased peroxynitrite formation and } \\
\text { PARP activation in aorta, heart, and } \\
\text { kidneys of rats and mice following chronic }\end{array}$ & $\begin{array}{l}492,656,880, \\
1159,1236,1342 \\
1349\end{array}$ \\
\hline
\end{tabular}




\begin{tabular}{|c|c|c|c|}
\hline Disease/Trigger & Experimental Model & Main Findings & Reference Nos. \\
\hline & & $\begin{array}{l}\text { infusion and in endothelial cells in vitro. } \\
\text { The protein nitration correlated with the } \\
\text { extent of endothelial dysfunction observed } \\
\text { and both were attenuated by } \\
\text { supplementation with NOS cofactor } \\
\text { tetrahydrobiopterin. }\end{array}$ & \\
\hline Obesity/diet & Rat & $\begin{array}{l}\text { Long-term high-fat (primarily saturated) } \\
\text { diet induced hypertension associated with } \\
\text { increased accumulation of NT in aorta, } \\
\text { heart, kidney, and liver. Conversion to low- } \\
\text { fat diet normalized blood pressure and } \\
\text { tissue NT content. }\end{array}$ & 327,1077 \\
\hline Lead & Rat & $\begin{array}{l}\text { Lead-induced hypertension was associated } \\
\text { with increased ROS- mediated inactivation } \\
\text { of nitric oxide with sequential increase in } \\
\text { 3- NT abundance in plasma, heart, kidney, } \\
\text { and brain. Administration of high dose of } \\
\text { vitamin E ameliorated hypertension and } \\
\text { normalized tissue NT content without } \\
\text { altering tissue lead content. }\end{array}$ & 325,1323 \\
\hline Renovascular clamping/renal failure & Rat & $\begin{array}{l}\text { Increased NT formation in plasma, aorta, } \\
\text { heart, and kidney of rats with renal } \\
\text { hypertension [ } 1 \mathrm{KIC} \text { or } 2 \mathrm{KIC} \text { (one-kidney } \\
\text { or two-kidney one-clip) and } 5 / 6 \\
\text { nephrectomy]. This and hypertension were } \\
\text { attenuated by angiotensin type I receptor } \\
\text { inhibitor losartan, tempol, and high dose of } \\
\text { vitamin E. }\end{array}$ & $119,327,1325$ \\
\hline Mineralocorticoid (aldosterone) & Rat & $\begin{array}{l}\text { Chronic aldosterone/salt treatment induced } \\
\text { time-dependent sustained activation of } \\
\text { NADPH oxidase with 3-NT generation and } \\
\text { nuclear factor } \kappa \text { B activation in endothelial } \\
\text { and inflammatory cells, which was } \\
\text { attenuated by an aldosterone receptor } \\
\text { antagonist spironolactone. }\end{array}$ & 1214 \\
\hline Cyclosporin & Human & $\begin{array}{l}\text { In kidney-transplanted patients with } \\
\text { cyclosporin-induced hypertension, } \\
\text { carvediol increased plasma antioxidant } \\
\text { power and reduced 3-NT and TGF- } \beta \\
\text { mRNA levels. }\end{array}$ & 170 \\
\hline Hypertension, diabetes & Human cardiac myocytes & $\begin{array}{l}\text { Increased apoptosis, necrosis, angiotensin } \\
\text { II, and NT formation in myocytes. }\end{array}$ & 413 \\
\hline
\end{tabular}

NT/3-NT, nitrotyrosine/3-nitrotyrosine; iNOS, inducible nitric oxide synthase; MMP, matrix metalloproteinase; LV, left ventricle/ventricular; PARP, poly(ADP-ribose) polymerase; HIV, human immunodeficiency virus; ANG II, angiotensin II; ROS, reactive oxygen species; SHR, spontaneous hypertensive rat. 
TABLE 5

Evidence implicating endogenous peroxynitrite formation and/or protein nitration in diabetes and diabetic complications

\begin{tabular}{|c|c|c|c|}
\hline Disease Model, Trigger & Tissue, Function Investigated & Main Findings & Reference Nos. \\
\hline \multicolumn{4}{|c|}{ General } \\
\hline T2DM & Human plasma & $\begin{array}{l}\text { Cardiopulmonary bypass induced greater } \\
\text { oxidative and nitrosative stress in diabetic } \\
\text { patients. }\end{array}$ & 850 \\
\hline T2DM & Human plasma & $\begin{array}{l}\text { Increased plasma nitrite/nitrate and NT levels } \\
\text { in diabetic patients. Postprandial } \\
\text { hypertriglyceridemia and hyperglycemia } \\
\text { induces endothelial dysfunction in diabetic } \\
\text { patients and increased plasma NT levels. } \\
\text { Plasma NT levels in diabetic patients } \\
\text { correlate with postprandial hyperglycemia. }\end{array}$ & $36,186,188,190$ \\
\hline T1DM & Human plasma & $\begin{array}{l}\text { Increased plasma nitrite, nitrate, and NT, } \\
\text { which correlate with the insulin requirements } \\
\text { of the diabetic patients. }\end{array}$ & 553,555 \\
\hline T1DM, T2DM & Human platelets & $\begin{array}{l}\text { Increased iNOS-derived peroxynitrite } \\
\text { formation in diabetic platelets. }\end{array}$ & 1265 \\
\hline \multicolumn{4}{|c|}{ Primary diabetes } \\
\hline $\begin{array}{l}\text { NOD mice, STZ- induced } \\
\text { diabetes }\end{array}$ & Mouse pancreatic islet $\beta$-cells & $\begin{array}{l}\text { Increased NT formation in pancreatic islet } \beta \text { - } \\
\text { cells. Inhibitors of iNOS and/or scavengers or } \\
\text { peroxynitrite prevent development of } \\
\text { diabetes and reduce NT staining in the islets } \\
\text { in various experimental models. }\end{array}$ & $\begin{array}{l}822,1209,1210 \\
1234\end{array}$ \\
\hline \multicolumn{4}{|c|}{ Diabetic vascular dysfunction } \\
\hline High glucose & Human aortic endothelial cells & $\begin{array}{l}\text { Increased peroxynitrite formation, tyrosine } \\
\text { nitration, and inhibition of prostacyclin } \\
\text { synthase. }\end{array}$ & 1473 \\
\hline $\begin{array}{l}\text { Stable or intermittent high } \\
\text { glucose }\end{array}$ & $\begin{array}{l}\text { Human umbilical vein endothelial } \\
\text { cells }\end{array}$ & $\begin{array}{l}\text { Stable or intermittent high glucose stimulated } \\
\text { NT formation through PKC-dependent } \\
\text { activation of NAD }(\mathrm{P}) \mathrm{H} \text { oxidase. }\end{array}$ & 1049 \\
\hline High glucose & Human aortic endothelial cells & $\begin{array}{l}\text { Glucose-induced activation of PKC resulted } \\
\text { in peroxynitrite formation and nitration of } \\
\text { prostacyclin synthase. }\end{array}$ & 236 \\
\hline High glucose & Bovine endothelial cells & $\begin{array}{l}\text { HG induced increased lipid peroxidation, } \\
\text { increased superoxide and peroxynitrite } \\
\text { formation, and PKC activity. }\end{array}$ & 1040 \\
\hline STZ-induced diabetes & $\begin{array}{l}\text { Mouse aorta, vascular and cardiac } \\
\text { function pancreatic islet } \beta \text {-cells }\end{array}$ & $\begin{array}{l}\text { Increased eNOS expression, NT formation, } \\
\text { and PARP activation in endothelium and } \\
\text { vascular smooth muscle. A peroxynitrite } \\
\text { decomposition catalyst improved vascular } \\
\text { and cardiac function and protected against } \\
\text { diabetes. }\end{array}$ & 1186,1234 \\
\hline STZ-induced diabetes & Rat aorta, liver, kidney & $\begin{array}{l}\text { Increased free radical and NO concentrations } \\
\text { in the liver, kidney, and aorta; increased } \\
\text { peroxynitrite formation in aorta. }\end{array}$ & 1198 \\
\hline Zucker diabetic rats & Rat aorta, vascular function & $\begin{array}{l}\text { Age-dependent increase of NT formation in } \\
\text { the vasculature and development of } \\
\text { endothelial dysfunction, which is attenuated } \\
\text { by a peroxynitrite scavenger, ebselen. }\end{array}$ & 138 \\
\hline LDL from T1DM patients & Human aortic endothelial cells & $\begin{array}{l}\text { Incubation of human aortic endothelial cells } \\
\text { with LDL from T1DM patients increased } \\
\mathrm{Na}^{+} \mathrm{K}^{+} \text {-ATPase and } \mathrm{Ca}^{2+} \text {-ATPase activities, } \\
\text { NOS activity, and peroxynitrite production. }\end{array}$ & 1050 \\
\hline T2DM and prediabetes & Human skin microvasculature & $\begin{array}{l}\text { Increased NT formation and PARP activation } \\
\text { in endothelial cells of diabetic and prediabetic } \\
\text { patients. }\end{array}$ & 1242 \\
\hline T1DM, preeclampsia & Human placental vasculature & $\begin{array}{l}\text { Increased NT formation in vascular } \\
\text { endothelium and villous stroma, attenuated } \\
\text { vasoconstrictor and vasodilatory responses in } \\
\text { diabetes and preeclampsia. } \\
\text { myopathy }\end{array}$ & 711,807 \\
\hline \multicolumn{4}{|c|}{ Diabetic cardiomyopathy } \\
\hline Alloxan-induced diabetes & Mouse heart mitochondria & Tyrosine nitration of mitochondrial proteins. & 1298 \\
\hline STZ-induced diabetes & Mouse cardiac myocytes & $\begin{array}{l}\text { Increased apoptosis, } \mathrm{H}_{2} \mathrm{O}_{2} \text {, superoxide, } \\
\text { angiotensin II and NT formation in myocytes, } \\
\text { which is decreased by IGF-I overexpression. }\end{array}$ & 646 \\
\hline STZ-induced diabetes & Rat heart mitochondria & $\begin{array}{l}\text { Increased nitration and inactivation of } \\
\text { succinyl-CoA:3-oxoacid CoA-transferase } \\
\text { (SCOT). }\end{array}$ & 1299 \\
\hline High glucose & Rat heart & $\begin{array}{l}\text { Perfusion of isolated hearts with high glucose } \\
\text { increased superoxide generation, NO, NT } \\
\text { formation, and iNOS expression. }\end{array}$ & 189 \\
\hline
\end{tabular}




\begin{tabular}{|c|c|c|c|}
\hline Disease Model, Trigger & Tissue, Function Investigated & Main Findings & Reference Nos. \\
\hline High glucose & Rat heart & $\begin{array}{l}\text { M40403 (SOD) mimetic decreases Q-T } \\
\text { interval prolongation, coronary perfusion } \\
\text { pressure and lipid peroxidation, NT } \\
\text { formation, and PARP activation. }\end{array}$ & 316 \\
\hline \multicolumn{4}{|c|}{$\begin{array}{l}\text { and NT formation in myocytes. } \\
\text { Diabetic nephropathy }\end{array}$} \\
\hline STZ-induced diabetes & Mouse kidney & $\begin{array}{l}\text { Increased renal NT and advanced glycation } \\
\text { end product formation, which is attenuated by } \\
\text { ramipril or aminoguanidine. }\end{array}$ & 402 \\
\hline STZ-induced diabetes & Rat kidney & $\begin{array}{l}\text { Increased superoxide and nitrotyrosine } \\
\text { formation in renal cortex. }\end{array}$ & 615 \\
\hline STZ-induced diabetes & Rat kidney & $\begin{array}{l}\text { Increased renal expression of } \mathrm{p} 47 \mathrm{phox} \text {, } \\
\text { hydrogen peroxide production, and NT } \\
\text { formation. }\end{array}$ & 974 \\
\hline Diabetic nephropathy & Human kidney & $\begin{array}{l}\text { Increased staining NT formation in tubuli of } \\
\text { diabetic patients. }\end{array}$ & 1277 \\
\hline High glucose & Retinal endothelial cells & $\begin{array}{l}\text { lopathy } \\
\text { High glucose induced increased NT } \\
\text { formation in retinal endothelial cells, which } \\
\text { was blocked by superoxide or peroxynitrite } \\
\text { scavengers, NOS, or aldose reductase } \\
\text { inhibitors. }\end{array}$ & 352 \\
\hline $\begin{array}{l}\text { BBZ/Wor rat model of } \\
\text { NIDDM }\end{array}$ & Rat retina & $\begin{array}{l}\text { Increased iNOS and nitrotyrosine } \\
\text { immunoreactivity in diabetic retinas. }\end{array}$ & 351 \\
\hline STZ-induced diabetes & Rat retina & $\begin{array}{l}\text { Increased NT, which is attenuated by } \\
\text { aminoguanidine or by an aldose reductase } \\
\text { inhibitor fidarestat. }\end{array}$ & 333,963 \\
\hline STZ-induced diabetes & Rat retina & $\begin{array}{l}\text { Increased retinal lipid peroxidation and NT } \\
\text { formation, which was only slightly attenuated } \\
\text { by reinstitution of good glycemic control. }\end{array}$ & 712 \\
\hline STZ-induced diabetes & Rat retina & $\begin{array}{l}\text { Increased tyrosine nitration and expression of } \\
\text { vascular endothelial growth factor contribute } \\
\text { to the breakdown of the blood-retina barrier } \\
\text { in diabetes. } \\
\text { opathy }\end{array}$ & 354 \\
\hline STZ-induced diabetes & $\begin{array}{l}\text { Rat peripheral nerves, epineurial } \\
\text { arterioles, endoneurial blood flow }\end{array}$ & $\begin{array}{l}\text { Antioxidants reduced the production of } \\
\text { superoxide and peroxynitrite in epineurial } \\
\text { arterioles and improved endoneural blood } \\
\text { flow. }\end{array}$ & $230-232$ \\
\hline STZ-induced diabetes & Rat peripheral sensory neurons & $\begin{array}{l}\text { Rise in cytoplasmic labeling of nitrotyrosine, } \\
\text { PARP activation. }\end{array}$ & 209 \\
\hline $\begin{array}{l}\text { STZ-induced diabetes, NOD } \\
\text { mice }\end{array}$ & $\begin{array}{l}\text { Mouse sciatic motor and hindlimb } \\
\text { digital sensory nerve }\end{array}$ & $\begin{array}{l}\text { A peroxynitrite scavenger FP- } 15 \text { normalizes } \\
\text { diabetes-associated decreased sciatic motor } \\
\text { nerve and digital sensory nerve conduction } \\
\text { velocity, and ameliorated the NT formation } \\
\text { and poly(ADP-ribose) accumulation in } \\
\text { diabetic nerves. }\end{array}$ & 961 \\
\hline T1DM & $\begin{array}{l}\text { Human peripheral motor nerve } \\
\text { function } \\
\qquad \text { Diabe }\end{array}$ & $\begin{array}{l}\text { Decreased motor nerve function in diabetic } \\
\text { patients correlates with increased nitrosative } \\
\text { stress. } \\
\text { opathy }\end{array}$ & 554 \\
\hline STZ-induced diabetes & Rat bladder & $\begin{array}{l}\text { Increased proteasomal activation and NT } \\
\text { formation during diabetic cystopathy. }\end{array}$ & 1034 \\
\hline
\end{tabular}

T2DM, type 2 diabetes mellitus; T1DM, type 1 diabetes mellitus; NT/3-NT, nitrotyrosine/3-nitrotyrosine; iNOS, inducible nitric oxide synthase; NOD, non-obese diabetic; STZ, streptozotocin; SOD, superoxide dismutase; PARP, poly(ADP-ribose) polymerase. 